\title{
BROADBAND PHOTON ABSORPTION IN SELF-ASSEMBLED NETWORKS OF MULTIPHASE OXIDES OF TITANIUM
}

\author{
by \\ Pooja Thakur \\ Master of Engineering, University of Toronto (2011) \\ Bachelor of Engineering, Osmania University (2009)
}

\author{
A dissertation \\ presented to Ryerson University \\ in partial fulfillment of the \\ requirements for the degree of \\ Doctor of Philosophy \\ in the Program of \\ Aerospace Engineering
}

Toronto, Ontario, Canada, 2017

(C) Pooja Thakur, 2017 


\section{Author's Declaration}

I hereby declare that I am the sole author of this dissertation. This is a true copy of the dissertation, including any required final revisions, as accepted by my examiners.

I authorize Ryerson University to lend this dissertation to other institutions or individuals for the purpose of scholarly research.

I further authorize Ryerson University to reproduce this dissertation by photocopying or by other means, in total or in part, at the request of other institutions or individuals for the purpose of scholarly research.

I understand that my dissertation may be made electronically available to the public.

Pooja Thakur 


\section{Abstract}

\section{BROADBAND PHOTON ABSORPTION IN SELF-ASSEMBLED NETWORKS OF MULTIPHASE OXIDES OF TITANIUM}

\section{Pooja Thakur}

Doctor of Philosophy, Aerospace Engineering, Ryerson University, Toronto (2017)

Titanium -dioxide $\left(\mathrm{TiO}_{2}\right)$ has garnered immense interest as a potential photon absorber after the discovery of its photocatalytic properties. However, its absorption is limited to the ultraviolet region of the solar spectrum. Despite numerous efforts being made, the challenge to extend its absorption capability to the entire visible and near infrared region (vis-NIR) still exists, which together constitute about $90 \%$ of the solar spectrum.

In this dissertation, a multiphase nano $\mathrm{TiO}_{\mathrm{x}}$ network, rich in defects and oxygen vacancies, has been presented which can absorb photons over a broader range of the solar spectrum.

Experimental studies were initially conducted to phase functionalise titanium towards enhanced photon absorption via a single step, ultrashort laser pulse material interaction process. This phase functionalised titanium, characterised to be uniquely composed of multiple oxide phases of titanium, can effectively absorb photons in the vis-NIR region

Using the above study as a template, a complex three-dimensional self-assembled nano network composed of similar multiphase titanium oxides, was then synthesised. Free of any external dopants, it exhibits a remarkable absorption of photons ranging from 300-1000 nm. To further improve the absorptive properties of this 'multiphase nano $\mathrm{TiO}_{\mathrm{x}}$ network', particularly in the lower 
visible range, the phenomenon of Surface Plasmon Resonance was utilised via its hybridisation with gold and gold/palladium alloy. This successfully resulted in further optimisation of its absorption.

The final study of the multiphase nano $\mathrm{TiO}_{\mathrm{x}}$ was done to understand the fundamental physics behind its broadened photon absorptive behaviour. The condition of synthesis was varied by introducing various contrasting plasma environments. Pronounced disorders and oxygen defects of varying degrees within the crystalline structure were observed. The enhanced and broadened absorption spectrum achieved was attributed to such defects and disorders.

The research done in this thesis demonstrates a unique nanomaterial based on multiple oxides phases of titanium that is capable of absorbing photons both in the visible and NIR regions. The contribution made towards the synthesis, investigation and subsequent manipulation of the selfassembled multiphase nano $\mathrm{TiO}_{\mathrm{x}}$ network can thus be exploited in various photon harvesting applications like photovoltaics and photo catalysis, where such a broadband photon absorption is desirable. 


\section{Acknowledgements}

I would like to express my gratitude to my supervisors, Dr. Bo Tan and Dr. Krishnan Venkatakrishnan, for their constant support and encouragement. Their insightful guidance and faith in my abilities helped me towards the successful completion of this dissertation.

I would also like to humbly thank all the committee members for providing valuable inputs to improve this dissertation. I am grateful to Dr. Jeffrey Yokota and Leah Rogan, for patiently answering all my queries and providing vital information about Graduate Studies.

I would like to thank my friends and lab mates at Ryerson University, for providing a dynamic work environment. I am grateful for the support given by Jeffery Powell, Sivaprasad Vijaykumar, and Meysam Keshavarz. A special thanks to Priyatha Premnath for always providing joy and solace during my tough times. My sincere gratitude to Pengqian Xu (Kyle), for his hard work on statistical analysis and graphical illustrations for my research. I am grateful to Sahri Woods Baum for helping me work towards my self-development.

I would like to thank my entire family. Most importantly, my mother, Surekha Sanjay, for her unconditional love and patience. She has always played an integral role towards my education. My partner for life, Saurav Mohapatra. Words fail to express the amount of love and gratitude I hold for him, for his unconditional support and time. I thank him for holding me up when I was at my most vulnerable.

Finally, I would like to thank my wonderful papa, Late Thakur Sanjay Prasad. He was instrumental in always encouraging me to think big and pursue my dreams. He inspired me to be independent and follow my heart with a clear conscience. I miss him dearly and lovingly dedicate this thesis to him. 
To Papa.

I am what I am because of you.

I miss you... 


\section{Table of Contents}

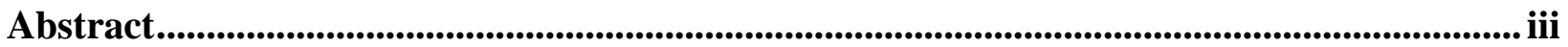

List of Tables ........................................................................................................................................

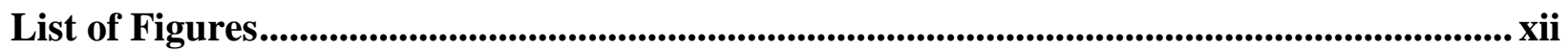

Chapter 1 Introduction................................................................................................................................... 1

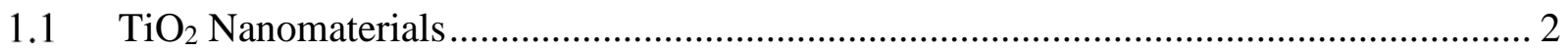

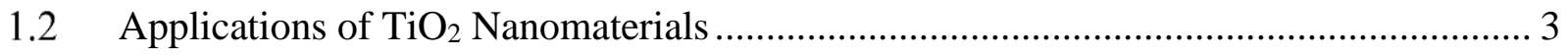

1.3 Efforts to Enhance the Optical Absorption of $\mathrm{TiO}_{2}$ Nanomaterials .................................. 4

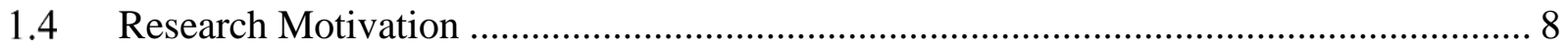

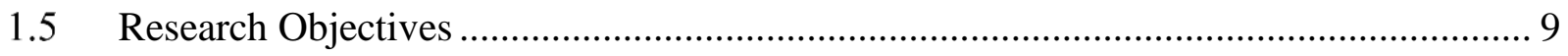

1.6 Dissertation Organisation................................................................................... 10

Chapter 2 Laser Synthesis Mechanism.............................................................................................. 12

2.1 Physics of Ultrafast Laser-Material Interaction ........................................................ 12

2.2 Surface Temperature of Target Material .................................................................. 14

2.3 Nanostructure Formation and Growth..................................................................... 16

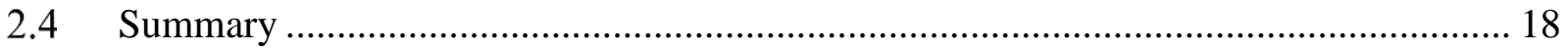

Chapter 3 Experimental Setup and Procedure .................................................................................. 19

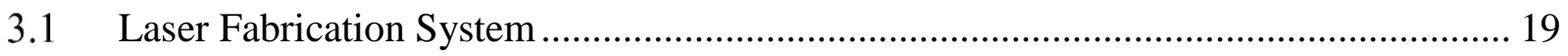




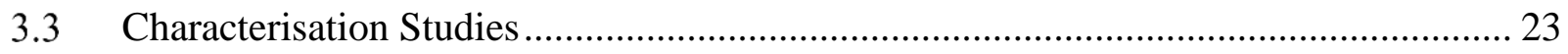

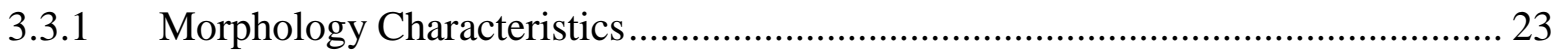

3.3.2 Chemical and phase characterisations ........................................................ 24

3.3.3 Absorption, Emission and Raman Spectroscopic Characterisation ...................... 26

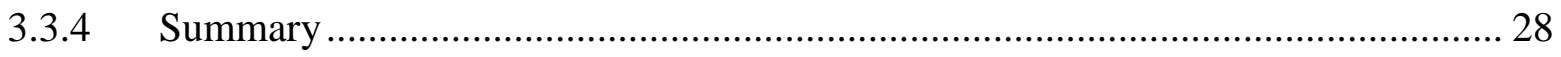

Chapter 4 Multiphase Functionalization of Titanium........................................................ 29

4.1 Fabrication of Phase Functionalized Titanium..................................................... 30

4.2 Material Characterization Studies ..................................................................... 31

4.3 Photon Absorption Under Various Synthesis Conditions ......................................... 38

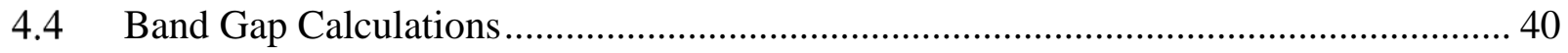

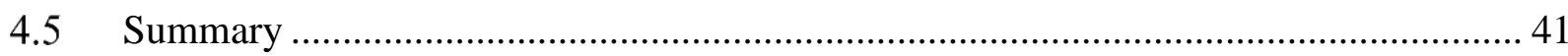

Chapter 5 Synthesis of Multiphase Nano TiO ${ }_{x}$ Networks ...................................................... 43

5.1 Self-Assembly of $\mathrm{Nano}^{\mathrm{TiO}}$ x Network ........................................................... 43

5.2 Surface and Material Phase Characterization.................................................... 45

5.3 Photon Absorption and Raman Enhancement.................................................... 53

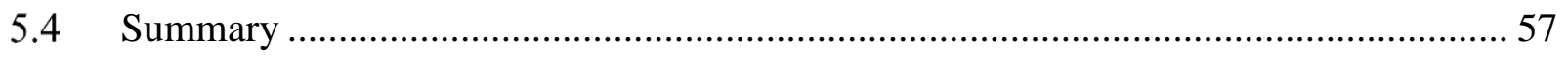

Chapter 6 Plasmon Hybridization of Multiphase Nano TiOx Networks ................................. 58

6.1 Induction of Localized Surface Plasmon Behaviour Using Au and Au/Pd Alloy ........ 60

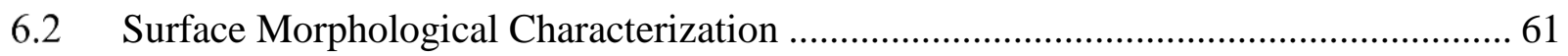


6.3 Enhanced Photon Absorptive Behaviour

6.4 Changes in Absorbance due to Variations in Morphology and Phase Composition ..... 73

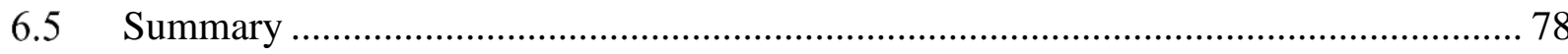

Chapter 7 Broadband Absorption Spectrum with Disordered Multiphase Nano TiO

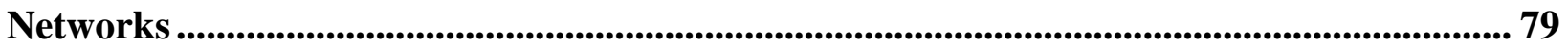

7.1 Disordered Crystal Formation by Alteration of Plume Kinetics .............................. 80

7.2 Surface and Material Characterization of Disordered Multiphase Nano $\mathrm{TiO}_{\mathrm{x}} \ldots \ldots \ldots \ldots . . . .83$

7.3 Analysis of Defect Types and Densities ...................................................... 94

7.4 Absorption Spectrum and Band Gap Calculation ............................................... 100

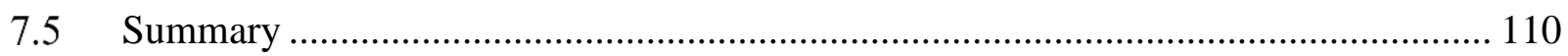

Chapter 8 Proposed Applications for Multiphase Nano TiOx ............................................. 111

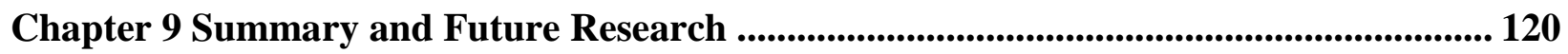

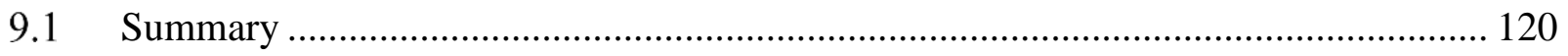

9.2 Recommended Future Research................................................................ 122

Appendices ............................................................................................................................................................. 124

A. Surface Temperature Calculation and Analysis .............................................. 124

B. Calculation of Enhancement Factor ................................................................ 130

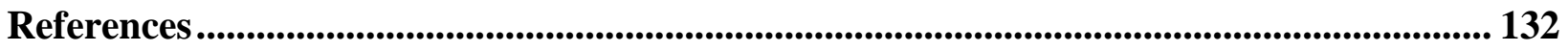

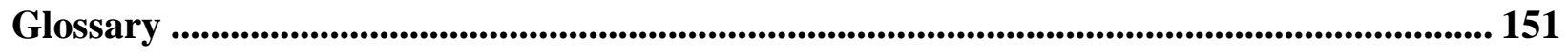

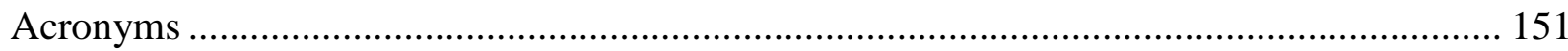


Units

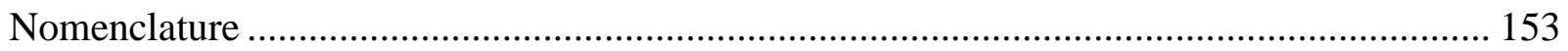

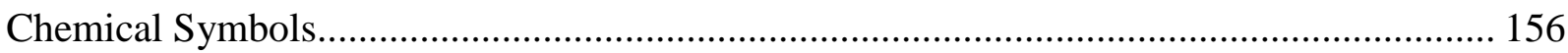




\section{List of Tables}

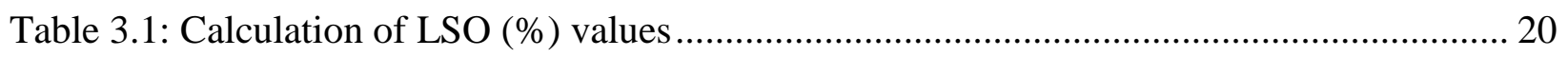

Table 4.1: Band gap values, Eg (using Tauc's equation), of the functionalised Ti generated at

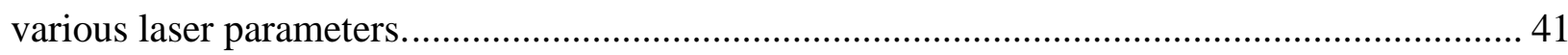




\section{List of Figures}

Figure 1.1: Illustration of the modification paths for $\mathrm{TiO}_{2}$ nanomaterials [22] ....................... 4

Figure 1.2: Absorption/Reflectance spectrum $\mathrm{TiO}_{2}$ nanomaterials doped with (A) Nitrogen (N), Tin(Sn) [33], (B) Nitrogen [27], (C) Cerium (Ce) [34], (D) Silver (Ag) [35], (E) Ruthenium (Ru)

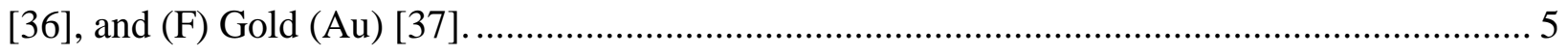

Figure 1.3: Plasmon absorption spectrum of $\mathrm{TiO}_{2}$ loaded with (A) Gold (Au) [50], (B) Silver (Ag) [51], and (C) Absorption spectrum of $\mathrm{TiO}_{2}$ doped with Carbon and Fluorine and loaded with a

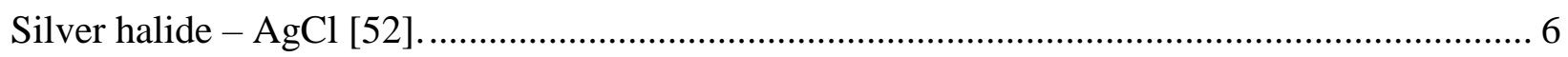

Figure 1.4 Absorption spectrum of various 'self doped' $\mathrm{TiO}_{2}$ nanomaterials (A) stable blue $\mathrm{TiO}_{2-\mathrm{x}}$

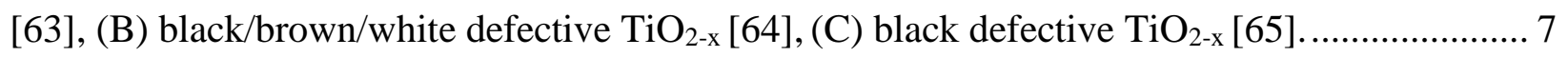

Figure 2.1Temperature of the laser spot area on the target surface upon irradiation with a series of

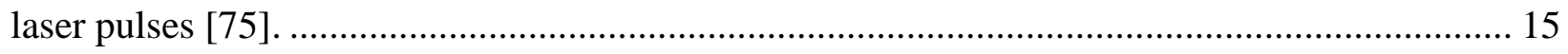

Figure 3.1: Isometric view (top) \& top view (bottom) of the experiment setup of the laser....... 21

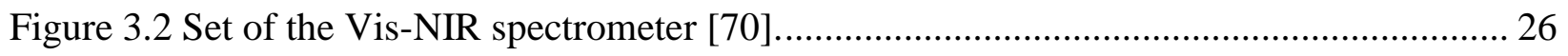

Figure 4.1 (A), (B) EDX line scan showing the variation of Oxygen and Titanium content along the base Titanium substrate and the laser functionalised zone, and (C) O/Ti ratio plot. 31

Figure 4.2: wt. $\%$ distribution of each of the three identified Titanium Oxides as obtained by the Rietveld fitting and the corresponding XRD patterns on the right, obtained at various laser parameters. 
Figure 4.3: (A) Ti 2p XPS spectrum, and (B) Raman spectrum of the laser functionalised zone when synthesised at different laser conditions. 34

Figure 4.4: HRTEM image of (A) rutile $\mathrm{TiO}_{2}$ particle showing different planes in area 1 and 2, (B) hexagonal $\mathrm{Ti}_{3} \mathrm{O}$, with the FFT and magnified image given in the inset, (C) d-spacing values of different planes of $\mathrm{Ti}_{3} \mathrm{O}$ as measured from figure (B) and standard (PDF2 source) values, (D) fccstructured ( $\left.\mathrm{TiO}_{716}\right)_{3.76}$ (area 1 and 3) and $\mathrm{Ti}_{3} \mathrm{O}$ (area 2), with the FFT image given in the inset, and (E) Measured and standard (PDF2 source) d-spacing values of fringes present in different marked areas of figure and the corresponding identified Oxide of Titanium. 36

Figure 4.5: Neff vs. Laser fluence plot with the corresponding SEM images of the laser functionalised zone at extreme condition of laser fluence. 37

Figure 4.6: Absorbance measured at different wavelengths for the three pulse width values of $214 \mathrm{fs}$ (red), $714 \mathrm{fs}$ (green), $1428 \mathrm{fs}$ (blue), at a constant laser fluence value of (A) $2.4614 \mathrm{~J} / \mathrm{cm}^{2}$, (B) $1.6409 \mathrm{~J} / \mathrm{cm}^{2}$, and (C) $0.8082 \mathrm{~J} / \mathrm{cm}^{2}$. 39

Figure 5.1: Multiphase Titanium Oxide nanomaterial formed by ultra-short laser synthesis mechanism in ambient air. 44

Figure 5.2: SEM images of the nanomaterial network generated at (A) $1.64 \mathrm{~J} / \mathrm{cm}^{2}$, (B) $2.46 \mathrm{~J} / \mathrm{cm}^{2}$, and (C) $5.14 \mathrm{~J} / \mathrm{cm}^{2}$ laser fluence values. 46

Figure 5.3: Size distribution of the nanospheres of the nano network synthesised at laser fluence and pulse width values of (A) $5.14 \mathrm{~J} / \mathrm{cm}^{2}, 214 \mathrm{fs}$, (B) $1.64 \mathrm{~J} / \mathrm{cm}^{2}, 214 \mathrm{fs}$, and (C) $5.14 \mathrm{~J} / \mathrm{cm}^{2}$, 1428 fs calculated using TEM micrographs. Inset shows a TEM micrograph of the nanostructures formed at the given laser condition. 47 
Figure 5.4: (A) Electron image of nanonetwork captured by HR-SEM and the corresponding EDX area scan showing its (B) Oxygen, and (C) Titanium elemental distribution. (D) EDX Line scan analysis of a single spherical nanostructure.

Figure 5.5: XPS spectrum showing a chemical shift for the nanomaterial synthesised at different lasing conditions. 50

Figure 5.6: XRD patterns obtained for the multiphase nanomaterial synthesised at a laser fluence of (A) $5.14 \mathrm{~J} / \mathrm{cm}^{2}$, and (B) $1.64 \mathrm{~J} / \mathrm{cm}^{2}$. 51

Figure 5.7: Pie charts showing the Rietveld fitted weight percentage values of all the identified phases for the multiphase nano $\mathrm{TiO}_{\mathrm{x}}$ network synthesised at a laser fluence and pulse width values of $\quad$ (A) $5.14 \mathrm{~J} / \mathrm{cm}^{2}, 214 \mathrm{fs}, \quad$ (B) $5.14 \mathrm{~J} / \mathrm{cm}^{2}, 1428 \mathrm{fs}, \quad$ (C) $1.64 \mathrm{~J} / \mathrm{cm}^{2}, 214 \quad$ fs, and $\quad$ (D) 1.64 $\mathrm{J} / \mathrm{cm}^{2}, 1428$ fs. 53

Figure 5.8: Absorption spectrum of the multiphase nano $\mathrm{TiO}_{\mathrm{x}}$ network synthesised at laser fluence of (a) $5.14 \mathrm{~J} / \mathrm{cm} 2$, (b) $2.46 \mathrm{~J} / \mathrm{cm} 2$ and (c) $1.64 \mathrm{~J} / \mathrm{cm} 2$ and different laser pulse widths. 54

Figure 5.9: Raman Spectra obtained at two laser beam wavelengths (532 $\mathrm{nm}$ and $785 \mathrm{~nm})$ for both the base Titanium substrate and the multiphase nano $\mathrm{TiO}_{\mathrm{x}}$ at laser fluence and pulse width values of (A) $5.14 \mathrm{~J} / \mathrm{cm}^{2}, 214 \mathrm{fs}$, (B) $5.14 \mathrm{~J} / \mathrm{cm}^{2}, 1428 \mathrm{fs}$, (C) $2.46 \mathrm{~J} / \mathrm{cm}^{2}, 214 \mathrm{fs}$, (D) $2.46 \mathrm{~J} / \mathrm{cm}^{2}, 1428 \mathrm{fs}$, (E) $1.64 \mathrm{~J} / \mathrm{cm}^{2}, 214 \mathrm{fs}(\mathrm{F}) 1.64 \mathrm{~J} / \mathrm{cm}^{2}, 1428 \mathrm{fs}$. The Raman spectra of the base Titanium substrate obtained at $532 \mathrm{~nm}$ and $785 \mathrm{~nm}$ laser beam wavelength is indicated in black and red colour respectively. The Raman spectra of the nanomaterial obtained at $532 \mathrm{~nm}$ and $785 \mathrm{~nm}$ laser beam wavelength is indicated in blue and olive green colour respectively. 56

Figure 6.1:(A) Graphical illustration of the fabrication mechanism of the core self-assembled multiphase nano $\mathrm{TiO}_{\mathrm{x}}$ followed by its plasmon hybridisation with (B) Au and (C) Au/Pd alloy. 61 
Figure 6.2: SEM images of the self-assembled 3D network of core Titanium Oxide multiphase nanomaterial plasmon hybridised with (A) Au, and (B) Au/palladium alloy..... 63

Figure 6.3: TEM images of the (A) Self-assembled core nano $\mathrm{TiO}_{\mathrm{x}}$ nanomaterial consisting of aggregated nanospheres which are subsequently plasmon hybridised with (B) Au and (C) Au/Pd alloy. 65

Figure 6.4: Raman spectra obtained at a laser beam wavelength of $532 \mathrm{~nm}$ for the multiphase nano $\mathrm{TiO}_{\mathrm{x}}$ before and after its plasmon hybridisation with (A) $\mathrm{Au}$, and (B) $\mathrm{Au} / \mathrm{Pd}$ alloy. The Raman spectra for the base Titanium substrate with and without the noble metal hybridisation has also been plotted. 66

Figure 6.5: Broadening and enhancement observed in the absorption spectrum of the plasmon hybridised multiphase nano $\mathrm{TiO}_{\mathrm{x}}$ with respect to titanium substrate. 67

Figure 6.6: Variation in the degree of coverage and hybridisation of the core multiphase nano $\mathrm{TiO}_{\mathrm{x}}$ by coalesced islands of $\mathrm{Au}$, at different atomic $\%$ concentrations of (A) $1.74 \%$, and (B) $3.56 \%$.

Figure 6.7: Variation in the degree of coverage and hybridisation of the core nano $\mathrm{TiO}_{\mathrm{x}}$ by $\mathrm{Au} / \mathrm{Pd}$ alloy, at different atomic \% concentrations of (A) $0.82 \% \mathrm{Au}, 0.30 \% \mathrm{Pd}$, and (B) $1.63 \% \mathrm{Au}$, $0.68 \% \mathrm{Pd}$ 72

Figure 6.8: Absorption spectrum of the core nano $\mathrm{TiO}_{\mathrm{x}}$ synthesised at $1.64 \mathrm{~J} / \mathrm{cm}^{2}$ laser fluence and 1428 fs pulse width and having the same phase composition but plasmon hybridised with different atomic \% concentrations of (A) Au, and (B) Au/palladium alloy. The various colours in the pie chart schematic, representing an individually plasmon hybridised nanosphere constituting the 
nanomaterial network, indicate the various Titanium Oxide phases: cyan - rutile, blue - anatase.

Figure 6.9: Absorption spectrum of the core nano $\mathrm{TiO}_{\mathrm{x}}$ synthesised at a laser fluence of $1.64 \mathrm{~J} / \mathrm{cm}^{2}$ and two pulse widths values (214 fs and $1428 \mathrm{fs}$ ), and plasmon hybridised with a constant atomic $\%$ concentrations of (A) $\mathrm{Au}(1.74 \% \mathrm{Au})$, and (B) Au/palladium alloy $(0.82 \% \mathrm{Au}$, $0.30 \% \mathrm{Pd})$. The various colours in the pie chart schematic, representing an individually plasmon hybridised nanosphere constituting the nanomaterial network, indicate the various Titanium Oxide phases: cyan - rutile, blue - anatase. 76

Figure 6.10: Absorption spectrum of the core self-assembled multiphase nano $\mathrm{TiO}_{\mathrm{x}}$ synthesised at a pulse width of $1428 \mathrm{fs}$ and two laser fluence values $\left(5.14 \mathrm{~J} / \mathrm{cm}^{2}\right.$ and $\left.1.64 \mathrm{~J} / \mathrm{cm}^{2}\right)$ and plasmon hybridised with a constant atomic \% concentrations of (A) Au (1.74\% Au), and (B) Au/palladium alloy $(0.82 \% \mathrm{Au}, 0.30 \% \mathrm{Pd})$. The various colours in the pie chart schematic, representing an individually and plasmon hybridised nanosphere constituting the nanomaterial network, indicate the various Titanium Oxide phases: cyan - rutile, blue - anatase, black $-\alpha \mathrm{Ti}$, red $-\mathrm{Ti}_{3} \mathrm{O}$, green$(\mathrm{TiO} .716) 3.76$. 77

Figure 7.1: Graphical representation of the synthesis of nano $\mathrm{TiO}_{\mathrm{x}}$ under (a) poor Oxygen, and (b) Oxygen rich conditions, with the variation in the expansion of the laser plume and the ionic content around it. 83

Figure 7.2: FE-SEM images of the 3D self assembled network of nano TiOx synthesised under (a) Oxygen rich, and (b) Oxygen poor conditions. Magnified images show the network being comprised of nanospheres self-assembled to form the structure. 84 
Figure 7.3: XPS spectra of the nano Titanium. (a) Ti 2p spectra, (b) O s1 spectra, and (c) Valence band (VB) spectra. Purple indicates the spectra of the nano $\mathrm{TiO}_{\mathrm{x}}$ synthesised under Oxygen poor conditions and a laser intensity of $7.2 \times 1014 \mathrm{~W} / \mathrm{cm}^{2}$. Green colour indicates the spectra of the nano $\mathrm{TiO}_{\mathrm{x}}$ synthesised under Oxygen rich conditions and a laser intensity of $1.4 \times 1014 \mathrm{~W} / \mathrm{cm}^{2}$. Inset in (b) shows the shift in binding energy and the slopes marked in (c) indicate the valance band values. 86

Figure 7.4: XRD pattern of the nano $\mathrm{TiO}_{\mathrm{x}}$ synthesised under Oxygen poor conditions and a laser intensity of (a) $1.4 \times 1014 \mathrm{~W} / \mathrm{cm}^{2}$ and (b) $7.2 \times 1014 \mathrm{~W} / \mathrm{cm}^{2}$, and under Oxygen rich conditions and a laser intensity of (c) $1.4 \times 1014 \mathrm{~W} / \mathrm{cm}^{2}$ and (d) $7.2 \times 1014 \mathrm{~W} / \mathrm{cm}^{2}$. Different coloured bars at the bottom of the plot represent the reference XRD peaks from PDF-2 and ICSD database corresponding to each of the phase constituents. A few of the areas of the XRD pattern where peak broadening and splitting was observed has been magnified and shown. 90

Figure 7.5: Size distribution of the individual nanospheres constituting the network of nano $\mathrm{TiO}_{\mathrm{x}}$ synthesised at different laser intensities and under Oxygen rich (a) $2.2 \times 1014 \mathrm{~W} / \mathrm{cm}^{2}$, (b) $4.6 \times 1014 \mathrm{~W} / \mathrm{cm}^{2}$ and (c) $7.2 \times 1014 \mathrm{~W} / \mathrm{cm}^{2}$, and Oxygen poor conditions (d) $1.1 \times 1014 \mathrm{~W} / \mathrm{cm}^{2}$, (e) $4.6 \times 1014 \mathrm{~W} / \mathrm{cm}^{2}$ and (f) $7.2 \times 1014 \mathrm{~W} / \mathrm{cm}^{2}$. Inset are the corresponding TEM images....... 93 Figure 7.6: Variation of mean nanosphere size with laser intensity, as seen in the distribution plots for both Oxygen rich and Oxygen poor conditions. Errors bars indicate the standard deviation. 94 Figure 7.7: HR- TEM image of the nano TiOx showing higher degree of crystallinity and defects in the nanospheres when synthesised under (a) poor Oxygen conditions than at (b) Oxygen rich conditions. 95 
Figure 7.8: HRTEM micrographs of nano TiOx showing single nanospheres (a)-(h) having numerous Oxygen vacancies and defects marked with white dashed circles. The calculated d-spacing is marked and FFT images are given as inset.

Figure 7.9: Raman spectra of HDD and LDD-nano $\mathrm{TiO}_{\mathrm{x}}$ formed under different laser intensities of (a) $7.4 \times 1013 \mathrm{~W} / \mathrm{cm}^{2}$, (b) $1.1 \times 1014 \mathrm{~W} / \mathrm{cm}^{2}$, (c) $1.4 \times 1014 \mathrm{~W} / \mathrm{cm}^{2}$, (d) $2.2 \times 1014 \mathrm{~W} / \mathrm{cm}^{2}$, (e) $4.6 \times 1014 \mathrm{~W} / \mathrm{cm}^{2}$, and (f) $7.2 \times 1014 \mathrm{~W} / \mathrm{cm}^{2}$ 99

Figure 7.10: Negative Raman shift with respect to the active modes of standard rutile peaks, as observed in the LDD and HDD-nano $\mathrm{TiO}_{\mathrm{x}}$ synthesised at different laser intensities. 100

Figure 7.11: Absorption Spectrum of HDD-nano $\mathrm{TiO}_{\mathrm{x}}$ (thick blue line) and $\mathrm{LDD}$-nano $\mathrm{TiO}_{\mathrm{x}}($ thin red line), synthesised at increasing laser intensities of (a) $7.4 \times 1013 \mathrm{~W} / \mathrm{cm}^{2}$, (b) $1.1 \times$ $1014 \mathrm{~W} / \mathrm{cm}^{2}$, (c) $1.4 \times 1014 \mathrm{~W} / \mathrm{cm}^{2}$, (d) $2.2 \times 1014 \mathrm{~W} / \mathrm{cm}^{2}$, (e) $4.6 \times 1014 \mathrm{~W} / \mathrm{cm}^{2}$, and (f) $7.2 \times 1014 \mathrm{~W} / \mathrm{cm}^{2}$. Given inset are the $F R h v 2$ versus $E$ plot, representing the kubelka-munk method to calcualte the corresponding band gap of the HDD-nano $\mathrm{TiO}_{\mathrm{x}}$. 103

Figure 7.12: Absorption spectrum of the HDD-nano $\mathrm{TiO}_{\mathrm{x}}$ with respect to titanium substrate, when synthesised at increasing laser intensity values. For better representation, the same spectrum is magnified and split into (a) UV range, (b) Visible range, and (c) NIR range. 104

Figure 7.13: The excitation/emission spectra of $\mathrm{HDD}$-nano $\mathrm{TiO}_{\mathrm{x}}$ at laser intensity of (a) $4.6 \times 1014 \mathrm{~W} / \mathrm{cm}^{2}$ and (b) $7.2 \times 1014 \mathrm{~W} / \mathrm{cm}^{2}$, and the corresponding dual Raman spectra is given in (c) and (d). The area of the spectra indicating possible up-conversion is magnified and given as inset. 108

Figure 7.14: The excitation/emission spectra of $\mathrm{LDD}$-nano $\mathrm{TiO}_{\mathrm{x}}$ at laser intensity of (a) $4.6 \times 1014 \mathrm{~W} / \mathrm{cm}^{2}$ and (b) $7.2 \times 1014 \mathrm{~W} / \mathrm{cm}^{2}$ 109 
Figure 8.1 Comparison of the absorption spectrum of the $\mathrm{TiO}_{2}$ with that of HDD-Nano $\mathrm{TiO}_{\mathrm{x}}$ and LDD-Nano $\mathrm{TiO}_{\mathrm{x}}$. The more bluish colour of the LDD-Nano $\mathrm{TiO}_{\mathrm{x}}$ when compared with the grey coloured HDD-Nano $\mathrm{TiO}_{\mathrm{x}}$ is clearly seen in the photographs provided. The solar irradiance spectrum [148] is shown in back drop for comparison. 113

Figure 8.2: Graphical illustration of coating HDD-nano TiOx with CV dye. 114

Figure 8.3: Raman spectra CV dye on HDD-nano TiOx synthesised at laser intensities of (a) 4.6e14 $\mathrm{W} / \mathrm{cm}^{2}$ and $7.2 \mathrm{e} 14 \mathrm{~W} / \mathrm{cm}^{2}$. The Raman spectra of just the HDD nano TiOx and only the bare Ti substrate has also been plotted for comparison. The characteristic peaks of the CV dye have been marked. Magnified plots have been given as inset for the Raman spectra of CV dye on HDDnano $\mathrm{TiO}_{\mathrm{x}}$ for clarity of peaks. 115

Figure 8.4: Variation of the contact angle of the liquid droplet deposited on HDD-nano $\mathrm{TiO}_{\mathrm{x}}$ when subjected to (a) UV irradiation (b) visible irradiation. Photographs on the right show the shape of the droplet on $\mathrm{HDD}$-nano $\mathrm{TiO}_{\mathrm{x}}$ at different exposure times during irradiation. 118 Figure 9.1: Avg. surface temp. calculated for the first twenty laser pulses at different fluence values: (A) $2.4614 \mathrm{~J} / \mathrm{cm}^{2}$, (B) $1.6409 \mathrm{~J} / \mathrm{cm}^{2}$, (C) $0.8208 \mathrm{~J} / \mathrm{cm}^{2}$, and (D) variation of average surface temperature vs. laser fluence. 125

Figure 9.2: Thermal diffusion away from the laser material interaction spot for laser fluence of $0.8208 \mathrm{~J} / \mathrm{cm} 2$ right after the laser beam is removed (A) $0.2 \mathrm{~ms}$ and a few time periods after the end of the interaction, (B) $1 \mathrm{~ms}$, (C) $2 \mathrm{~ms}$, (D) $3 \mathrm{~ms}$, (E) $4 \mathrm{~ms}$, (F) $5 \mathrm{~ms}$, (G) $6 \mathrm{~ms}$, (H) $7 \mathrm{~ms}$, and (I) $8 \mathrm{~ms}$ 127

Figure 9.3: Temperature $[\mathrm{K}]$ vs. Distance from laser spot centre $[\mu \mathrm{m}]$ plot at laser fluence (A) $2.4614 \mathrm{~J} / \mathrm{cm}^{2}$, and (B) $0.8208 \mathrm{~J} / \mathrm{cm}^{2}$. The possible (TiO. 716 ) 3.76 formation temperature range 
is marked between the dotted lines in plot (A) and (B). Temperature [K] vs. Depth $[\mu \mathrm{m}]$ plot at laser fluence (C) $2.4614 \mathrm{~J} / \mathrm{cm}^{2}$ and (D) $0.8208 \mathrm{~J} / \mathrm{cm}^{2}$. The possible (TiO. 716 ) 3.76 formation temperature range is indicated between the dotted lines in plot (C) and (D), with the extrapolated $\mathrm{x}$-intercept showing the maximum depth up to which it can form. .......................................... 129 


\section{Chapter 1}

\section{Introduction}

Absorption of photons is desirable in diverse applications. Generally, this involves the transfer of energy from photons to electrons of the absorbing material. The range of the solar spectrum, from which photons need to be harvested, is application specific. Selective absorption of photons from sections of the visible light range is adequate for substances like pigments, dyes and photonic crystals. Photo-electronic devices, like photo detectors and diodes, are often designed to be sensitive to narrow wavelength ranges, and can convert the energy of those photons to an electric current. Solar cells and various solar energy harvesting devices on the other hand, desire materials that have a steady photon absorption over a broader range of wavelengths, and can harvest as many photons as possible.

Numerous techniques have been applied to increase the absorption of materials. Surface texturing and destructive interference coatings have been utilised to reduce reflection and to trap more photons. They attempt to enhance absorption by coupling and trapping of light through total internal reflection [1]-[5]. To collect infrared radiation, the process of up-conversion was first implemented for infrared detectors, [6]. It was then explored for photovoltaic applications where typical up-convertors consisting of active ions set in a host material were integrated into solar devices [7]. With the advent of nanoscience and nanotechnology, attempting to harvest photons over a broader wavelength using nanomaterials has become promising. 


\section{$1.1 \mathrm{TiO}_{2}$ Nanomaterials}

Nanomaterials based on numerous metals, metal oxides and semi-conductors like Si (both in crystalline and amorphous form), $\mathrm{TiO}_{2}, \mathrm{CdS}, \mathrm{CdSe}$, CdTe, CIGS, $\mathrm{ZnO}$ and $\mathrm{CuO}$ have all been applied as photon absorber materials [8]-[10]. But Titanium Dioxide in particular, has gained much interest after the reporting of its excellent photo-catalytic behaviour [11], [12]. Titanium by itself, (Atomic Weight: 47.88), is found abundantly in nature and is known for its high strengthto weight ratio, low levels of toxicity and good corrosion resistance [12]. It has five naturally occurring isotopes and two allotropic forms. Over the years, its reduced manufacturing costs has propelled the usage of titanium compounds in numerous applications.

Titanium-dioxide, $\mathrm{TiO}_{2}$, the naturally occurring oxide of titanium occurs mainly in three different forms: rutile (tetragonal), anatase (tetragonal) and brookite (orthorhombic). It is an $n$-type semiconductor due to its oxygen deficiency [13]. It was and continues to be cheap, readily available with low levels of toxicity, and is resistant to corrosion [12]. As mentioned earlier, its been widely used as a pigment due to its high refractive index and brightness since decades. However, since the discovery of the ability of $\mathrm{TiO}_{2}$ to decompose water and produce hydrogen under ultraviolet (UV) irradiation in 1972, a wide interest was generated into the research of $\mathrm{TiO}_{2}$ nanomaterials [11]. $\mathrm{TiO}_{2}$ based nanomaterials are synthesised using a number of methods like Sol/Sol-Gel methods, physical and chemical deposition methods (PVD \& CVD), oxidation methods and hydro/solvo-thermal method [14], [15]. The method of manufacturing plays a significant role in determining its properties. 


\subsection{Applications of $\mathrm{TiO}_{2}$ Nanomaterials}

Due to emergence of new physical and chemical properties at the nanometric scale, $\mathrm{TiO}_{2}$ nanomaterials of various shapes and sizes have been applied in photovoltaics, photocatalysis, photonic crystals, sensors, UV blocks, pigment and paints [16].

With a large band gap value and a high absorption in the $\mathrm{UV}$ range, $\mathrm{TiO}_{2}$ nanomaterials have been used in UV protection applications [17], [18]. These include sunscreen creams, textiles and plastic films [18]. Its use in pigments and paints comes from the fact that it has a high refractive index value of 2.70, which makes it better equipped to produce greater opacity [19]. As such, its colour is controlled by its particle size [20].

The photocatalytic property of $\mathrm{TiO}_{2}$ arises due to the photocatalytic oxidation reactions that occur after the absorption of a photon, of energy equal to its band gap or higher. This causes an excitation of an electron from valence to conduction band, thereby leading to the generation of charge carriers in the form of electrons and holes [21]. Such charge carriers then help in the degradation of chemicals adsorbed on its surface [22]. The use of $\mathrm{TiO}_{2}$ in photocatalysis is again driven by its UV absorption capability and has been applied in the development of self-cleaning materials, degradation of pollutants and bacteria killing agents [23]-[26]. With the promise held by $\mathrm{TiO}_{2}$ nanomaterials by the virtue of their photocatalytic performance, many studies have been done to overcome the limitation set by its narrow UV absorption spectrum. To exploit the properties of $\mathrm{TiO}_{2}$ in numerous photon harvesting applications, broadening of the absorption capability of $\mathrm{TiO}_{2}$ to the visible spectrum is essential. This has led to many efforts being made to modify the optical activity and hence absorption of $\mathrm{TiO}_{2}$, which will be discussed in the next section. 


\subsection{Efforts to Enhance the Optical Absorption of $\mathrm{TiO}_{2}$}

\section{Nanomaterials}

Like any semiconductor, the optical absorption of $\mathrm{TiO}_{2}$ is determined by its electronic band structure, especially its lowest energy transition. When this transition is direct, which corresponds to electrical dipole allowed transitions, the absorption is characterised by sharp excitonic peaks. Indirect transitions on the other hand require phonon assistance and have weak absorption with no sharp peaks. Since $\mathrm{TiO}_{2}$ has a large bandgap value, the lowest energy transitions occur in the UVnear UV region.

Efforts made to enhance the optical activity of $\mathrm{TiO}_{2}$ nanomaterials by broadening its absorption capability to visible wavelengths can be broadly classified into three categories. This is illustrated in Figure 1.1: Doping, Sensitisation and coupling [22].

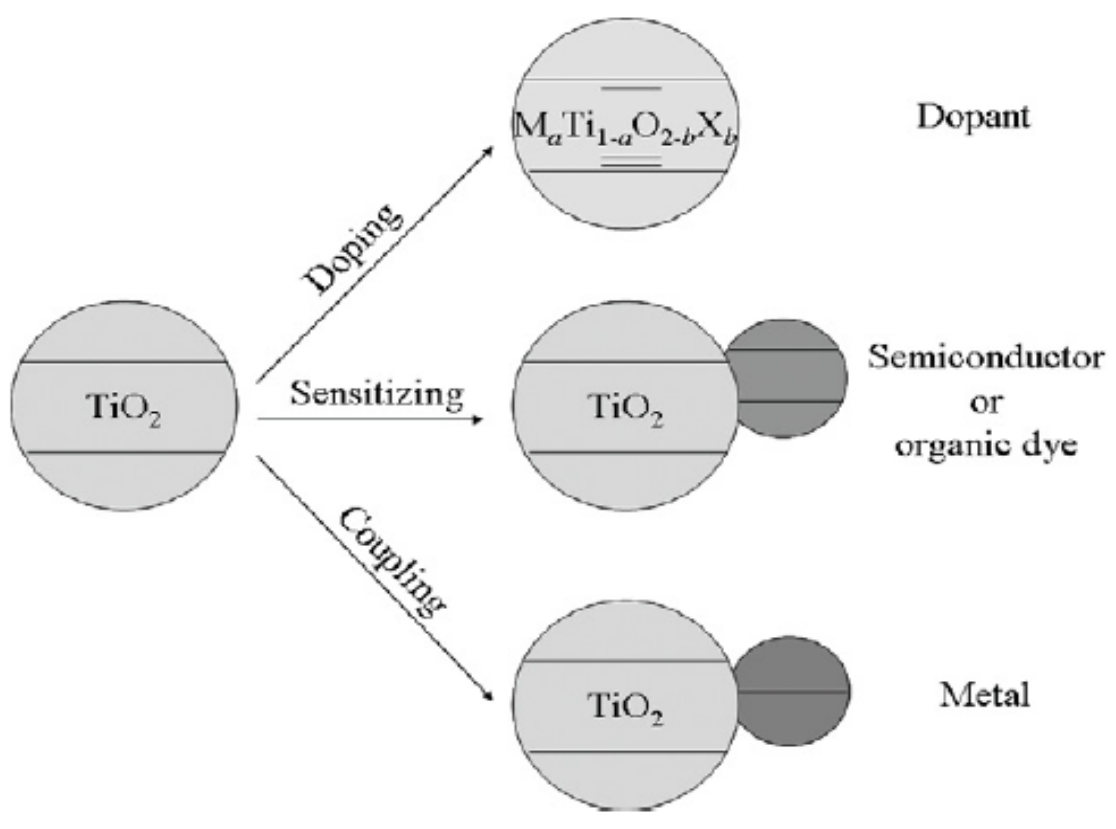

Figure 1.1: Illustration of the modification paths for $\mathrm{TiO}_{2}$ nanomaterials [22]. 
Doping of $\mathrm{TiO}_{2}$ has been done with both metal and non-metals to induce charge transfer between the d-electrons of the dopant to conduction or valence band of $\mathrm{TiO}_{2}$ and thereby cause a red shifted visible light absorption [22]. This can help generate more electronic states above the valence band of $\mathrm{TiO}_{2}$. Non-metals like Carbon, Nitrogen, Sulphur and transition metals like Vanadium, Chromium, Nickle and Iron have all been used as dopants, with an effort to increase the absorption in the visible region [27]-[32]. Figure 1.2 gives the red shifting of the absorption spectrum achieved with different kind of doping.
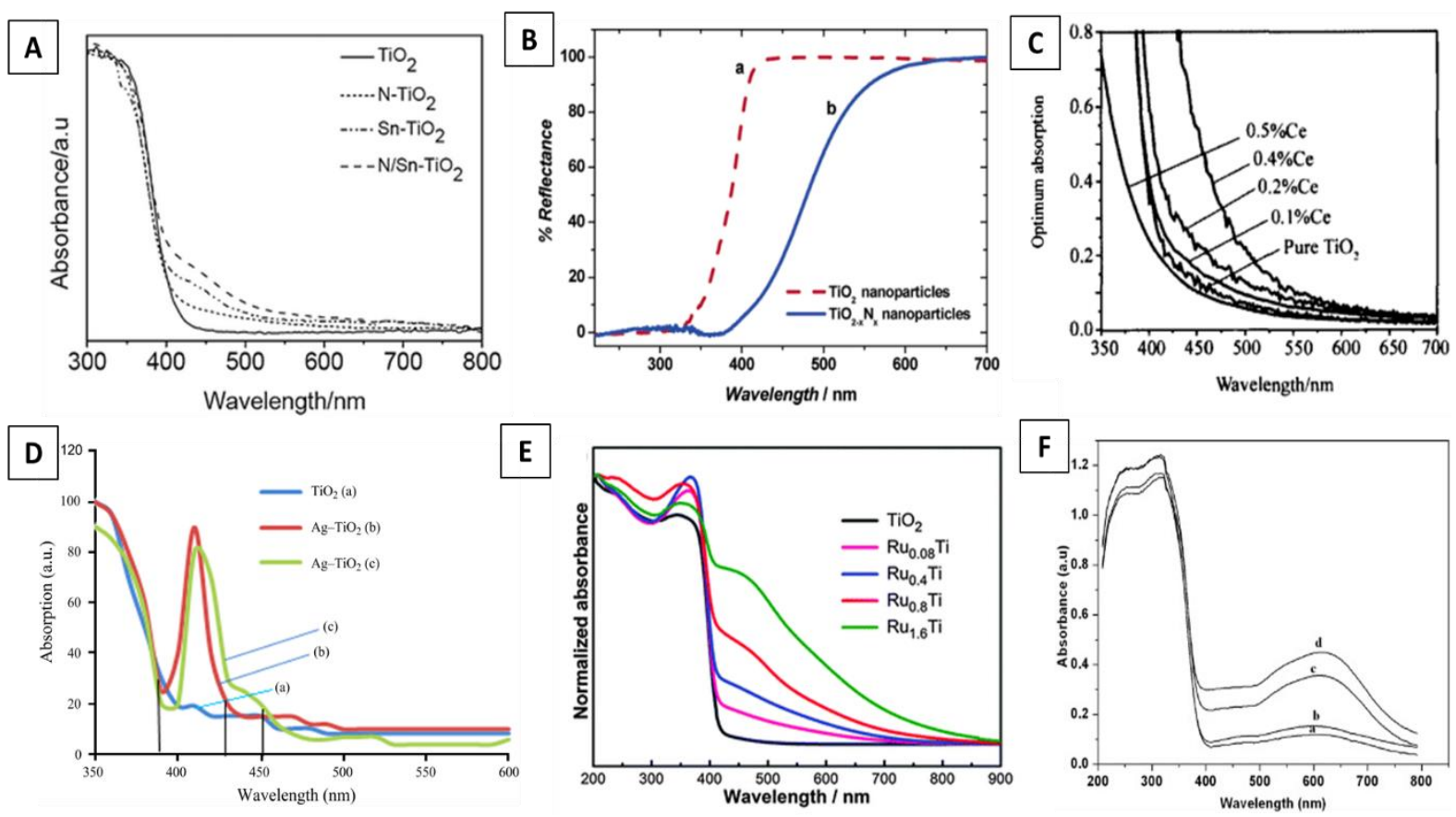

Figure 1.2: Absorption/Reflectance spectrum $\mathrm{TiO}_{2}$ nanomaterials doped with (A) Nitrogen (N), Tin(Sn) [33], (B)

Nitrogen [27], (C) Cerium (Ce) [34], (D) Silver (Ag) [35], (E) Ruthenium (Ru) [36], and (F) Gold (Au) [37].

Sensitizing of $\mathrm{TiO}_{2}$ with semiconductors like $\mathrm{PbS}$ nanoparticles, $\mathrm{Ag}$ nanoparticles and various organic dyes is another method employed to improve its optical absorption. This has led to improved photo conductance in visible regions, with dyes especially attracting more attention [38], [39]. Organic conjugated polymers are also being studied for $\mathrm{TiO}_{2}$ sensitisation [40]. These 
sensitizers when adsorbed onto the $\mathrm{TiO}_{2}$ surface, are excited upon irradiation and act as electron injectors into $\mathrm{TiO}_{2}$. The thermal and photochemical instability associated with such conjugated polymers however, has restricted their usage [41]. This propelled research of Quantum Dots (QDs) made of $\mathrm{PbSe}, \mathrm{CdTe}$,InAs and other inorganic materials to sensitise $\mathrm{TiO}_{2}$ [42]-[45].

The third method of $\mathrm{TiO}_{2}$ modification for improving its optical properties is the coupling of the collective oscillations of electrons in the conduction band of the surface of the metal nanoparticles, with those in the conduction band of $\mathrm{TiO}_{2}$ [22]. This phenomenon, known as the Surface Plasmon Resonance (SPR) has later been used to enhance the absorption of the nanomaterial developed in this dissertation. This study is described in Chapter 6. Like sensitizers, Surface Plasmons are photo excited in the visible region and act as electron sinks. Noble metals such as gold, silver and silver halides have been used to enhance the visible light irradiation of $\mathrm{TiO}_{2}$ [46]-[49]. Figure 1.3 given below shows the enhancement in absorption achieved by inducing the plasmon resonance phenomenon.
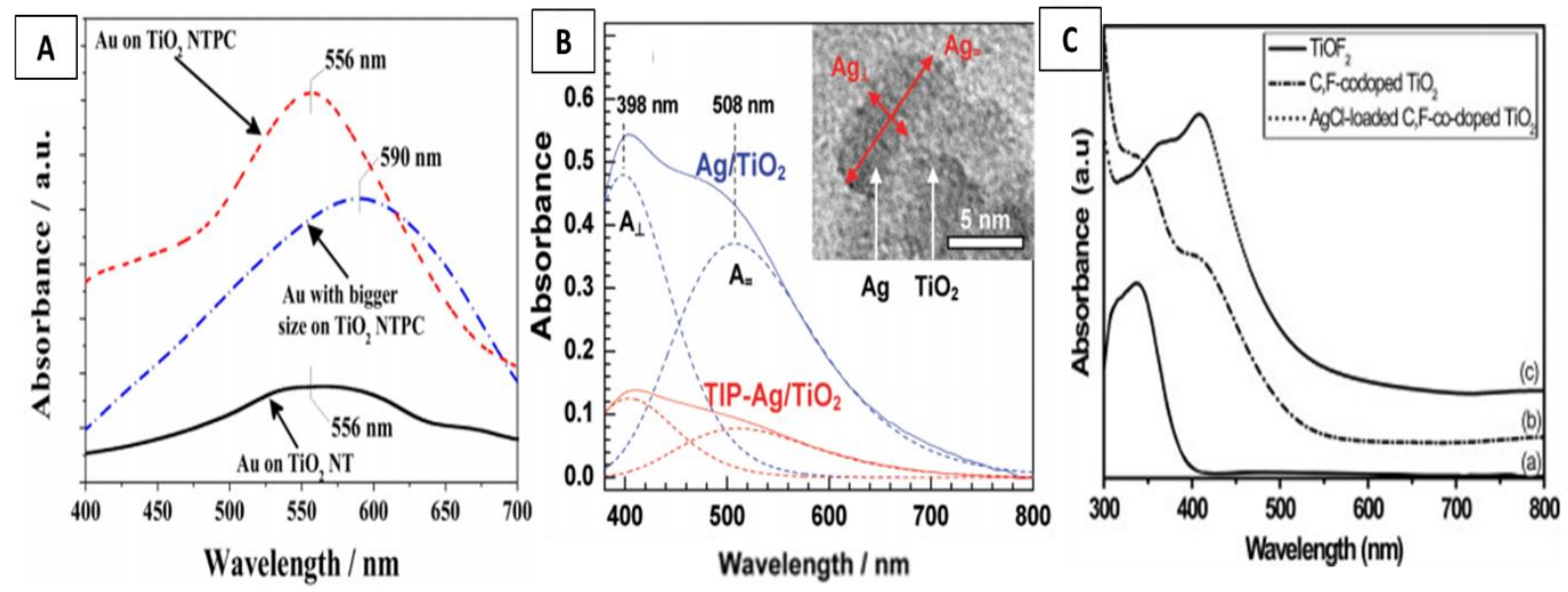

Figure 1.3: Plasmon absorption spectrum of $\mathrm{TiO}_{2}$ loaded with (A) Gold (Au) [50], (B) Silver (Ag) [51], and (C) Absorption spectrum of $\mathrm{TiO}_{2}$ doped with Carbon and Fluorine and loaded with a Silver halide $-\mathrm{AgCl}$ [52]. 
More recently, efforts have been made to enhance the optoelectronic properties of nanostructured $\mathrm{TiO}_{2}$ via 'self-doping' This is done by incorporating surface disorders and defects like titanium interstitials $\left(\mathrm{Ti}^{3+}\right)$ and oxygen vacancies in its structure [53]-[56]. Band gap reduction or induction of mid gap states takes place which improve optical absorption [57]. Such surface defects and oxygen vacancies also help in increasing the donor density by acting as electron donors and boost charge transportation [57]. Thereby functional properties like electronic structure and catalytic performance are much more effectively influenced and improved than by element doping [58]. This is because, conventional doping is afflicted by many issues. While metal doping causes recombination of charge carriers, heavy doping with non-metals is often challenging, leading to limited visible absorption [59], [60]. Furthermore, thermal instability and potential leakage of such dopants can be an environmental hazard [61]. Thus 'self doping' strategies in $\mathrm{TiO}_{2}$ nanomaterials are more advantageous and can help overcome such problems. Self doping is also favoured as it helps retain the intrinsic crystal structure of $\mathrm{TiO}_{2}[62]$.
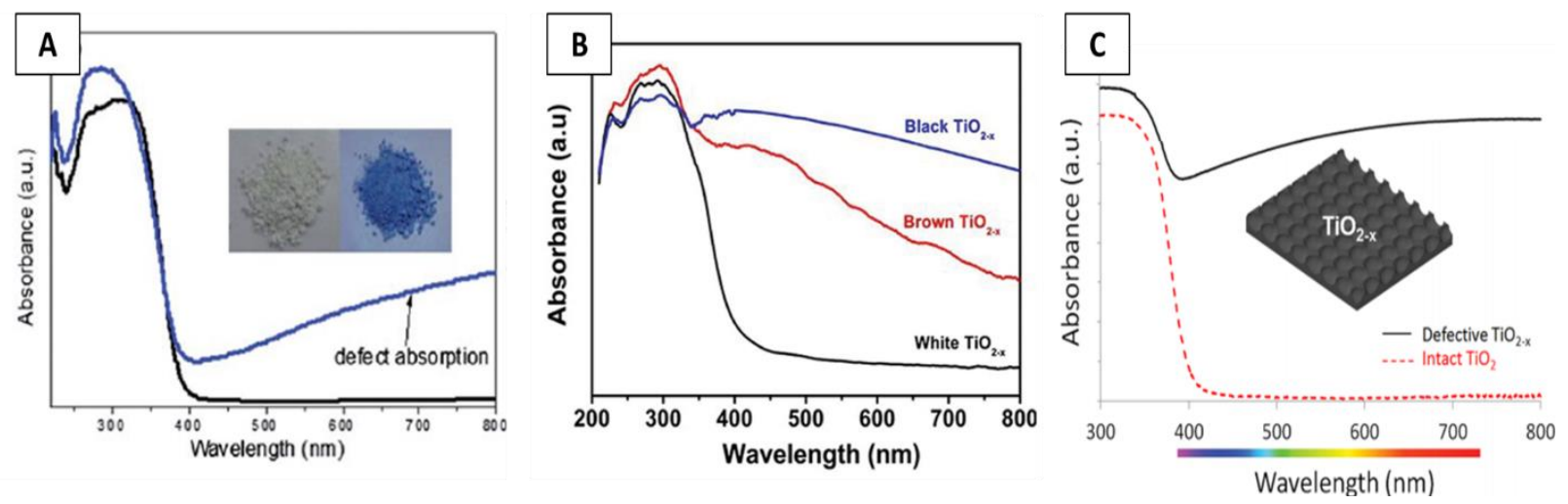

Figure 1.4 Absorption spectrum of various 'self doped' $\mathrm{TiO}_{2}$ nanomaterials (A) stable blue $\mathrm{TiO}_{2-\mathrm{x}}$ [63], (B) black/brown/white defective $\mathrm{TiO}_{2-\mathrm{x}}[64]$, (C) black defective $\mathrm{TiO}_{2-\mathrm{x}}[65]$.

Latest works on 'self doped' $\mathrm{TiO}_{2}$ have resulted in the formation of $\mathrm{TiO}_{2}$ nanomaterials which can absorb the entire or part of visible light causing them to appear either black or of different colours 
like yellow, blue, green or brown [54], [66]-[68]. This is due to the additional absorption bands created due to defects and vacancies. Various reduction (from $\mathrm{TiO}_{2}$ ) and oxidation methods (from low valence state $\mathrm{Ti}$ species) have been employed to form self-doped $\mathrm{TiO}_{2}$, which are often complicated and involve high costs [61]. Also, most of these methods result in the formation of low concentration of $\mathrm{Ti}^{3+}$ doping, often limited to surface, which either easily oxidise or show less visible absorption due to inhibition of electron mobility [62]. The absorption spectrum of few such 'self doped' $\mathrm{TiO}_{2}$ nanomaterials have been shown in Figure 1.4. Chapter 7 shows the work done in promoting the formation of high degree of oxygen vacancies and defects in the nanomaterial developed in this dissertation. The simple one step laser synthesis method is utilised to 'self-dope' and thereby disorder engineer the unique nanomaterial.

\subsection{Research Motivation}

As discussed in the previous section, many endeavors have been made by the scientific community to broaden the absorption spectrum of $\mathrm{TiO}_{2}$ nanomaterials into the visible region. However, it has been a challenge to extend the absorption capability of $\mathrm{TiO}_{2}$ throughout the visible range and beyond into the near infrared (NIR) spectrum. The entire visible and NIR regions constitute about $44 \%$ and $40 \%$ respectively of the solar irradiation reaching the ground level when the sun is at its zenith [69]. It thus becomes imperative for any photon absorbing material to be able to maximise the harvesting of this large pool of photons. $\mathrm{TiO}_{2}$-based nanomaterials have shown preeminence as photon absorbers that can be applied in numerous disciplines, especially in photovoltaics. This is due to their abilities in effectively generating charge carriers through UV photon excitation, by the virtue of their superior photocatalytic properties. As such, there is 
considerable incentive in extending the absorption spectrum of similar types of nanomaterials into the vis-NIR region.

A simple one step method of fabricating three dimensional (3D) nanostructures via ultrashort laser synthesis has been previously demonstrated in the Laser Micro Nano Fabrication Lab at Ryerson University. Earlier studies conducted in the lab on nano Si showed improved absorption in the visible region [70]. Oxides of Titanium, exhibiting semi-conductor characteristics themselves, like Silicon were hoped to show an improved absorption enhancement via such a method. The

equipment and expertise to generate a Titanium Oxide based nanomaterial was thus already available. Using the basics from those methods and by varying the laser material interactions, nanomaterials can be developed whose physical and chemical properties can be manipulated on a nano level. Leveraging such methods, the aim of this research has been to generate a nanomaterial based on Titanium Oxides, and to engineer its properties for achieving enhanced and broadened photon absorption.

\subsection{Research Objectives}

The main objectives of this research are given below:

- To develop a nanomaterial composed of Titanium Oxides that is capable of enhanced photon absorption over a broader range of solar spectrum.

- To improve photo absorptive capabilities of the nanomaterial developed, by tuning its physiochemical properties through the utilisation of methods like plasmon resonance and disorder engineering. 


\subsection{Dissertation Organisation}

So far, Chapter 1 has introduced the diversity in applications of photon absorption materials. A literature review of applications and limitations of the photo absorptive capability of $\mathrm{TiO}_{2}$ has been presented. This has been followed by a summary of efforts made by other researchers to overcome such limitations, and a discussion of research motivations. This dissertation's objective, as stated in section 1.5 , is to potentially attain 'full spectrum' photon absorption.

Prior to discussing the contributions of this dissertation, Chapter 2 and Chapter 3 lay out several preliminary materials. The method used to synthesize nanomaterials based on the Oxides of Titanium is laid out in Chapter 2. Following this is an explanation of the physics behind the interaction of ultrafast laser pulses with the target material, along with the various mechanisms leading to its formation. A mathematical analysis of surface-temperature calculation of the target material is also presented, and a discussion of the mechanism behind the nanostructure's formation and growth is given. Chapter 3 lays out the experimental setup used for the material synthesis, i.e., the femtosecond laser fabrication system, and the material used for research studies. Next, characterisation methods for the analysis of morphological, chemical and optical properties are discussed, which have later been used on the nanomaterials developed in this dissertation.

Chapter 4 shows the phase functionalization of Titanium for the formation of a novel multiphase Oxide of Titanium, which has an enhanced and broadened photon absorption capability. To better understand the composition of this novel Oxide and the conditions leading to its formation, material and phase characterisation studies have been carried out. This chapter concludes with measurements of absorption enhancement achieved by the phase functionalization of Titanium, and shows a calculation its band gap. 
The incorporation of the above mentioned multiphase Oxide of Titanium in the form of a 3D selfassembled nano network is presented in Chapter 5. The formation mechanism of this nano $\mathrm{TiO}_{\mathrm{x}}$ network is elucidated, along with surface and material characterisations. A study has also been performed by varying the laser synthesis conditions, and observing the changes in size distribution of the nano networks and their absorption spectrum.

Building on this study, Chapter 6 delves into the modification of the nano $\mathrm{TiO}_{\mathrm{x}}$ network to improve its ability to absorb photons. The induction of the surface plasmonic effect on the self-assembled nano $\mathrm{TiO}_{\mathrm{x}}$ network is demonstrated through its hybridisation with gold and a gold/palladium alloy. Illustrated in this chapter, are the studies that were performed to examine the impact of several factors (like size, composition and degree of hybridisation) on the photon absorption capability of this self-assembled 3D multiphase nano $\mathrm{TiO}_{\mathrm{x}}$ network.

Chapter 7 takes this a step further with the synthesis of a disordered multiphase nano $\mathrm{TiO}_{\mathrm{x}}$ network, to promote the formation of defects and vacancies, achieved through alterations made in the plume kinetics during the laser synthesis process. Characterisation of its surface and material properties is presented, followed by an analysis of defects. Spectroscopy studies of this disordered material are also offered, which include the absorption, emission and Raman spectrum results.

Chapter 8 then gives a brief discussion on the extended potential applications of the multiphase nano $\mathrm{TiO}_{\mathrm{x}}$ network. Finally, Chapter 9 gives a summary of the entire dissertation, along with concluding remarks on future research. Throughout the thesis, suitable references that touch upon the theoretical aspects have been provided from literature, to cross reference and/or support the research study results. 


\section{Chapter 2}

\section{Laser Synthesis Mechanism}

\subsection{Physics of Ultrafast Laser-Material Interaction}

Femtosecond (fs) lasers (also known as ultrashort pulse (USP) lasers) emit laser pulses with duration of few femtoseconds to a couple of hundred femtoseconds. They are usually generated using the passive mode locking technique. The femtosecond laser deposition timescale is less than the time required for the atomic relaxation (thermalization) of the specimen. As such, the pulse duration is much shorter than the time of transfer of energy between the free electrons and material lattice. This results in the electrons absorbing the energy and the lattice being left cold. Threfore, high temperatures and pressures are seen within a very short depth.

Femtosecond laser pulses can thus cause optical breakdown of the material. High laser intensities result in a non-linear absorption of energy, leading to processes such as multi-photon ionisation. Thereby, upon laser irradiation, the target material undergoes significant changes. Its response is a function of local heating and cooling rates and the maximum temperature reached.

The 'ablation threshold' refers to the minimum energy of the laser required for starting the ablation (atomic and molecular separation or displacement) of the material. When the incident laser beam energy is smaller than the melting and ablation threshold of the target material, rapid phase transformation and restructuring of the crystal lattice can occur at such high temperatures [71]. 
The material used in this study, Titanium, was subjected to this kind of thermal activation process to develop a novel multiphase Titanium Oxide. This study will be discussed in Chapter 4.

When irradiated with a laser energy greater than its ablation threshold, the target material undergoes a material removal process. The energy dissipation and the corresponding material removal after laser interaction can occur by two different mechanisms: (a) Thermal Vaporization, (b) Coulomb Explosion. In thermal vaporization, the collisions between the photons and electrons cause a significant increase in local temperature leading to vaporization of the material. This usually occurs way above the ablation threshold leading to strong ablation. In Coulomb Explosion, excited electrons escape bulk material and form a strong electric field, which causes the ions to be pulled out in the impact area. This occurs near the ablation threshold leading to a gentle ablation. The ionization generated by the above mechanisms continues until critical dense "plasma" is created, above the sample surface. This "cloud" of plasma thus generated, called as the plasma plume, consists of clusters of electrons, ions and target material particles [72]. In metals, the plasma plume tends to form at lower laser energies than in dielectrics due to the presence of a large number of surface electrons. Sometimes the "incubation effect" caused by the thermal influence of previous laser pulses on the ablation performed by the ensuing laser pulses, affects the ablation rate. The interaction of the species ejected from the target with that of the ambient gas influences the formation and growth of nanoparticles. In laser pulses of femtosecond range, secondary interactions between the ablated species and the laser beam do not take place, and the kinetic energy of the expanding plume is large [73]. The angular distribution of the ablated species and the kinetic energy of laser plasma plume influence the nanostructure formation and growth [73]. The use of femtosecond lasers for the synthesis of nanostructures is advantageous for several reasons. Firstly, the pulse ends before the expansion and the laser does not interact with the ejected 
material [72]. This makes the study of hydrodynamics and thermodynamics possible without the consideration of any external disturbances [72]. Secondly, the density of the material remains unchanged, so the initial thermodynamic properties are easily known [72]. And finally, due to its greater fluence, the femtosecond laser has the ability to heat any material to a solid density plasma state with temperatures and pressure above the critical point [72].

\subsection{Surface Temperature of Target Material}

The surface temperature of the target material at the cessation of the laser material interaction is a function of its thermal diffusivity and light absorption. The following method proposed in [74] for the femtosecond laser system used in this dissertation, estimates the maximum temperature a target material attains when heated by a train of femtosecond laser pulses. The heat loss due to radiation and plume expansion is not considered for simplicity. It is assumed that the energy of the laser is absorbed much before the complete penetration of the heat wave happens. The surface temperature obtained at the end of a laser pulse, $T_{\mathrm{m}}$, at the center of the laser spot area is given by the equation [74], [75],

$$
T_{\mathrm{m}}=\frac{\left(\frac{2}{\pi}\right)^{\frac{1}{2}} I\left(a t_{\mathrm{p}}\right)^{\frac{1}{2}}}{\kappa}
$$

where $t_{\mathrm{p}}$ is pulse duration (pulse width), $a$ is the thermal diffusion coefficient, $\kappa$ is the heat conduction coefficient and $I$ is the absorbed laser intensity, which for a wavelength of $1040 \mathrm{~nm}$ (wavelength of the laser beam generated by the fabrication system outlaid in section 3.1 and used for the generation of the nanomaterial in this thesis) was estimated to be [76]: 


$$
I=A \frac{4 P}{\pi d^{2} t_{\mathrm{p}} f}
$$

where $A$ is the laser absorption coefficient, $P$ is the laser power, $f$ is the pulse frequency or repetition rate and $d$ is the laser spot diameter.

As described in [74] and [75], Equation 1, applicable for a single laser pulse, is then used to arrive at a second equation to calculate the average surface temperature $\bar{T}_{\mathrm{n}}$ at the center of the laser spot after ' $n$ ' number of pulses [74], [75],

$$
\bar{T}_{\mathrm{n}}=2 \alpha \frac{\left(1-\frac{2}{3} \alpha\right)}{\left(1+\alpha^{2}\right)} \cdot \frac{T_{\mathrm{m}}}{(1-\alpha)}\left(1+\frac{\alpha^{n}-\alpha}{n(1-\alpha)}\right)
$$

where $T_{\mathrm{m}}$ calculated in equation 1 , is the temperature obtained at the end of the laser pulse at the laser spot center, and $\alpha$ is a constant ratio given by $\left[t_{\mathrm{p}} \cdot f\right]^{1 / 2}$. Figure 2.1 shows this variation in temperature of the target surface at a single laser spot centre, when heated by a series of laser pulses [75].

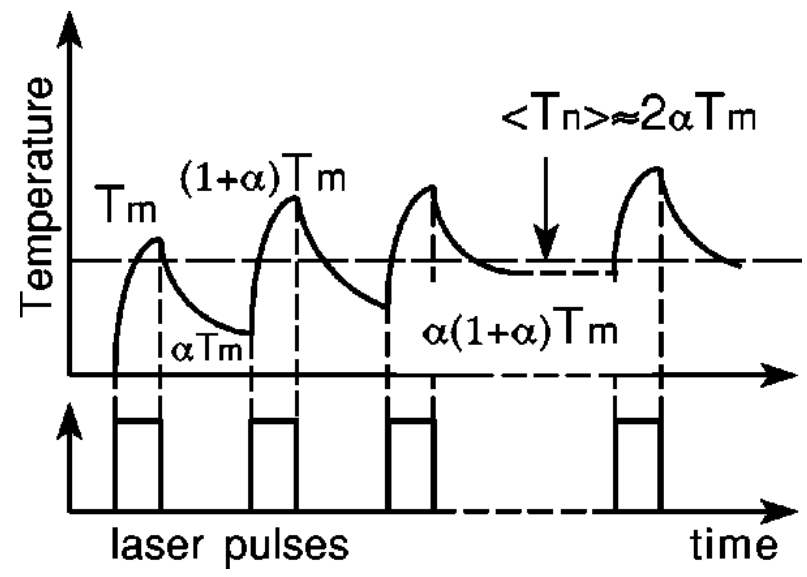

Figure 2.1Temperature of the laser spot area on the target surface upon irradiation with a series of laser pulses [75]. 
This equation 3 is eventually used to calculate the theoretical average surface temperature at the laser spot center obtained for different laser parameters. The value of $n$ in equation 3 was substituted to be equal to the value of $N_{\text {eff }}$ calculated for each specific laser parameters used. $N_{\text {eff }}$ is the effective number of laser pulses which interact with a surface area equivalent to that of the laser spot area, at a given pulse repetition rate and scanning speed. It can be calculated by equation 4 given below [77].

$$
N_{\text {eff }}=\frac{\left(\sqrt{\frac{\pi}{2}}\right)(\mathrm{d} / 2) f}{V}
$$

where $d$ is the laser spot radius, $f$ is the repetition rate and $V$ is the scanning speed of the laser beam on the surface of the target material. Furthermore, the laser fluence, $L_{f}$, defined as the as the optical energy delivered per unit area $\left(\mathrm{J} / \mathrm{cm}^{2}\right)$ was calculated through out the thesis using the equation [70]:

$$
L_{f}=\frac{P}{f \pi(\mathrm{d} / 2)^{2}}
$$

\subsection{Nanostructure Formation and Growth}

As the plume expands and diffuses into the surrounding medium, adiabatic cooling takes place leading to vapour condensation and finally nanostructure formation. The time required for their formation is much shorter than the time for which the plume exists [78]. And this difference in time determines the growth of the nanostructures [78]. The laser plasma plume temperature and the density of the ionic species inside the plume both increase with laser intensity until saturation [72], [79]. The plasma species collide with molecules of the surrounding medium, causing 
aggregation of atoms and molecules which eventually form nuclei. The expansion of the plume can be decelerated by introducing different background gases at various pressures. Apart from compressing and restricting the plasma plume, it can lead to increased chemical reactivity between the plume species and the gas molecules, forming various atomic and diatomic species [73].

The different stages of the vapour condensation mechanism involve the nucleation, growth and finally cooling. Nucleation occurs when the barrier energy due to surface tension is overcome by the surface energy. This surface energy is related to temperature and size. The particle formation energy as a function of radius $\Delta G_{\text {tot }}(r)$ is given by the equation [80]:

$$
\Delta G_{\mathrm{tot}}(r)=4 \pi r^{2} \sigma-\frac{4}{3} \pi r^{3}\left(n_{a} \Delta \mu\right)
$$

where $r$ is the nuclei cluster radius (assumed to be spherical), $\Delta \mu=k T \ln (S)$ is difference in chemical potential between condensed and uncondensed phases, $\sigma$ is the surface tension, $n_{a}$ is the atom number density, $k$ is the Boltzmann constant, $T$ is absolute temperature and $S$ is entropy. Rate of nucleation is a function of super cooling and the rate of volume growth once the nuclei is formed, is proportional to its area cross section [81], [82]. Both these factors determine the size of the nanostructures formed which are usually below $100 \mathrm{~nm}$. These newly formed nanostructures continue to grow and agglomerate until they cool down, which is governed by the surrounding gas medium. Furthermore, the rate of cooling influences the crystal behaviour of the nanostructure formed. At higher cooling rates, metastable amorphous can nucleate but at lower cooling rates, more stable crystalline phase can form [83]. Also, such crystalline nanostructures formed would tend to be larger, as they had longer time to form (due to lower cooling rates) [83]. This influence of cooling rate on the crystallinity of the nanostructure formed is observed and discussed further in Chapter 7. 


\subsection{Summary}

This chapter provided the basics physics behind the interaction of a femtosecond laser pulse with a target material. The formation of the laser plasma plume and its influence on the formation of nanostructures is elucidated. The equation to calculate the surface temperature of the target material, once the laser irradiation stops, at the laser interaction spot has been given. The chapter closes with an explanation of how the vapour condensation mechanism leads to nanostructure formation and the factors which influence its characteristics. 


\section{Chapter 3}

\section{Experimental Setup and Procedure}

This chapter deals with the experimental setup of the laser fabrication system, materials and methodology employed for the experiments and the various characterisation methods used for the studies in this dissertation. All experiments utilised the Megahertz femtosecond laser system located at the Micro and Nano Fabrication Research Laboratory at Ryerson University, Toronto, Canada.

\subsection{Laser Fabrication System}

All the experiments were carried out using a direct diode pumped, Yb-doped fiber amplified laser system (Clark-MXR Inc. IM-PULSE series ultrashort pulse laser), to generate a femtosecond pulse laser beam of pulse repetition rate (frequency rate) ranging from $200 \mathrm{kHz}$ to $26 \mathrm{MHz}$. The laser beam with a diameter of approximately $4.5 \mathrm{~mm}$ was expanded by using a combination of planoconvex $(f=-100)$ and plano-concave $(f=200)$ lenses. To circular polarize the beam, a quarter wave plate was placed in its path and the beam was reduced to $8 \mathrm{~mm}$. A two-axis computer controlled Galvano scanner then scanned the laser beam normally on the sample surface. The beam was then focussed normally on to the surface of the target material by using a telecentric lens of focal length $63.5 \mathrm{~mm}$. The laser beam has a Gaussian shaped beam profile and a central wavelength of $1040 \mathrm{~nm}$ with a calculated highly focused spot diameter of about $10 \mu \mathrm{m}$. Different scanning speeds were used to scan the surface of the target material, at a constant average power 
of $16 \mathrm{~W}$. The range of pulse separation varied from 250 fs to $10 \mathrm{ps}$. Repetition rate values were chosen to be $4 \mathrm{MHz}, 8.36 \mathrm{MHz}, 12.54 \mathrm{Mhz}$ and 26.07 MHz. The laser fluence values calculated using equation 5 at these repetition rates was $5.14 \mathrm{~J} / \mathrm{cm}^{2}, 2.46 \mathrm{~J} / \mathrm{cm}^{2}, 1.64 \mathrm{~J} / \mathrm{cm}^{2}$ and $.82 \mathrm{~J} / \mathrm{cm}^{2}$ respectively.

The laser spot overlap (LSO) percentage was determined to get a better understanding of laser material interaction. The LSO formula is given by [84]:

$$
\operatorname{LSO}(\%)=\left(1-\frac{v}{f d}\right) \times 100 \%
$$

where $v$ is the scanning speed $\mathrm{mm} / \mathrm{s}, f$ is the rep. rate in $\mathrm{Hz}$, and $d$ is the laser beam size in $\mathrm{mm}$. In this thesis, the following conditions were used, as shown in Table 3.1. For all laser conditions, $d=0.01 \mathrm{~mm}, v$ and $f$ values were varied.

\begin{tabular}{|c|c|c|c|c|c|}
\hline & & \multicolumn{4}{|c|}{ Rep. Rate (Hz) } \\
\hline & & $4 \times 10^{6}$ & $8.36 \times 10^{6}$ & $12.54 \times 10^{6}$ & $25.07 \times 10^{6}$ \\
\hline \multirow{3}{*}{$v(\mathrm{~mm} / \mathrm{s})$} & 1 & $99.9975 \%$ & $99.9988 \%$ & $99.9992 \%$ & $99.9996 \%$ \\
\hline & 5 & $99.9875 \%$ & $99.9940 \%$ & $99.9960 \%$ & $99.9980 \%$ \\
\hline & 50 & $99.8750 \%$ & $99.9402 \%$ & $99.9601 \%$ & $99.9801 \%$ \\
\hline
\end{tabular}

Table 3.1: Calculation of LSO (\%) values 
The experimental set up has been shown in Figure 3.1 using a schematic drawing.
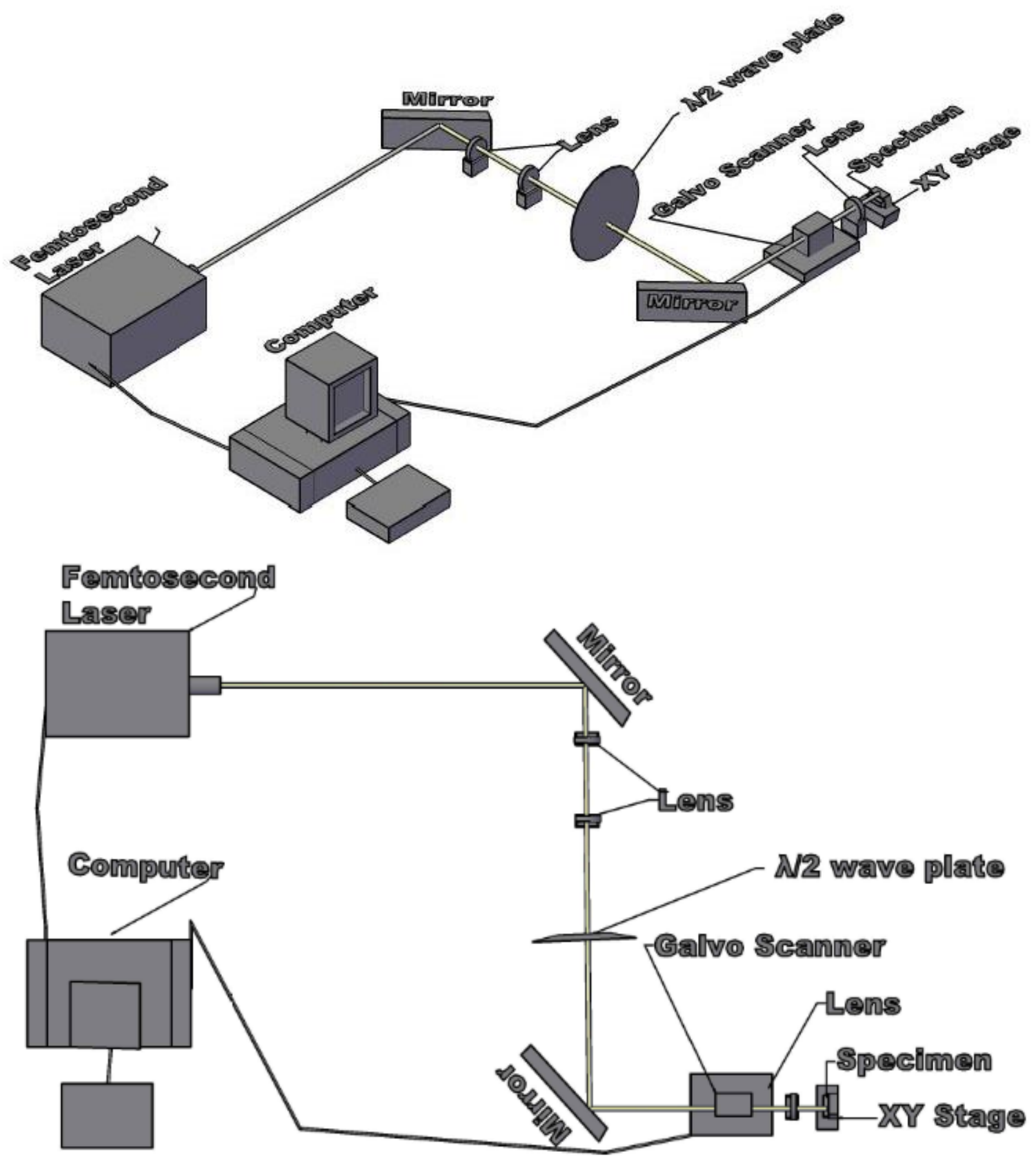

Figure 3.1: Isometric view (top) \& top view (bottom) of the experiment setup of the laser. 


\subsection{Material}

Titanium was used as the target material for all the experiments. It is available abundantly in nature and is known for its high strength-to weight ratio and corrosion resistance. It's a lustrous white metal with low levels of toxicity. It has five naturally occurring isotopes and two allotropic forms. The Titanium samples used for all the experiments were cut using a diamond saw and a coolant, from grade 2 (ASTM B265) pure Titanium bars of $2 \mathrm{~mm}$ thickness. Any possibility of the presence of minor surface defects and contaminants was removed by ground finishing and polishing, followed by ultrasonic cleaning in distilled water. Thereafter, the laser beam was scanned on the sample surface, under conditions of standard ambient temperature and pressure (SATP), at a temperature of $298.15 \mathrm{~K}$ and an absolute pressure of $0.98 \mathrm{~atm}$. The optical penetration depth of the laser beam (central wavelength of $1040 \mathrm{~nm}$ ) on the titanium substrate (absorption coefficient of titanium at this wavelength is $4.8295 e+5 \mathrm{~cm}^{-1}$ ) is $20.7 \mathrm{~nm}$. The thermal penetration depth of titanium for different laser pulse durations is approximately given as $\sim \sqrt{D} t_{\mathrm{p}}$ [85], where $t_{\mathrm{p}}$ is the laser pulse duration and $D$ is thermal diffusivity of titanium $\left(0.090123 \mathrm{~cm}^{2} / \mathrm{s}\right)$. The thermal penetration depth for laser pulse durations of $214 \mathrm{fs}, 714 \mathrm{fs}$ and $1428 \mathrm{fs}$ was calculated to be 1.39 $\mathrm{nm}, 2.54 \mathrm{~nm}$ and $3.59 \mathrm{~nm}$ respectively. Similarly, thermal penetration depth for a surface distance equal to the laser spot diameter, at different laser scanning speeds of $50 \mathrm{~mm} / \mathrm{s}, 5 \mathrm{~mm} / \mathrm{s}$ and $1 \mathrm{~mm} / \mathrm{s}$ was calculated to be $42.45 \mu \mathrm{m}, 134.25 \mu \mathrm{m}$ and $300.2 \mu \mathrm{m}$ respectively. 


\subsection{Characterisation Studies}

\subsubsection{Morphology Characteristics}

\subsubsection{Scanning Electron Microscope (SEM)}

The morphology of the functionalised Titanium and the nanomaterials was studied using an SEM (Hitachi SU1510). This type of analysis is a non-destructive way to test the material. The interaction between the high-energy electrons and the target surface is used to generate signals which provide information about the external morphology of the sample surface. Two dimensional (2D) images were acquired at numerous areas of the sample to provide special variation data.

\subsubsection{Field Emission Scanning ElectronMicroscope (FE-SEM)}

An FE-SEM (Hitachi SU8230) was used to perform ultra-high resolution imaging of the nanomaterials. The cold field emission source of electrons in an FE-SEM leads to minimal heating of the filament. This allows in obtaining better resolution images of the specimens. Through this a better understanding of the 3D structure of the nano networks was achieved. Furthermore, highly contrasting images of the plasmon hybridised nanomaterial were acquired, to better observe the extent of coverage due to hybridisation.

\subsubsection{Transmission Electron Microscope (TEM)}

The nanospheres constituting the nanomaterials were studied by doing the TEM analyses (Hitachi H 700 CTEM). The network was first detached from the substrate and immersed in isopropanol solution. For homogenous dispersion of the nanospheres in the solution, the samples were ultra- 
sonicated. This solution of dispersed nanospheres was placed on copper grids and allowed to dry. The TEM images were used to calculate the size of the nanospheres and a nanosphere size distribution plots were constructed from the data. The size of the nanospheres were calculated by using the public domain NIH Image program (developed at the U.S. National Institutes of Health and available on the Internet at http://rsb.info.nih.gov/nih-image/).

\subsubsection{High Resolution Transmission Electron Microscope (HR-TEM)}

A HR-TEM can generate an interference image by using both transmitted and scattered beam of electrons. This enables phase contract images to be created which can project $2 \mathrm{D}$ images of a crystal. The HR-TEM (JOEL 2010) was used to study the crystal orientations of the nanospheres in the nanomaterial and measure the d-spacing of the crystal lattice. The HR-TEM is also an ideal tool to image the defects in a crystal. The analysis was used to image the defects in the disordered nanomaterials and gain information about the type of defects in the crystal. Like the TEM analysis, the nanomaterials were detached and dispersed in solution. This was then placed on copper grids for analysis.

\subsubsection{Chemical and phase characterisations}

\subsubsection{Energy Dispersive X-Ray Spectroscopy (EDX)}

The elemental analysis of the functionalised Titanium and nanomaterials was done using the EDX (Hitachi SU1510). Elemental mapping using line and area scans was done to know the chemical composition of the nanomaterials. The EDX data gives peaks which corresponds to the elements present in the sample. This technique has a benefit of being non-destructive. 


\subsubsection{X-Ray Diffraction Analysis (XRD)}

The phase composition of the functionalised Titanium and nanomaterials was determined through XRD analysis (Bruker AXS D8 Discovery GADDS and X'pert PRO) using a CuKa radiation source, conventional theta/2theta diffractometer. XRD works on the principle of constructive interference between the sample and the incident X-ray beam. The subsequent diffraction of the X-rays by the sample is then detected and processed to obtain the XRD pattern. The average wavelength of the X-rays was $1.54184 \AA$ and the profiles were obtained with a $2 \theta$ range of 30-78 ${ }^{\circ}$ Compositional analysis was done using the Search-Match ${ }^{\mathrm{TM}}$ procedure to compare the generated pattern with a standard reference pattern. This was followed by the Rietveld fitting to know the approximate phase constituents of the samples.

\subsubsection{X-Ray Photoelectron Spectroscopy (XPS)}

XPS is a surface sensitive technique to determine the chemical state of the material. The characterisation of the functionalised Titanium and nanomaterials was done using an XPS (Themo Scientific K-Alpha). Aluminum k-Alpha X rays are used as beam source to excite the samples and cause emission of photoelectrons. An analyser then measures the energy of the photoelectrons. From this value of binding energy, information about the elemental identity and chemical state can be acquired. Average depth of penetration of source beams is $5 \mathrm{~nm}$. Both the Ti $2 \mathrm{p}$ and $\mathrm{O}$ s1 spectra of nanomaterial samples were obtained. 


\subsubsection{Absorption, Emission and Raman Spectroscopic}

\section{Characterisation}

\subsubsection{Vis-NIR spectrometer}

Optical absorption studies of all the material samples was performed using a reflection based method. A spectrometer with a deuterium-halogen source in the range of 200-1100 nm (AvaSpec2048 Fibre Optic Spectrometer). The spectrometer has a fibre optic connector, collimating and focussing mirror, along with a diffraction grating. The spectrometer was equipped with a 2048 pixel CCD detector array and calibrated to collect primarily diffused scattered light, as well as some specular scattered light. The background ambient light is subtracted as 'stray light' by taking an appropriate dark reference measurement. A single probe was used for both light emission and detection, giving a high level of accuracy to the analysis. The set of the Vis-NIR spectrometer has been given in Figure 3.2 [70]. In Chapter 7, the reflectance spectra of the disordered nanomaterials were obtained using a UV-vis-NIR spectrometer (Lambda 1050) with a $150 \mathrm{~mm}$ integrating sphere, for the calculation of band gap.

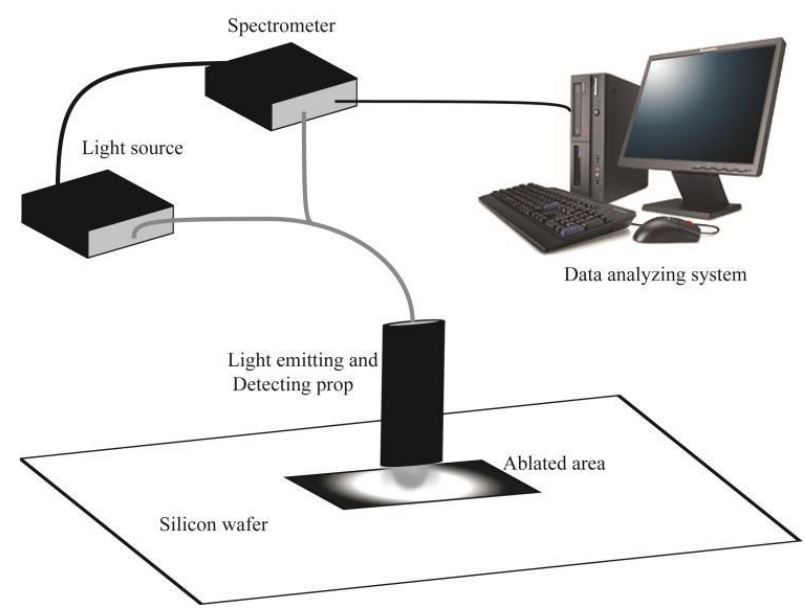

Figure 3.2 Set of the Vis-NIR spectrometer [70]. 


\subsubsection{Raman Spectroscopy}

The Raman spectra of the nanomaterials was measured using a Raman Spectrometer (BWTEK NanoRam) with laser beam wavelength of $785 \mathrm{~nm}$. The Raman spectra was used to characterize the material phase composition of the nanomaterials. To compare the Raman enhancement at multiple wavelengths of incident laser beam, a dual wavelength Raman microscope (BRUKER Optics SENTERRA) was used. Raman spectroscopy is a form of vibrational spectroscopy and is based on the principle of inelastic scattering of the laser incident on the sample. The interaction of the laser light with the molecules and phonons in the system cause the energy of the scattered laser photons to be shifted up or down. This shift is measured to give information of the vibration modes and thus characterise the phase composition of the samples.

\subsubsection{Spectrofluroscopy}

The Excitation and Emission spectra of nanospheres constituting the nanomaterials was measured at multiple excitation wavelengths varying in the range of $200-900 \mathrm{~nm}$, by using a spectroflurophotometer (Shimadzu RF-5301PC). The nanospheres were dispersed in aqueous solution for stability in measurements. The Emission spectra was acquired to indicate the photon excitation capability of the nanomaterials. Upon absorption of a photon of a certain wavelength by the sample molecule, its electronic state changes from ground to one of the excited states (often first excited state). The relaxation back to one of the lower vibration states then occurs, leading to emission indicating fluorescence (at longer wavelength emission) or up-conversion (shorter wavelength) process. 


\subsubsection{Summary}

This chapter describes the experimental set up used for synthesis of the nanomaterials for this study, followed by the various methods used for its material characterisation and absorption studies. A direct diode pumped Yb-doped fibre amplified laser system (Clark-MXR Inc. IMPULSE series ultra-short pulse laser) was used to synthesis the materials. Characterisation of morphology was done using SEM, FE-SEM, TEM and HR-TEM. Material and phase characterisation was done using EDX, XRD and XPS. Spectroscopy studies were done using a spectrometer, Raman spectroscope and spectroflurophotometer. 


\section{Chapter 4}

\section{Multiphase Functionalization of Titanium}

Titanium on its own is a highly resistive and reflective metal. Its naturally occurring Oxide, Titanium Dioxide has generated a wide interest due to its strong UV absorbing and photocatalytic properties. It is extensively used as a mesoporous nanoparticle Oxide layer in solar cells and other applications. But is still plagued by the issue of inefficient absorption in the visible and infrared part of the solar spectrum, as indicated earlier in Chapter 1. Despite numerous efforts, there has still not been any substantial gain observed in the vis-NIR region, especially beyond $700 \mathrm{~nm}$. Research in this area is still being pursued and continuous effort is necessary to look for neoteric materials that can effectively tap visible and NIR photons This is to ensure that this part of the solar energy doesn't go untapped and then wasted, usually in the form of heat.

The study in this chapter is an effort directed towards the above-mentioned goal. It aims to improve the photon absorptive capacity of Titanium itself and transform, to a certain extent, its wavelength dependent absorptive response to a wavelength independent one. A novel multiphase Oxide of Titanium was generated by a unique multiphase transformation. This was done to enhance its absorption capability of visible and near infrared region photons and "functionalize" it towards photon absorptive applications. Such a multiphase Oxide of Titanium with a high degree of broadened enhancement in absorption, especially in the NIR region of $800-1000 \mathrm{~nm}$, has seldom been observed earlier for Titanium and its Oxides. 


\subsection{Fabrication of Phase Functionalized Titanium}

The multiphase Titanium Oxide was generated by laser irradiation of pure bulk Titanium by using the laser system described in section 3.1. The grade 2 pure Titanium samples used for the experiments were cut and prepared as per the process given in section 3.2. The experiments were performed at a maximum average power of $16 \mathrm{~W}$.

The laser beam was scanned across the Titanium surface along parallel lines at a constant pulse width of $214 \mathrm{fs}$. The laser fluence was set in the range of $0.8208 \mathrm{~J} / \mathrm{cm}^{2}$ to $2.4614 \mathrm{~J} / \mathrm{cm}^{2}$. Three repetition rates were chosen $8.36 \mathrm{MHz}, 12.54 \mathrm{Mhz}$ and 26.07 $\mathrm{MHz}$. To determine the optimal scanning speed of the laser beam at which the functionalised titanium exhibited maximum absorption, a preliminary set of experiments were conducted. The irradiation process was conducted at laser scanning speeds of $50 \mathrm{~mm} / \mathrm{s}, 100 \mathrm{~mm} / \mathrm{s}$ and $200 \mathrm{~mm} / \mathrm{s}$, where no material removal was observed. The absorption was measured for samples generated at each of these conditions.

With laser pulse duration of $214 \mathrm{fs}$, it was found that the absorbance of the irradiated Titanium surface reached maximum at the scanning speed of $50 \mathrm{~mm} / \mathrm{s}$. Therefore, $50 \mathrm{~mm} / \mathrm{s}$ was chosen as the optimum scanning speed to conduct all the experiments in this study. To test the effect of pulse width on the absorbance, the above-mentioned experiments were repeated at pulse widths of $714 \mathrm{fs}$ and $1428 \mathrm{fs}$. The laser scanning speed was kept constant at the observed optimum value of $50 \mathrm{~mm} / \mathrm{s}$. 


\subsection{Material Characterization Studies}

EDX analysis of the multiphase Titanium Oxide of the laser functionalised Titanium was conducted to quantitatively identify and characterise its principal material components. This study revealed the obvious increase in the Oxygen content in the laser material functionalised zone. Figure 4.1(A) provides the EDX analysis of a laser functionalised Titanium sample done via a line scan, where very little oxygen content along the base Ti substrate area is observed. However, an apparent increase in oxygen content is seen along the area functionalised by the ultra-short laser pulses. This can be confirmed by the greater value of the calculated O/Ti ratio in this area, as seen in the plot of Figure 4.1(C). Further careful study of the O/Ti ratio within the laser functionalised zone showed an increase in the oxygen content on the irradiated lines, bought about by the oxidation process at the ultra-short laser material interaction site. The intensity decreased in between the functionalised lines. This can be seen in Figure 4.1(B).

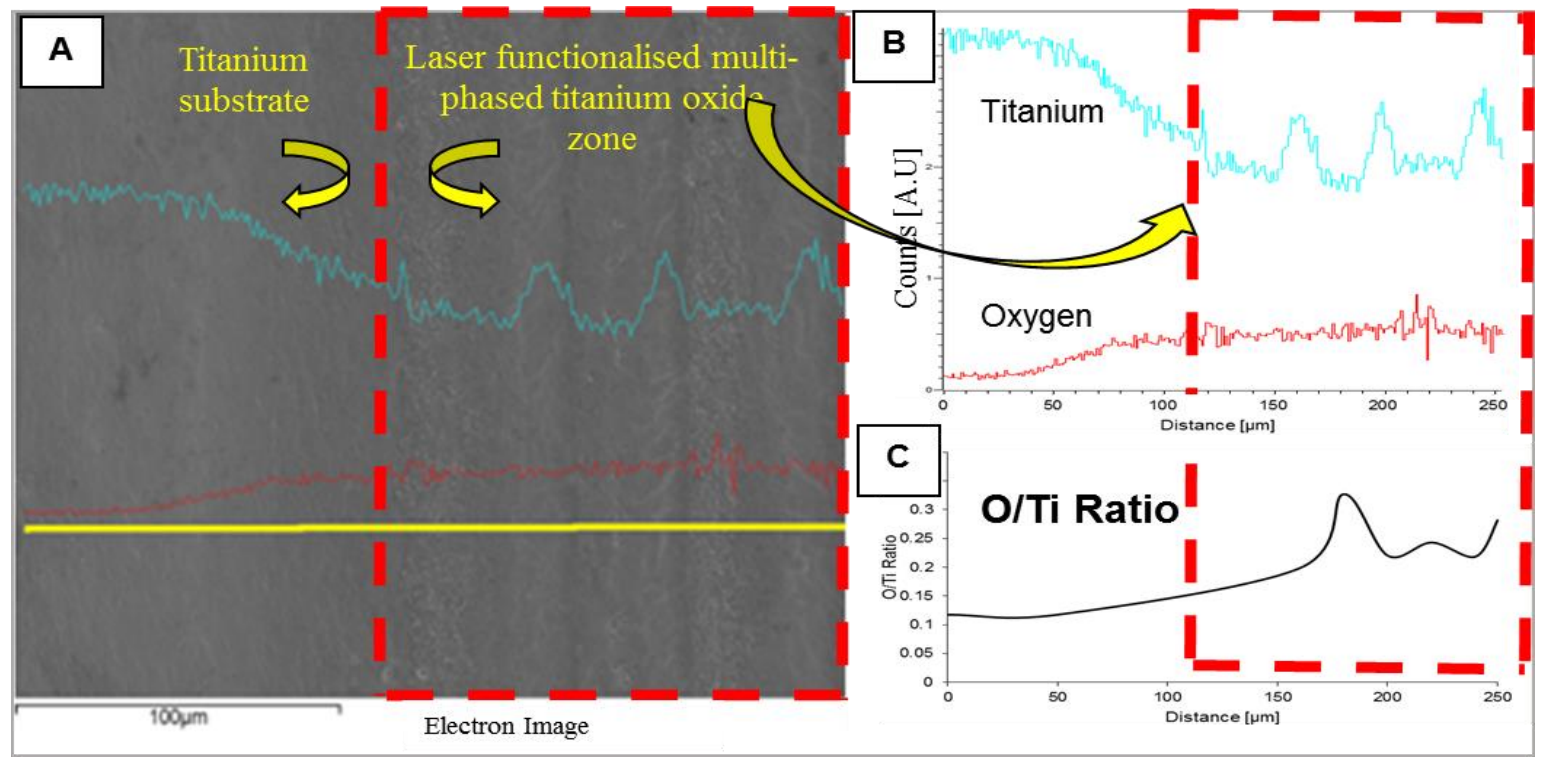

Figure 4.1 (A), (B) EDX line scan showing the variation of Oxygen and Titanium content along the base Titanium substrate and the laser functionalised zone, and (C) O/Ti ratio plot. 
Due to the inability of EDX to help characterise and identify the individual constituent phases of the functionalised Titanium Oxide zone, XRD spectrum was obtained. The resultant XRD pattern revealed that the Oxide layer was composed of three distinct Titanium Oxides phases: (TiO. .716$)_{3.76}$, $\mathrm{TiO}_{2}$ (rutile) and $\mathrm{Ti}_{3} \mathrm{O}$. The basal Titanium substrate showed highly discernible peaks corresponding to Titanium with $\{002\}$ and $\{101\}$ lattice orientations. To quantitatively characterise the multiple phases of the generated Titanium Oxide, Rietveld fitting of the XRD patterns was done.

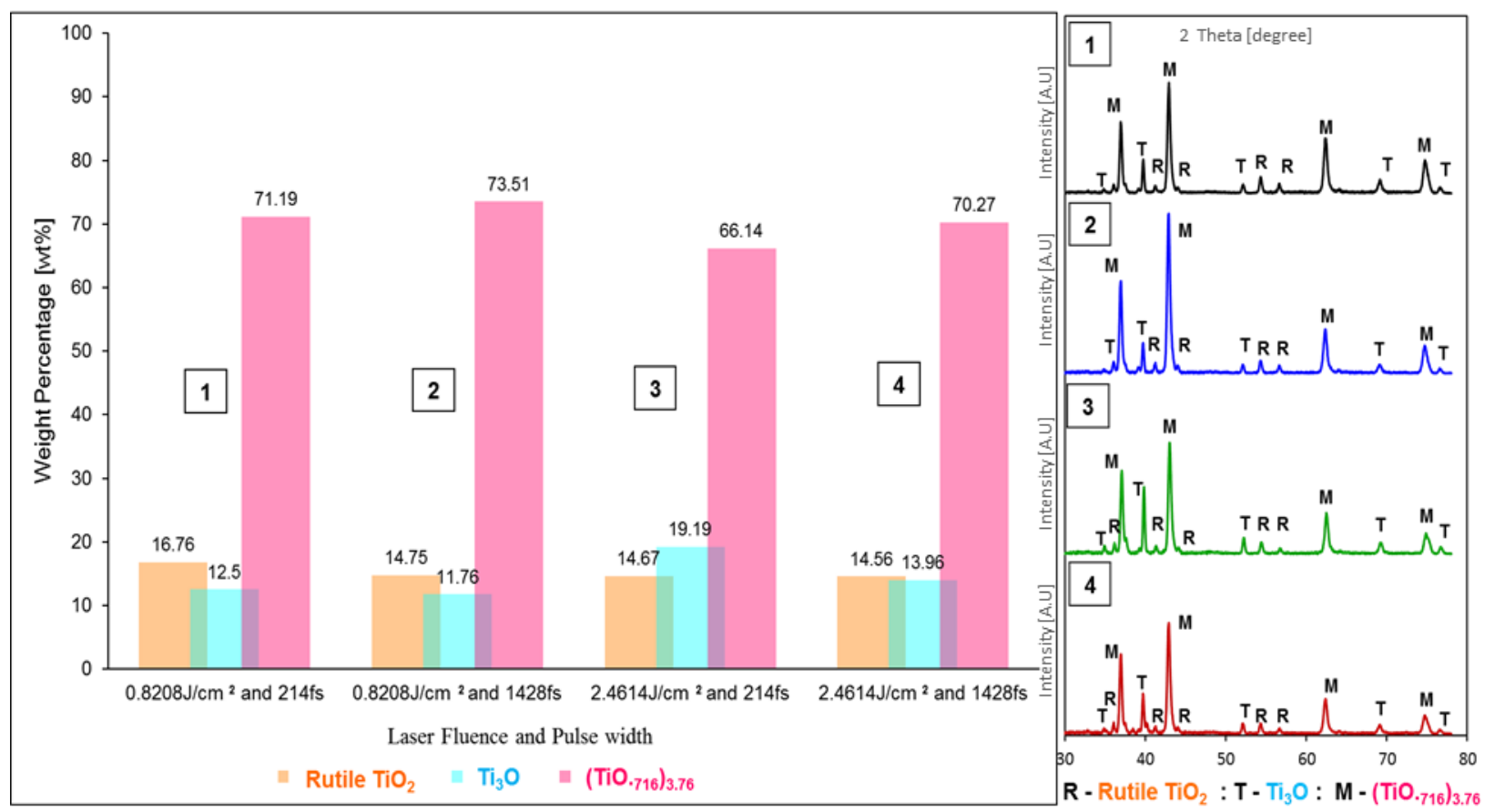

Figure 4.2: wt. \% distribution of each of the three identified Titanium Oxides as obtained by the Rietveld fitting and the corresponding XRD patterns on the right, obtained at various laser parameters.

Figure 4.2 shows the plot indicating weight percentage (wt. \%) of each of the Titanium Oxides formed at a specific laser fluence and pulse width, along with their corresponding XRD patterns obtained at each of those conditions. The most dominant phase ( $70 \%$ weight percentage) was 
found to be a non-stoichiometric Oxide of Titanium having the molecular formula of (TiO.716) 3.76 (PDF2 source pattern: 01-073-1774 and ICSD collection code: 024286). This rare Oxide of Titanium, having an enlarged lattice when compared to that of the base Ti substrate, has a face centered cubic structure (fcc) and a lattice constant of $a=4.1966$ [86]. The structure is similar to defective $\mathrm{NaCl}$ and its atomic position occupancy of $\mathrm{Ti}$ and $\mathrm{O}$ atoms are $94.0 \%$ and $67.3 \%$ respectively [87]. Another rare Oxide of Titanium $\mathrm{Ti}_{3} \mathrm{O}$ (PDF2 source pattern: 01-072-1806 and ICSD collection code: 020041), which has a hexagonal structure with lattice parameters of $a=$ 5.15 and $c=9.56$, accounts for about $15 \%$ of the weight [88]. The remaining is rutile $\mathrm{TiO}_{2}$. It was observed from the Reitveld fitting that at constant pulse width, with a decrease in laser fluence, the percentage composition of ( $\mathrm{TiO} .716)_{3.76}$ and rutile $\mathrm{TiO}_{2}$ increased while that of $\mathrm{Ti}_{3} \mathrm{O}$ decreased. Whereas, at constant laser fluence, as the pulse width decreased, the percentage composition

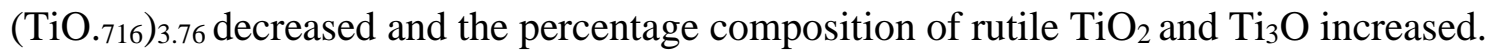

For an X-ray, with a $\mathrm{Cu} \mathrm{K \alpha}$ radiation source, having a constant wavelength of $1.54060 \AA$, the calculated penetration depth is known to be approximately $7-8 \mu \mathrm{m}$ at $2 \theta$ values of $60-65^{\circ}$. The conspicuous absence of the Ti substrate peaks on the XRD plots of the samples shows that the Xrays were not able to penetrate the multi-phased Titanium Oxide layer. It can therefore be safe to ascertain the apparent thickness of the multi-phased Oxide layer on the laser functionalised zones to be at least $7-8 \mu \mathrm{m}$. This absence of $\alpha$-Ti peaks also signified that the femtosecond laser irradiation process lead to a "complete" and not partial oxidation of Titanium. To further corroborate XRD analysis, Raman and XPS spectrum was acquired. The Ti 2p XPS spectrum (given in Figure 4.3(A)) showed $\mathrm{Ti} 2 \mathrm{p}_{3 / 2}$ and $\mathrm{Ti} 2 \mathrm{p}_{1 / 2}$ peaks characteristic of $\mathrm{Ti}^{4+}$ oxidation state and were thus attributed to $\mathrm{TiO}_{2}$. Since XPS is a highly surface sensitive technique, it is possible 
that it could only characterize the top most thin Oxide layer formed upon exposure to the ambient air $\left(\mathrm{TiO}_{2}\right)$ and not the other lower Oxides of Titanium otherwise identified by XRD analysis.
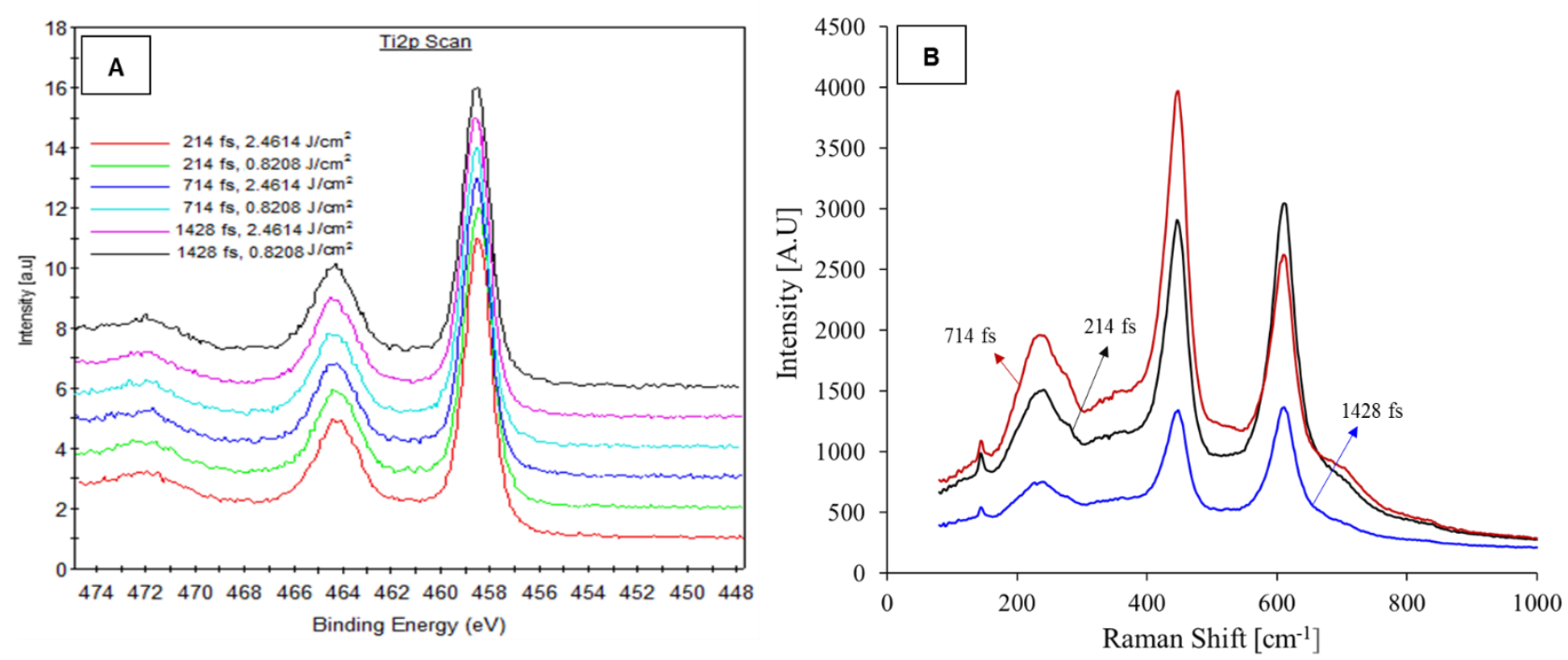

Figure 4.3: (A) Ti 2p XPS spectrum, and (B) Raman spectrum of the laser functionalised zone when synthesised at different laser conditions.

For the Raman spectroscopy analysis, a central wavelength of $532 \mathrm{~nm}$ was used as excitation laser beam. The Raman plot of the non-functionalised Titanium substrate area on samples was devoid of any peaks, which confirmed the absence of Oxides on these areas. The measurements taken at ambient conditions of all the laser functionalised areas on the samples, showed three sharp peaks at the following given wave numbers: $145 \mathrm{~cm}^{-1}, 240 \mathrm{~cm}^{-1}, 443 \mathrm{~cm}^{-1}$ and $610 \mathrm{~cm}^{-1}$. The Raman spectra taken for samples created at $2.4614 \mathrm{~J} / \mathrm{cm}^{2}$ laser fluence and various pulse width values is given Figure 4.3(B). These peaks correspond to the characteristic peaks of rutile $\mathrm{TiO}_{2}$. As already evidenced by the XRD results, no presence of anatase $\mathrm{TiO}_{2}$ was recorded by the Raman spectroscope in all the laser irradiated samples. The absence of the other two Oxides of Titanium 
(TiO.716) $)_{3.76}$ and $\mathrm{Ti}_{3} \mathrm{O}$ on the Raman plots can be explained by the fact that neither of the two Oxides are Raman active due to which they cannot be detected via the Micro Raman.

HR-TEM was used to identify the crystal lattice and verify the presence of the three Titanium Oxide phases identified in the XRD study. The results are given in Figure 4.4. First, the d-spacing of the different lattice orientations were calculated using the Fast Fourier transform (FFT) image obtained by NIH's ImageJTM software. The measured d-spacing values were then compared to those listed in the standard Powder Diffraction File (PDF-2) database to see if the values agreed with any of the three identified Titanium Oxides. In Figure 4.4(A), the d-spacing of the lattice fringes seen in area 1, calculated from the FFT image (inset) was found to be $2.40 \AA$. The two lattice planes seen in the FFT image of area 2 were calculated to have a d-spacing of $3.300 \AA$ and $2.450 \AA$. These values seem to be consistent with the standard PDF2 values of $\{101\}$ orientation plane (i.e. $2.487 \AA$ ) and $\{110\}$ plane (i.e. $3.248 \AA$ ) of rutile phase of $\mathrm{TiO}_{2}$. Furthermore, the tetragonal shape of $\mathrm{TiO}_{2}$ is clearly visible in the marked area 2 .

The lattice d-spacing of the FFT image of the fringes seen in Figure 4.4(B) were calculated to be $3.350 \AA, 2.630 \AA$ and $2.400 \AA$. These d-spacing values are agreeable with the PDF2 values of hexagonal $\mathrm{Ti}_{3} \mathrm{O}$ Oxide. The table in Figure $4.4(\mathrm{C})$ shows the measured d-spacing values and the standard reference values for the corresponding lattice planes of $\operatorname{Ti}_{3} \mathrm{O} .\{102\},\{103\}$ and $\{004\}$ lattice planes with the d-spacing values of $3.260 \AA, 2.592 \AA$ and $2.390 \AA$, respectively. Figure 4.4(D) has three marked areas. The Figure 4.4(E) shows the measured d-spacing values and the standard reference values from the PDF2 source and the Titanium Oxides to which they most correspond to. 

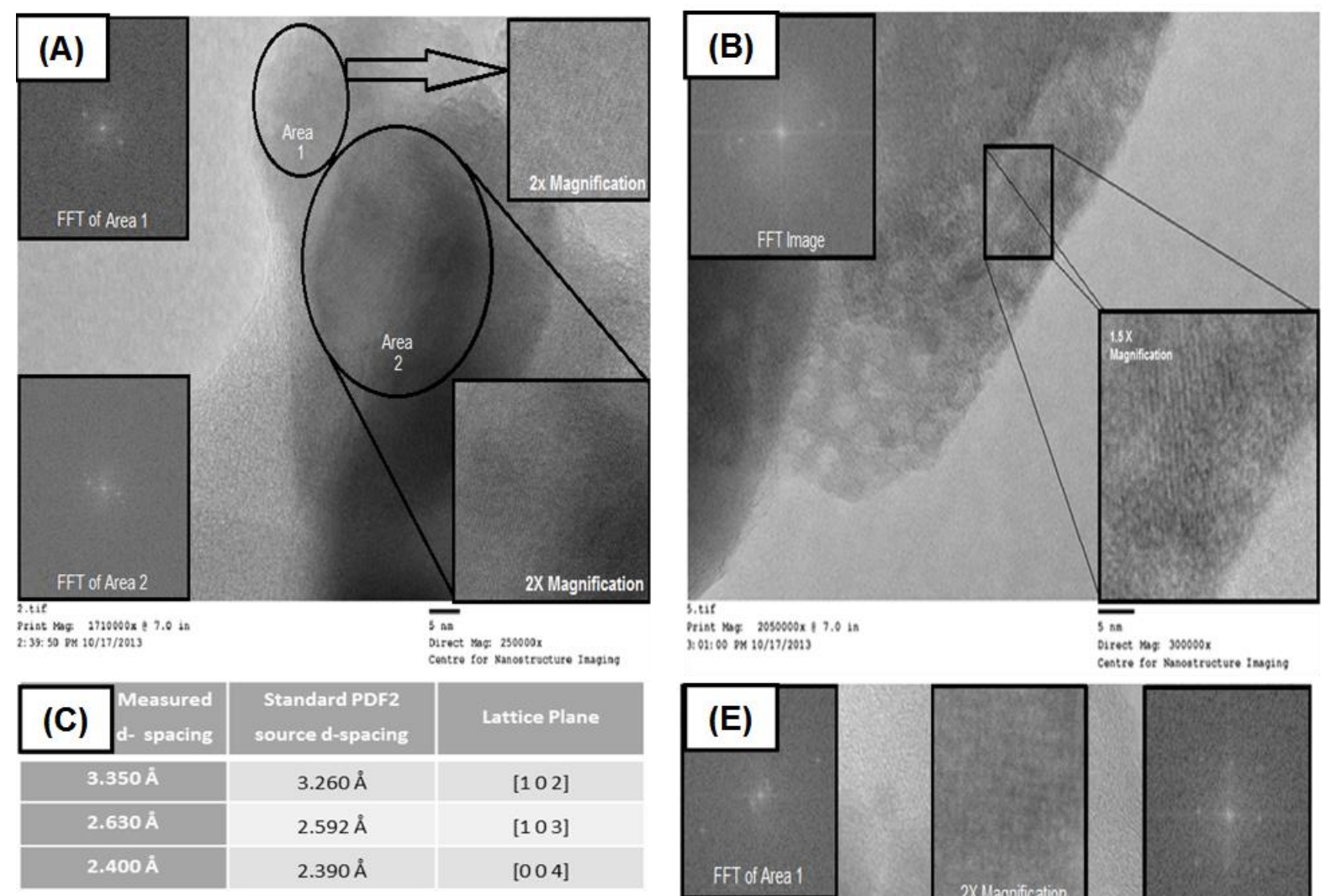

\begin{tabular}{|c|c|c|c|c|}
\hline E) & $\begin{array}{l}\text { Measured } \\
\text { d-spacing }\end{array}$ & $\begin{array}{l}\text { Standard } \\
\text { (PDF2 } \\
\text { source) } \\
\text { d- } \\
\text { spacing }\end{array}$ & $\begin{array}{l}\text { Lattice } \\
\text { Plane }\end{array}$ & $\begin{array}{l}\text { Oxide of } \\
\text { Titanium }\end{array}$ \\
\hline 1 & $2.000 \AA$ & $2.098 \AA$ & {$\left[\begin{array}{lll}2 & 0 & 0\end{array}\right]$} & $\left(\mathrm{TiO}_{716}\right)_{3.76}$ \\
\hline \multirow{4}{*}{2} & $2.300 \AA$ & $2.266 \AA$ & 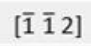 & $\mathrm{Ti}_{3} \mathrm{O}$ \\
\hline & $2.400 \AA$ & $2.390 \AA$ & {$\left[\begin{array}{lll}0 & 0 & 4\end{array}\right]$} & $\mathrm{Ti}_{3} \mathrm{O}$ \\
\hline & $2.500 \AA$ & $2.575 \AA$ & {$\left[\begin{array}{lll}1 & 1 & 0\end{array}\right]$} & $\mathrm{Ti}_{3} \mathrm{O}$ \\
\hline & $3.300 \AA$ & $3.260 \AA$ & {$\left[\begin{array}{lll}1 & 0 & 2\end{array}\right]$} & $\mathrm{Ti}_{3} \mathrm{O}$ \\
\hline 3 & $2.400 \AA$ & $2.422 \AA$ & [ $\left.\begin{array}{lll}1 & 1 & 1\end{array}\right]$ & $\left(\mathrm{TiO}_{.716}\right)_{3.76}$ \\
\hline
\end{tabular}

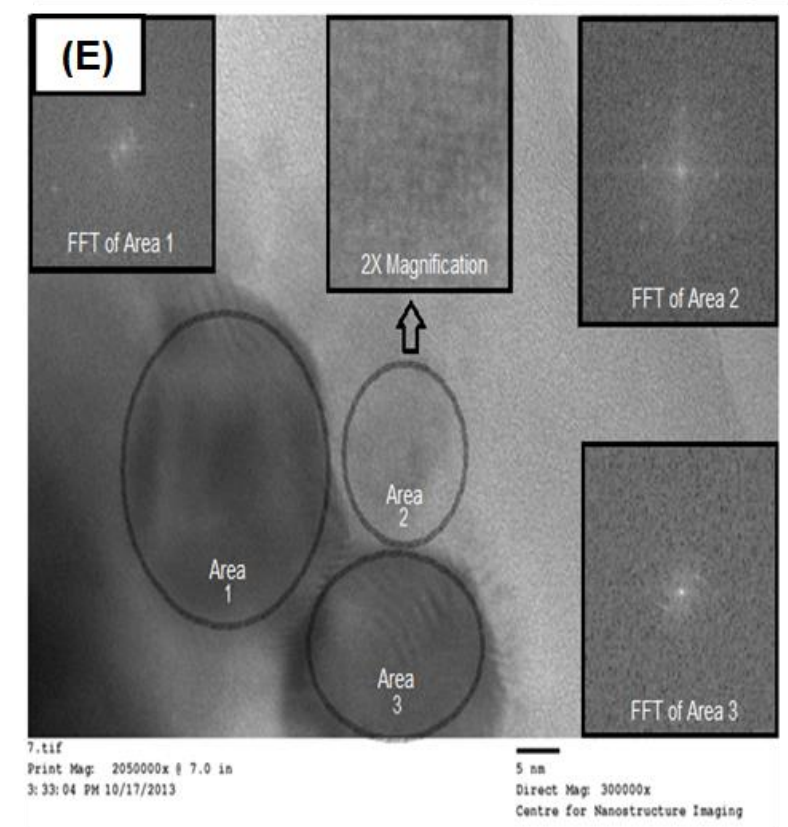

Figure 4.4: HRTEM image of (A) rutile $\mathrm{TiO}_{2}$ particle showing different planes in area 1 and 2, (B) hexagonal $\mathrm{Ti}_{3} \mathrm{O}$, with the FFT and magnified image given in the inset, (C) d-spacing values of different planes of $\operatorname{Ti}_{3} \mathrm{O}$ as measured from figure (B) and standard (PDF2 source) values, (D) fcc-structured ( $\left.\mathrm{TiO}_{.716}\right)_{3.76}($ area 1 and 3$)$ and $\mathrm{Ti}_{3} \mathrm{O}(\operatorname{area} 2)$, with the FFT image given in the inset, and (E) Measured and standard (PDF2 source) d-spacing values of fringes present in different marked areas of figure and the corresponding identified Oxide of Titanium. 


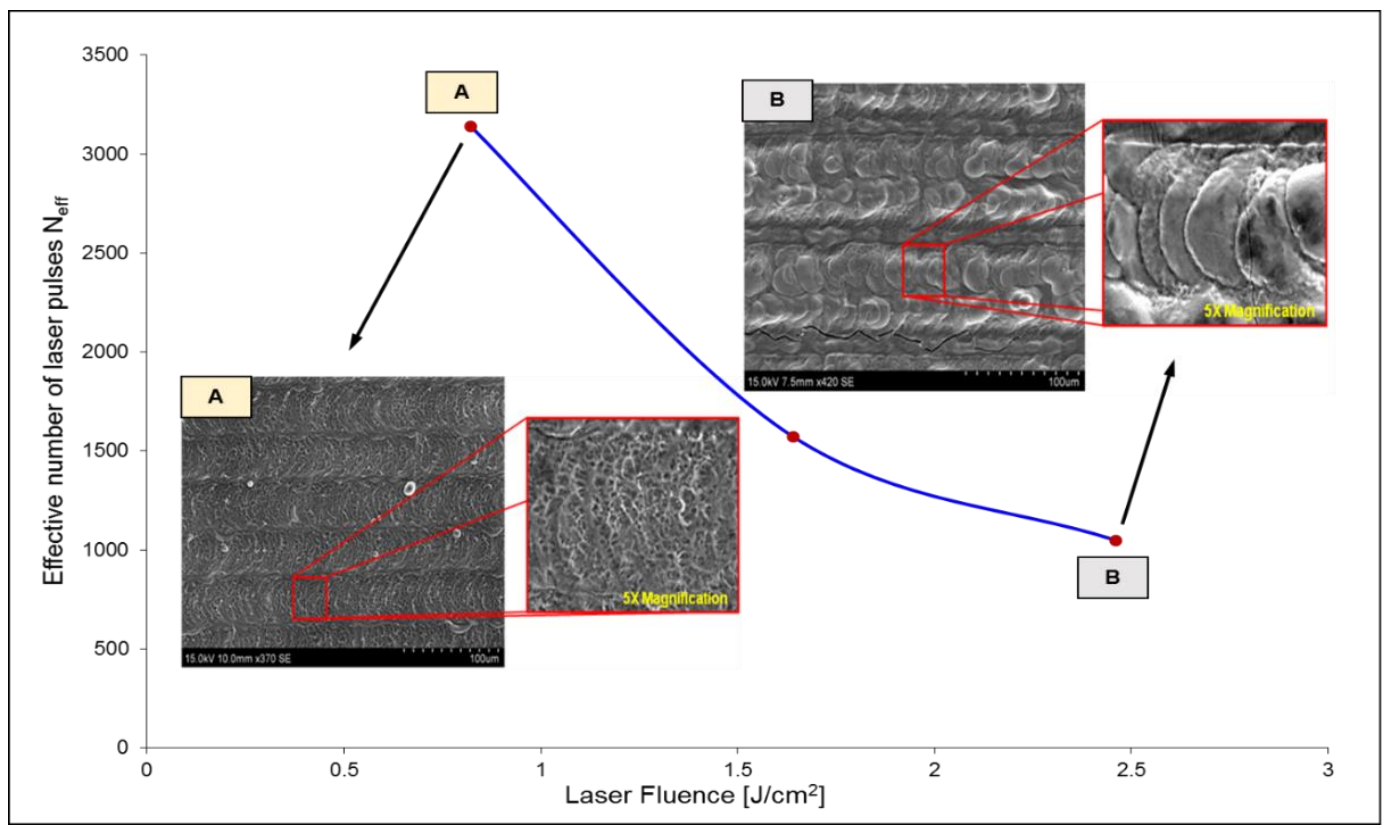

Figure 4.5: $N_{\text {eff }}$ vs. Laser fluence plot with the corresponding SEM images of the laser functionalised zone at extreme condition of laser fluence.

Surface morphology of the laser functionalised samples was then examined via SEM to reveal the presence of a molten recast layer. This molten recast was more pronounced at higher laser fluence values and was seen to have 'round platelet' like formation. The overlapping of the platelets was seen to reduce with a decrease in the effective number of pulses due to fewer number of laser pulses hitting the same spot on the metal target before the laser moves on to the adjacent area. On the other hand, a 'web-like' surface was observed for areas functionalised at lower laser fluence. No removal of material was observed on any of the laser transformed samples. Figure 4.5 shows the variation of number of ultra-short laser pulses hitting the Titanium sample surface as the fluence is changed. The corresponding change in surface morphology seen is indicated by the SEM images provided in the inset. 


\subsection{Photon Absorption Under Various Synthesis}

\section{Conditions}

The absorption properties of the irradiated Ti samples were obtained using the spectrometer. The absorption was then plotted against the wavelength, at constant laser fluence values. The absorption properties of the multi-phased Titanium Oxide zone were then compared with that of the base untreated Ti substrate.

Several interesting observations were made. The absorption intensity showed a steady increase as the wavelength increased from $300 \mathrm{~nm}$ to $1000 \mathrm{~nm}$. The absorption plateaued for wavelengths greater than $750 \mathrm{~nm}$. This signified that the laser irradiation process had a significant impact on the absorbance properties of the phase transformed Titanium in the vis-NIR region of the solar spectrum. At a given laser pulse width, the direct proportionality of absorbance with the effective number of pulses and inverse variation with the laser fluence was observed.

The spectral studies performed for samples prepared at different pulse widths revealed a similar increase in absorbance at greater wavelengths with respect to the base non-laser transformed Titanium surface. This can be seen in Figure 4.6. It was observed at higher laser fluence that the absorbance tended to increase as laser pulse width became longer (Figure 4.6(A)). However, this increase in absorbance with pulse width showed a reverse trend from 714 fs to 1428 fs at a lower laser fluence values, as seen in Figure 4.6(B) and (C). This might indicate the presence of a lower threshold value of laser fluence beyond which, the effect of increase in pulse width does not result in absorption enhancement but rather veritably, leads to its inhibition. 

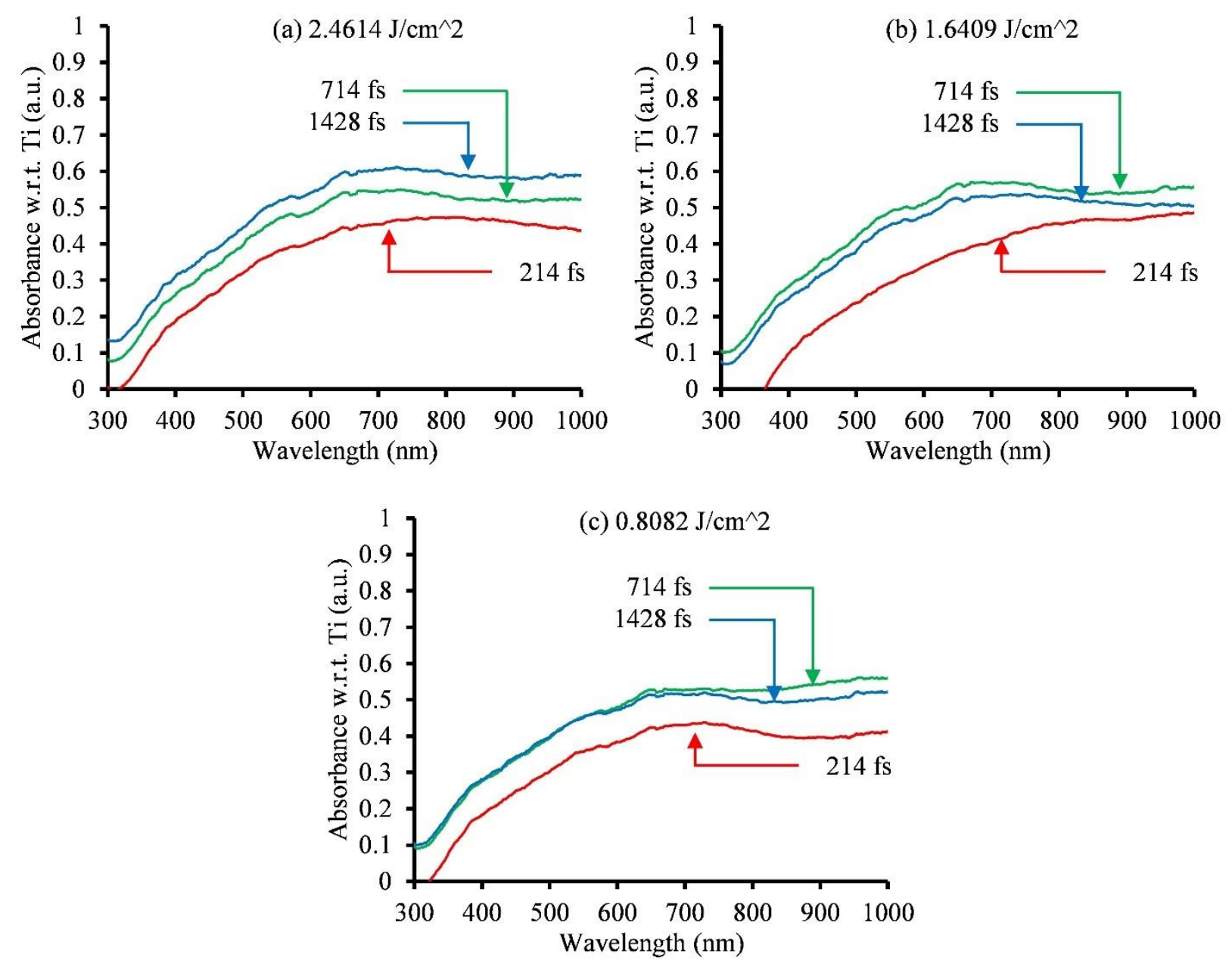

Figure 4.6: Absorbance measured at different wavelengths for the three pulse width values of $214 \mathrm{fs}$ (red), $714 \mathrm{fs}$

(green), $1428 \mathrm{fs}$ (blue), at a constant laser fluence value of (A) $2.4614 \mathrm{~J} / \mathrm{cm}^{2}$, (B) $1.6409 \mathrm{~J} / \mathrm{cm}^{2}$, and (C) $0.8082 \mathrm{~J} / \mathrm{cm}^{2}$.

Further comparative analysis was done by plotting the increment in absorbance against the Reitveld fitted weight percentage values of each of the Titanium Oxides calculated earlier for each laser parameter. It was observed that the variation in the weight percentage of each of the three Oxides had a much greater influence in the absorption at wavelengths longer than $800 \mathrm{~nm}$. It was also observed that at longer pulse durations, where lower amounts of rutile and more of (TiO.716)3.76 were present, a considerable amount of gain in absorption, in the wavelength range of 800-1000 
$\mathrm{nm}$ was seen. It is thus possible that the non-stoichiometric Oxide phase of Titanium (TiO.716) 3.76 might have played a role in such an enhancement of absorption in the NIR region. This is elucidated in Chapter 7.

\subsection{Band Gap Calculations}

To further study the optical properties of the multi-phased Oxide of Titanium, its band gap, $E_{\mathrm{g}}$, was calculated from the optical absorbance spectrum, $\varepsilon(\lambda)$. The Tauc's equation was used to measure the band gap [89]:

$$
\omega^{2} \varepsilon=\left(h \omega-E_{\mathrm{g}}\right)^{2}
$$

where $\omega=2 \pi / \lambda$, and $h$ is the Plank's constant. The intersection of a straight line extrapolated from

the curve $\varepsilon^{\frac{1}{2}} / \lambda$ vs. $1 / \lambda$ with the abscissa axis gives the value of the band gap. The units of abscissa axis are formatted to give the energy value $E$, in electron volts $(E=h c / \lambda)$, where $c$ is the speed of light. The table 4.1 gives the band gap values calculated for the functionalised Titanium generated at various laser parameters. It was observed from the above calculation that the multiphased Titanium Oxide exhibited an average band gap value of $2.39 \mathrm{eV}$, which is much lower than the bulk $\mathrm{TiO}_{2}$ band gap value of $3.2 \mathrm{eV}$. The presence of the (TiO.716) 3.76 in the multi-phased laser functionalised zone, could have rendered the lowering of the band gap value by the virtue of its non-stoichiometry [90], [66].

\begin{tabular}{lccc}
\hline \hline & \multicolumn{3}{c}{ Laser scanning speed $(\mathrm{mm} / \mathrm{s})$} \\
\cline { 2 - 4 } & 200 & 100 & 50 \\
\cline { 2 - 4 } Laser fluence $\left(\mathrm{J} / \mathrm{cm}^{2}\right)$ & $E_{\mathrm{g}}(\mathrm{eV})$ & $E_{\mathrm{g}}(\mathrm{eV})$ & $E_{\mathrm{g}}(\mathrm{eV})$ \\
\hline 2.4614 & 2.6 & 2.6 & 2.4
\end{tabular}


1.6409

0.8208

Table 4.1: Band gap values, Eg (using Tauc's equation), of the functionalised Ti generated at various laser parameters.

\subsection{Summary}

In this chapter, a novel Oxide of Titanium composed of multiple rare phases is reported, which due to its notable vis-NIR absorption capabilities has the potential viability of being used as an intrinsic photon absorber. The multi-phased Titanium Oxide, composed of three different Oxide phases of Titanium, was developed by a unique interaction between ultra-short laser pulses and the base Ti substrate. The composition of each of these phases was shown to vary with the ultrafast laser processing parameters, which induced photon absorption properties to the multi-phased Titanium Oxide material. The fabrication of this multi-phased Oxide of Titanium yielded to the transformative phase functionalization of the base Ti towards an average of almost a three-fold absorption gain in the visible region and a four-fold absorption gain in the NIR region of the solar spectrum. Characterization studies were performed to identify the discrete Titanium Oxides. This was followed by a transient temperature analysis, given in appendix A, to understand the ultrafast laser processing conditions that were conducive for the formation of each of Oxide phase. The optical band gap of the generated rare multiphase Oxide of Titanium was averaged to be around $2.39 \mathrm{eV}$, which is about $25 \%$ lower than that rutile and $20 \%$ lower than anatase, the two most prevalent phases of $\mathrm{TiO}_{2}$. A window of opportunity exists to capitulate on the high photon absorptive properties of the generated multi-phased Oxide of Titanium and thereby transform and fine tune Titanium into a highly photon sensitive material in the vis-NIR region. The significant 
absorption gain thus reported, especially in the NIR of the solar spectrum, by the unique phase functionalization of Titanium can be availed in the continued pursuit over a "full spectrum" photon absorption. 


\section{Chapter 5}

\section{Synthesis of Multiphase Nano $\mathrm{TiO}_{\mathrm{x}}$}

\section{Networks}

In this chapter, a photo absorptive nanomaterial network synthesised by incorporating the multiphase Titanium Oxide presented in Chapter 4 is illustrated. The multiphase aspect of the Titanium Oxide nanomaterial has been exploited to expand the absorption range and demonstrate greater sensitivity towards photons of wider area of the vis-NIR region. This nanomaterial is structurally $3 \mathrm{D}$ in nature and dopant free. It has been synthesised by the formation of an ionised plume and a unique phase transformation process brought about by the interaction of high energy ultra-short pulses with the Titanium metal substrate. It has exhibited enhanced absorption that is three times greater in visible region and six times greater in NIR than base Titanium. The synthesis of such a highly photo absorptive nano network serves as an important first step towards improving the prospect of it being implemented in various photon harvesting devices.

\subsection{Self-Assembly of Nano $\mathrm{TiO}_{\mathrm{x}}$ Network}

The Titanium Oxide nanomaterial was generated under conditions of standard ambient temperature and pressure (SATP) and at laser fluence values of $5.14 \mathrm{~J} / \mathrm{cm}^{2}, 2.46 \mathrm{~J} / \mathrm{cm}^{2}$ and $1.64 \mathrm{~J} / \mathrm{cm}^{2}$ (which correspond to repetition rates of $4 \mathrm{MHz}, 8.36 \mathrm{Mhz}$ and $12.54 \mathrm{MHz}$ 
respectively), each at three different pulse widths (durations): $214 \mathrm{fs}, 714 \mathrm{fs}, 1428 \mathrm{fs}$, with peak power ranging from $90 \mathrm{MW} /$ pulse to $1885 \mathrm{MW} /$ pulse. The laser beam was scanned normally on the sample surface at the rate of $5 \mathrm{~mm} / \mathrm{s}$ with an average power of $16 \mathrm{~W}$. Titanium samples were cut and prepared as described in section 3.2.

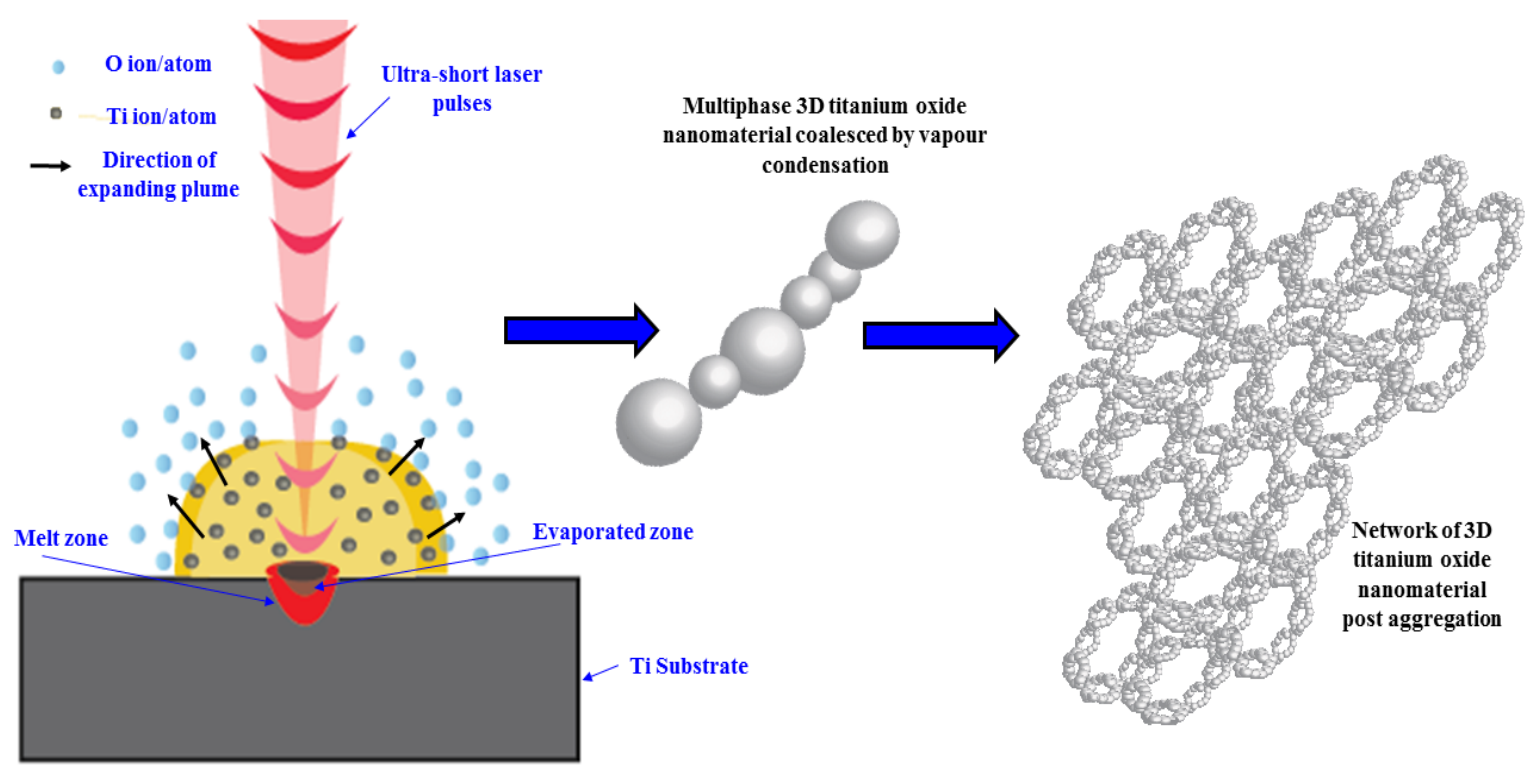

Figure 5.1: Multiphase Titanium Oxide nanomaterial formed by ultra-short laser synthesis mechanism in ambient air.

As elucidated in Chapter 2, the synthesis of the Titanium Oxide nanomaterial via ultra-short laser material interaction can occur either due to the initial ejection of target Titanium atoms in a coalesced form or by the subsequent collisions and assembling of ablated atoms in the plume. The interaction time between the ultra-short laser pulses (femtosecond (fs) duration) and the Titanium target material is extremely brief in nature. Due to this, it is known that the bulk of the target lattice remains cold and only electron excitation takes place, causing the density of the target material to remain constant [91]. The multiple ultra-short laser pulses hit the target with a time separation of anywhere between 40 to 250 nanoseconds between each consecutive pulse (depending on the 
repetition rate chosen). This results in a large amount of energy being transferred to the target material (Titanium) but not enough time for it to be dissipated before the next pulse hits the sample. This leads to the formation of a plasma plume consisting mostly of ejected Titanium target material atomic species which subsequently diffuse and collide with the air molecules. The collision of the target Titanium species with the air molecules leads to their recombination and reduction in temperature. Vapour condensation then follows, leading to the nucleation and growth of the coalesced nanomaterial and the subsequent formation of a three-dimensional network due to aggregation. This laser synthesis mechanism has been illustrated in Figure 5.1.

The number of ultra-short pulses interacting with the Titanium surface in a unit time and the temperatures generated on the sample surface and in the plume of atomic species, are important parameters which influence the synthesis of the nanomaterial.

\subsection{Surface and Material Phase Characterization}

Prior to identifying the material and phase composition of the synthesised nanomaterial, its surface morphology was inspected using an SEM. The study revealed the presence of a three-dimensional network of nanomaterial composed of individual spherical nanostructures agglomerated and coalesced together. The density of this nano network was seen to diminish with the lowering of the laser fluence as well as the peak power at which they were generated. The SEM images given as an inset in Figure 5.2(C) show the areas scanned by the ultra-short laser pulses, are completely camouflaged by a dense network of nanomaterial at $5.14 \mathrm{~J} / \mathrm{cm}^{2}$ laser fluence. As the fluence reduced to $2.46 \mathrm{~J} / \mathrm{cm}^{2}$, gradual appearance of the contours of the parallel lines along which the laser beam was scanned is seen (Figure 5.2(B)). At the lowest value of laser fluence, $1.64 \mathrm{~J} / \mathrm{cm}^{2}$, a stark pattern of the laser irradiated lines was observed due to a decrease in the density of the 
synthesised nanomaterial. Few aggregated nanostructures were seen extending and meshing over the grooves present in between two scanned lines (Figure 5.2(A)). Additionally, the presence of aggregated 3D nanostructures that further coalesced together to form circular "pores" was also seen, the size of which was observed to diminish with laser fluence [92].
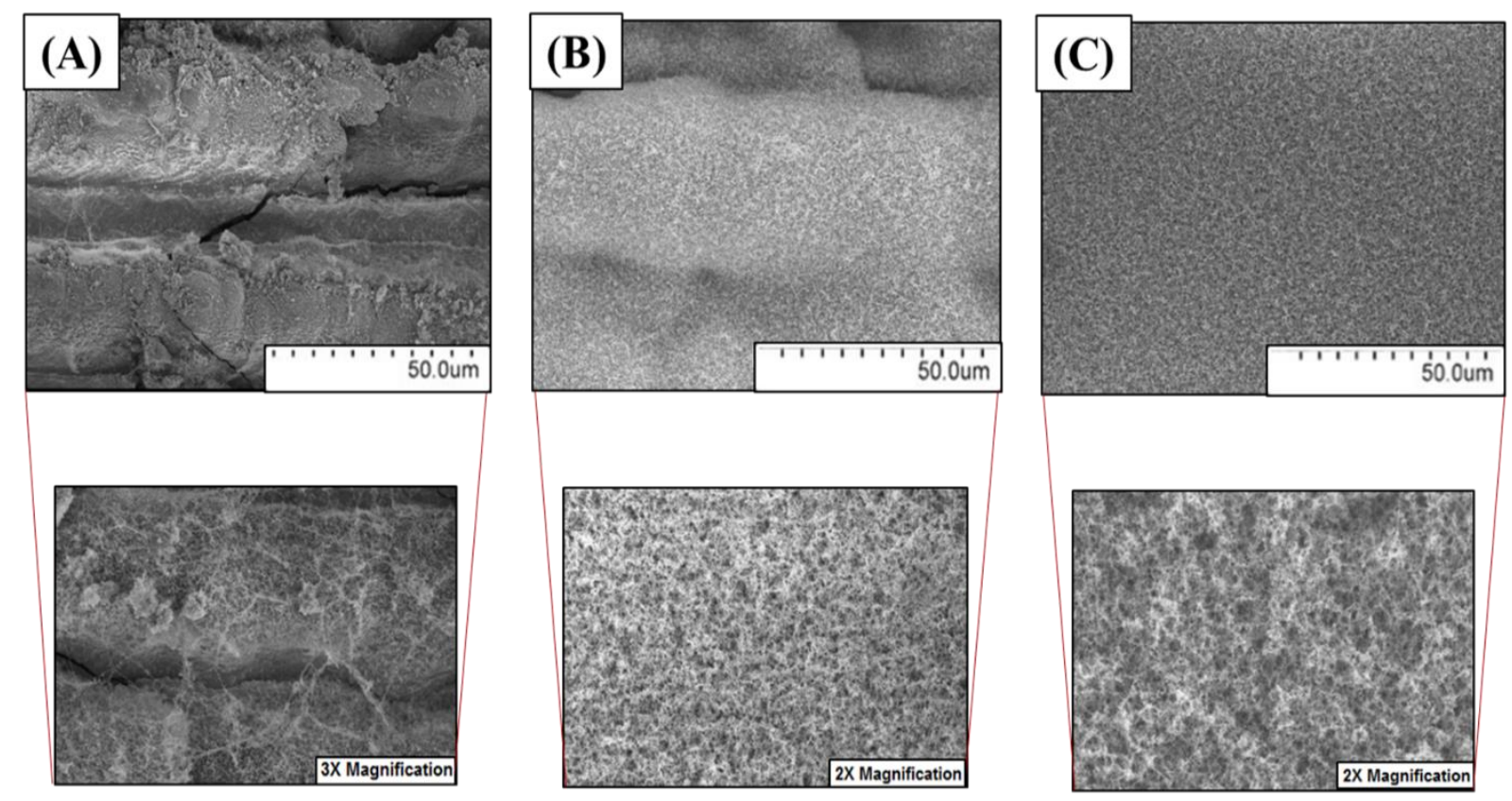

Figure 5.2: SEM images of the nanomaterial network generated at (A) $1.64 \mathrm{~J} / \mathrm{cm}^{2}$, (B) $2.46 \mathrm{~J} / \mathrm{cm}^{2}$, and (C) $5.14 \mathrm{~J} / \mathrm{cm}^{2}$ laser fluence values.

To determine the size distribution of the individual nanospherical structures which agglomerated together to form the aggregated 3D nano network, TEM analysis of the nanomaterial was conducted. Figure 5.3 shows the particle count distributions at different laser processing conditions, with the TEM micrographs of nanospheres given in the inset. It was observed that for a given laser pulse width duration, as the laser fluence was increased, the mean size of the synthesised nanostructures also increased. Figure 5.3(B) and Figure 5.3(A) show this increase in 
the mean size of the synthesised nanospheres as the laser fluence value increased from $1.64 \mathrm{~J} / \mathrm{cm}^{2}$ to $5.14 \mathrm{~J} / \mathrm{cm}^{2}$, at a constant pulse duration of $214 \mathrm{fs}$.
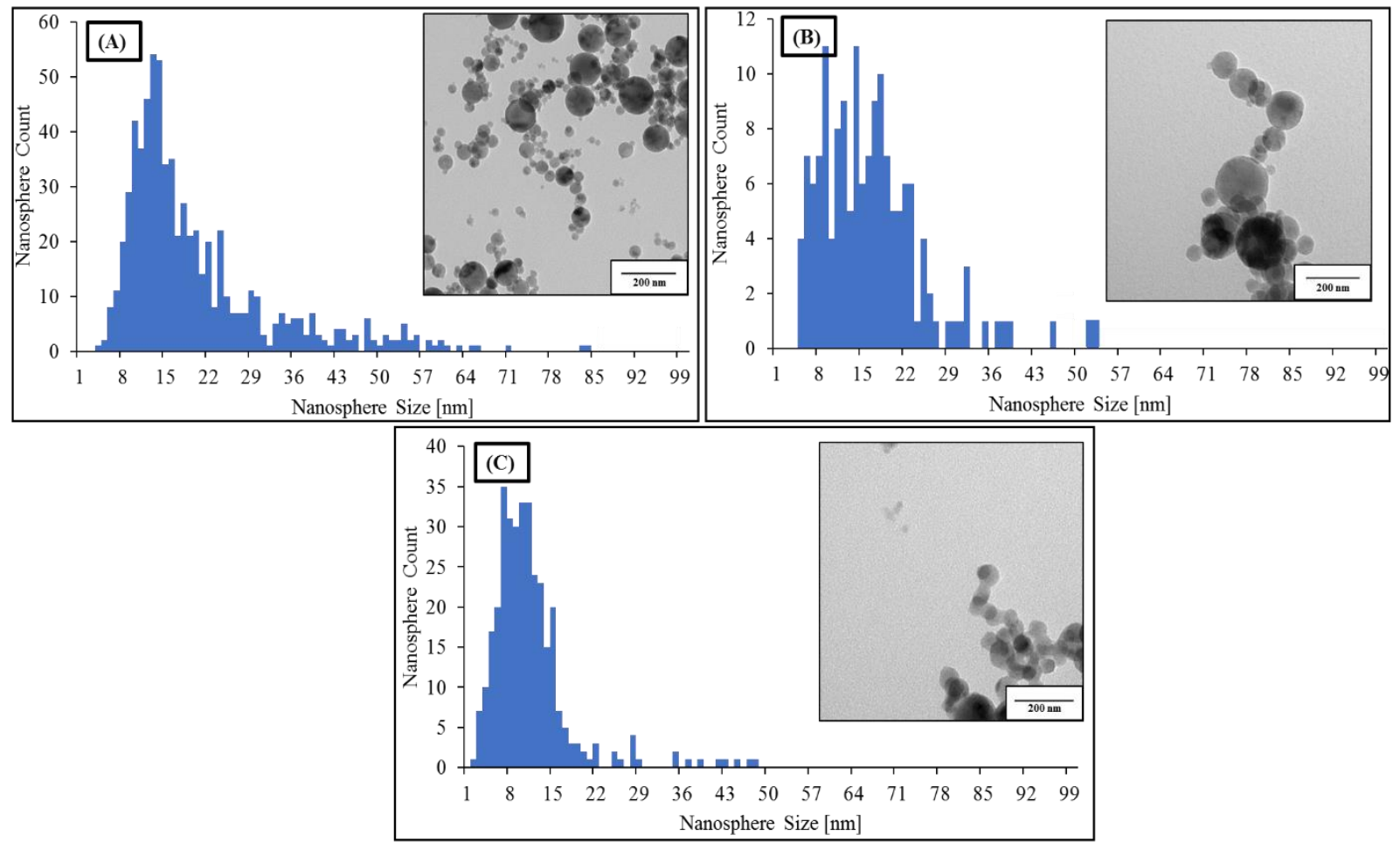

Figure 5.3: Size distribution of the nanospheres of the nano network synthesised at laser fluence and pulse width values of (A) $5.14 \mathrm{~J} / \mathrm{cm}^{2}, 214 \mathrm{fs}$, (B) $1.64 \mathrm{~J} / \mathrm{cm}^{2}, 214 \mathrm{fs}$, and (C) $5.14 \mathrm{~J} / \mathrm{cm}^{2}, 1428 \mathrm{fs}$ calculated using TEM micrographs. Inset shows a TEM micrograph of the nanostructures formed at the given laser condition.

It was observed that at $5.14 \mathrm{~J} / \mathrm{cm}^{2}$, the presence of nanospheres larger than $20 \mathrm{~nm}$ in size increased. Furthermore, at a given laser fluence value, larger nanospheres were observed at shorter pulse widths. This is indicated in Figure 5.3 where, at a constant laser fluence of $5.14 \mathrm{~J} / \mathrm{cm}^{2}$, the mean size of the nanospheres decreases from $13 \mathrm{~nm}$ at a pulse width of 214 fs to $10 \mathrm{~nm}$ at $1428 \mathrm{fs}$ pulse width. The visible increase in the density of the network of nanomaterial formed at higher laser fluence, as well as the corresponding increase in the average size of the nanospheres can be attributed to two factors. One, the high surface and plume temperatures generated because of 
greater laser fluence. Two, the reduction in the number of ultra-short pulses interacting with the sample Titanium surface (but with greater peak power per pulse). Reduction in number of pulses hitting the sample causes an attrition in the number of substrate atoms evaporated by the incident laser beam. This results in fewer number of possible nanoparticle nucleation sites in the plume, which inevitably lead to an enhancement in the particle size [93]. Also, the existence of such high temperatures at higher fluences and shorter pulse width conditions results in the arrival of the next pulse to occur much before the temperature has cooled below the vaporisation point. As such, greater coalescing and agglomeration of the spherical nanostructures takes place, with larger nanostructures being formed. Furthermore, a narrower nanosphere size distribution was observed at longer pulse widths (Figure 5.3(C)) than at shorter pulse durations (Figure 5.3(A) \& Figure 5.3(B)). The presence of a bimodal size distribution was observed at the higher laser fluence value of $5.14 \mathrm{~J} / \mathrm{cm}^{2}$. This can be attributed to an integrated effect on the particle growth rate at such higher fluence values, by both the ionised as well as neutral species in the plume generated [94].

The elemental and phase composition of the synthesised nanonetwork was then determined. EDX analysis of high resolution SEM images of nanospheres revealed their main constituents to be Titanium and Oxygen. Figure 5.4(A) shows the electron image of the aggregated nanospheres synthesised at a laser fluence of $5.14 \mathrm{~J} / \mathrm{cm}^{2}$ and a pulse width of $1428 \mathrm{fs}$. Its Oxygen and Titanium distribution obtained by the EDX area scan analysis, is shown in Figure 5.4(B) and Figure 5.4(C) respectively. This indicated that the nanomaterial was composed of an Oxide of Titanium. Figure 5.4(D) shows the EDX line scan of a single nanosphere formed at a laser fluence of $1.64 \mathrm{~J} / \mathrm{cm}^{2}$ and pulse width of 214 fs. 

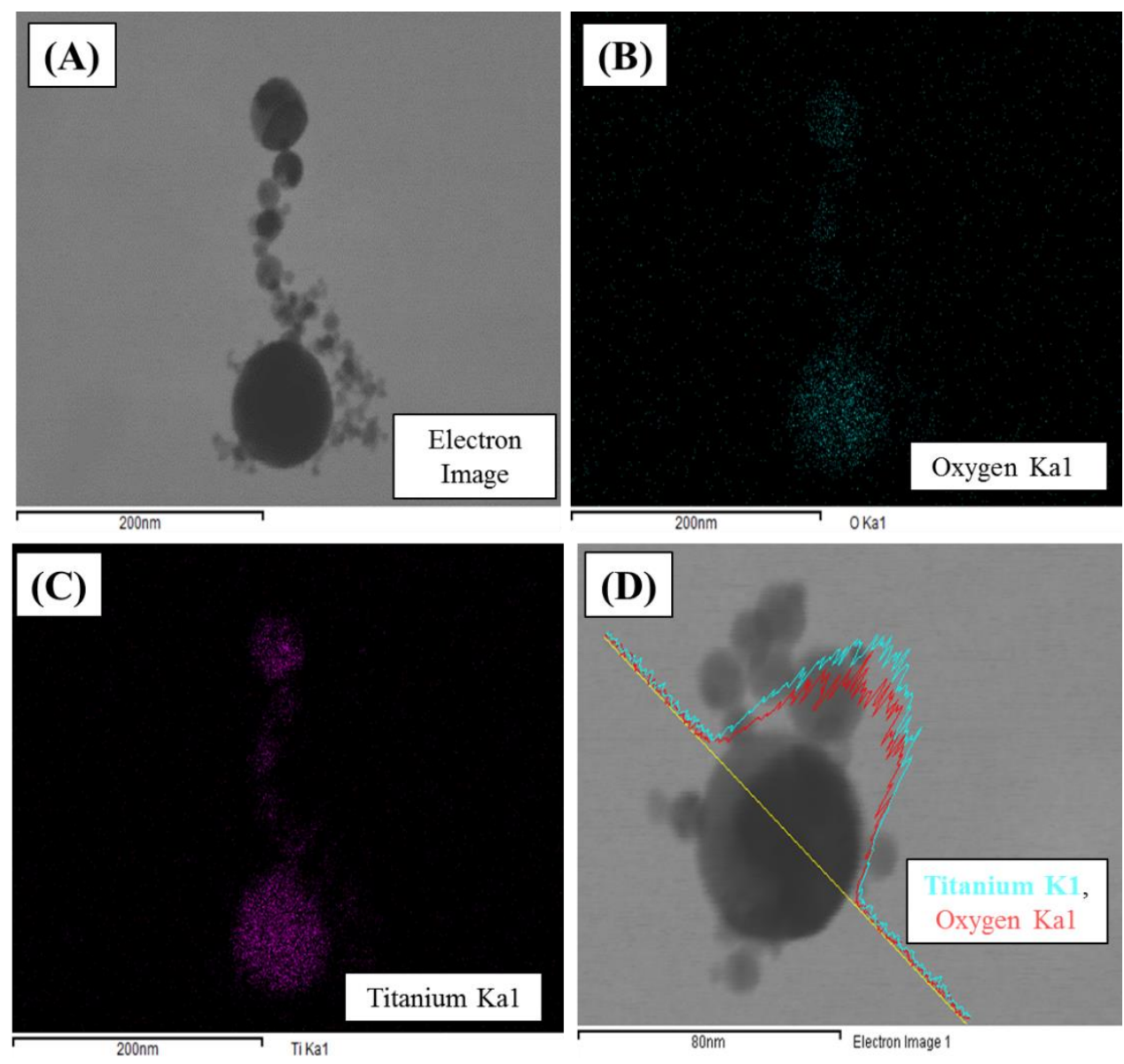

Figure 5.4: (A) Electron image of nanonetwork captured by HR-SEM and the corresponding EDX area scan showing its (B) Oxygen, and (C) Titanium elemental distribution. (D) EDX Line scan analysis of a single spherical nanostructure.

XPS spectral analysis was conducted to yield further information on the composition and the chemical state of the synthesised nanomaterial. The XPS spectrum of core level Ti $2 p$ showed peaks corresponding to $\mathrm{Ti} 2 \mathrm{p}_{3 / 2}$ and $\mathrm{Ti} 2 \mathrm{p}_{1 / 2}$. The binding energy of Ti $2 \mathrm{p}_{1 / 2}$ varied in the range of $464.7 \mathrm{eV}$ to $465 \mathrm{eV}$, whereas the binding energy of Ti $2 \mathrm{p}_{3 / 2}$ varied from $458.9 \mathrm{eV}$ to $459.4 \mathrm{eV}$. These correspond well to the binding energies reported for $\mathrm{TiO}_{2}$ [95]. Thus, the nanomaterial was considered to be composed of Titanium Dioxide phases. Further investigation of the XPS spectrum showed a chemical shift of about $0.4 \mathrm{eV}$ of the Ti $2 \mathrm{p}_{3 / 2}$ core line, towards lower binding energies 
at higher fluence and shorter pulse widths. This indicated that the nanomaterial synthesised under these conditions could have lower oxidation states of titanium and the phase of $\mathrm{TiO}_{2}$ present could be rutile (Figure 5.5) [95]. The phase of $\mathrm{TiO}_{2}$ identified at lower fluences and longer pulse widths was anatase (Figure 5.5) [95].

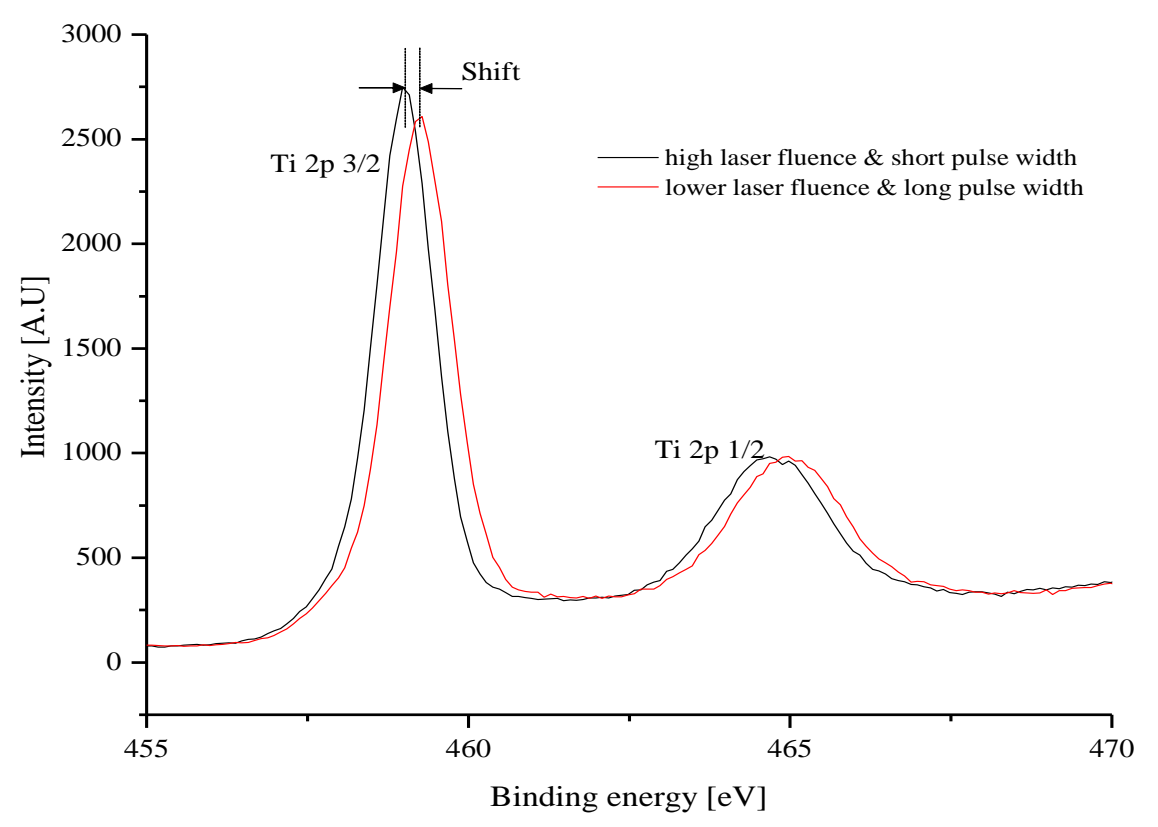

Figure 5.5: XPS spectrum showing a chemical shift for the nanomaterial synthesised at different lasing conditions.

Due to the highly surface sensitive nature of the technique involved in XPS study, XRD analysis was conducted to confirm the above observations and further characterize the phase composition of the nanomaterial. The XRD patterns thus obtained showed the presence of additional phases of Titanium Oxide and exhibited the synthesised nanomaterial to be uniquely 'multiphase' in nature. For the nanomaterial synthesised at the highest fluence value of $5.14 \mathrm{~J} / \mathrm{cm}^{2}$ (Figure 5.6(A)), the XRD pattern had peaks corresponding to four different Oxide phases of Titanium: (1) anatase and rutile, the two most dominant phases of $\mathrm{TiO}_{2}$, (2) $\mathrm{Ti}_{3} \mathrm{O}$, a rare low temperature Oxide of Titanium, (3) (TiO.716) 3.76, a non-stoichiometric Monoxide of Titanium. However, for the nanomaterial 
formed at the lowest laser fluence value of $1.64 \mathrm{~J} / \mathrm{cm}^{2}$ (Figure 5.6(B)), anatase and rutile were the only phases present. As such, at highest laser fluence values, which also corresponded to the lowest frequency of ultra-short pulses interacting with the Titanium sample surface, a greater variation in the degree of phase transformation process was observed in the synthesis of the nanomaterial. Here, both higher and lower Oxides of Titanium were present. Due to the extreme high temperatures being generated within a very short time span at such laser conditions, rapid cooling follows post the cessation of laser-material interaction. Such an environment can serve as a priori for vacancies to be present in the synthesis of nanostructures resulting in the formation of nonstoichiometric Oxides of Titanium, which could explain the presence of $(\mathrm{TiO} .716)_{3.76}$ in the multiphase nanomaterial. Due to the multiphase nature of the nanonetwork formed, for clarity the nanomaterial will be addressed as multiphase 'nano $\mathrm{TiO}_{\mathrm{x}}$ network'.
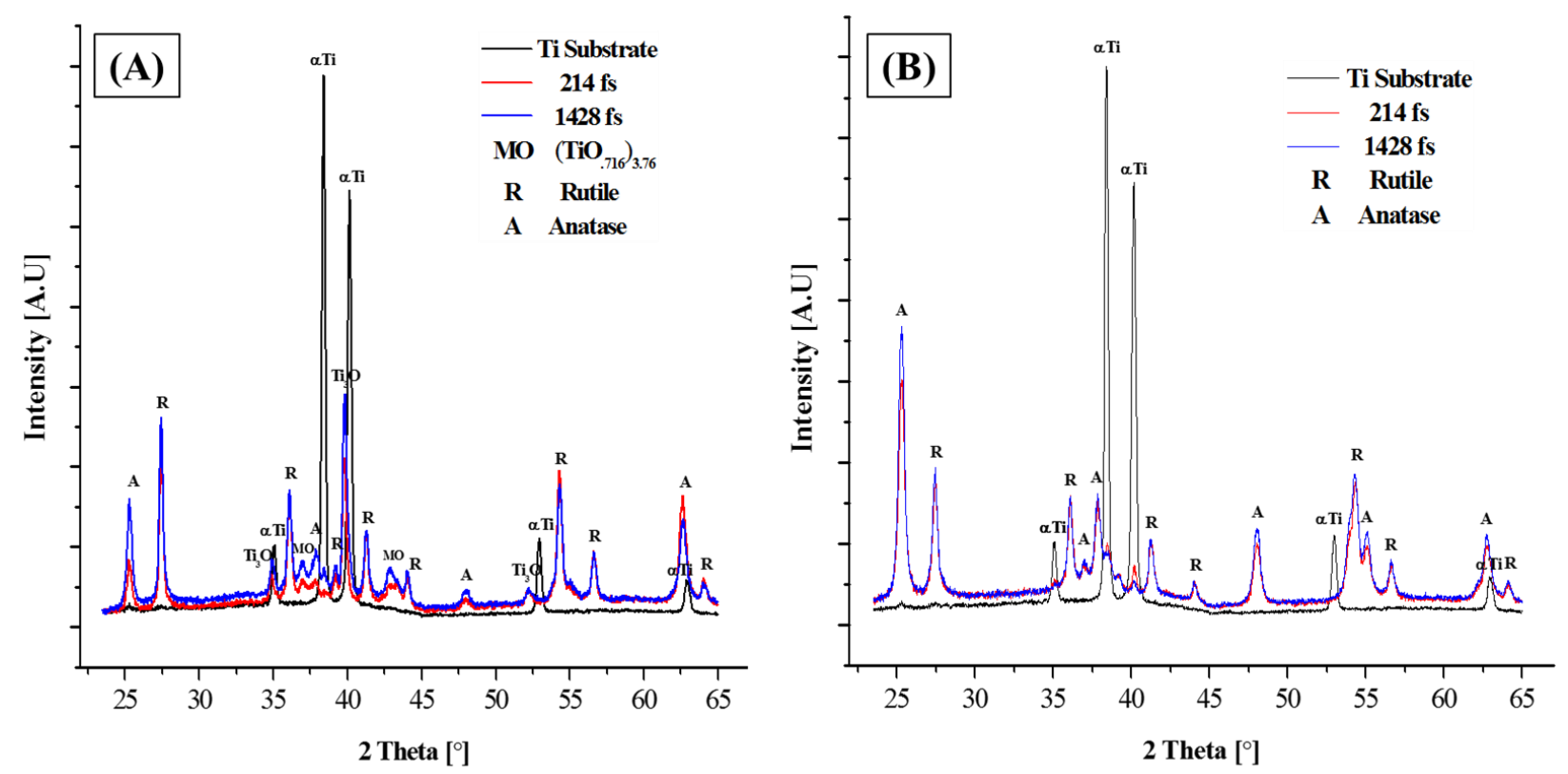

Figure 5.6: XRD patterns obtained for the multiphase nanomaterial synthesised at a laser fluence of (A) $5.14 \mathrm{~J} / \mathrm{cm}^{2}$, and (B) $1.64 \mathrm{~J} / \mathrm{cm}^{2}$. 
Furthermore, as observed and illustrated earlier in Figure 5.3, large sized nanospheres were synthesised at higher fluences. It is possible that during the course of the growth of such large nanostructures over a period of time, 'shielding' of the areas below the surface of the nanostructures from the Oxygen present in the ambient atmosphere could have led to lower Oxides of Titanium being formed. The XRD patterns in Figure 5.6(A) and Figure 5.6(B) also indicate that the pulse width did not have much influence on what type of phase constituted the nanomaterial. Apart from the above-mentioned phases, all the samples had XRD peaks corresponding to $\alpha$ Titanium present as well.

Rietveld fitting procedure of the XRD patterns was later done to quantitatively approximate the weight percentage of each of the different phases constituting the multiphase nano $\mathrm{TiO}_{\mathrm{x}}$ network, when synthesised at various laser conditions (Figure 5.7). The presence of greater amount of rutile was observed in the nano $\mathrm{TiO}_{\mathrm{x}}$ network formed at conditions of high temperature (i.e., high laser fluence) and high laser peak power (i.e., shorter pulse width) with a corresponding reduction seen in the amount of anatase phase. This is due to the fact that at higher temperatures the phase transformation of anatase to rutile occurs [22]. Another possible reason could be that, in larger particles $(>14 \mathrm{~nm}$ in size), which are more dominantly formed at conditions of high temperature, rutile is known grow at a faster rate and found to be more stable than anatase [96]. This is also in agreement with the observation made from the study of XPS spectrum. Furthermore, the presence of higher amount of anatase in smaller mean sized spherical nanostructures correlates with the fact that anatase has been reported to be more stable in small sized nanoparticles $(<50 \mathrm{~nm})$ and is known to transform to rutile once higher temperature or greater particle size is reached [22]. A slight increase (about 0.4 wt. \%) in the amount of the non-stoichiometric Oxide (TiO.716)3.76 was also seen at longer pulse widths in the Rietveld fitted values. 

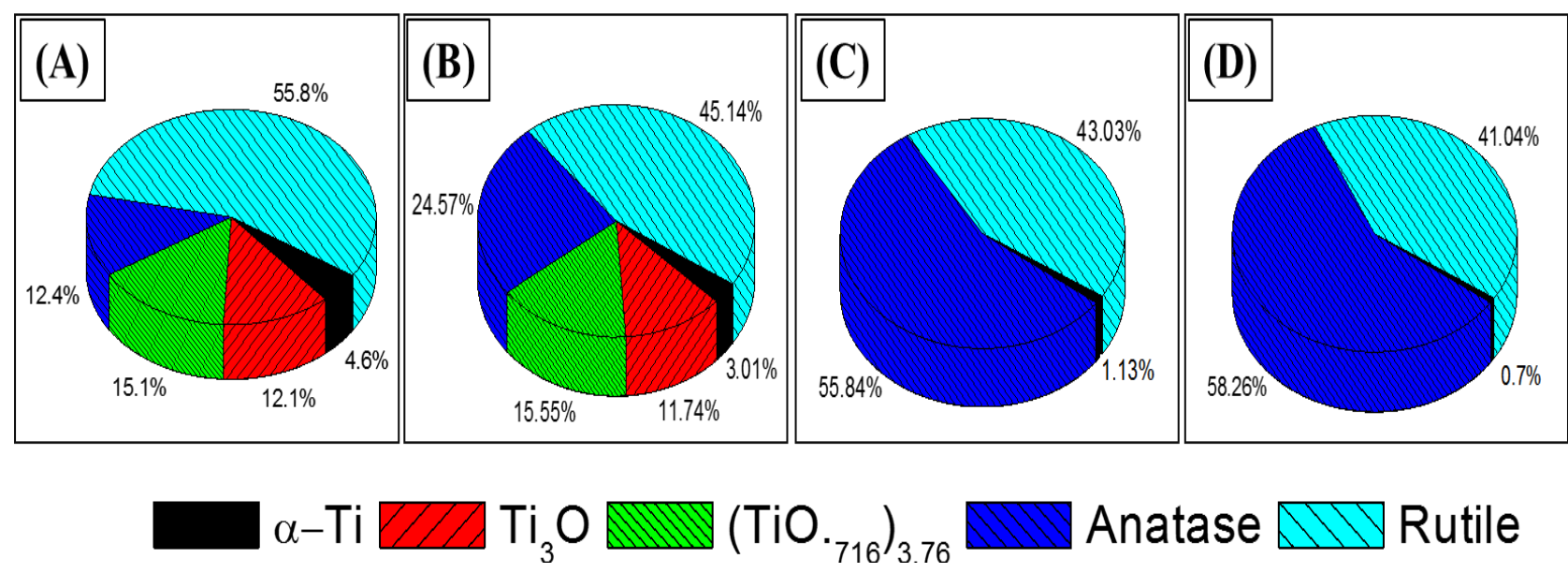

Figure 5.7: Pie charts showing the Rietveld fitted weight percentage values of all the identified phases for the multiphase nano $\mathrm{TiO}_{\mathrm{x}}$ network synthesised at a laser fluence and pulse width values of (A) $5.14 \mathrm{~J} / \mathrm{cm}^{2}, 214 \mathrm{fs}$, (B) $5.14 \mathrm{~J} / \mathrm{cm}^{2}, 1428 \mathrm{fs}$, (C) $1.64 \mathrm{~J} / \mathrm{cm}^{2}, 214 \mathrm{fs}$, and (D) $1.64 \mathrm{~J} / \mathrm{cm}^{2}, 1428 \mathrm{fs}$.

\subsection{Photon Absorption and Raman Enhancement}

The absorption properties of the photoabsorptive multiphase nano $\mathrm{TiO}_{\mathrm{x}}$ network was then measured. A steady increase in absorption in the visible and near infrared region of $300 \mathrm{~nm}$ to $1000 \mathrm{~nm}$ was observed. The absorption was much greater at wavelengths corresponding to the near infrared region between $800 \mathrm{~nm}$ to $1000 \mathrm{~nm}$, than at wavelengths corresponding to visible region of the solar spectrum. Figure 5.8 shows the absorption spectrum of the nano $\mathrm{TiO}_{\mathrm{x}}$ network synthesised at various laser conditions as well as that of base Titanium substrate. To better understand the impact that each of the multiple phases present in the nanomaterial might have had on its absorption properties, the weight percentage values of each of the phases present in the nanomaterial (as calculated by the Rietveld fitting procedure) was compared with the corresponding absorption spectrum that was obtained. 

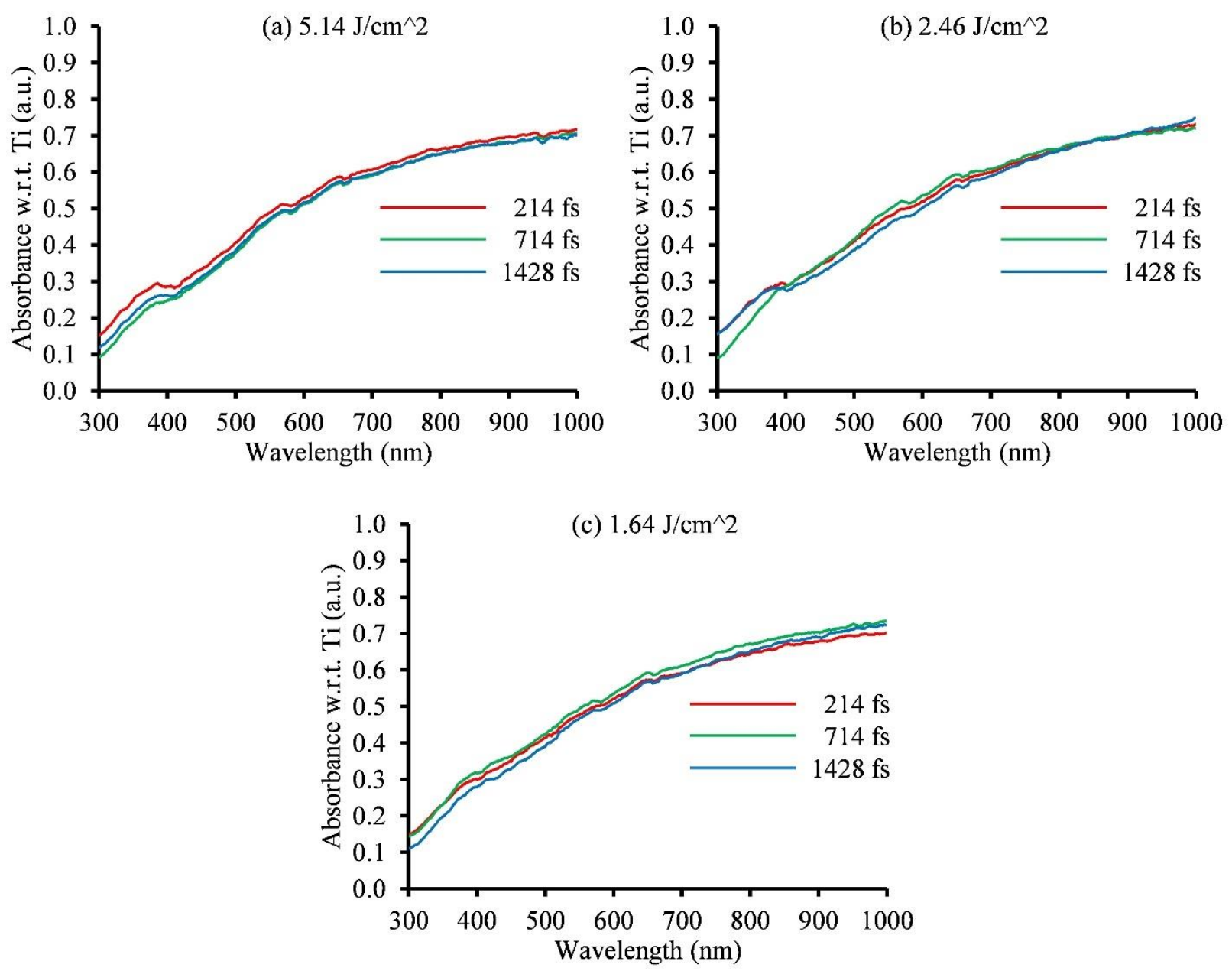

Figure 5.8: Absorption spectrum of the multiphase nano $\mathrm{TiO}_{\mathrm{x}}$ network synthesised at laser fluence of (a) $5.14 \mathrm{~J} / \mathrm{cm} 2$, (b) $2.46 \mathrm{~J} / \mathrm{cm} 2$ and (c) $1.64 \mathrm{~J} / \mathrm{cm} 2$ and different laser pulse widths.

Not much variation in the absorption spectrum was seen between nanomaterials synthesised at different lasing conditions. The significant enhancement and broadening of the absorption spectrum observed for the synthesised nanomaterial could be due to the resultant effect of the complex morphology of the three-dimensional nanomaterial network and the multiphase nature of the Titanium Oxides.

Raman Spectra were later obtained for the synthesised multiphase Titanium Oxide nanomaterial and the base Titanium substrate at two incident laser beam wavelengths: $532 \mathrm{~nm}$ and $785 \mathrm{~nm}$. 
Figure 5.9 shows the Raman spectra obtained for the base Titanium substrate and the multiphase nano $\mathrm{TiO}_{\mathrm{x}}$ synthesised at various laser conditions. While the Raman spectra of the base Titanium substrate contained no peaks, all the Raman spectra of the synthesised multiphase nanomaterial exhibited Raman peaks corresponding to anatase (at wavenumber $514 \mathrm{~cm}^{-1}$ ) and rutile (at wavenumbers $443 \mathrm{~cm}^{-1}$ and $610 \mathrm{~cm}^{-1}$ ). The other two lower Oxides of Titanium, found in the nanomaterial synthesised at higher laser fluences, $\mathrm{Ti}_{3} \mathrm{O}$ and $(\mathrm{TiO} .716)_{3.76}$, could not be identified via Raman spectroscopy due to their Raman inactive nature. This is because of the non-symmetric nature of their molecular structure. That results in no polarizability change in the molecule, and the subsequent absence of any Raman active vibration modes that are required to give a band in the Raman spectrum [97].

Another critical observation made in the acquired Raman spectra of the synthesised multiphase nano $\mathrm{TiO}_{\mathrm{x}}$ network was the significant amount of Raman enhancement attained at both laser beam wavelengths. This was done to further obtain evidence of the enhanced photon absorption observed earlier, as Raman scattering of photons is always preceded by the absorption of the incident photons by the molecules of the material [97]. As such, the significant Raman enhancement seen in the spectral peaks, with more enhancement at $785 \mathrm{~nm}$ than at $532 \mathrm{~nm}$, is also an indicator of the increment in photon absorption of the unique multiphase nano $\mathrm{TiO}_{\mathrm{x}}$ network at longer wavelengths. 

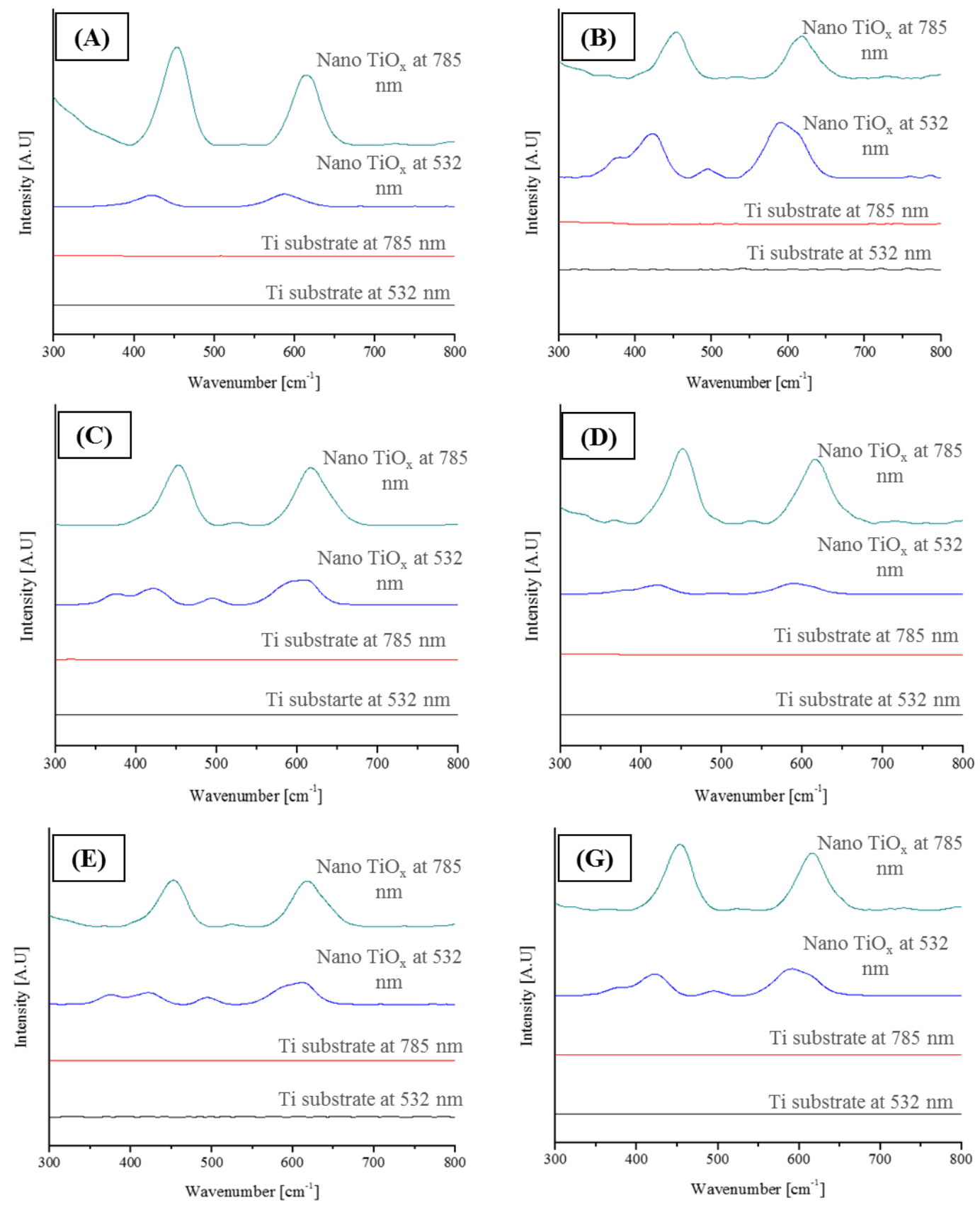

Figure 5.9: Raman Spectra obtained at two laser beam wavelengths $(532 \mathrm{~nm}$ and $785 \mathrm{~nm}$ ) for both the base Titanium substrate and the multiphase nano $\mathrm{TiO}_{\mathrm{x}}$ at laser fluence and pulse width values of (A) $5.14 \mathrm{~J} / \mathrm{cm}^{2}, 214 \mathrm{fs}$,

(B) $5.14 \mathrm{~J} / \mathrm{cm}^{2}, 1428 \mathrm{fs}$, (C) $2.46 \mathrm{~J} / \mathrm{cm}^{2}, 214 \mathrm{fs}$, (D) $2.46 \mathrm{~J} / \mathrm{cm}^{2}, 1428 \mathrm{fs}$, (E) $1.64 \mathrm{~J} / \mathrm{cm}^{2}, 214 \mathrm{fs}$

(F) $1.64 \mathrm{~J} / \mathrm{cm}^{2}, 1428 \mathrm{fs}$. The Raman spectra of the base Titanium substrate obtained at $532 \mathrm{~nm}$ and $785 \mathrm{~nm}$ laser beam wavelength is indicated in black and red colour respectively. The Raman spectra of the nanomaterial obtained at $532 \mathrm{~nm}$ and $785 \mathrm{~nm}$ laser beam wavelength is indicated in blue and olive green colour respectively. 


\subsection{Summary}

In this chapter, we report a Titanium Oxide photoabsorptive nanomaterial that is uniquely multiphase and has demonstrated the ability to effectively absorb photons in the deeper visible as well as the Near Infrared region of the solar spectrum. The absorption spectrum obtained for this multiphase nanomaterial revealed a three-fold enhancement in the deeper visible region of the spectrum and a six-fold enhancement in the NIR region when compared to the base Titanium substrate. This dopant free and three dimensionally arranged nanomaterial was determined to be distinctively hybrid in nature and comprised of multiple phases of Titanium: $\mathrm{Ti}_{3} \mathrm{O},\left(\mathrm{TiO}_{.716}\right)_{3.76}$, rutile and anatase. This photoabsorptive multiphase nano $\mathrm{TiO}_{\mathrm{x}}$ network was synthesised by a unique fusion of plume formation and vapour condensation mechanisms, brought about by the interaction of ultra-short laser pulses and base Titanium substrate. The weight percentage of each of these phases of Titanium Oxide was shown to vary with the nature of interaction, which governed the thermal and physical conditions at the time of formation. This study thus presents a photoabsorptive and dopant free multiphase Titanium Oxide nanomaterial with a broadened absorption spectrum extending to deeper visible and Near Infrared region of the solar spectrum. The potential applications of this multiphase nanomaterial with such an enhanced and broadened photon absorption capability, can include its implementation and integration in photon harvesting and other nano engineered systems. 


\section{Chapter 6}

\section{Plasmon Hybridization of Multiphase}

\section{Nano TiOx Networks}

Photoabsorptive nanomaterials in the form of single layered plasmonic nanostructures and quantum dots (QDs) are being employed to boost the absorption of light by exploiting the plasmon resonance [98]-[101] and quantum confinement effect respectively [102]-[104]. However, their applications have been limited due to very narrow absorption bandwidth that is strongly dependent and limited to their Plasmon resonant peaks, as in case of plasmonic nanostructures [105], or the band gap and amount of stacking, as in case of quantum dots [106], [107]. As such there is a paucity of nanomaterials which can exhibit a broadened and enhanced absorption bandwidth that can be easily fabricated and do not require additional cost bearing steps like doping or multiple stacking.

In this chapter, a plasmon hybridised nano $\mathrm{TiO}_{\mathrm{x}}$ that has demonstrated an enhanced photon absorption capability over a wide range of the solar spectrum, extending from visible to near infrared wavelengths $(300-1000 \mathrm{~nm})$ is outlined. To serve as a template, the self-assembled multiphase nano $\mathrm{TiO}_{\mathrm{x}}$ network described in Chapter 5 was utilised. This nanoporous, cross linked self-assembled network of nanomaterial fabricated on a base Titanium substrate and having multiple resonant optical modes due to its complex structure, had effectively displayed the ability to harvest photons over deeper visible and near infrared region of the solar spectrum. 
In plasmonic metal nanomaterials, conduction electrons oscillate collectively at resonant frequency, which cause the incident light to be strongly scattered or absorbed [98]. This characteristic property is thus utilized in trapping of photons of different wavelengths, by correspondingly tuning the resonant frequency of the nanoparticles, which is a known function of the particle size, shape, material and the refractive index of surrounding medium [98], [108]-[110]. Resonances in between the resonant frequencies of two individual metals can also be achieved by alloying of metal particles, which further provide an opportunity to broaden the absorption spectrum [105].

Gold $(\mathrm{Au})$ monolayer nanoparticles which only exhibit a plasmon resonance peak in the visible region were exploited and amalgamated to induce the localised surface plasmon resonance (LSPR) behavior and sensitize the self-assembled nanomaterial to expand its absorption spectrum in the visible region. This utilisation of the LSPR effect in multiphase nanomaterial was done via the fabrication of a plasmon hybridised multiphase nano $\mathrm{TiO}_{\mathrm{x}}$ that is multilayered and three dimensional. Since Resonances in between the resonant frequencies of two individual metals can also be achieved by alloying of metal particles, which further provide an opportunity to broaden the absorption spectrum [105], Gold/Palladium (Au/Pd) alloy plasmon hybridised nanomaterial was also fabricated and studied. Assessment of the influence of $\mathrm{Au}$ and $\mathrm{Au} / \mathrm{Pd}$ plasmon hybridisation on the nanomaterial was conducted by varying the degree of hybridisation to optimize the surface plasmon coupling at the interface of the metal nanoparticles and core nanomaterial. To incorporate further controllability and study the feasibility of tuning the optical properties of the hybridised nanomaterial towards broadband photon absorption, the effect of change in the phase composition and average size of the individual nanospheres constituting the nanomaterial network on the absorption spectrum was also studied. 


\subsection{Induction of Localized Surface Plasmon Behaviour Using Au and Au/Pd Alloy}

The core multiphase nano $\mathrm{TiO}_{\mathrm{x}}$ network was initially synthesised by an ultrashort laser pulses, as described in Chapter 5 and seen in Figure 6.1 (A). The laser beam was scanned at a speed of $5 \mathrm{~mm} / \mathrm{s}$ on the Titanium samples at effective laser fluence values of $5.14 \mathrm{~J}, 2.46 \mathrm{~J}$ and $1.64 \mathrm{~J}$ per unit square centimeter. The corresponding repetition rates were $4 \mathrm{MHz}, 8.36 \mathrm{Mhz}$ and $12.54 \mathrm{MHz}$ respectively. To incorporate an additional study variable, the duration of ultrashort laser pulses was varied, and the experiments were conducted at laser pulse width values of $214 \mathrm{fs}, 714 \mathrm{fs}$ and 1428 fs. The multiphase nano $\mathrm{TiO}_{\mathrm{x}}$ was then hybridised with $\mathrm{Au}$ and $\mathrm{Au} / \mathrm{Pd}$ alloy nanoparticles and nano islands, as seen in Figure 6.1(B) and(C) respectively, to induce the phenomenon of localized surface plasmon resonance (LSPR). The hybridisation was done via plasma assisted deposition process, by using a Sputter Coater (Quorum SC 7620) for various time durations using $\mathrm{Au}$ and $\mathrm{Au} /$ palladium alloy targets. Two sample sets of plasmon hybridised multiphase nano $\mathrm{TiO}_{\mathrm{x}}$ nanomaterial decorated with noble metal nanoparticles of $\mathrm{Au}$ and Au/Pd alloy (Au:Pd::80:20) of varying atomic $\%$ concentrations were thus generated. The atomic concentrations were varied by choosing two plasma assisted deposition times: $30 \mathrm{~s}$ and $60 \mathrm{~s}$. The atomic concentrations were then measured using EDX point scan. The first set of core self-assembled nanomaterial samples, synthesised at all the previously stated laser parameter conditions, were coated with average $\mathrm{Au}$ atomic concentrations of $1.74 \%$ and $3.56 \%$ (corresponding to deposition times of $30 \mathrm{~s}$ and $60 \mathrm{~s}$ respectively). The second set of core self-assembled nanomaterial samples, again synthesised at all the previously stated laser parameter conditions, were coated with $\mathrm{Au} / \mathrm{Pd}$ alloy having average $\mathrm{Au}$ atomic concentrations of $0.82 \%, 1.63 \%$ and the corresponding palladium atomic 
concentrations of $0.30 \%, 0.68 \%$ respectively (corresponding to deposition times of $30 \mathrm{~s}$ and $60 \mathrm{~s}$ respectively).

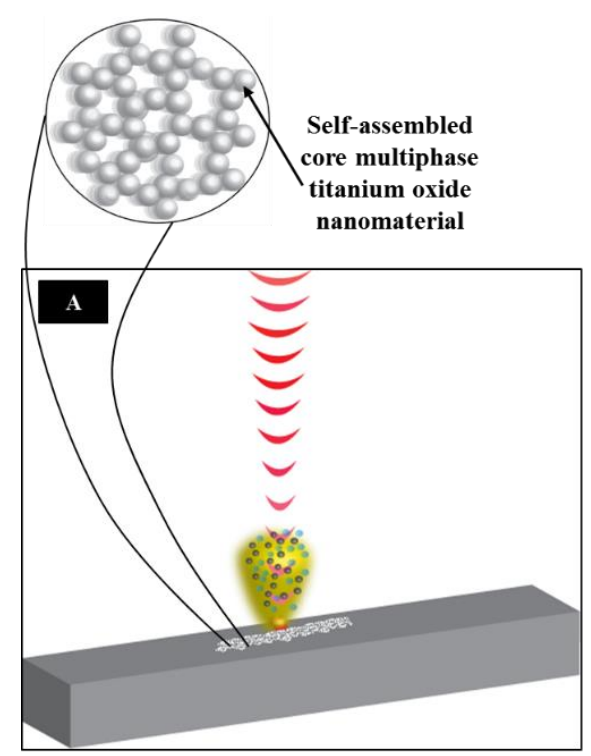

Ultrafast laser pulse induced selfassembly of core nanomaterial

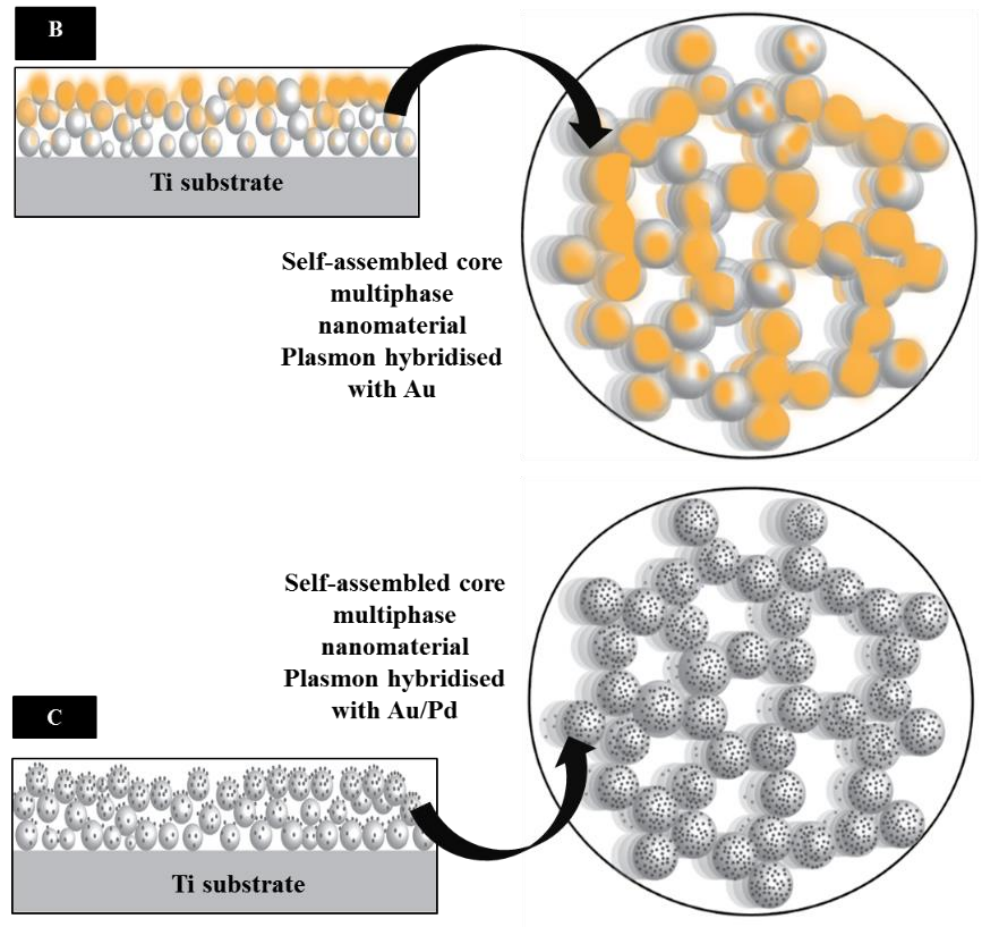

Figure 6.1:(A) Graphical illustration of the fabrication mechanism of the core self-assembled multiphase nano $\mathrm{TiO}_{\mathrm{x}}$ followed by its plasmon hybridisation with (B) Au and (C) Au/Pd alloy.

\subsection{Surface Morphological Characterization}

The study of the morphology of the Au and Au/Pd alloy plasmon hybridised nanomaterial revealed that the deposition of the above noble metals was non-uniform along the depth of the 3D nanomaterial network. The Au atoms deposited on the nanomaterial formed a thin layer of coalesced large islands, covering a large area of topmost layer of the 3D network. This can be 
attributed to the tendency of Au atom to agglomerate, due to their high surface tension [111]. At deeper levels of the nanomaterial network, widely space, smaller islands of Au were observed. Samples hybridised with $\mathrm{Au} / \mathrm{Pd}$ alloy on the contrary, indicated a uniform deposition on the nanomaterial, consisting of compact, mostly spherical, particles devoid of any significant coalesced large island formation. This is because palladium acts as a physical barrier and prevents the inherent tendency of atoms of Au to coalesce and form clusters. As with Au hybridised nanomaterial, the extent of deposition was lesser at greater depths of the nanomaterial network.

Figure 6.2(A) shows the back scattered SEM image of the Au plasmon hybridised multiphase nano $\mathrm{TiO}_{\mathrm{x}}$ nanomaterial synthesised at laser fluence of $5.14 \mathrm{~J} / \mathrm{cm}^{2}$ and pulse duration of $214 \mathrm{fs}$. Figure 6.2(A1) and Figure 6.2(A2) show the corresponding secondary electron images taken at a higher magnification. The brighter areas covering the nanostructured network indicate the higher average atomic numbered Au that is seen forming large coalesced islands at the top but reducing to nanoscale spherical islands at the bottom of the network. Figure 6.2(B) shows the Au/Pd alloy plasmon hybridised multiphase Titanium Oxide nanomaterial synthesised at a laser fluence of $5.14 \mathrm{~J} / \mathrm{cm}^{2}$ and pulse duration of $1428 \mathrm{fs}$ having a uniform deposition with no large coalesced island formations. Figure 6.2(B1) and Figure 6.2(B2) show the corresponding secondary electron images taken at a higher magnification. 

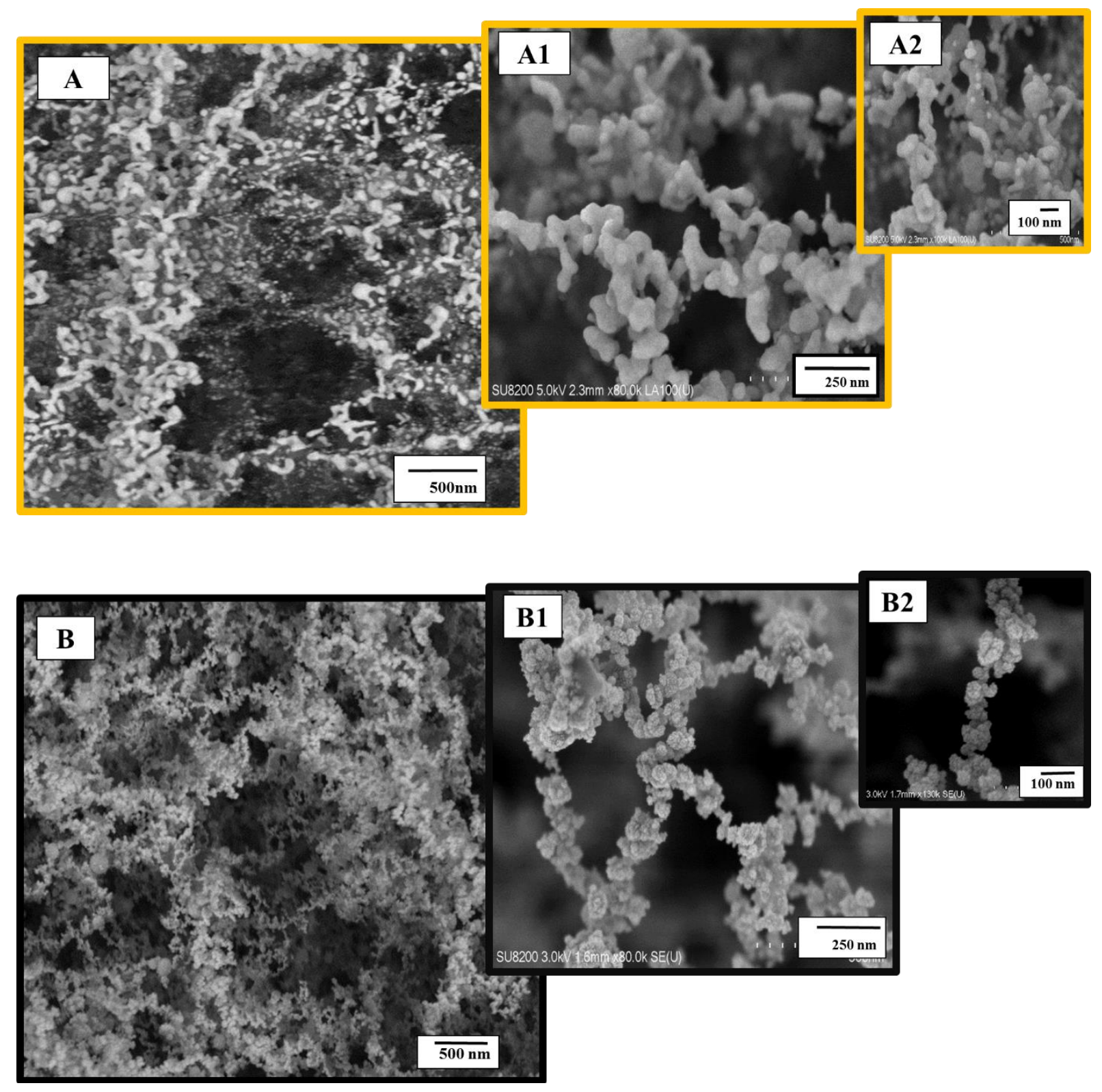

Figure 6.2: SEM images of the self-assembled 3D network of core Titanium Oxide multiphase nanomaterial plasmon hybridised with (A) Au, and (B) Au/palladium alloy.

The following formula was used to estimate the maximum possible thickness of both Au and $\mathrm{Au} / \mathrm{Pd}$ alloy plasma deposited on the top surface of the multiphase nano $\mathrm{TiO}_{\mathrm{x}}[112]$ :

$$
D=K_{\mathrm{m}} I_{\mathrm{s}} t
$$


where $\mathrm{D}$ is the thickness of the deposition, $K_{\mathrm{m}}$ is the material constant in argon gas (for $\mathrm{Au}$, $\mathrm{K} \sim 0.17$ and for $\mathrm{Au} / \mathrm{Pd} \mathrm{K} \sim 0.16$ ), $I_{\mathrm{s}}=18 \mathrm{~mA}$ is the sputtering current (adjusted by argon gas partial pressure in the sputter coater) and $t$ is the deposition time chosen to get different atomic percentages concentrations of $\mathrm{Au}$ and $\mathrm{Au} / \mathrm{Pd}$.

Taking the approximate deposition rate for Au atoms into consideration, it was deduced that the maximum thickness of the Au layer on the top surface of the multiphase nano $\mathrm{TiO}_{\mathrm{x}}$ network could not be more than $9 \mathrm{~nm}$ for the nanomaterial samples deposited with an average Au atomic concentration of $1.74 \%$ and $18 \mathrm{~nm}$ for the samples with an average Au atomic concentrations of $3.56 \%$. Likewise, due to a lower deposition rate for the heavier $\mathrm{Au} / \mathrm{Pd}$ alloy, the maximum thickness would not exceed $8.5 \mathrm{~nm}$ and $17 \mathrm{~nm}$ for the samples deposited with an atomic concentration of $\mathrm{Au} 0.82 \%, \mathrm{Pd} 0.30 \%$ and $\mathrm{Au} 1.63 \%, \mathrm{Pd} 0.68 \%$ respectively.

As LSPR is a function of the size of the noble metal plasmon nanoparticles [113], it is imperative to distinguish and observe the extent of coverage of the individual Titanium Oxide nanospheres of the core nanomaterial by the plasmonic $\mathrm{Au}$ and $\mathrm{Au} / \mathrm{Pd}$ nanoparticles. TEM images of the nanomaterial samples were thus taken to better predict their surface plasmon behaviour. Figure 6.3(A) shows the core non-hybridised nanomaterial synthesised at a laser fluence of $1.54 \mathrm{~J} / \mathrm{cm}^{2}$ and pulse duration of $1428 \mathrm{fs}$, which is subsequently plasmon hybridised with Au (Figure 6.3(B)) and Au palladium alloy (Figure 6.3(C)). The highly-contrasted images clearly indicate the darker $\mathrm{Au}$ and $\mathrm{Au} /$ palladium islands on the lighter multiphase nano $\mathrm{TiO}_{\mathrm{x}}$ nanospheres. The greater tendency of $\mathrm{Au}$ atoms to agglomerate and form coalesced islands than $\mathrm{Au} / \mathrm{Pd}$ is also seen in Figure 6.3(B) and (C). 


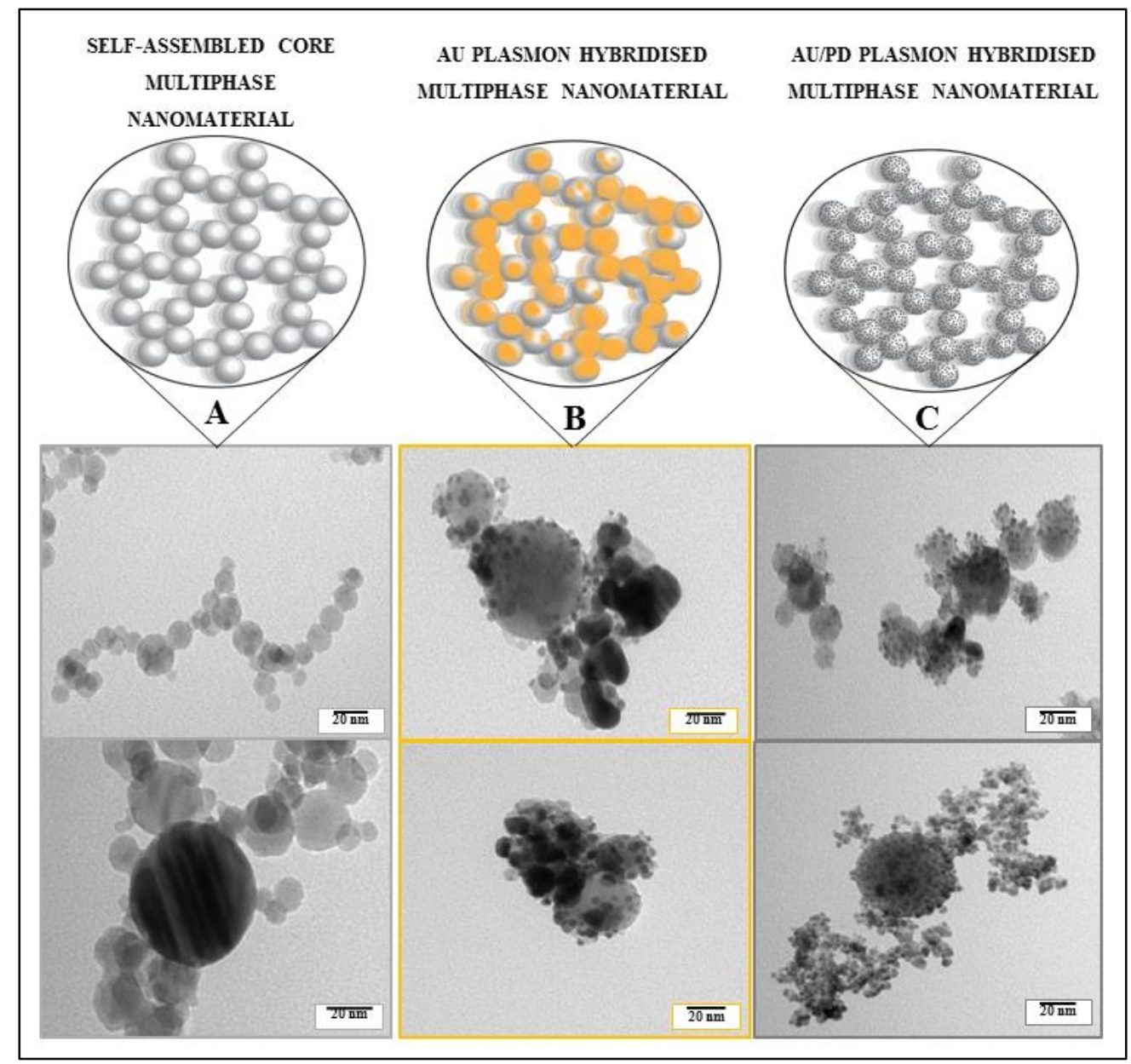

Figure 6.3: TEM images of the (A) Self-assembled core nano $\mathrm{TiO}_{\mathrm{x}}$ nanomaterial consisting of aggregated nanospheres which are subsequently plasmon hybridised with (B) Au and (C) Au/Pd alloy.

Raman spectra for the plasmon hybridised nanomaterial synthesised at various laser processing conditions was later obtained at two laser beam wavelengths: 532 and $785 \mathrm{~nm}$. Figure 6.4 shows the Raman spectra at laser beam wavelength $532 \mathrm{~nm}$, for the nanomaterial synthesised at a laser fluence of $1.64 \mathrm{~J} / \mathrm{cm}^{2}$ and pulse width value of $1428 \mathrm{fs}$ and plasmon hybridised with different atomic concentrations of $\mathrm{Au}$ and $\mathrm{Au} / \mathrm{Pd}$. The spectra of base Titanium substrate plasma deposited with a layer of noble metal nanoparticles was also acquired and found to have no Raman peaks due to their Raman inactive nature. The non-hybridised core multiphase Titanium Oxide 
nanomaterial exhibited high intensity Raman peaks corresponding to both anatase $\left(513 \mathrm{~cm}^{-1}\right)$ and rutile $\left(447 \mathrm{~cm}^{-1}\right.$ and $\left.612 \mathrm{~cm}^{-1}\right)$. No Raman peaks were observed for the other Oxides constituting the nanomaterial, as both $\mathrm{Ti}_{3} \mathrm{O}$ and (TiO.716) 3.76 are Raman inactive due to non-symmetric nature of their molecular structure [97]. For the nano $\mathrm{TiO}_{\mathrm{x}}$ plasmon hybridised with $\mathrm{Au}$ and $\mathrm{Au} / \mathrm{Pd}$ alloy, the intensity of those peaks reduced progressively as the amount of plasma metal deposition was increased, until no peaks were seen due to the shielding effect of the increased metal coating. The Raman peaks for the nanomaterial hybridised with $\mathrm{Au} / \mathrm{Pd}$ were more pronounced since shielding effect of the Raman signal was less inhibited. This is due to the fill factor being reduced by the smaller size of the Au/Pd nanoparticles. This can be clearly observed in Figure 6.4(A) and (B).

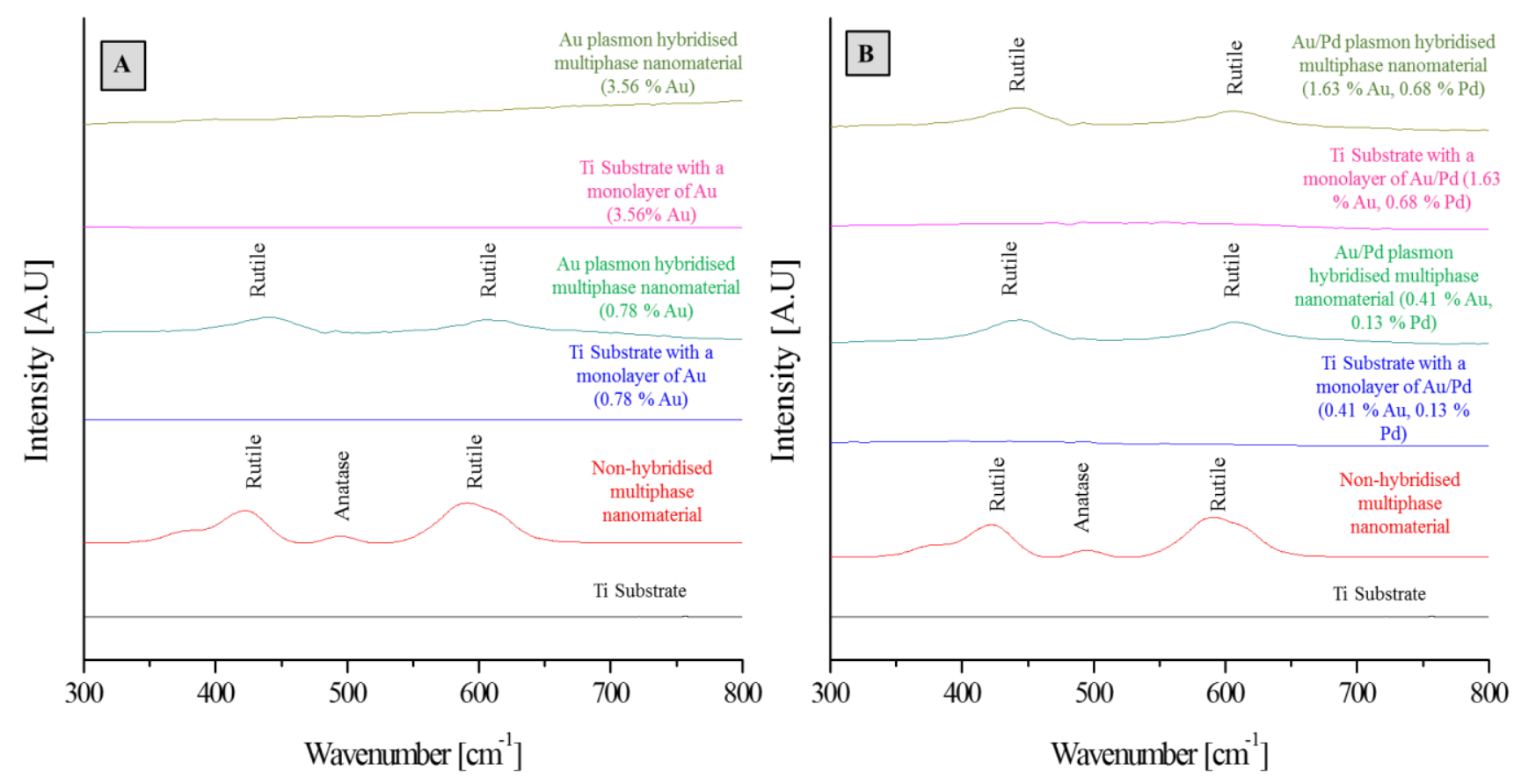

Figure 6.4: Raman spectra obtained at a laser beam wavelength of $532 \mathrm{~nm}$ for the multiphase nano $\mathrm{TiO}_{\mathrm{x}}$ before and after its plasmon hybridisation with (A) $\mathrm{Au}$, and (B) Au/Pd alloy. The Raman spectra for the base Titanium substrate with and without the noble metal hybridisation has also been plotted. 


\subsection{Enhanced Photon Absorptive Behaviour}

The absorption spectrum of the synthesised core self-assembled nano $\mathrm{TiO}_{\mathrm{x}}$ before and after plasmon hybridisation was acquired and plotted. The resultant spectrum was also compared to the absorption of three control surfaces. The first being the base Titanium substrate with no noble metal deposition or nanomaterial present on it, the second and third being only the base Ti substrate, covered with a monolayer of $\mathrm{Au}$ and $\mathrm{Au} / \mathrm{Pd}$ alloy of the same atomic \% concentration as that deposited on the nanomaterial being studied.

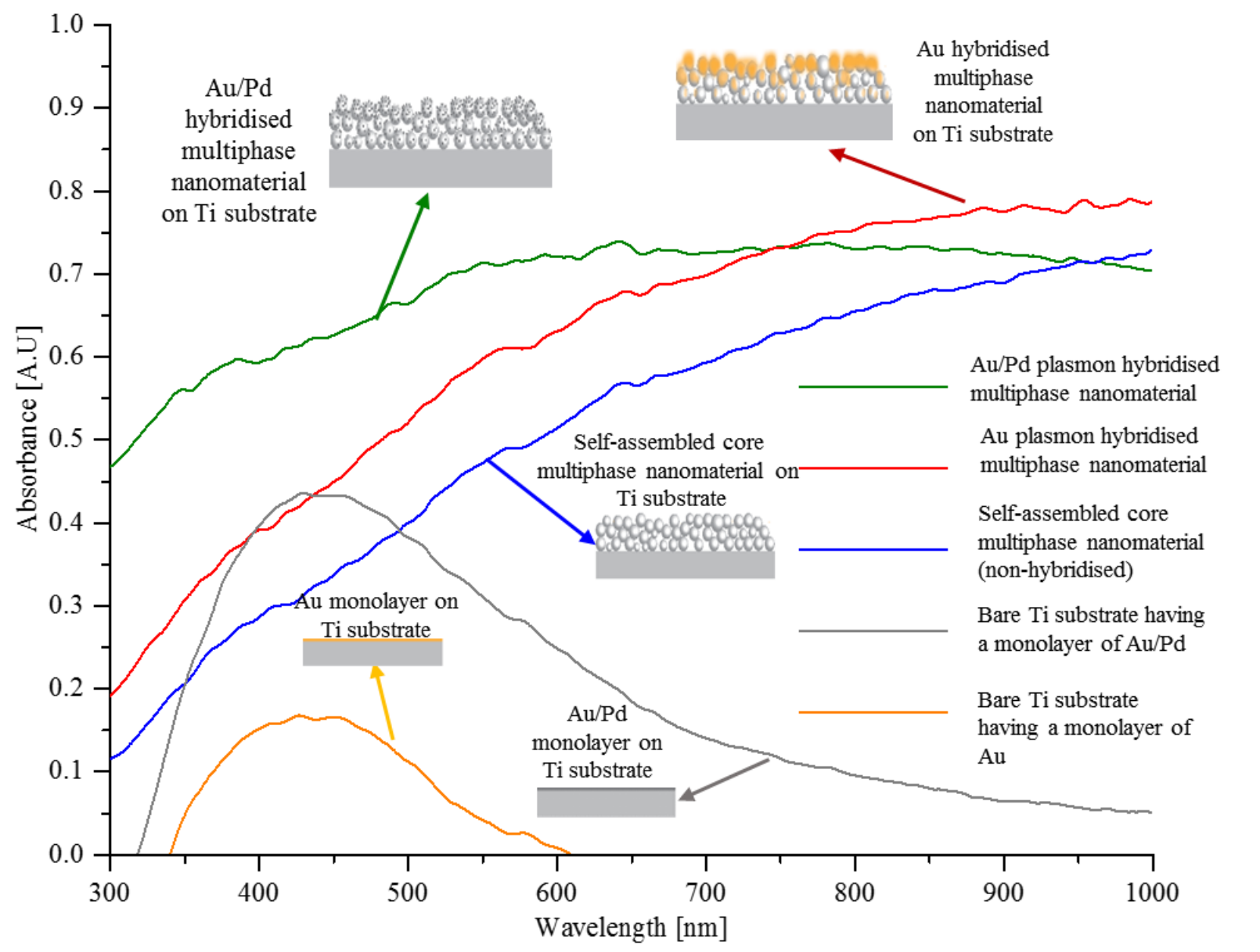

Figure 6.5: Broadening and enhancement observed in the absorption spectrum of the plasmon hybridised multiphase nano $\mathrm{TiO}_{\mathrm{x}}$ with respect to titanium substrate. 
Figure 6.5 shows the absorption spectrum of all the above-mentioned samples. The core multiphase nano $\mathrm{TiO}_{\mathrm{x}}$ was synthesised at a laser fluence of $1.64 \mathrm{~J} / \mathrm{cm}^{2}$ and pulse width of $1428 \mathrm{fs}$. The base Titanium substrate covered with a monolayer of Au showed the plasmon resonance peak centred at $430 \mathrm{~nm}$, while the substrate covered with a monolayer of $\mathrm{Au} / \mathrm{Pd}$ alloy showed a much more broadened peak centred on the same wavelength. The obvious shift from the characteristic SPR band of Au nanoparticles, widely reported to be around $520 \mathrm{~nm}$, could be due to the influence of the dielectric properties of the surrounding medium, namely air and Titanium substrate [114]. As clearly indicated, the nano $\mathrm{TiO}_{\mathrm{x}}$ exhibited an absorption spectrum more enhanced than the base Titanium substrate, with almost a six-fold increment seen at wavelengths greater than $650 \mathrm{~nm}$, which was consistent with the previous study described in Chapter 5. This enhancement was attributed to the characteristic multiphase nature of the synthesised core Titanium Oxide nanomaterial, comprising of the following Oxide phases of Titanium: rutile, anatase, $\mathrm{Ti}_{3} \mathrm{O}$ and non-stoichiometric (TiO.716)3.76 [115].

Another influential factor for the enhanced photon absorptive behaviour of the self-assembled nanomaterial could be the three dimensionality of the network structure and the 'web-like' morphology that renders the multiphase Titanium Oxide nanomaterial to behave like a penetrable porous material. This complex three dimensional structure of the aggregated nanospheres to form the core semiconductor nanomaterial network, could have contributed towards the improvement of the optical absorptive properties of the nanomaterial. This could be a result of delocalization and collective electronic states formation that could have arisen due to the close interaction between the individual aggregated nanospheres in the 3D network [116].

The range of absorption of the Au plasmon hybridised nanomaterial was as broad as that of the non-hybridised core multiphase nanomaterial, ranging from 300-1000 nm, but the measured 
amount of absorbance was almost double of that of the non-hybridised nano $\mathrm{TiO}_{\mathrm{x}}$. Furthermore, the measured absorption spectrum of the $\mathrm{Au} / \mathrm{Pd}$ alloy plasmon hybridised nanomaterial was seen to have a distinct absorption spectrum, with more absorption than the Au plasmon hybridised nanomaterial in the visible range $(300-750 \mathrm{~nm})$, but a slightly reduced absorbance observed for the Near Infrared Region (NIR) (800-1000 nm). It is known that the surface plasmon resonance in the metal nanoparticle islands leads to charge separation in the semiconductor material, followed by the injection of electrons into its conduction band from the metal nanoparticle, causing the formation of a Schottky barrier and leading to greater electron transfer and reduced recombination [117]. As such, the enhanced absorption spectrum observed for the multiphase Titanium Oxide nanomaterial plasmon hybridised with $\mathrm{Au}$ and $\mathrm{Au} / \mathrm{Pd}$ alloy, could be a consequence of this plasmonic surface interaction between the coated nano sized noble metal plasmon islands and the core Titanium Oxide nanomaterial. The considerably higher absorption seen for $\mathrm{Au} / \mathrm{Pd}$ hybridised nanomaterial in the visible range $(300-750 \mathrm{~nm})$, in comparison to the $\mathrm{Au}$ plasmon hybridised nanomaterial, could be due to the combined surface plasmon effect contributions of both palladium and $\mathrm{Au}$ and also due to the smaller size of the $\mathrm{Au} / \mathrm{Pd}$ islands on the nanomaterial than the large coalesced $\mathrm{Au}$ islands formed due to Au hybridisation. This is in agreement with previous widely reported studies that increase in the size of noble metal nanoparticles leads to a red shift of their plasmonic absorption, as seen in our multiphase nano $\mathrm{TiO}_{\mathrm{x}}$ plasma coated with $\mathrm{Au}$, due to shifting of peak modes to lower energies [118].

Furthermore, the change in morphology of the multiphase nanomaterial network due to the plasmon hybridisation of its individually aggregated nanospheres, with $\mathrm{Au}$ and $\mathrm{Au} / \mathrm{Pd}$ metal nanoparticles and nano islands, results in enhanced photon absorptive behaviour. The size and 
shape of such plasmon metal nanoparticles on the core nanomaterial determine their absorption and scattering cross section, as established by the Mie theory in the following equations [119]:

$$
\begin{gathered}
C_{a b s}=\frac{2 \pi}{\lambda} \operatorname{Im}\left[\alpha_{d}\right] \\
C_{\text {sca }}=\frac{1}{6 \pi}\left(\frac{2 \pi}{\lambda}\right)^{4}\left|\alpha_{d}\right|^{2}
\end{gathered}
$$

where, $C_{\text {abs }}$ and $C_{\text {sca }}$ are the absorption and scattering cross section of the metal nanoparticles and Im indicates the imaginary part of the polarizability of the metal nanoparticle, $\alpha_{d}$, given by [98]:

$$
\alpha_{d}=\left(1+\kappa_{s}\right) \varepsilon_{0} V \frac{\left[\varepsilon(\omega)-\varepsilon_{\mathrm{m}}\right]}{\left[\varepsilon(\omega)+\kappa_{s} \varepsilon_{\mathrm{m}}\right]}
$$

where, $\varepsilon(\omega)$ is the complex dielectric function of the particle, $V$ is the volume of the particle, $\varepsilon_{\mathrm{m}}$ is the medium dielectric constant, $\varepsilon_{0}$ is the permittivity of free space and $\kappa_{s}$ is the shape factor. The above equations exhibit that absorption process dominates smaller metal particles while scattering is more pronounced in larger metal particles [119]. As such, a combination of scattering and absorption phenomena over the entire range of the three-dimensional nanomaterial network, due to difference in the extent of coverage of the nanomaterial by the plasmon noble metal nanoparticles, could have resulted in more photons being trapped and harvested. This extent of coverage and hence hybridisation of the nanomaterial by the plasmon noble metal nanoparticles and nano islands is a function of both the noble metal atomic concentration, as well as the position of the aggregated nanospheres within the nanomaterial network. The top of the network would obviously be covered to a larger extent than the subsequent layers at the bottom. Furthermore, in Figure 6.6 and Figure 6.7, it is clearly seen that the extent of coverage of the nanospheres of multiphase Titanium Oxide nanomaterial by the $\mathrm{Au}$ and $\mathrm{Au} / \mathrm{Pd}$ nanoparticles is greater for higher 
atomic $\%$ concentration hybridisation of the noble metal nanoparticles on the nanomaterial. This results in scattering being the most dominant extinction phenomena where large islands of the plasma coated metal on the multiphase nanomaterial are present and the absorption process dominating at places where smaller size of the metal islands cover the multiphase nanomaterial [105]. Additionally, the greater absorption seen in the $\mathrm{Au} / \mathrm{Pd}$ alloy hybridised nanomaterial than the Au hybridised nanomaterial could be due to the more spherical/hemi spherically shaped $\mathrm{Au} / \mathrm{Pd}$ nanoparticles, as observed in all the TEM images. This is because such symmetrically shaped metal nanoparticles have a single dipolar plasmon resonance, with all their dipolar modes being degenerate, thereby leading to enhanced scattering and trapping of photons [120], [121]. If the fill factor $f_{\mathrm{f}}$ is taken to be as,

$$
f_{\mathrm{f}}=\frac{V_{\text {islands }}}{V_{\text {core }}}
$$

where $V_{\text {core }}$ is the average volume fraction of the core multiphase Titanium Oxide nanosphere and $V_{\text {islands }}$ is the average volume fraction of the $\mathrm{Au}$ or $\mathrm{Au} / \mathrm{Pd}$ plasmon metal islands on the core nanomaterial, it is observed that as atomic percentage concentration and coverage of the Au or $\mathrm{Au} / \mathrm{Pd}$ plasmon islands on the core nano $\mathrm{TiO}_{\mathrm{x}}$ increases, the fill factor value also increases. 


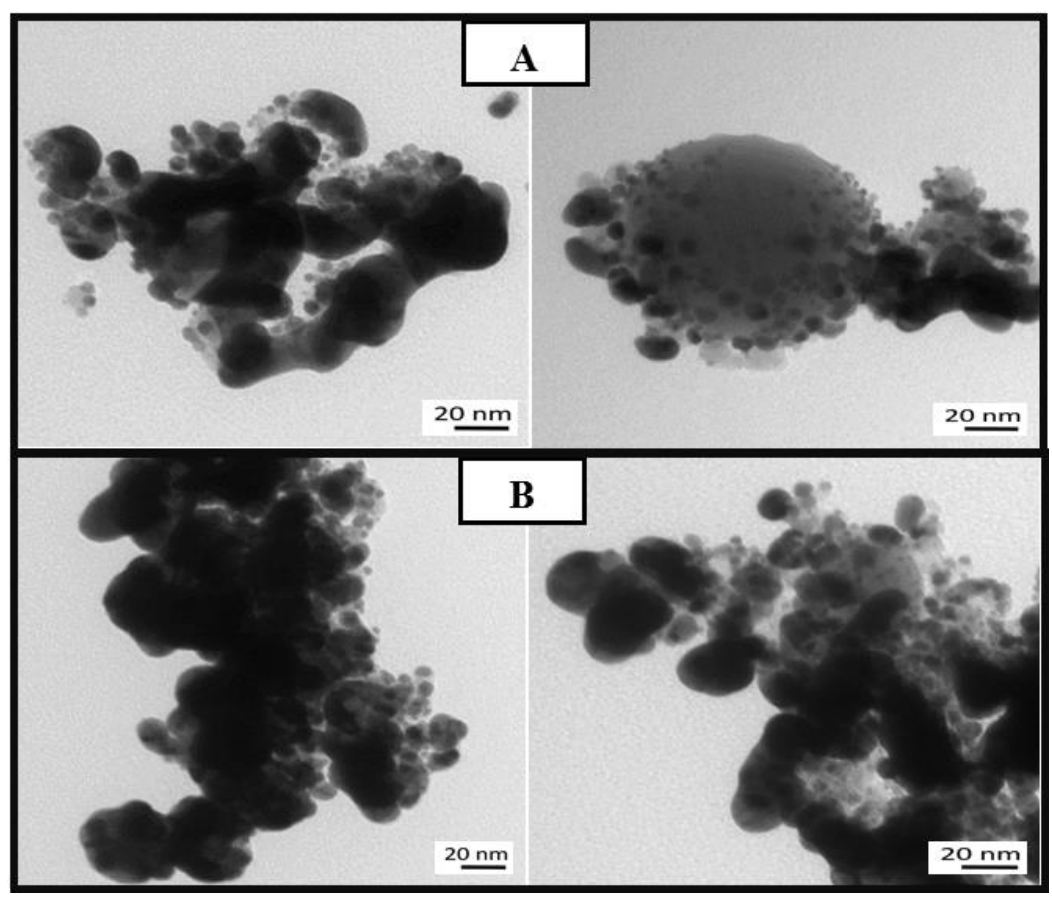

Figure 6.6: Variation in the degree of coverage and hybridisation of the core multiphase nano $\mathrm{TiO}_{\mathrm{x}}$ by coalesced islands of $\mathrm{Au}$, at different atomic \% concentrations of (A) $1.74 \%$, and (B) $3.56 \%$.

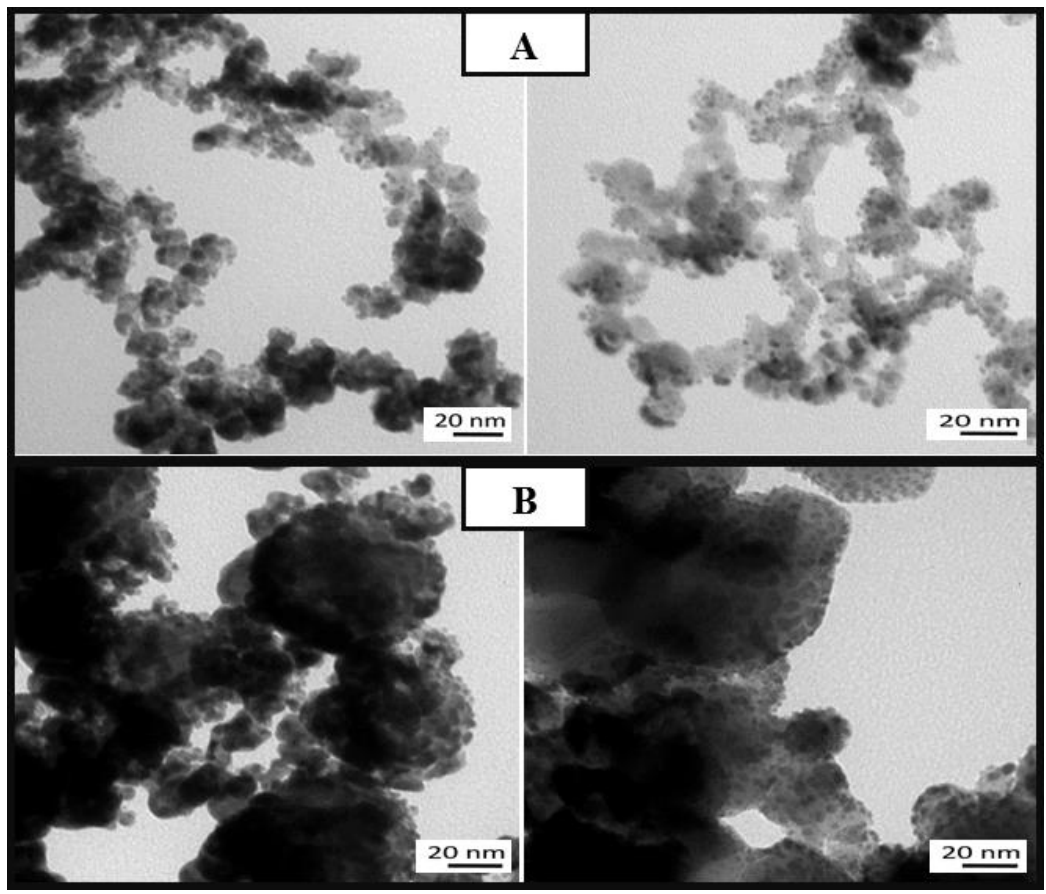

Figure 6.7: Variation in the degree of coverage and hybridisation of the core nano $\mathrm{TiO}_{\mathrm{x}}$ by $\mathrm{Au} / \mathrm{Pd}$ alloy, at different atomic \% concentrations of (A) $0.82 \% \mathrm{Au}, 0.30 \% \mathrm{Pd}$, and (B) $1.63 \% \mathrm{Au}, 0.68 \% \mathrm{Pd}$. 


\subsection{Changes in Absorbance due to Variations in Morphology and Phase Composition}

To comprehensively understand the optical absorption behaviour of the plasmon hybridised multiphase nano $\mathrm{TiO}_{x}$, three characteristic studies were performed. In the first study, the core composition of the nanomaterial was kept constant and only the extent of hybridisation by the plasmon metal nanoparticles on its surface was altered. This was done to study the effect of change in size and shape of the plasmon metal nanoparticles, on the surface plasmonic behaviour of the nanomaterial. In the second study, both the core composition of the core self-assembled nanomaterial as well as the extent of plasmon metal hybridisation on the nanomaterial were kept constant. The only variable that was altered was the average size of the individually aggregated nanospheres constituting the nano $\mathrm{TiO}_{\mathrm{x}}$ network. This was done to understand the change in the plasmonic coupling effect between the core nanomaterial and plasmon metal nanoparticles. Finally, a third study was then performed to investigate if a change in the core composition of the nanomaterial itself would influence its photon absorptive behaviour. In this final study, the extent of metal plasmon hybridisation on the nanomaterial network was kept constant.

Figure 6.8 shows the first study, where the variation in the absorption spectrum is seen for the core multiphase nano $\mathrm{TiO}_{\mathrm{x}}$ synthesised at a single laser processing condition of $1.64 \mathrm{~J} / \mathrm{cm}^{2}$ laser fluence and $1428 \mathrm{fs}$ pulse width. Thereby it represents having the same core material composition, but plasmon hybridised with different atomic percentage concentrations of both $\mathrm{Au}$ and $\mathrm{Au} / \mathrm{Pd}$ on its surface. Investigation of the material composition of this core synthesised nanomaterial, done in 
Chapter 5, had revealed that it was constituted of two common phases of $\mathrm{TiO}_{2}$ : anatase and rutile [115].
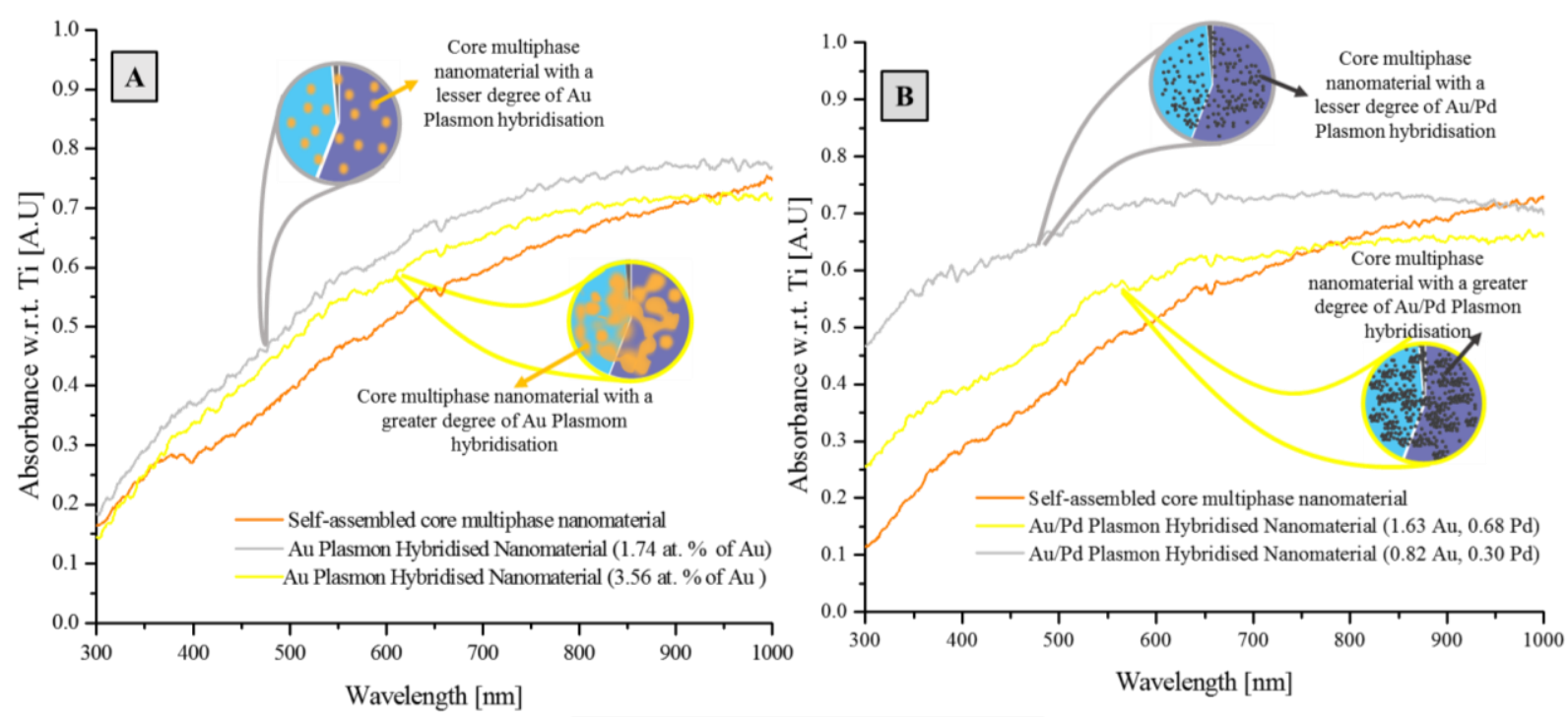

\begin{tabular}{|l|l|l|}
\hline Pie Chart Key: $\square$ Anatase $\square$ Rutile \\
\hline
\end{tabular}

Figure 6.8: Absorption spectrum of the core nano $\mathrm{TiO}_{\mathrm{x}}$ synthesised at $1.64 \mathrm{~J} / \mathrm{cm}^{2}$ laser fluence and $1428 \mathrm{fs}$ pulse width and having the same phase composition but plasmon hybridised with different atomic $\%$ concentrations of (A) $\mathrm{Au}$, and (B) Au/palladium alloy. The various colours in the pie chart schematic, representing an individually plasmon hybridised nanosphere constituting the nanomaterial network, indicate the various Titanium Oxide phases:

$$
\text { cyan - rutile, blue - anatase. }
$$

In Figure 6.8(A), a visible enhancement in absorption (almost 1.5 times that of core multiphase Titanium Oxide nanomaterial) is seen when the core nanomaterial is initially hybridised with just 1.74 atomic $\%$ concentration of $\mathrm{Au}$. This is due to the emergence of greater charge separation and plasmonic coupling effects between the smaller islands of $\mathrm{Au}$ and the core nanomaterial, which can lead to improved photocurrent density in the plasmon hybridised nanomaterial [105]. However, as the atomic \% concentration of $\mathrm{Au}$ is increased (and hence the fill factor) to 3.56, a drop in the absorption is seen. This drop in the absorption could be associated with greater surface 
recombination as well as photon reflection occurring at various possible sites on such larger and thicker islands of $\mathrm{Au}$. A similar observation was made for the nanomaterial sample, plasmon hybridised with $\mathrm{Au} / \mathrm{Pd}$, as seen in Figure 6.8(B), where the least amount of $\mathrm{Au} / \mathrm{Pd}$ hybridisation $(0.82 \% \mathrm{Au}, 0.30 \% \mathrm{Pd})$ gave the highest amount of photon absorption and further deposition led to decreased absorption. The obvious blue shift and broadening of the absorption spectrum, as observed for the Au/Pd Plasmon hybridised nanomaterial, is attributed to the smaller size of the plasmon $\mathrm{Au} / \mathrm{Pd}$ nanoparticles, as stated earlier.

Figure 6.9(A) and (B) show the spectral absorption of the nano $\mathrm{TiO}_{\mathrm{x}}$, plasma hybridised at a constant average atomic concentration of $\mathrm{Au}(1.74 \% \mathrm{Au})$ and $\mathrm{Au} / \mathrm{Pd}$ alloy $(0.82 \% \mathrm{Au}$, $0.30 \% \mathrm{Pd})$ respectively. The average size of the aggregated nanospheres constituting the $3 \mathrm{D}$ nanomaterial network was varied $(<5 \mathrm{~nm}$ difference) and the phase composition was kept constant (rutile and anatase), by synthesising them at different lasing conditions, as determined in Chapter 5. Smaller average sized nanospherical structures in the nanomaterial network were synthesised at laser processing conditions of $1.64 \mathrm{~J} / \mathrm{cm}^{2}$ laser fluence and $1428 \mathrm{fs}$ pulse width. Larger average sized nanospherical structures were synthesised at $1.64 \mathrm{~J} / \mathrm{cm}^{2}$ laser fluence and 214 fs pulse width. For the nano $\mathrm{TiO}_{\mathrm{x}}$, plasmon hybridised with $\mathrm{Au}$, as seen in Figure 6.9(A), nominal change in the absorption spectrum was observed with variation in the average size of the nanospheres. With $\mathrm{Au} / \mathrm{Pd}$ plasmon hybridised nanomaterial, a slightly broadened absorption spectrum in the visible region of the solar spectrum was observed only for the nanomaterial containing smaller sized aggregated nanospheres. This showed that the size of the nanospheres by themselves, which aggregate and self-assemble to form the core multiphase Titanium Oxide nanomaterial, did not have a significant influence on its photoabsorptive property. 

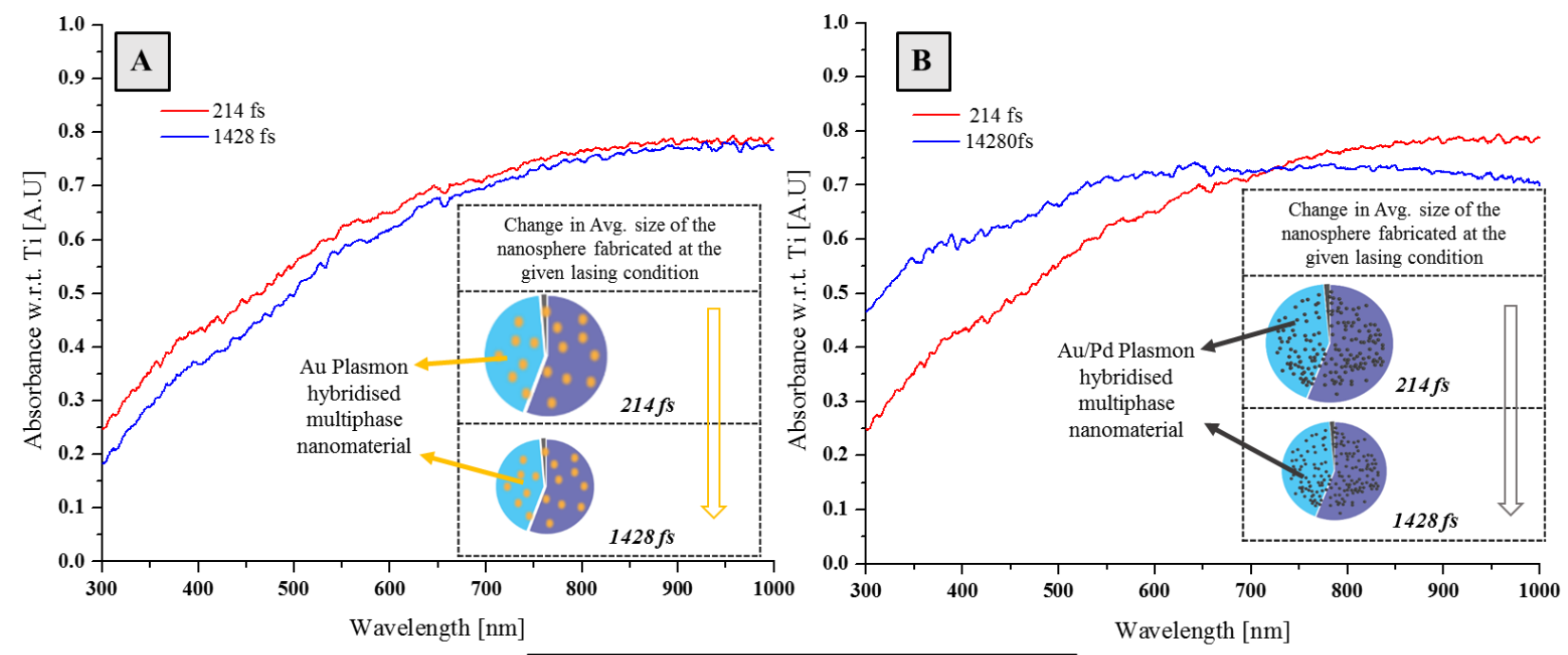

\begin{tabular}{|l|l|}
\hline Pie Chart Key: & Anatase \\
\hline
\end{tabular}

Figure 6.9: Absorption spectrum of the core nano $\mathrm{TiO}_{\mathrm{x}}$ synthesised at a laser fluence of $1.64 \mathrm{~J} / \mathrm{cm}^{2}$ and two pulse widths values (214 fs and $1428 \mathrm{fs}$ ), and plasmon hybridised with a constant atomic \% concentrations of (A) $\mathrm{Au}(1.74 \% \mathrm{Au})$, and (B) Au/palladium alloy (0.82\% Au, $0.30 \% \mathrm{Pd})$. The various colours in the pie chart schematic, representing an individually plasmon hybridised nanosphere constituting the nanomaterial network, indicate the various Titanium Oxide phases: cyan - rutile, blue - anatase.

Displayed in Figure 6.10, is the final study performed to examine if a change in phase composition of the core multiphase nanomaterial had any impact on its photon absorption ability. For this study, the phase composition of the nano $\mathrm{TiO}_{\mathrm{x}}$ was initially restricted to only two phases of Titanium Dioxide, anatase and rutile, by synthesising it at a laser fluence of $1.64 \mathrm{~J} / \mathrm{cm}^{2}$ and pulse width of 1428 fs as observed in section 5.2. A second sample of the core nanomaterial was then synthesised at a laser fluence of $5.14 \mathrm{~J} / \mathrm{cm}^{2}$ and pulse width of $1428 \mathrm{fs}$, to be composed of not two but multiple Oxide phases of Titanium, namely: rutile, anatase, $\mathrm{Ti}_{3} \mathrm{O}$ and non-stoichiometric (TiO.716)3.76, again seen in section 5.2. Both the nanomaterial samples were then plasmon hybridised with the same amount $\mathrm{Au}$ and $\mathrm{Au} / \mathrm{Pd}$ alloy and their absorption spectrum was acquired. 

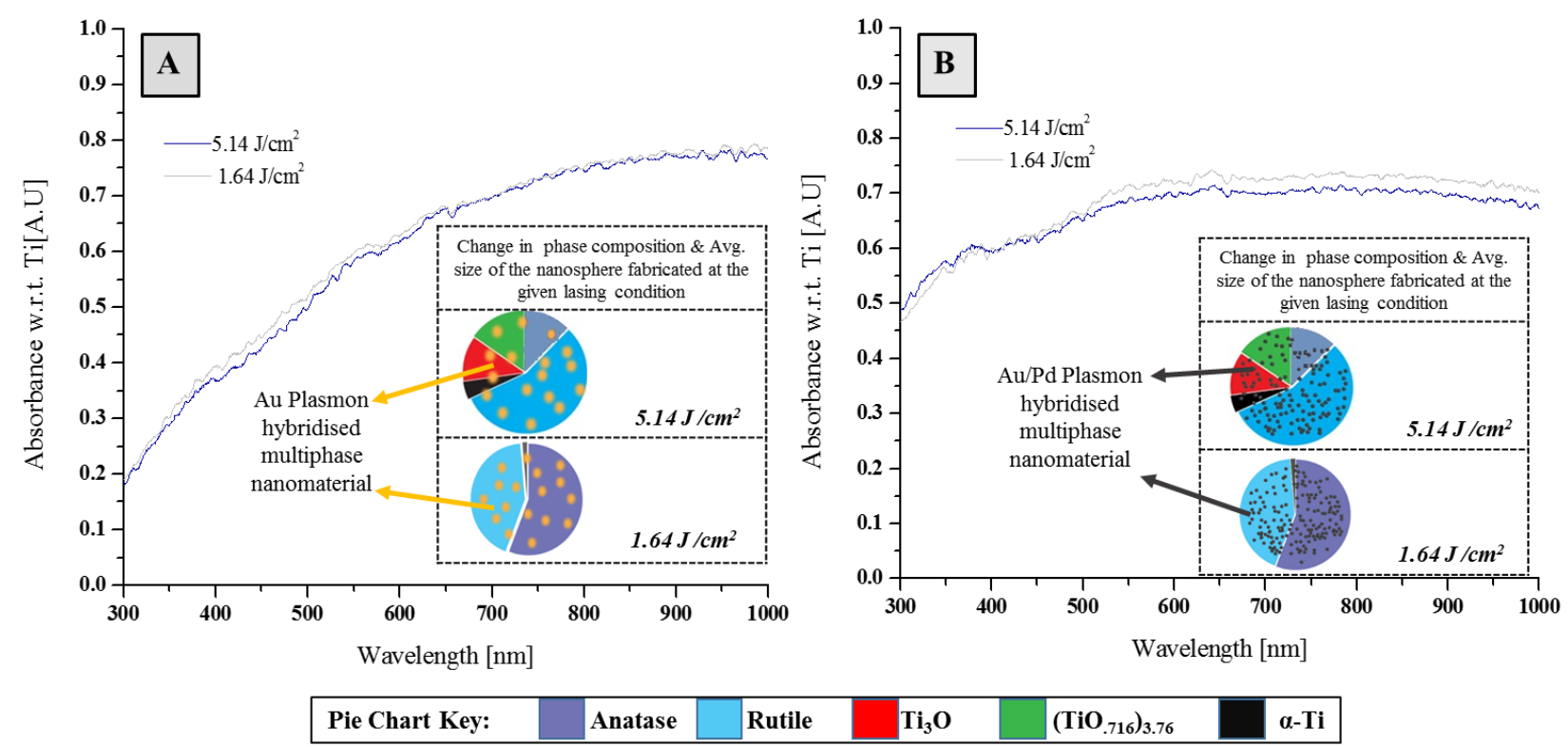

Figure 6.10: Absorption spectrum of the core self-assembled multiphase nano $\mathrm{TiO}_{\mathrm{x}}$ synthesised at a pulse width of $1428 \mathrm{fs}$ and two laser fluence values $\left(5.14 \mathrm{~J} / \mathrm{cm}^{2}\right.$ and $\left.1.64 \mathrm{~J} / \mathrm{cm}^{2}\right)$ and plasmon hybridised with a constant atomic \% concentrations of (A) $\mathrm{Au}(1.74 \% \mathrm{Au})$, and (B) Au/palladium alloy (0.82 \% Au, $0.30 \% \mathrm{Pd})$. The various colours in the pie chart schematic, representing an individually and plasmon hybridised nanosphere constituting the nanomaterial network, indicate the various Titanium Oxide phases: cyan - rutile, blue - anatase, black $-\alpha \mathrm{Ti}$, red $\mathrm{Ti}_{3} \mathrm{O}$, green- $\left(\mathrm{TiO}_{.716}\right)_{3.76}$.

As seen in Figure 6.10(A) and (B), the impact of variation in the phase composition of the core nanomaterial on the absorption spectrum was observed to be minimum. As such, after the abovementioned studies were conducted on the plasmon hybridised nanomaterial, it was inferred that only a change in the extent of hybridisation of the metal nanoparticles on the core multiphase Titanium Oxide nanomaterial was a factor significant enough, to influence its plasmonic behaviour and consequently its ability to absorb more photons over a broader range of the solar spectrum. 


\subsection{Summary}

In this chapter, we describe the plasmon hybridisation of the multiphase photoabsorptive nanomaterial reported earlier in Chapter 5. Further broadening of the absorption spectrum of the self-assembled nanomaterial over the entire visible and near infrared region of the solar spectrum was achieved via the induction of localised surface plasmonic behaviour through its hybridisation with $\mathrm{Au}$ and $\mathrm{Au} / \mathrm{Pd}$ alloy metal plasmons. An inclusive study conducted to understand the influence of various factors on the photon absorption ability of the Plasmon Hybridised multiphase nanomaterial revealed that the degree of hybridisation had a greater impact on the absorption capacity of the nanomaterial than the size and phase composition of the self-assembled nanospheres. This ensures greater controllability in being able to improve the optical behavior of the hybridised nanomaterial towards broadband wavelength absorption. Capitalization of the plasmonic resonance behaviour of $\mathrm{Au}$ and $\mathrm{Au} / \mathrm{Pd}$ metal plasmons in the visible region and the unique combination of absorption and scattering of deeper visible and near infrared photons, occurring throughout the self-assembled three-dimensional nanomaterial network lead to an overall absorption enhancement in both visible and near infrared region of the solar spectrum. 


\section{Chapter 7}

\section{Broadband Absorption Spectrum with}

\section{Disordered Multiphase Nano $\mathrm{TiO}_{\mathbf{x}}$}

\section{Networks}

The structure of $\mathrm{TiO}_{2}$ is known to be a function of its surface energy, which determines its reactive properties [122], [123]. Structure modification of $\mathrm{TiO}_{2}$ for altering its optical properties to better harvest light has been attempted by introducing lattice disorder and dopants [67]. As mentioned earlier in section 1.3, most recent efforts to enhance the optoelectronic properties of nanostructured $\mathrm{TiO}_{2}$ have focussed on 'self-doping', to introduce surface disorders and Oxygen vacancies in its structure without the use of external dopants [53]-[56]. They have resulted in the formation of colourful self-doped $\mathrm{TiO}_{2}$ which have gained a lot attention in the field of photovoltaics, especially 'black $\mathrm{TiO}_{2} ’$ [54], [66], [67].

This chapter introduces a unique disordered multiphase nano $\mathrm{TiO}_{\mathrm{x}}$, rich in Oxygen vacancies and defects. 'Self doping' by formation of high degree of vacancies was induced by tailoring its growth and phase state at the nano level. The plasma condensation mechanism was regulated and the kinetics of the plume was modified through the alteration of its ionic content. This was done by introducing Oxygen-rich and then Oxygen-poor conditions at the interface between the laser plasma plume and ambient atmosphere, alongside the alteration of interactions between the ultra- 
short laser pulses and the base Ti substrate. Through this, the desired ionisation process was stimulated and the plume temperature tuned. This enabled the self-assembly of the disordered multiphase nano $\mathrm{TiO}_{\mathrm{x}}$ to be managed at the molecular level and thereby engineer the defect-rich three-dimensional nano $\mathrm{TiO}_{\mathrm{x}}$ network with an enhanced and broadened photon absorption spectrum.

\subsection{Disordered Crystal Formation by Alteration of}

\section{Plume Kinetics}

For synthesising the disordered multiphase photo-absorptive nano $\mathrm{TiO}_{\mathrm{x}}$, laser intensity values ranging from $7.4 \times 10^{13}$ to $7.2 \times 10^{14} \mathrm{~W} / \mathrm{cm}^{2}$, and laser pulse width durations of $714 \mathrm{fs}$ and $1100 \mathrm{fs}$

were selected. The repetition rates selected were $4 \mathrm{MHz}, 8.36 \mathrm{Mhz}$ and $12.54 \mathrm{MHz}$. The laser beam was scanned across the surface of the Ti samples at a speed of $1 \mathrm{~mm} / \mathrm{s}$. Six tubes (masterflex 6411.14) were arranged in a square bracket, and were used to introduce Oxygen and Nitrogen gases, one at a time, which created Oxygen-rich and Oxygen-poor conditions respectively. This bracket was placed in parallel and in contact with the chuck on which the Ti samples had been mounted. This ensured that an uninterrupted and constant pressure gas was introduced at the site of laser interaction with the base Ti sample. All experiments were conducted at a constant gas flow rates of $4 \mathrm{SCFH}$ (standard cubic feet per hour).

The plume expansion dynamics plays an important role in the chemical composition and physical properties of the self-assembled three-dimensional network of multiphase nano $\mathrm{TiO}_{\mathrm{x}}$. Factors influencing these dynamics are the laser pulse intensity (energy per unit area per unit time) incident 
on the base Ti substrate given by equation 2 , the density of the metal vapour flux in the plasma plume, and the pressure exerted by the gas at the site of laser plume and ambient gas interaction.

The laser intensity in turn influences the average surface temperature reached on the Ti target and the plasma plume temperature [75], [91]. Furthermore, both the plume temperature and density affect the nucleation and growth of the nuclei and crystal, which eventually determines the physical and chemical aspects of the synthesised nanomaterial. This influence of the plume temperature and the density of ionic species on the nucleation and subsequent crystal formation of a nanomaterial has been elucidated earlier in section 2.3.

The effect of the plasma plume dynamic was taken advantage of to self dope and induce a higher degree of Oxygen vacancies and defects in nanostructures and thereby synthesise a disordered multiphase nano $\mathrm{TiO}_{\mathrm{x}}$. Oxygen vacancies are formed with ease under $\mathrm{O}$ poor conditions in Titanium Dioxide [124]. This is because, under such conditions, the low fermi energy levels cause a vacancy to form easily [124]. As such, to promote a higher degree of disorderliness in the nano $\mathrm{TiO}_{\mathrm{x}}$, poor Oxygen conditions were administered during material synthesis. To compare and demonstrate the effectiveness of the nano $\mathrm{TiO}_{\mathrm{x}}$ network in photon absorption when synthesised under poor Oxygen conditions, another set of samples of nano $\mathrm{TiO}_{\mathrm{x}}$ were created under Oxygen rich conditions, using similar laser processing parameters. The ionic content within the plume was thus manipulated prior to condensation and nucleation. This influenced the crystal growth after nuclei formation due to the regulation of cooling process and growth time, caused by the variation of Oxygen ion content in the plume. Oxygen rich conditions and Oxygen poor conditions were created by introducing Oxygen and Nitrogen gas at a constant flow rate around the laser plasma plume respectively. 
Under oxygen rich conditions, the higher concentration of oxygen atoms/molecules introduced around the laser-material interaction zone, ionise readily upon laser irradiation. As such, at a given laser intensity, large number of ionic species populate the plasma plume which start diffusing rapidly into the surrounding atmosphere to undergo secondary reactions with the oxygen atoms/molecules and their outward diffusion is therefore not restricted. This rapid diffusion of the plasma plume species influences the formation of the nanospheres in a major way. It results in higher cooling rate of the plasma plume conditions. This arrests the condensation and crystal growth of the nanospheres much earlier and causes the crystal structure formation to not be well defined.

On the contrary, when nitrogen gas is introduced for poor oxygen conditions, due to the high ionisation potential of nitrogen, its ions are not as readily formed as in case of oxygen. Also, in the short time scale of interaction between the ultrashort laser pulses and the target material, nitrogen doesn't take part extensively in the chemical reactions as the enthalpy of formation for TiN is much larger than the enthalpy of formation of $\mathrm{TiO}_{2}$, hence the reactions are dominated by Oxide formation [125]. Also, the Nitrides if present in low quantities would easily oxidise at the high temperatures. As such, the contribution of Nitrogen gas is mostly limited towards changing of plume kinetics. Due to this close to 'inert' behaviour of the nitrogen gas surrounding the laser plasma plume, the diffusion of the plume ionic species is restricted and the capping of the plasma plume heat takes place. As such, the plasma plume cools down at a slower rate, allowing longer time for condensation and crystal growth of the nanospheres to occur. This causes the structure of the nansospheres to be more crystalline. However, due to the absence of a high concentration of oxygen atoms/molecules within the plume, a higher degree of oxygen vacancies develop within the crystal structure. 
A graphical representation of how the Oxygen rich and Oxygen poor conditions affect the laser plasma plume formation and thus the synthesis of nano $\mathrm{TiO}_{\mathrm{x}}$ is shown in Figure 7.1.

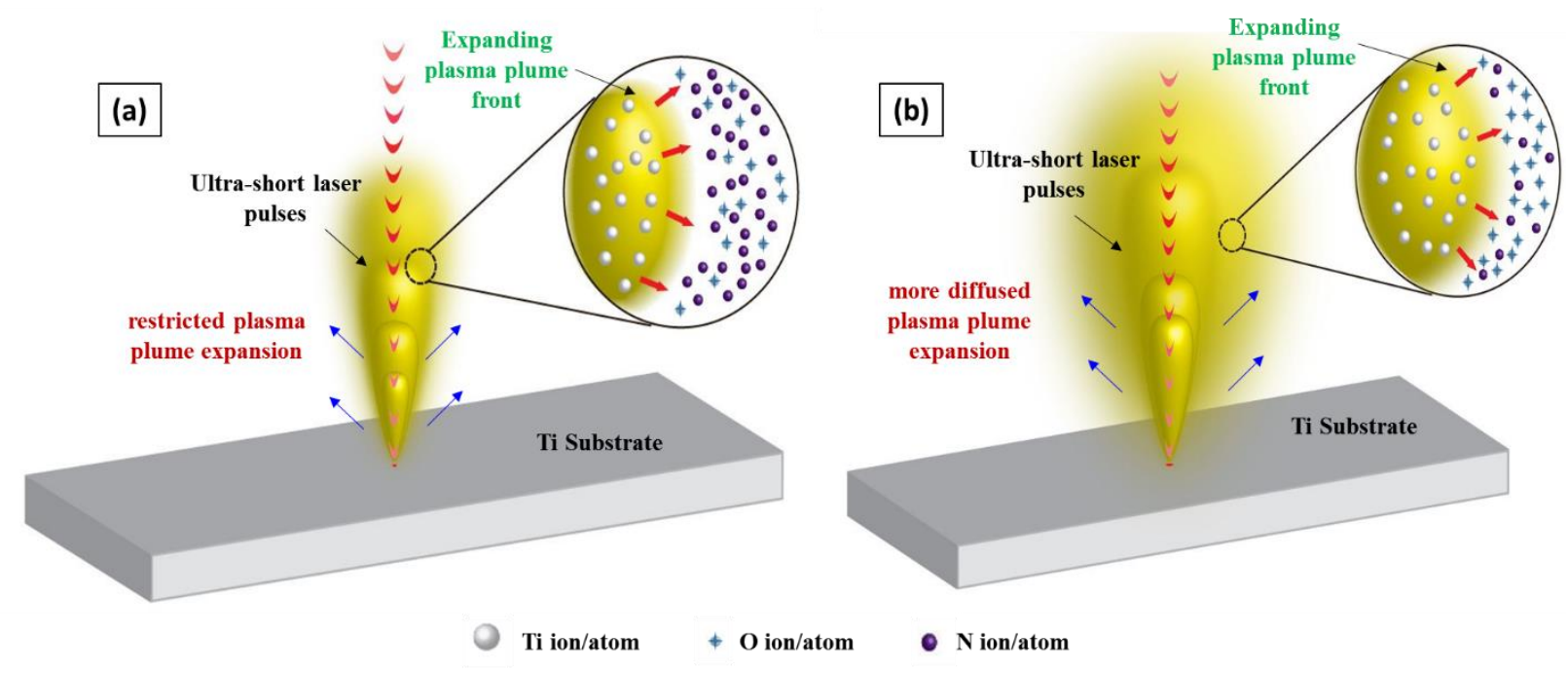

Figure 7.1: Graphical representation of the synthesis of nano $\mathrm{TiO}_{\mathrm{x}}$ under (a) poor Oxygen, and (b) Oxygen rich conditions, with the variation in the expansion of the laser plume and the ionic content around it.

\subsection{Surface and Material Characterization of}

\section{Disordered Multiphase Nano $\mathrm{TiO}_{\mathrm{x}}$}

The surface morphology of the self-assembled nano $\mathrm{TiO}_{\mathrm{x}}$ synthesised under both Oxygen rich and Oxygen poor conditions was first observed by using FE-SEM. A network consisting of coalesced and aggregated nanospheres like structures was seen. These nanospheres were seen to further coalesce to form fiber like structures which finally formed layers of 'circular pore', that were arranged over each other to a three-dimensional network. Earlier studies done have shown that the density of such a network diminished under lower laser processing intensities [115]. At a constant laser intensity, the morphology of the nanomaterial showed similar formation of circular pores and 
three-dimensional network at both Oxygen rich and Oxygen poor conditions. However, upon the inspection of the individual nanospheres comprising the nano network, it was observed that the nano $\mathrm{TiO}_{x}$ synthesised under poor Oxygen conditions had a much denser network. Furthermore, a wider size distribution of nanospsheres was seen and on an average, they appeared larger. Figure 7.2(a) and (b) show this variation in the synthesised nano $\mathrm{TiO}_{\mathrm{x}}$ under Oxygen rich and Oxygen poor conditions respectively.
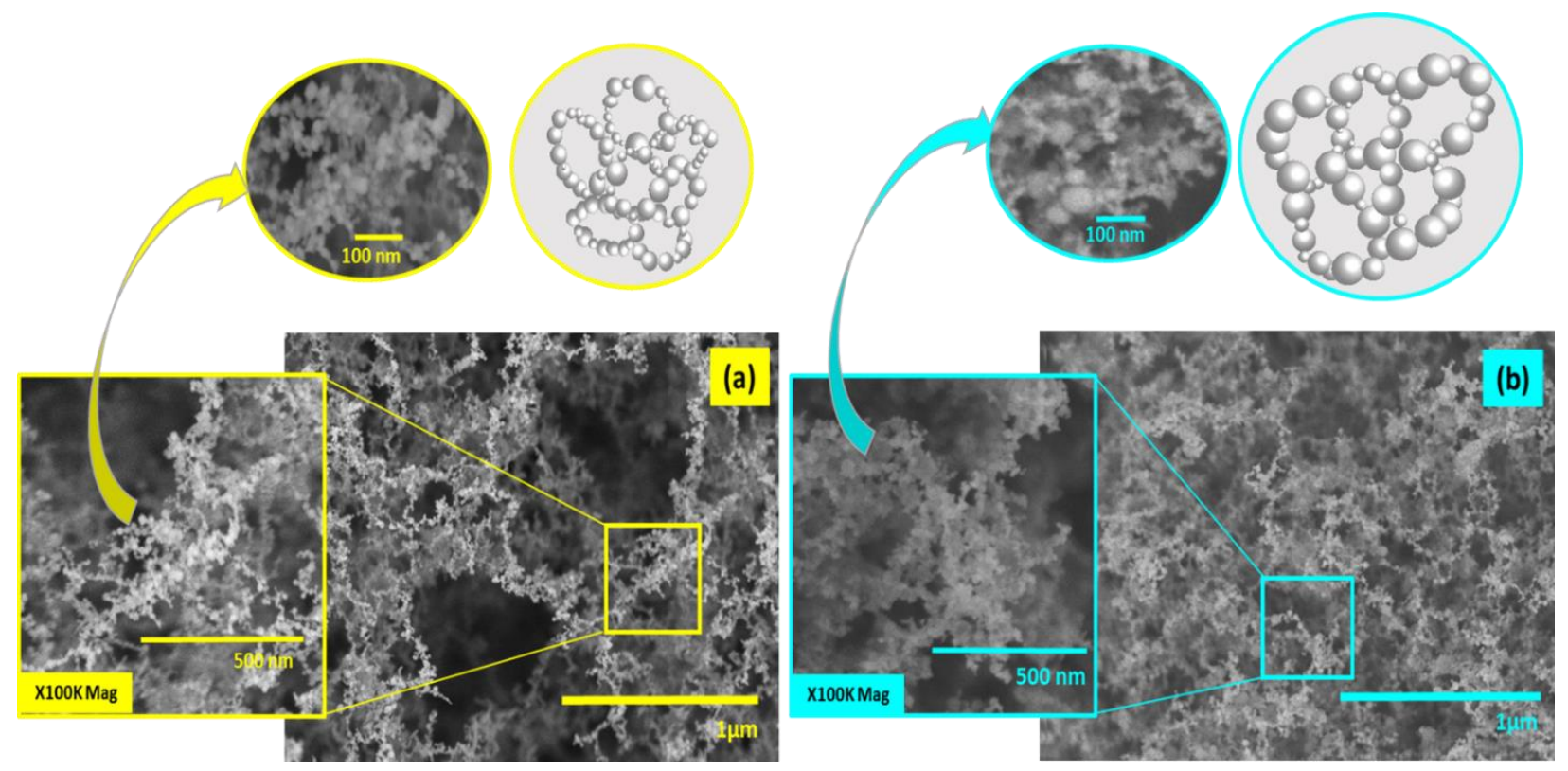

Figure 7.2: FE-SEM images of the 3D self assembled network of nano TiOx synthesised under (a) Oxygen rich, and (b) Oxygen poor conditions. Magnified images show the network being comprised of nanospheres self-assembled to form the structure.

The surface characterisation of the photo absorptive nano $\mathrm{TiO}_{\mathrm{x}}$ network was performed using an XPS to determine the change in chemical composition and the oxidation states of Titanium and Oxygen, when synthesised in an Oxygen ion rich and poor conditions. The Ti $2 p$ spectra of all the material samples, had two peaks corresponding to Ti $2 \mathrm{p}_{1 / 2}$ and $\mathrm{Ti} 2 \mathrm{p}_{3 / 2}$. The peaks centered on $458.48 \mathrm{eV}$ and $464.28 \mathrm{eV}$ respectively, for the nano $\mathrm{TiO}_{\mathrm{x}}$ synthesised under Oxygen poor 
conditions but shifted slightly to higher binding energy centred on $458.68 \mathrm{eV}$ and $464.48 \mathrm{eV}$ for the nano material synthesised in an enriched Oxygen ion environment. Full width half maximum of all the Ti $2 \mathrm{p}$ peaks was $2.1 \pm 0.1 \mathrm{eV}$ and peak separation between $2 \mathrm{p} 1 / 1$ and $2 \mathrm{p} 3 / 2$ was $5.8 \mathrm{eV}$. In $\mathrm{TiO}_{2}$, the standard binding energy of $\mathrm{Ti} 2 \mathrm{p}_{3 / 2}$ for $\mathrm{Ti}^{4+}$ is $459.5 \mathrm{eV}$ [126]. The lower binding energies observed for this peak in the nano $\mathrm{TiO}_{\mathrm{x}}$ are thus attributed to $\mathrm{Ti}^{3+}$.

Furthermore, the $\mathrm{O}$ s1 peak was shifted to a lower binding energy from $530.1 \mathrm{eV}$ to $529.9 \mathrm{eV}$ for the nano TiOx synthesised under Oxygen poor conditions, when compared to the one synthesised under rich Oxygen conditions. This indicated an obvious decrease in the $\mathrm{Ti}^{4+}$ concentration for the nano $\mathrm{TiO}_{\mathrm{x}}$ synthesised under poor Oxygen conditions. It also suggested the probability of higher concentration of defects being formed under such conditions. Furthermore, shoulder peak for O s1 spectra was observed around $531.5 \mathrm{eV}$, for the nano $\mathrm{TiO}_{\mathrm{x}}$ synthesised at both Oxygen rich and Oxygen poor conditions, which corresponds to the Oxygen vacancy $\mathrm{Ti}^{3+}$ state [127]. This further indicated the presence of disorders and defects in the synthesised nano $\mathrm{TiO}_{\mathrm{x}}$. The valance band (VB) plots of the XPS survey were also observed to be more lifted than the valance band of $\mathrm{TiO}_{2}$ $(2.95 \mathrm{eV})$, averaging around $2.5 \mathrm{eV}$, as seen in Figure 7.3(c). An additional slope seen on the VB spectrum around $1.8 \mathrm{eV}$ also denoted the presence of defects and vacancies, which are known to generate such valance band tails [67]. The Ti $2 p$ spectra and the $\mathrm{O}$ s1 spectra of nano $\mathrm{TiO}_{\mathrm{x}}$ synthesised under Oxygen rich and Oxygen poor conditions have been plotted and shown in Figure 7.3(a) and (b) respectively. The VB spectra shown in Figure 7.3(c) has marked slopes indicating the average valence band. 

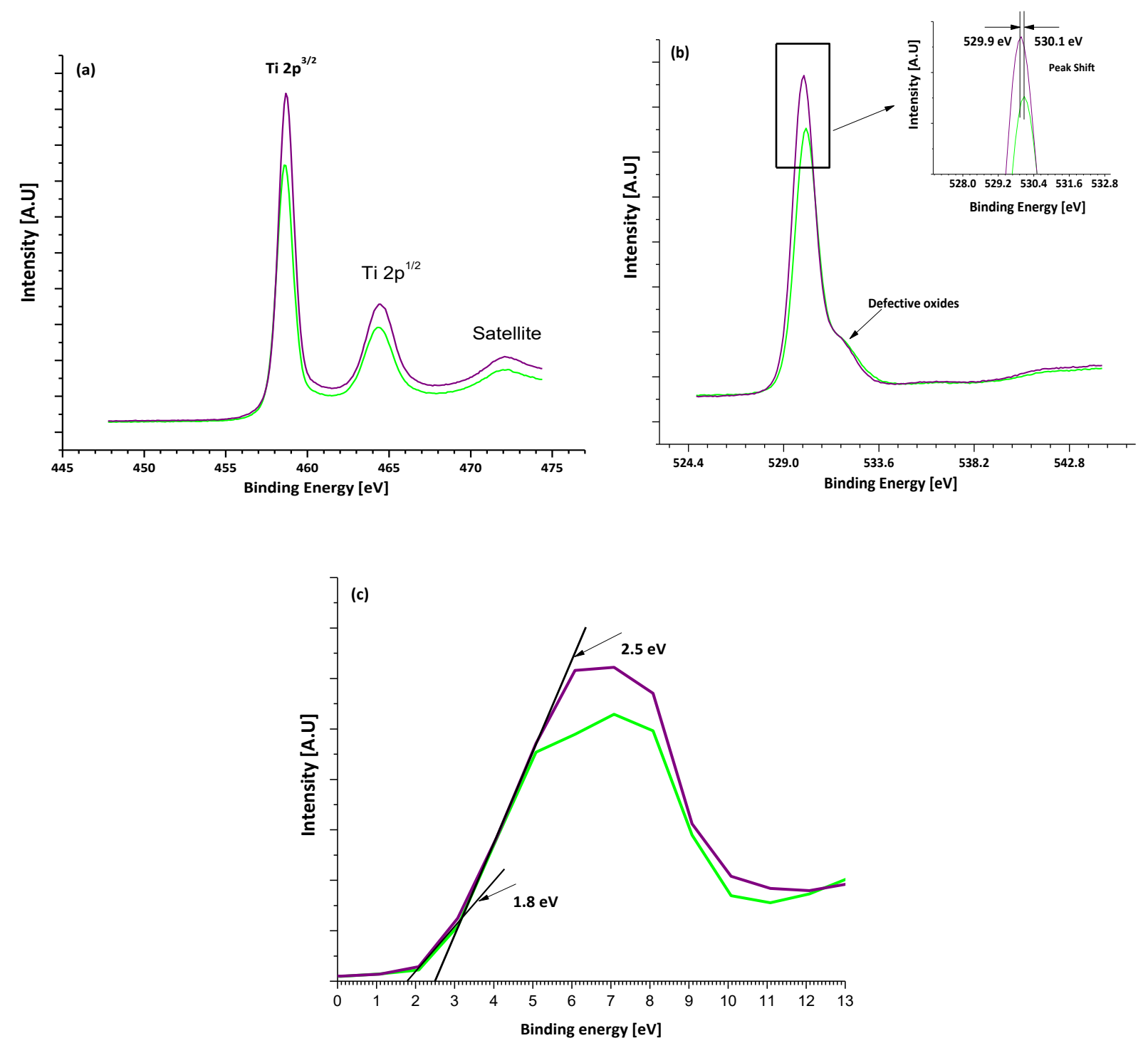

Figure 7.3: XPS spectra of the nano Titanium. (a) Ti 2p spectra, (b) O s1 spectra, and (c) Valence band (VB) spectra. Purple indicates the spectra of the nano $\mathrm{TiO}_{\mathrm{x}}$ synthesised under Oxygen poor conditions and a laser intensity of $7.2 \times 10^{14} \mathrm{~W} / \mathrm{cm}^{2}$. Green colour indicates the spectra of the nano $\mathrm{TiO}_{\mathrm{x}}$ synthesised under Oxygen rich conditions and a laser intensity of $1.4 \times 10^{14} \mathrm{~W} / \mathrm{cm}^{2}$. Inset in (b) shows the shift in binding energy and the slopes marked in (c) indicate the valance band values.

Further material and phase characterisation of the nano $\mathrm{TiO}_{\mathrm{x}}$ was performed using an $\mathrm{XRD}$ as the depth of study of the XPS technique is often limited to a couple of nanometers due to it being a highly surface sensitive technique. Reitveld fitting of the acquired XRD patterns found the 
approximate quantification of the phase constituents of the synthesised nano $\mathrm{TiO}_{\mathrm{x}}$. The source of the X-rays for XRD was CuK $\alpha$ radiation of $1.54184 \AA$ wavelength. The $2 \theta$ value for each of the XRD profile ranged from $30-78^{\circ}$. Compositional analysis of the acquired XRD pattern was done using the Search-Match ${ }^{\mathrm{TM}}$ procedure. The presence of sharp peaks on the XRD pattern indicated that the synthesised nanomaterial was crystalline in nature. Peaks corresponding to Titanium, rutile, anatase, $\mathrm{Ti}_{3} \mathrm{O}, \mathrm{Ti}_{3} \mathrm{O}_{5}$ and a non-stoichiometric Oxide of Titanium $\left(\mathrm{TiO}_{.316}\right)_{7.36}$ were seen. This indicated that the nano $\mathrm{TiO}_{\mathrm{x}}$ consisted of multiple oxidation states and phases. The absence of distinct peaks corresponding to these lower Oxide phases of Titanium in the previous XPS study might indicate that the stoichiometric change to such oxide phases of Titanium occurred at greater depths not within the range scanned by an XPS. Quantitative analysis revealed that the dominant phase in all samples was rutile, with the nano $\mathrm{TiO}_{\mathrm{x}}$ synthesised under Oxygen rich conditions having a greater weight percentage of it. Furthermore, the nanomaterial synthesised under poor oxygen conditions exhibited higher amounts of the lower oxidation state Oxide phases of Titanium, namely $\left(\mathrm{TiO}_{316}\right)_{7.36}, \mathrm{Ti}_{3} \mathrm{O}$ and $\mathrm{Ti}_{3} \mathrm{O}_{5}$. Anatase, the more active phase of $\mathrm{TiO}_{2}$ was found to exist in the nano $\mathrm{TiO}_{\mathrm{x}}$ formed under poor Oxygen conditions and at a lower laser intensity. This could be due to that fact that anatase is more stable in nano crystalline form under lower temperature and transforms to rutile as higher temperature are reached [128]. In addition, minor traces of Titanium Nitride (TiN) were found in the nanomaterial synthesised under poor Oxygen conditions with the reactive Nitrogen shield surrounding the laser plasma plume. As stated earlier, the reason for only slight traces of TiN seen could be due its oxidation under high temperatures and that the fact that the Oxide formation is more favoured due to its lower enthalpy of formation than the TiN reaction [125]. 
A closer look at the nature of the XRD peaks showed that they were less sharp and slightly broader for the nano $\mathrm{TiO}_{\mathrm{x}}$ formed under Oxygen poor conditions. This indicated that there were indeed greater Oxygen defects formed under such conditions. The obvious increase in the lower oxidation states and Oxygen defects in the nano $\mathrm{TiO}_{\mathrm{x}}$ under poor Oxygen conditions was thus evidenced by the XRD study. Figure 7.4 shows the XRD patterns obtained for the nano $\mathrm{TiO}_{\mathrm{x}}$ synthesised at various conditions. The crystallite size was calculated using the Scherrer equation [129], [130]:

$$
D_{\mathrm{p}}=\frac{0.94 \lambda}{\beta \cos \theta}
$$

where $D_{\mathrm{p}}$ is the average crystallite size, $\beta$ is the line broadening in radians at half the maximum intensity (FWHM), $\theta$ is the Bragg angle and $\lambda$ is the X-ray wavelength. The average crystallite size was calculated for all the major peaks with highest intensities for all the phases. The crystallite size of rutile corresponding to three highest intensity peaks at $2 \theta$ values of 27.4, 54.3 and 56.5 were found to be $57.89 \mathrm{~nm}, 37.93 \mathrm{~nm}$ and $31.93 \mathrm{~nm}$ respectively, whereas the crystallite size of anatase for its highest intensity peaks at $25.275^{\circ}$ was $15.46 \mathrm{~nm}$. This corresponds well with literature that rutile is more stable at larger particle sizes [22]. The crystallite size of $\mathrm{Ti}_{3} \mathrm{O}$ corresponding to its highest intensity peaks at $2 \theta$ value of 37.1 and 40.125 was calculated to be $10.94 \mathrm{~nm}$ and $19.64 \mathrm{~nm}$ respectively. Similarly, the crystallite size of the non-stoichiometric Oxide ( $\left.\mathrm{TiO}_{.316}\right)_{7.36}$ for one of its highest peaks at $2 \theta$ value of 42.3 was $11.8 \mathrm{~nm}$. Due to the only minor traces of $\mathrm{Ti}_{3} \mathrm{O}_{5}$ and TiN being present and existence of noise in the diffraction patterns, their crystallite size could not be calculated. It is worthwhile to keep in mind that the Scherrer equation is seldom indicative of the accurate value of the crystallite size because a number of aspects, which also include instrumentation factors, can influence the width of the XRD peaks. However, it can help give an approximate estimation of the crystallite size. Furthermore, the presence of defects 
and faults in the structure, as evident in our nano $\mathrm{TiO}_{\mathrm{x}}$, can also result in peak broadening, resulting in erroneous crystallite size values. Peak splitting and the presence of peak shoulders was observed for some of the peaks in the acquired XRD patterns after peak fitting. Figure 7.4 shows some of the areas magnified where such peaks were found. This is indicative of secondary phase transitions and defects. To gather further evidence about the degree of crystallinity, nature of Oxygen defects and the size of the nanospheres, HR-TEM analysis was performed.

To determine the size distribution of the individual spherical nanostructures constituting the nano $\mathrm{TiO}_{\mathrm{x}}$ and obtain their atomic lattice image, the synthesised nanomaterial was analysed using HR-TEM. The instrument used was JOEL JEM 2010 and the samples were placed on a copper grid for examination. Image ${ }^{\mathrm{TM}}$ was used as a post-processing tool for taking measurements of the HRTEM images. Figure 7.5 shows the size distribution of the nanospheres synthesised under Oxygen rich and Oxygen poor conditions, at varying laser intensities, with their corresponding HR-TEM micrographs given as inset. The mean nanosphere size detected at each condition is also shown on the size distribution plots. 


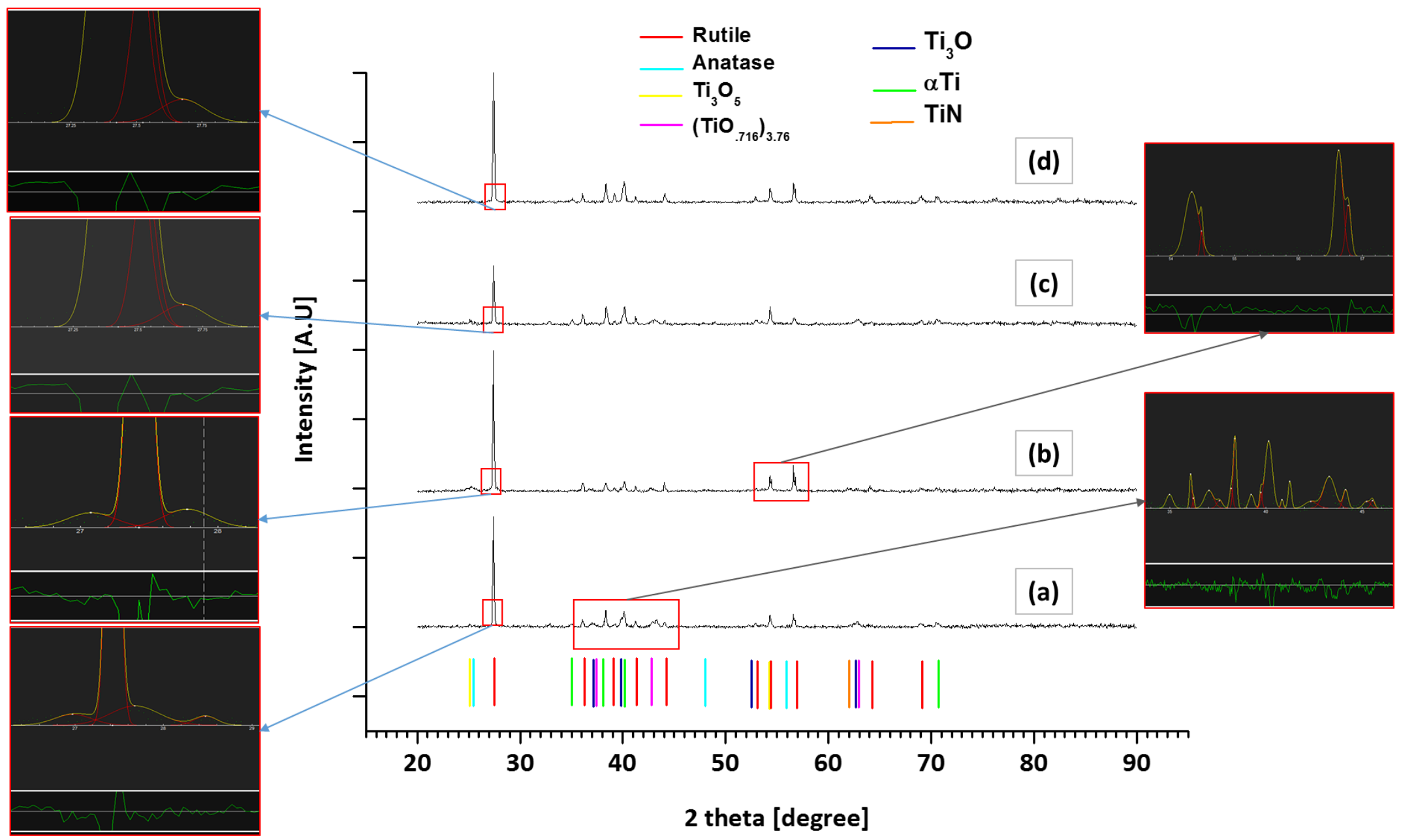

Figure 7.4: XRD pattern of the nano $\mathrm{TiO}_{\mathrm{x}}$ synthesised under Oxygen poor conditions and a laser intensity of (a) $1.4 \times 10^{14} \mathrm{~W} / \mathrm{cm}^{2}$ and (b) $7.2 \times 10^{14} \mathrm{~W} / \mathrm{cm}^{2}$, and under Oxygen rich conditions and a laser intensity of (c) $1.4 \times 10^{14} \mathrm{~W} / \mathrm{cm}^{2}$ and (d) $7.2 \times 10^{14} \mathrm{~W} / \mathrm{cm}^{2}$. Different coloured bars at the bottom of the plot represent the reference XRD peaks from PDF-2 and ICSD database corresponding to each of the phase constituents. A few of the areas of the XRD pattern where peak broadening and splitting was observed has been magnified and shown. 
As observed, all the nanosphere size distributions were unimodal in nature. This is in contrast to the typical bimodal distribution seen for femtosecond laser ablation process, where both ionised and neutral species influence the formation of nanostructures [131]-[133]. Narrow unimodal distributions are generally observed for nanosecond laser ablation where longer pulse durations cause additional excitation of the plume causing the plume content to be uniform [131]. The presence of such a unimodal distribution of nanospheres in the nano $\mathrm{TiO}_{\mathrm{x}}$ network might indicate that their growth was influenced by a similar excitation brought about by the ionised species present around the laser plasma plume, causing the change in plume kinetics. The nano $\mathrm{TiO}_{\mathrm{x}}$ synthesised under Oxygen rich conditions showed a narrow size distribution, ranging on an average from one to $30 \mathrm{~nm}$. A wider size distribution was observed for the nano $\mathrm{TiO}_{\mathrm{x}}$ synthesised under poor Oxygen conditions, which ranged on an average from one to $70 \mathrm{~nm}$, depending on the laser processing conditions at which it was formed. In the presence of Oxygen rich species in the laser plasma plume, almost a fifty percent reduction in the mean size of the nanospheres was observed at laser intensity conditions at which it was synthesised. This can be seen in Figure 7.6.In addition, an upward trend in nanosphere growth is seen with increasing laser intensity. This conforms well to previous studies where an increase in nanosphere size was seen with increasing laser intensity [115], [133]. Additionally, the mode value of the size distribution curve drawn showed that a greater number of large sized nanospheres were present in the nano $\mathrm{TiO}_{\mathrm{x}}$ formed under poor Oxygen conditions. A possible reason for this size variation could be explained by the fact that the time required for diffusion of the laser plasma plume into the ambient atmosphere is a major influencing factor determining it. Under Oxygen rich conditions, as described earlier in section 7.1, initially, larger concentration of the ionised species starts building up in the laser plasma plume as the ionisation potential of oxygen is less. These ionic species diffuse faster into 
the surrounding oxygen rich atmosphere to undergo further reactions with the oxygen species. This results in fewer interactive collisions between the ionic species within the laser plasma plume, causing lower coalescence and aggregation of the nuclei leading to a restricted particle growth. On the other hand, the poor Oxygen conditions generated by introducing Nitrogen gas around the laser plasma plume result in reduced ionised species in the plume due to the higher ionisation potential of nitrogen. However, the presence of such large number of Nitrogen atoms/molecules, which have a larger ionisation potential, cause them to exhibit an almost inert behaviour. This caps and restricts the ionised species within the plasma plume causing their diffusion into the surrounding atmosphere to be largely diminished. As such large number of collisions occur, which lead to more coalescence and aggregation causing larger nanospheres to be formed. Furthermore, the lower cooling rate as a result of capping of the plasma plume causes nucleation and condensation to last longer. This causes larger nanospheres to be formed with a wider size distribution. This wide distribution seen in the nano $\mathrm{TiO}_{\mathrm{x}}$ is an essential factor which will help achieve a broadened absorption spectrum and as we will observe later. 

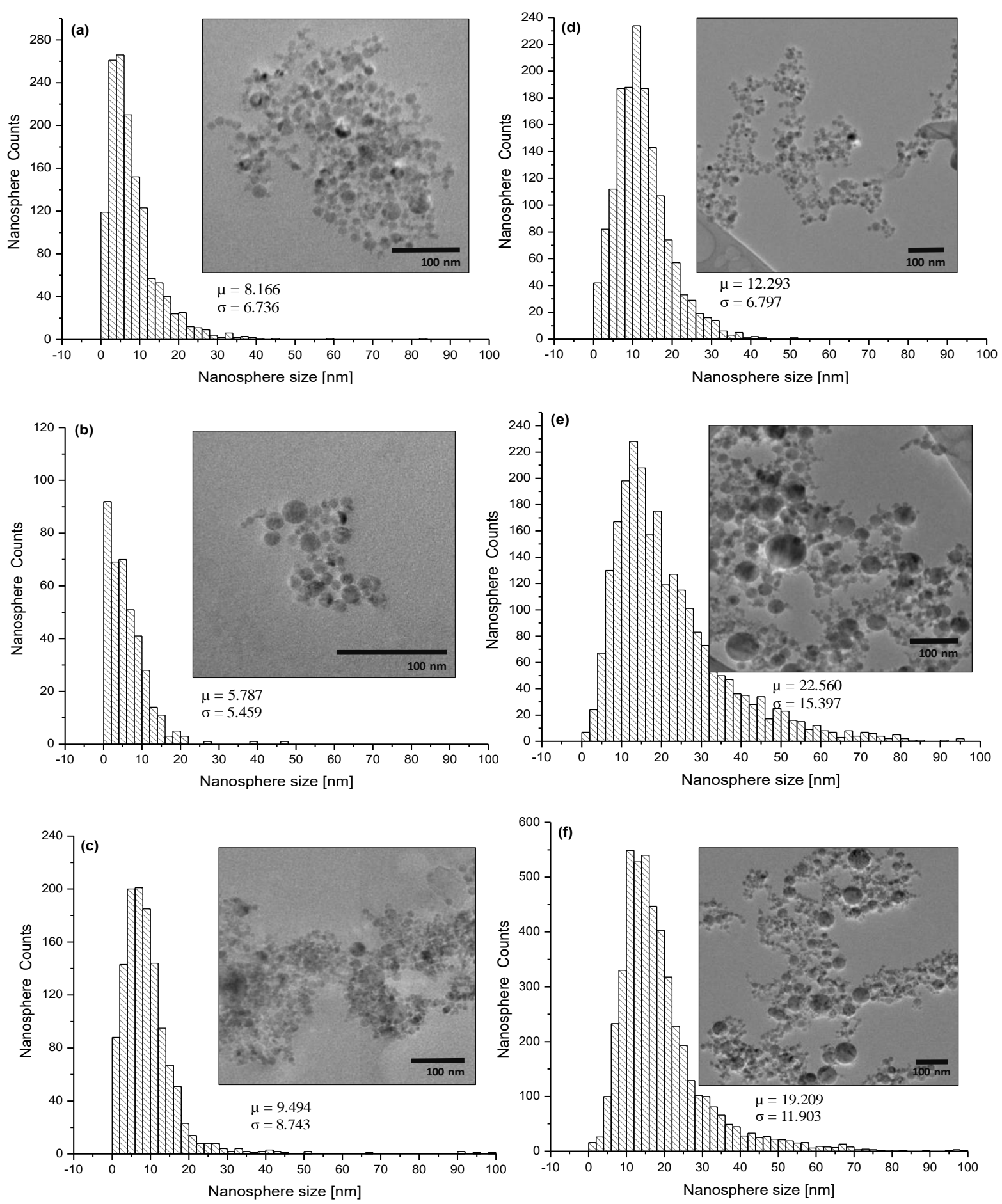

Figure 7.5: Size distribution of the individual nanospheres constituting the network of nano $\mathrm{TiO}_{\mathrm{x}}$ synthesised at different laser intensities and under Oxygen rich (a) $2.2 \times 10^{14} \mathrm{~W} / \mathrm{cm}^{2}$, (b) $4.6 \times 10^{14} \mathrm{~W} / \mathrm{cm}^{2}$ and (c)

$7.2 \times 10^{14} \mathrm{~W} / \mathrm{cm}^{2}$, and Oxygen poor conditions (d) $1.1 \times 10^{14} \mathrm{~W} / \mathrm{cm}^{2}$, (e) $4.6 \times 10^{14} \mathrm{~W} / \mathrm{cm}^{2}$ and (f) $7.2 \times 10^{14} \mathrm{~W} / \mathrm{cm}^{2}$. Inset are the corresponding TEM images. 


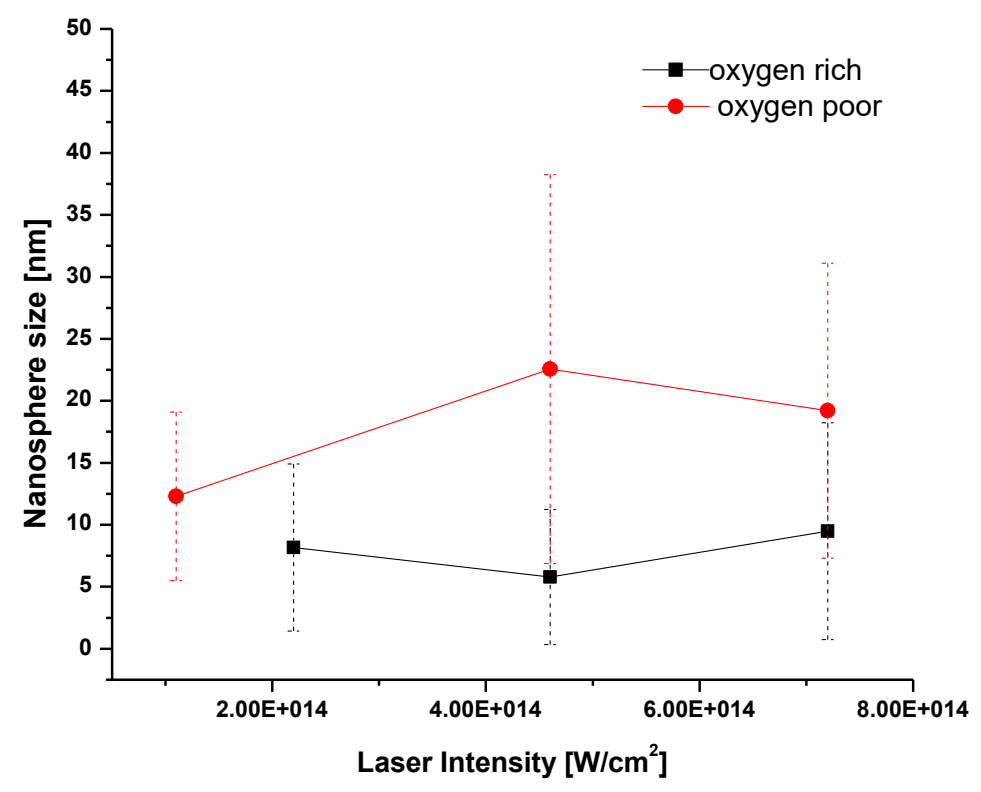

Figure 7.6: Variation of mean nanosphere size with laser intensity, as seen in the distribution plots for both Oxygen rich and Oxygen poor conditions. Errors bars indicate the standard deviation.

\subsection{Analysis of Defect Types and Densities}

The atomic lattice spacing and the disorders present in the nano $\mathrm{TiO}_{\mathrm{x}}$ were first studied by locally examining the HR-TEM images. As it was revealed earlier in the XRD study and HR-TEM images, the samples were crystalline in nature and had a spherical morphology. It was observed that the nanospheres synthesised under poor Oxygen conditions were more crystalline when compared to the nano material synthesised under Oxygen rich conditions and as such their lattice fringes were more clear and well defined. This difference between the Oxygen rich and Oxygen poor synthesised nano $\mathrm{TiO}_{\mathrm{x}}$ can be seen in Figure 7.7(a) and (b). 

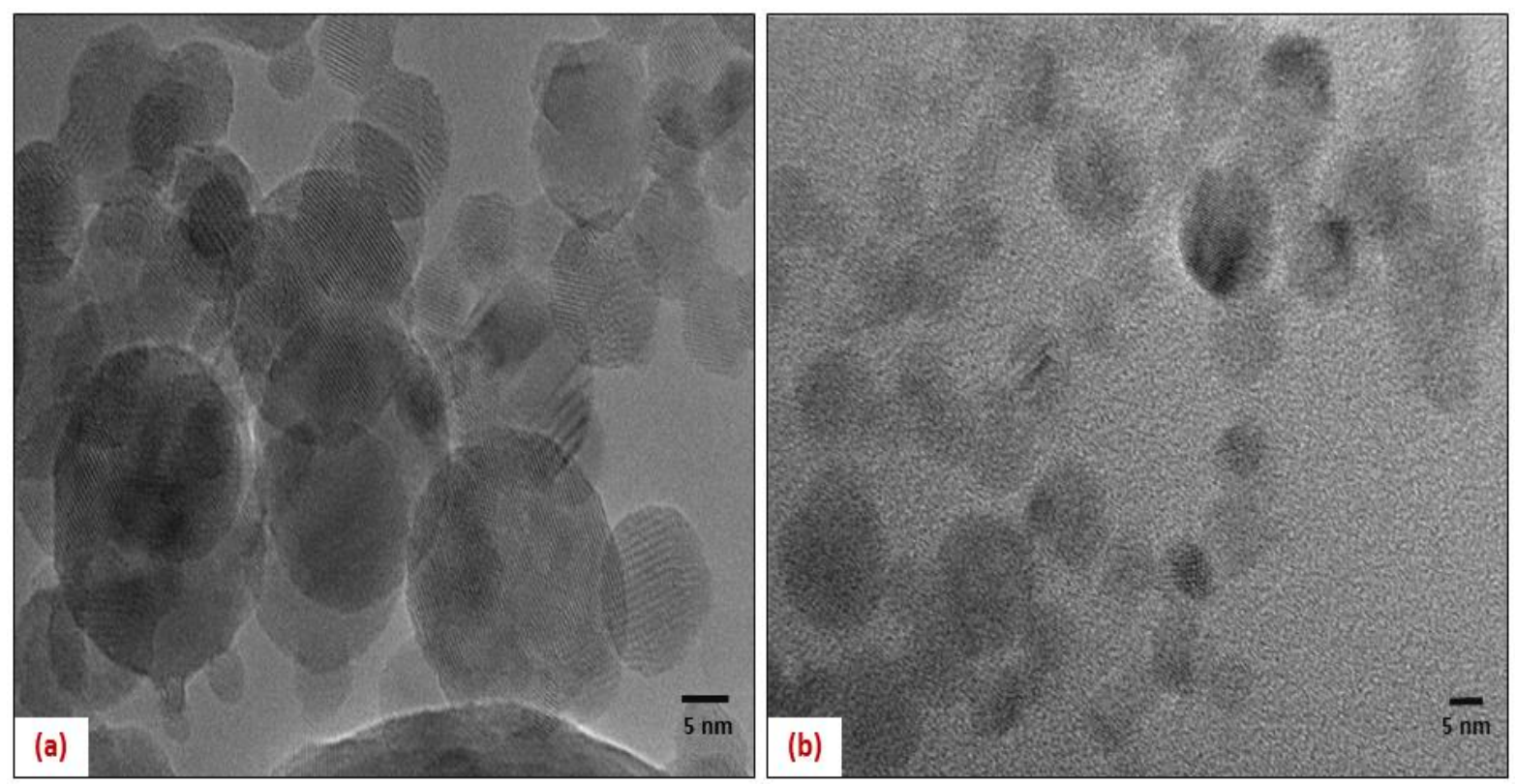

Figure 7.7: HR- TEM image of the nano TiOx showing higher degree of crystallinity and defects in the nanospheres when synthesised under (a) poor Oxygen conditions than at (b) Oxygen rich conditions.

Upon closer examination of single nanospheres, numerous lattice orientations and a number of random disorders and vacancies were observed. These defects were greater in density in the crystal lattices of the nano $\mathrm{TiO}_{\mathrm{x}}$ synthesised under poor Oxygen conditions. Oxygen vacancies to a higher degree and stacking defects were also seen, along with super lattices where the crystal displayed highly ordered structure. All the point and extended defects, calculated d-spacing value of the lattice fringes, along with areas where lattice mismatch and multiple lattice orientations were seen, have been shown and marked in Figure 7.8(a)-(h).

Extended defects like stacking faults (Figure 7.8(b), (e) and (h)) and point defects due to Oxygen vacancies (Figure 7.8(c), (d), (f), (g) and (h)) were seen. Furthermore, lattice mismatching (Figure 7.8(a), (d) and (h)) within a single nanosphere was observed, which proved that these nanospheres were truly multiphase in nature. The d-spacing of few of the lattice fringes clearly seen have been marked and their FFT images have been given as inset. These values of d-spacing were found to 
slightly larger than the standard values found in the ICSD database. For example, the highest intensity peak of the XRD pattern acquired earlier corresponded to the $\left[\begin{array}{lll}1 & 1 & 0\end{array}\right]$ plane of rutile at $27.434^{\circ}$. The standard lattice d-spacing for this plane is $3.24845 \AA$. However most of the measured lattice spacing was found to be between 3.5-3.9 $\AA$. This means that the disordered nanomaterial has a tensile strain.

The HR-TEM analysis thus confirmed that better-defined crystals with a greater density of defects were formed under poor Oxygen conditions by introducing Nitrogen gas. As discussed earlier, the less amount of the easily ionisable Oxygen atoms/molecules around the laser plume resulted in reduced ionisation within the plume thus provided an effect akin to shielding which restricted the diffusion of the plasma plume into the surrounding medium. The restricted diffusion resulted in longer condensation to form larger particles and to a lower cooling process which caused the nucleation of well-defined crystal nano structures. Furthermore, fewer oxygen atoms/molecules created reducing conditions leading to higher degree of defects and vacancies. As such, for clarity, the nano $\mathrm{TiO}_{\mathrm{x}}$ synthesised under poor Oxygen conditions will be labelled as $\mathrm{HDD}$-nano $\mathrm{TiO}_{\mathrm{x}}$, for 'high Density Defect nano $\mathrm{TiO}_{\mathrm{x}}$ ' and the one synthesised under Oxygen rich conditions will be labelled as LDD-nano $\mathrm{TiO}_{\mathrm{x}}$, for 'Low Density Defect nano Titanium Oxide'. 

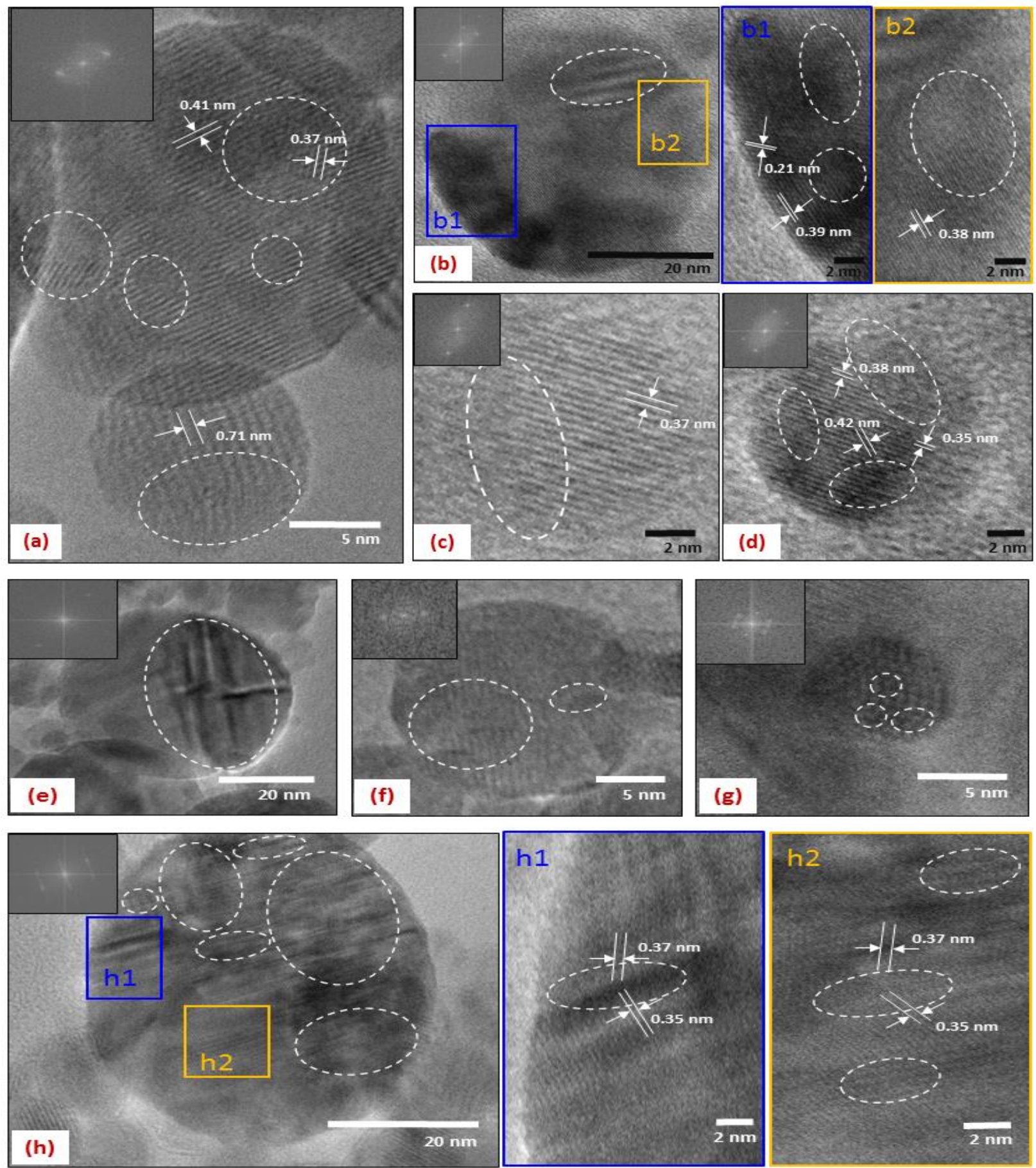

Figure 7.8: HRTEM micrographs of nano TiOx showing single nanospheres (a)-(h) having numerous Oxygen vacancies and defects marked with white dashed circles. The calculated d-spacing is marked and FFT images are given as inset. 
Raman spectra was acquired to further corroborate the XRD and XPS results. A laser beam having a central wavelength of $785 \mathrm{~nm}$ was used for excitation. The measurements taken for both the HDD and LDD-nano $\mathrm{TiO}_{\mathrm{x}}$ synthesised at various laser intensities showed three sharp and distinct peaks, corresponding to the characteristic rutile phase of $\mathrm{TiO}_{2}$, with the peaks of the HDDnano $\mathrm{TiO}_{\mathrm{x}}$ being sharper as seen in Figure 7.9(a)-(f). The symmetricity and the narrowness of the peaks indicated the good crystalline quality of the material [134]. Two of these peaks represent the Raman active modes for rutile: $E_{\mathrm{g}}$ and $A_{1 \mathrm{~g}}$ due to first order Raman scattering [135], [136]. Compared to the standard rutile Raman peaks, these two peaks were red shifted for the synthesised nano $\mathrm{TiO}_{\mathrm{x}}$, indicating structural changes. Such a red shift to lower frequencies is caused due to tensile strain in the crystal lattice, which can be the effect of defects in the crystal [137]. This tensile strain was also confirmed by the large d-spacing values measured in the HR-TEM micrographs.

A comparison study of the shifts shown by HDD-nano $\mathrm{TiO}_{\mathrm{x}}$ and $\mathrm{LDD}$-nano $\mathrm{TiO}_{\mathrm{x}}$ followed. Figure 7.10 shows the shift of the Raman active modes $\left(E_{\mathrm{g}}\right.$ and $\left.A_{1 \mathrm{~g}}\right)$ of both the HDD-nano Titanium Oxide and LDD-nano Titanium Oxide, synthesised at all laser intensities, with respect to the standard Raman active modes of rutile $E_{\mathrm{g}}\left(447 \mathrm{~cm}^{-1}\right)$ and $A_{1 \mathrm{~g}}\left(612 \mathrm{~cm}^{-1}\right)$. Greater shift of LDD- $\mathrm{TiO}_{\mathrm{x}}$ indicated that it had a larger tensile strain. Furthermore, change in particle size has had a proven effect on the Raman spectra, with the Raman bands shifting to a higher wavenumber number as the particle size decreased [138]. As such, a similar observation was expected for the LDD-nano $\mathrm{TiO}_{\mathrm{x}}$, which exhibited lower nanosphere size in the size distribution plots of the HR-TEM data. However, a careful observation of the Raman spectra revealed that it was the peaks corresponding to the Raman active modes $\left(E_{\mathrm{g}}\right.$ and $\left.A_{1 \mathrm{~g}}\right)$ of the HDD-nano $\mathrm{TiO}_{\mathrm{x}}$ that were situated 
at higher frequencies. Compared to $\mathrm{LDD}$-nano $\mathrm{TiO}_{\mathrm{x}}$, the active mode peaks for $\mathrm{HDD}$-nano $\mathrm{TiO}_{\mathrm{x}}$ had shifted by almost $0.5 \mathrm{meV}$ to higher wavenumber, at all at all laser intensities.
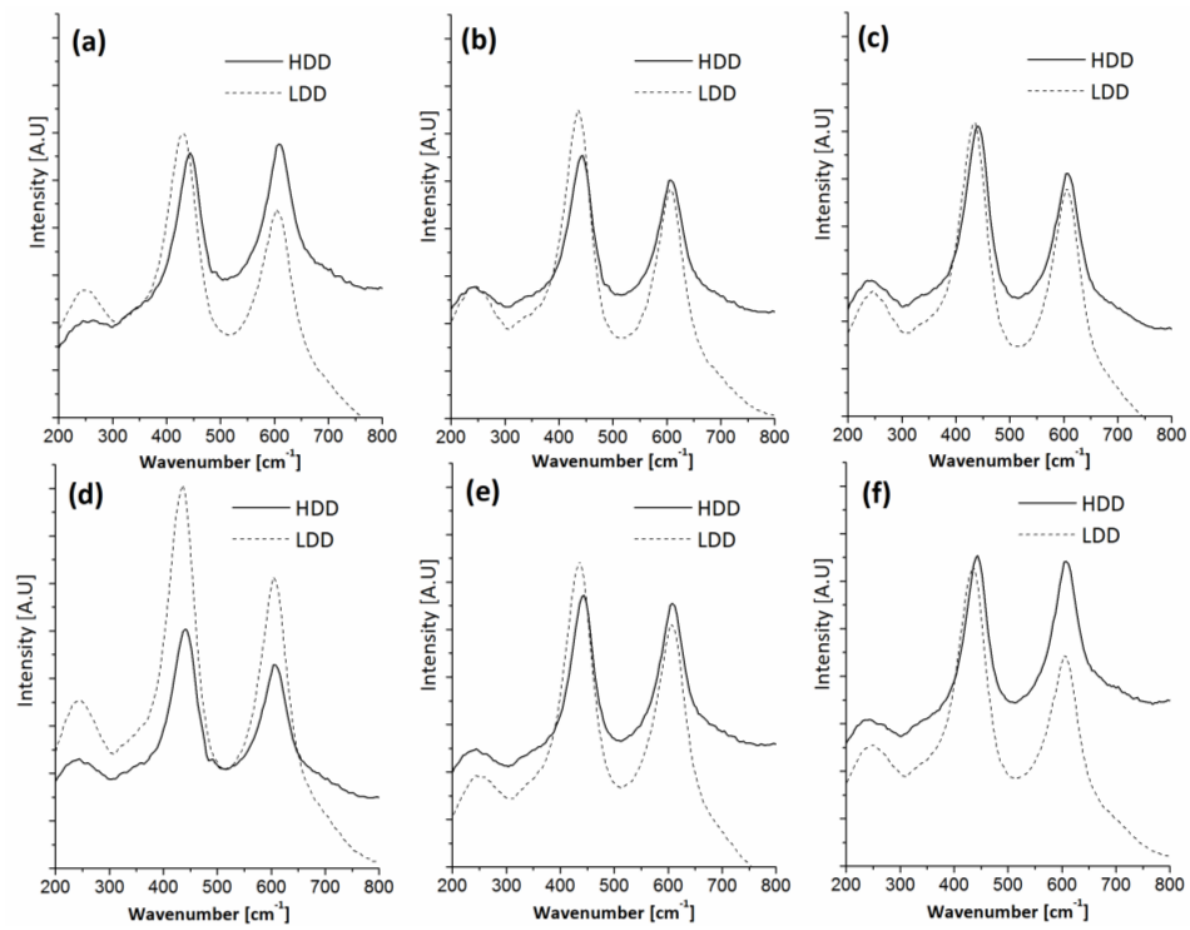

Figure 7.9: Raman spectra of HDD and LDD-nano $\mathrm{TiO}_{\mathrm{x}}$ formed under different laser intensities of (a) $7.4 \times 10^{13} \mathrm{~W} / \mathrm{cm}^{2}$, (b) $1.1 \times 10^{14} \mathrm{~W} / \mathrm{cm}^{2}$, (c) $1.4 \times 10^{14} \mathrm{~W} / \mathrm{cm}^{2}$, (d) $2.2 \times 10^{14} \mathrm{~W} / \mathrm{cm}^{2}$, (e) $4.6 \times 10^{14} \mathrm{~W} / \mathrm{cm}^{2}$, and (f) $7.2 \times 10^{14} \mathrm{~W} / \mathrm{cm}^{2}$

This can be seen in Figure 7.10, where the regions have been encircled. The size and disorders are known to jointly contribute towards the phonon confinement effects [139]. As such, it has been reported earlier that the defects, Oxygen deficiency and greater Oxygen non-stoichiometry in a material influence the Raman spectra by inducing peak shifts and broadening, with modes moving towards higher frequencies [140]-[143]. The sharper peaks blue shift of the Raman modes of the HDD-nano $\mathrm{TiO}_{x}$ with respect to the $\mathrm{LDD}$-nano $\mathrm{TiO}_{\mathrm{x}}$ peaks are further indicative of their welldefined crystal structure along with a greater presence of defects as observed earlier. 


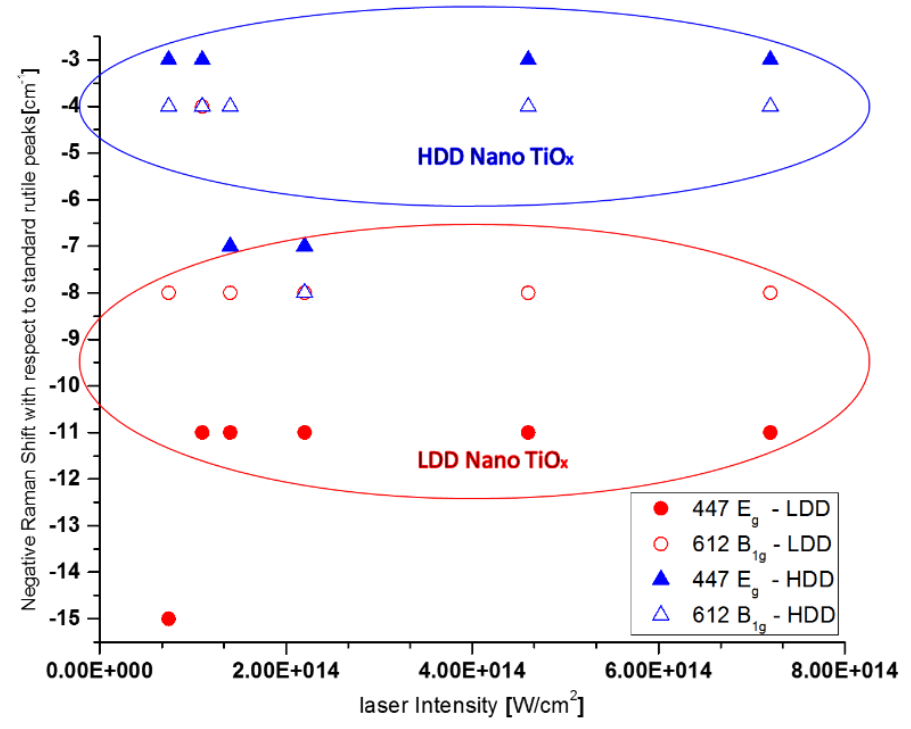

Figure 7.10: Negative Raman shift with respect to the active modes of standard rutile peaks, as observed in the LDD and HDD-nano $\mathrm{TiO}_{\mathrm{x}}$ synthesised at different laser intensities.

The third peak at $244 \mathrm{~cm}^{-1}$, corresponds to the second order Raman scattering arising due to multiphoton scattering process [144], [145]. Peaks corresponding to other Oxide phases of Titanium identified earlier could not be observed in the Raman spectra. This was either due to their Raman inactive nature, as in case of $\mathrm{Ti}_{3} \mathrm{O}$ and $\left(\mathrm{TiO}_{.316}\right)_{7.36}$, or due to insufficient amount of that phase being present for the Raman to detect, as in case of $\mathrm{Ti}_{3} \mathrm{O}_{5}$, which was present in minor traces, as identified earlier by the Rietveld analysis.

\subsection{Absorption Spectrum and Band Gap Calculation}

The absorption properties of the disordered multiphase nano $\mathrm{TiO}_{\mathrm{x}}$ was measured for both the defect poor and high density defect samples, by using a spectrophotometer with a Deuteriumhalogen light source. The absorption spectrum of the base Titanium substrate was also acquired for reference. A broadened absorption spectrum ranging from $300 \mathrm{~nm}$ to $1000 \mathrm{~nm}$ was observed 
for all the synthesised nano $\mathrm{TiO}_{\mathrm{x}}$. The average enhancement when compared with just the base Titanium substrate was almost five times more in the visible region and almost seven times more in the NIR region of the solar spectrum. A steady increase in absorption from UV to deeper visible wavelengths was noticed, which then plateaued beyond $800 \mathrm{~nm}$. It was observed that the HDD-nano $\mathrm{TiO}_{\mathrm{x}}$ exhibited a greater absorption enhancement than the $\mathrm{LDD}$-nano $\mathrm{TiO}_{\mathrm{x}}$ by almost a factor of one in the $300-700 \mathrm{~nm}$ range and by a factor of half in the $800-1000 \mathrm{~nm}$ range, when compared to the base Titanium substrate. This greater enhancement in absorption in the HDD-nano $\mathrm{TiO}_{x}$ was attributed to the presence of more defects and Oxygen vacancies in the self doped nanomaterial [54]. Furthermore, it has been reported earlier that an increase in Oxygen vacancies in Titanium Dioxide results in the generation of more carrier densities in the material, thereby enhancing its electrical conductivity [146]. Also, such defects are known to help in the confinement of phonons as they act like a 'spatially limiting feature' in the grain of the crystal structure [139]. As such, this makes the HDD-nano $\mathrm{TiO}_{\mathrm{x}}$, with its improved opto-electronic properties, a potential candidate for photovoltaic applications. To calculate the bandgap of the nano $\mathrm{TiO}_{\mathrm{x}}$, the Kubelka-Munk (K-M or $\left.F(R)\right)$ method was used, which is based on the equation [147]:

$$
F(R)=\frac{(1-R)^{2}}{2 R}
$$

where $R$ is the reflectance and $F(R)$ is a function based on extinction coefficient $\alpha_{\mathrm{e}}$. The K-M model modified by R. Lopez et. al., and given by the following equation was used to calculate the band gap $E_{\mathrm{g}}[147]$.

$$
\alpha_{\mathrm{e}}(h v) \approx \mathrm{B}\left(h v-E_{\mathrm{g}}\right)^{n}
$$


where $E_{\mathrm{g}}$ is the band gap (eV), $h$ is Planck's constant (J.s), B is absorption constant, $v$ is light frequency $\left(\mathrm{s}^{-1}\right), \alpha_{\mathrm{e}}$ is the extinction coefficient and $n$ is the value for specific transition. The equation was plotted as $\alpha_{e}(h v)^{2}$ verses $E$, which gave the best fit.

The band gap for the LDD-nano $\mathrm{TiO}_{\mathrm{x}}$ was calculated to be around $3.5 \mathrm{eV}$ for all the samples synthesised at different laser intensities. However, the band gap for the HDD-nano $\mathrm{TiO}_{\mathrm{x}}$ was slightly lower and in the range of 3.3-3.4 eV. The absorption spectra of both the HDD and LDD-nano $\mathrm{TiO}_{x}$, synthesised at increasing laser intensity values, have been plotted and shown in Figure 7.11(a)-(f). The band gap plots of only the $\mathrm{HDD}-$ nano $\mathrm{TiO}_{\mathrm{x}}$ have been given as inset, which showed a little variation in value for nano $\mathrm{TiO}_{\mathrm{x}}$ synthesised under different laser intensities. 

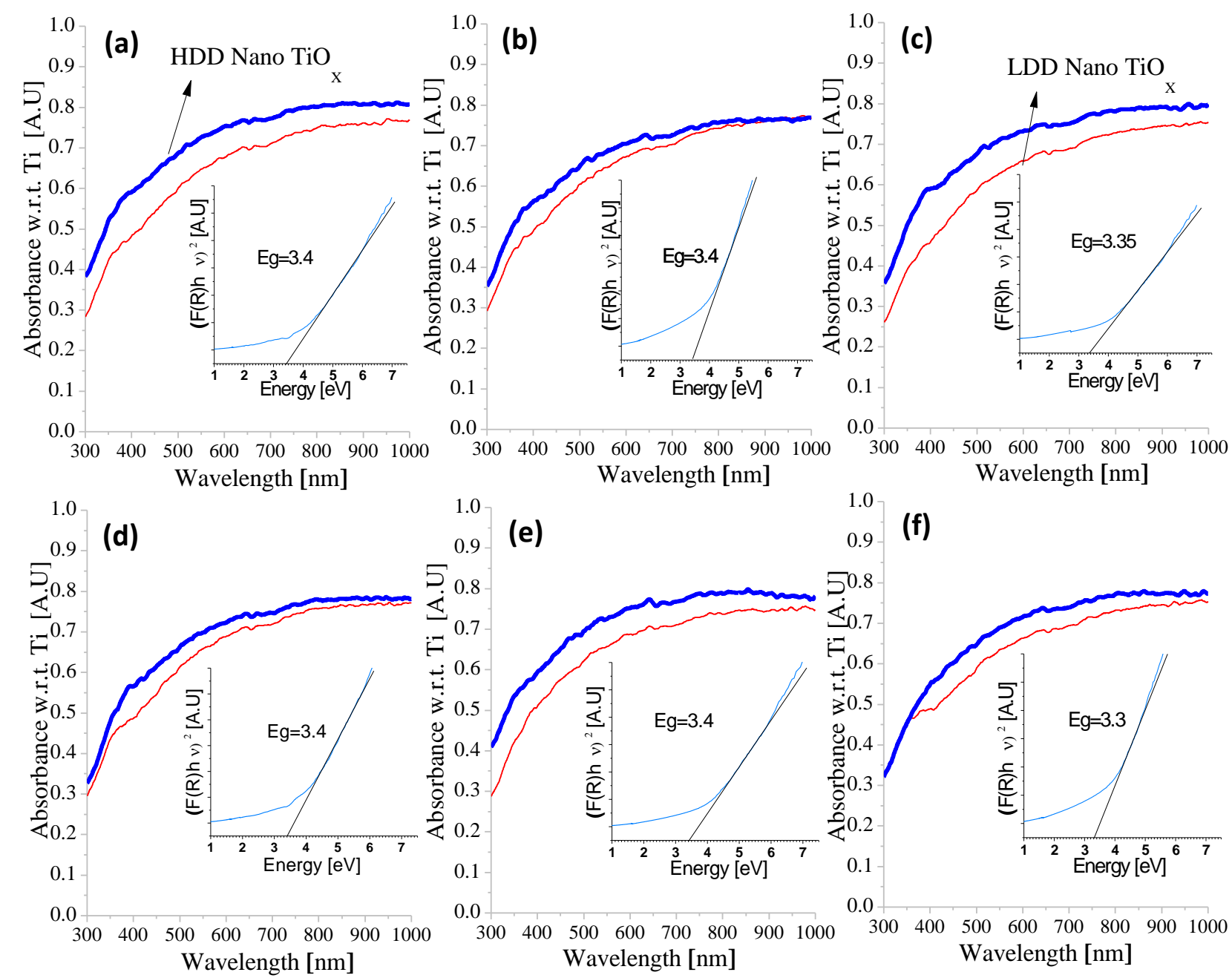

Figure 7.11: Absorption Spectrum of $\mathrm{HDD}^{-n a n o} \mathrm{TiO}_{\mathrm{x}}$ (thick blue line) and $\mathrm{LDD}$-nano $\mathrm{TiO}_{\mathrm{x}}$ (thin red line), synthesised at increasing laser intensities of

(a) $7.4 \times 10^{13} \mathrm{~W} / \mathrm{cm}^{2}$, (b) $1.1 \times 10^{14} \mathrm{~W} / \mathrm{cm}^{2}$, (c) $1.4 \times 10^{14} \mathrm{~W} / \mathrm{cm}^{2}$, (d) $2.2 \times 10^{14} \mathrm{~W} / \mathrm{cm}^{2}$, (e) $4.6 \times 10^{14} \mathrm{~W} / \mathrm{cm}^{2}$, and (f) $7.2 \times 10^{14} \mathrm{~W} / \mathrm{cm}^{2}$. Given inset are the $(F(R) h v)^{2}$ versus $E$ plot, representing the kubelka-munk method to calcualte the corresponding band gap of the HDD-nano $\mathrm{TiO}_{\mathrm{x}}$. 


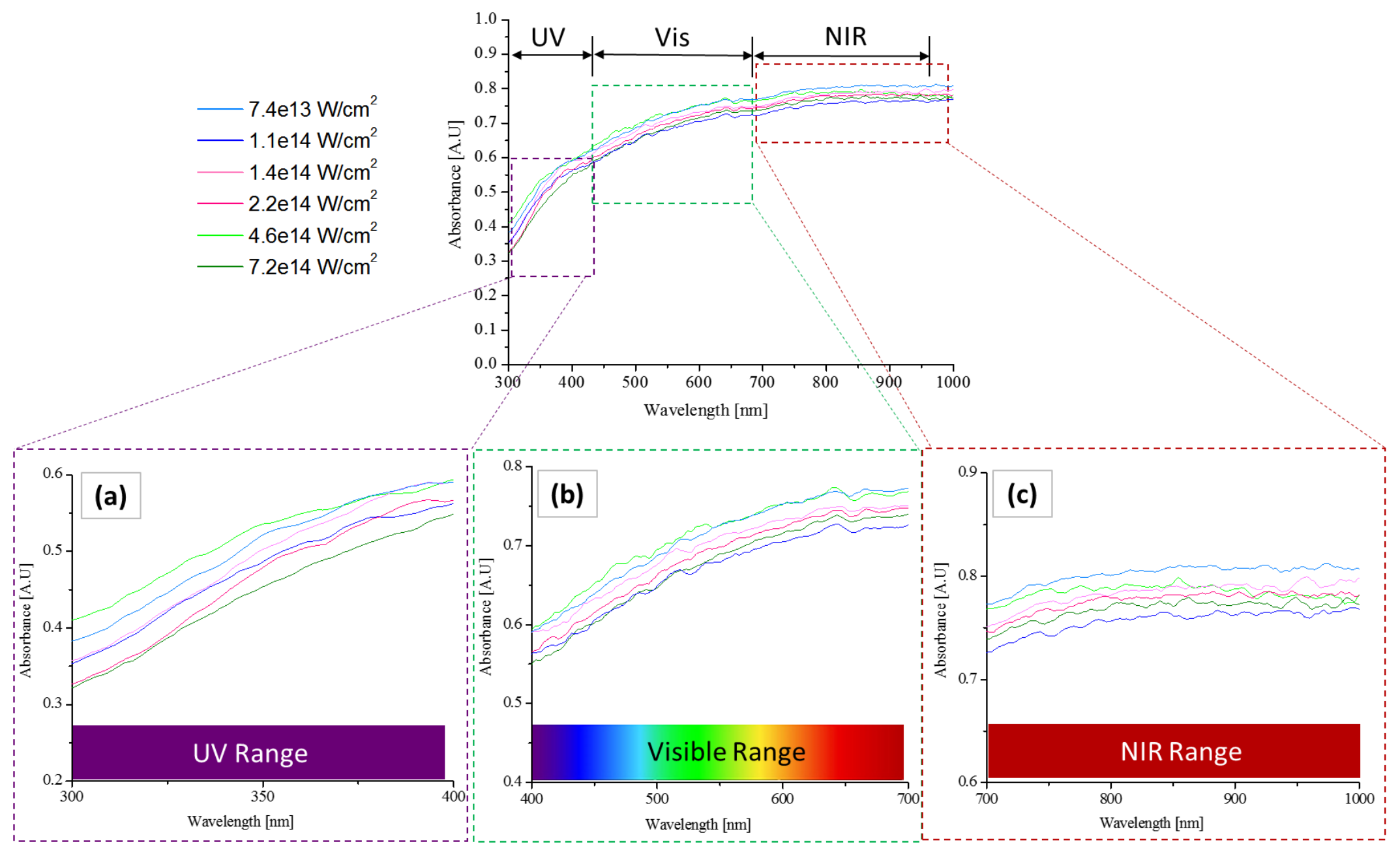

Figure 7.12: Absorption spectrum of the $\mathrm{HDD}$-nano $\mathrm{TiO}_{\mathrm{x}}$ with respect to titanium substrate, when synthesised at increasing laser intensity values. For better representation, the same spectrum is magnified and split into (a) UV range, (b) Visible range, and (c) NIR range. 
To understand the influence of laser intensity at the time of synthesis, on the morphology, phase composition and the subsequent photoabsorptive properties of the $\mathrm{HDD}$-nano $\mathrm{TiO}_{\mathrm{x}}$, its measured absorption spectrum, when synthesised at those laser intensities was plotted together and compared, as seen in Figure 7.12. It was observed that while the difference in absorption was minimal, a little better absorption was favoured by those nanomaterial samples, which were synthesised under slightly lower laser intensities and which corresponded to longer laser pulse widths. The nano $\mathrm{TiO}_{\mathrm{x}}$ synthesised at lower laser intensity values of $4.6 \times 10^{14}$ and $7.4 \times 10^{13} \mathrm{~W} / \mathrm{cm}^{2}$, exhibited the highest absorption enhancement, while the one synthesised at 7.2e14 W/cm ${ }^{2}$ gave the least absorption gain. Figure 7.12(a)-(c), shows the same absorption spectrum, magnified and split into three, corresponding to ultra violet (UV), visible and near infrared range (NIR) absorption. As evident in the plots, the absorption increased rapidly in the UV range. The increase in absorption with wavelength continued into the visible range to a lesser extent and plateaued in the NIR range.

As both emission of photons and Raman scattering are preceded by the optical excitation and absorption of incident photons by the material, to gather more evidence of the photoabsorptive capability of the HDD-nano $\mathrm{TiO}_{\mathrm{x}}$, a spectroflurometer and a Dual wavelength Raman were used to acquire its excitation/emission spectra and Raman spectra respectively [97]. For the spectroflurometer study, the nano $\mathrm{TiO}_{\mathrm{x}}$ was detached and dispersed homogenously in a nonreactive liquid medium at room temperature (Figure 7.13(a) and (b)). As seen in Figure 7.13(b), the HDD-nano $\mathrm{TiO}_{\mathrm{x}}$, synthesised at a lower laser intensity value, which exhibited the highest absorption also showed the higher emission intensity, which suggested greater photon excitation and absorption, so desired for potential photovoltaic applications. Further study of the spectra lead to a critical observation. Longer excitation wavelengths in the deeper visible and NIR range of 
$600-900 \mathrm{~nm}$ lead to emission spectra in the wavelength range of $350-400 \mathrm{~nm}$. This suggested an interesting up-conversion behaviour being shown by the HDD-nano TiOx which shall be further looked into, in our future studies. Photon upconversion is said to occur when two or more photons of lower energy are absorbed and then emitted as a single photon of higher energy. Additionally, there was minimal emission at longer wavelengths indicating that minimum energy was lost to heating. This longer wavelength emission, which leads to the phenomenon of fluorescence was seen only for excitation wavelengths ranging from $250-400 \mathrm{~nm}$, which corresponded to a dip in absorption for the same spectrum range. This study also indicated the fact that these excited electrons emitted by the nanospheres dispersed in the solution for the study, were successfully trapped as a result of the self-assembled three-dimensional structural network of nano $\mathrm{TiO}_{\mathrm{x}}$ which helped achieve a broadened and enhanced absorption spectrum. Furthermore, emission was also indicative of the fact that these excited electrons were not lost to recombination, which is a critical aspect for potential photo absorber materials. As expected, a much lower intensity of emission was seen for the nano $\mathrm{TiO}_{\mathrm{x}}$ synthesised at $7.2 \times 10^{14} \mathrm{~W} / \mathrm{cm}^{2}$ (Figure $7.13(\mathrm{~b})$ ). Next, the dual wavelength Raman study was done at two laser beam wavelengths of $532 \mathrm{~nm}$ and $785 \mathrm{~nm}$, for the HDD-nano $\mathrm{TiO}_{\mathrm{x}}$ synthesised at all laser intensities, to obtain more evidence of their photon absorption capability. As expected, significantly greater amount of Raman enhancement was seen at $785 \mathrm{~nm}$ than at $532 \mathrm{~nm}$, indicating more absorption at the longer wavelength as evidenced by the absorption plots in Figure 7.12. Figure 7.13 shows the excitation emission spectra and the dual wavelength Raman spectra for the $\mathrm{HDD}$-nano $\mathrm{TiO}_{\mathrm{x}}$ synthesised at laser intensities of $7.2 \times 10^{14} \mathrm{~W} / \mathrm{cm}^{2}$ and $4.6 \times 10^{14} \mathrm{~W} / \mathrm{cm}^{2}$. The excitation emission spectra for LDD-nano $\mathrm{TiO}_{\mathrm{x}}$ synthesised at laser intensities of $7.2 \times 10^{14} \mathrm{~W} / \mathrm{cm}^{2}$ and $4.6 \times 10^{14} \mathrm{~W} / \mathrm{cm}^{2}$ has also been given in Figure 7.14, which exhibited, comparatively less excitation behaviour than LDD-nano $\mathrm{TiO}_{\mathrm{x}}$. As a 
final part of our study, we performed additional experiments at the same laser intensities and poor Oxygen conditions. However, the flow rate of the Nitrogen gas was now increased to 6 SCFH and the absorption spectrum of the nano $\mathrm{TiO}_{\mathrm{x}}$ thus synthesised was measured. At all conditions, no further gain in absorption was achieved. In fact a slight reduction in absorption was observed. This indicates that a saturation state was achieved by the excessive ionic content in the laser plasma plume, leading to reduced ion mobility due to the pressure exerted by the dense ion gas cloud on to the laser plume and to the generation of higher Oxide formation. As such, no further improvement in the crystal growth and subsequent optical absorption properties of the HDD-nano $\mathrm{TiO}_{\mathrm{x}}$ was observed. 

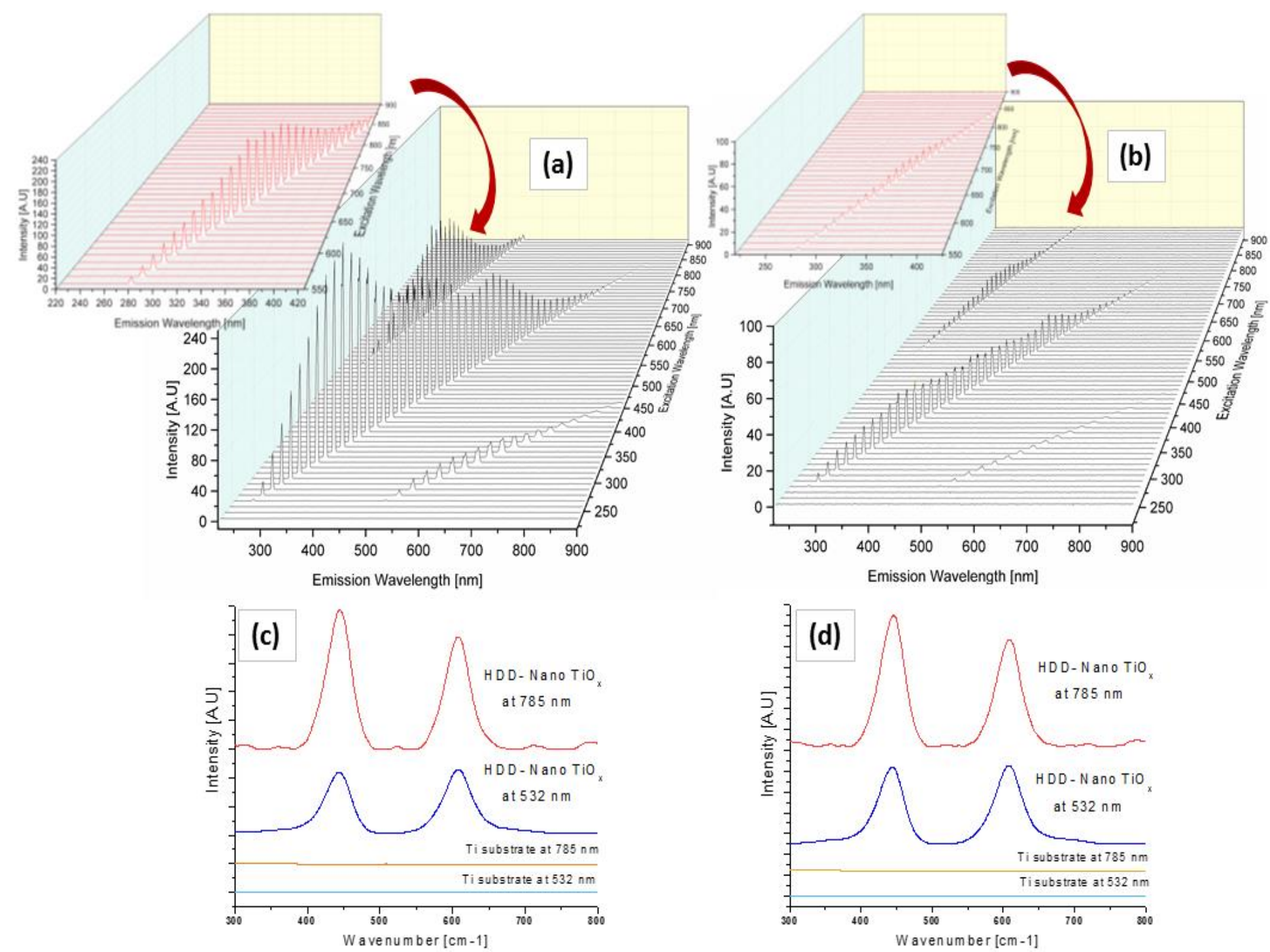

Figure 7.13: The excitation/emission spectra of $\mathrm{HDD}$-nano $\mathrm{TiO}_{\mathrm{x}}$ at laser intensity of (a) $4.6 \times 10^{14} \mathrm{~W} / \mathrm{cm}^{2}$ and (b) $7.2 \times 10^{14} \mathrm{~W} / \mathrm{cm}^{2}$, and the corresponding dual Raman spectra is given in (c) and (d). The area of the spectra indicating possible up-conversion is magnified and given as inset. 

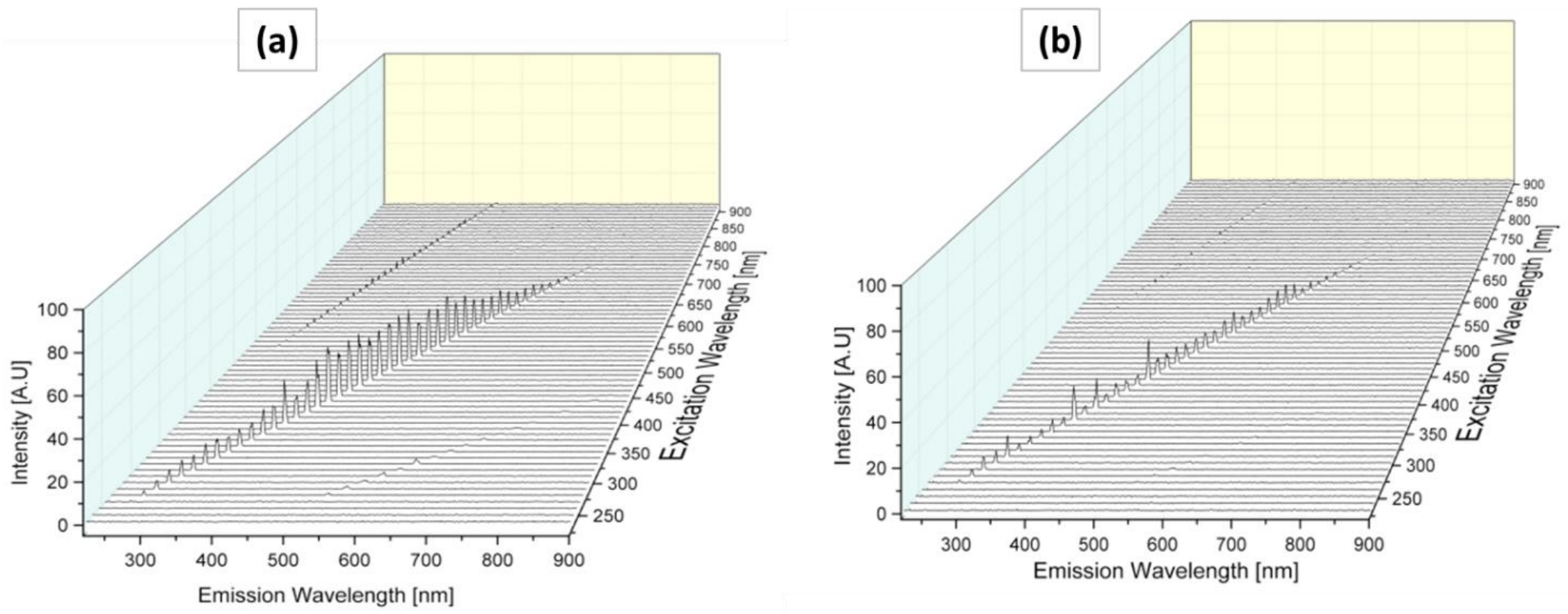

Figure 7.14: The excitation/emission spectra of LDD-nano $\mathrm{TiO}_{\mathrm{x}}$ at laser intensity of (a) $4.6 \times 10^{14} \mathrm{~W} / \mathrm{cm}^{2}$ and (b) $7.2 \times 10^{14} \mathrm{~W} / \mathrm{cm}^{2}$. 


\subsection{Summary}

In this chapter, we have proposed a disordered multiphase nano $\mathrm{TiO}_{\mathrm{x}}$, rich in defects and Oxygen vacancies. Defects were induced in the multiphase nano $\mathrm{TiO}_{\mathrm{x}}$ to varying degrees by altering the surrounding ionising medium during its synthesis. A laser plasma plume, consisting of ionic and atomic species, generated due to laser material interaction, governs the vapour condensation mechanism and the subsequent self-assembly of nano network. Its ionic content was altered by introducing Oxygen poor and Oxygen rich conditions in the surrounding medium. This activated and influenced the physio-chemical properties of the multiphase nano $\mathrm{TiO}_{\mathrm{x}}$, by causing a shift in

its stoichiometry to lower Oxides of Titanium and creating numerous Oxygen vacancies and defects to varying degrees. A disordered structure, rich (HDD-nano $\mathrm{TiO}_{\mathrm{x}}$ ) and poor $\left(\mathrm{LDD}-\right.$ nano $\mathrm{TiO}_{\mathrm{x}}$ ) was achieved. The optical properties of this disordered multiphase nano $\mathrm{TiO}_{\mathrm{x}}$ was investigated and its viability in photon harvesting was determined. A five-fold increment in absorption in the visible region and a seven-fold increment in the near-infrared region was achieved, compared to the base Titanium substrate. 


\section{Chapter 8}

\section{Proposed Applications for Multiphase}

\section{Nano TiOx}

This chapter briefly touches upon possible uses of the novel multiphase nano $\mathrm{TiO}_{\mathrm{x}}$ in different applications. Photon harvesting applications like photovoltaics and photocatalysis could be areas of implementation for such a nanomaterial due to the broadened absorption range, as discussed in earlier chapters. Apart from these two applications, its use could also be extended to the detection

of analytes for chemical sensing applications, by virtue of its appreciable Raman enhancement capability. Furthermore, a surface wettability test conducted on the material exhibited a hydrophilic type of behaviour, which could help extend its feasibility in wider applications like self-cleaning and anti-fogging surfaces.

As mentioned earlier in Chapter $1, \mathrm{TiO}_{2}$ is the most common oxide of Titanium which is widely used as a photo catalyst in numerous applications. As such, it seems appropriate to compare the absorption spectrum of the unique self-assembled multiphase nano $\mathrm{TiO}_{\mathrm{x}}$ network with the absorption spectrum of $\mathrm{TiO}_{2}$. As seen in previous chapters, the absorption spectra of multiphase nano $\mathrm{TiO}_{\mathrm{x}}$ were acquired by taking the base Titanium substrate as the reference, on which they had been synthesised at different laser processing conditions. The various samples of multiphase nano $\mathrm{TiO}_{\mathrm{x}}$ had been then compared relative to each other. However, to ensure that a valid 
comparison with $\mathrm{TiO}_{2}$ could be made, the absorption spectrum was then taken by utilizing a standard white reference (Avantes WS-2 tile).

Figure 8.1 shows this comparison of the absorption spectrum of $\mathrm{TiO}_{2}$ with that of $\mathrm{HDD}$-nano $\mathrm{TiO}_{\mathrm{x}}$ and LDD-nano $\mathrm{TiO}_{x}$. As seen, both HDD- and LDD-nano $\mathrm{TiO}_{\mathrm{x}}$ demonstrated absorption covering a wider wavelength range of the spectrum, as compared to $\mathrm{TiO}_{2}$. The solar irradiance spectrum has also been given in the background to indicate the total energy of the incident photons that can be exploited for harvesting. $\mathrm{TiO}_{2}$ is capable of capturing only UV photons (which accounts for approximately $7 \%$ of total solar irradiance energy). In contrast, nano $\mathrm{TiO}_{\mathrm{x}}$ shows a broadband absorption spectrum in the wavelength of 300 to $1000 \mathrm{~nm}$, which accounts for a much larger portion of the incident solar irradiance energy. This makes it a potential candidate for photon harvesting applications.

An additional observation was made about the colour of these self assembled nano $\mathrm{TiO}_{\mathrm{x}}$ networks. Samples were greyish, but the LDD-nano $\mathrm{TiO}_{\mathrm{x}}$ samples were more blue in colour. A look at the absorption spectrum in Figure 8.1 further corroborates this observation. A slight dip was seen in the absorption spectrum of LDD-nano $\mathrm{TiO}_{\mathrm{x}}$ in the $400-500 \mathrm{~nm}$ range, which corresponds to the blue wavelengths of the visible spectrum. On the contrary, $\mathrm{HDD}$-nano $\mathrm{TiO}_{\mathrm{x}}$ was shown to have a steady absorption spectrum throughout, which explains why it was more grey in colour. 


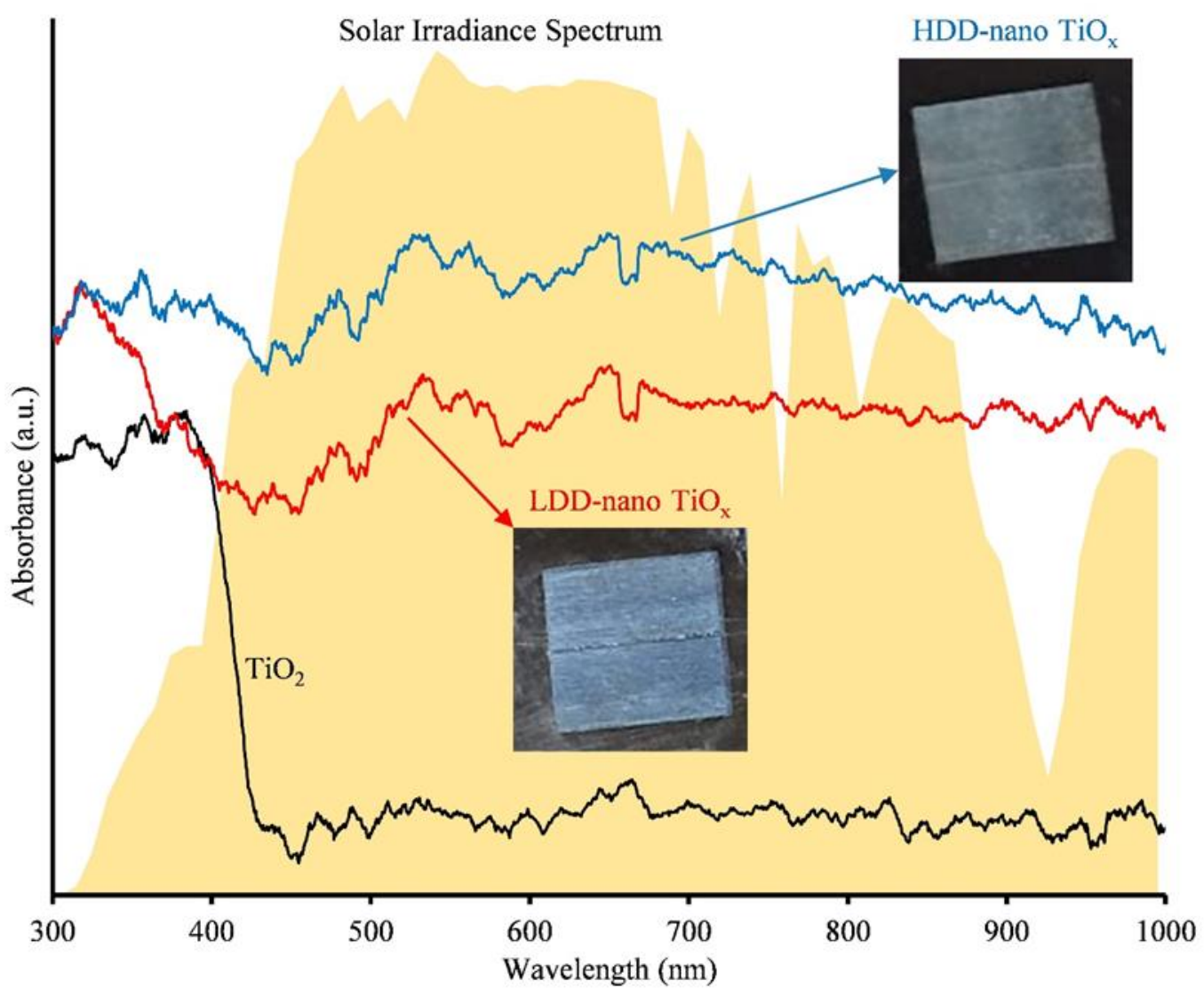

Figure 8.1 Comparison of the absorption spectrum of the $\mathrm{TiO}_{2}$ with that of $\mathrm{HDD}-\mathrm{Nano}^{\mathrm{TiO}} \mathrm{x}$ and LDD-Nano $\mathrm{TiO}_{\mathrm{x}}$.

The more bluish colour of the LDD-Nano $\mathrm{TiO}_{\mathrm{x}}$ when compared with the grey coloured $\mathrm{HDD}-\mathrm{Nano} \mathrm{TiO}_{\mathrm{x}}$ is clearly seen in the photographs provided. The solar irradiance spectrum [148] is shown in back drop for comparison.

Moving forward, another potential application for nano $\mathrm{TiO}_{\mathrm{x}}$ is related to the the Raman spectral studies described in Chapter 7, where peaks of reasonable intensity were observed for HDD-nano $\mathrm{TiO}_{\mathrm{x}}$. Furthermore, a high amount of photon excitation was also seen in the excitation/emission spectra. By virtue of increased absorption of incident light, it is well known that Raman enhancement is caused due to a highly amplified electromagnetic field, which usually develops on a nanostructured material surface. Furthermore, Surface Enhanced Raman Scattering (SERS) phenomenon has not only been attributed to a amplified electromagnetic field at the material surface, but also due to a chemical enhancement caused by charge transfer resonance 
[149]-[151]. This propelled an attempt to quantify the latent Raman sensing capability of HDD-nano $\mathrm{TiO}_{\mathrm{x}}$. A major use of materials showing SERS behaviour is towards the detection of analytes for chemical sensing applications. As such, Crystal Violet (CV), a chemical dye was used to test the ability of the synthesised nanomaterial to sense the presence of such a chemical analyte. $\mathrm{CV}$ dye has a large Raman cross section and is thus commonly used to demonstrate SERS. As seen earlier, $\mathrm{HDD}$-nano $\mathrm{TiO}_{\mathrm{x}}$ had exhibited higher photon absorption (Figure 7.12), and more intense Raman peaks had been observed around $785 \mathrm{~nm}$ (Figure 7.13); hence the Raman excitation wavelength was chosen to be this value. To activate SERS on the HDD-nano $\mathrm{TiO}_{\mathrm{x}}, \mathrm{CV}$ dye was coated onto its surface (see Figure 8.2), and the Raman spectra was acquired. The enhancement factor $(\mathrm{EF})$ was then calculated for the $\mathrm{HDD}$-nano $\mathrm{TiO}_{\mathrm{x}}$, which is a value used to evaluate the Raman sensing capability of a material.

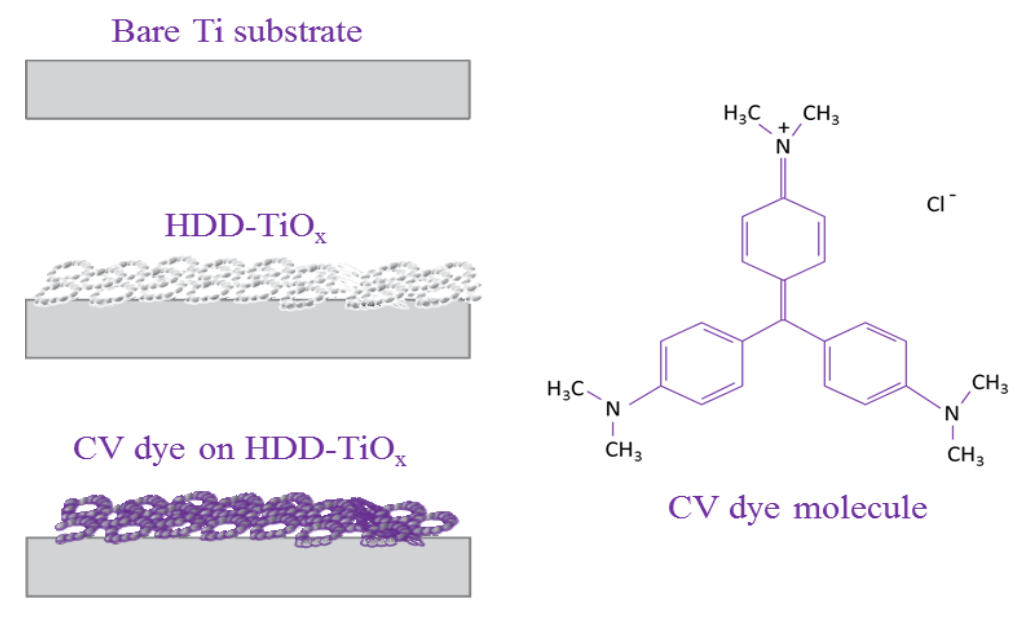

Figure 8.2: Graphical illustration of coating HDD-nano TiOx with CV dye. 

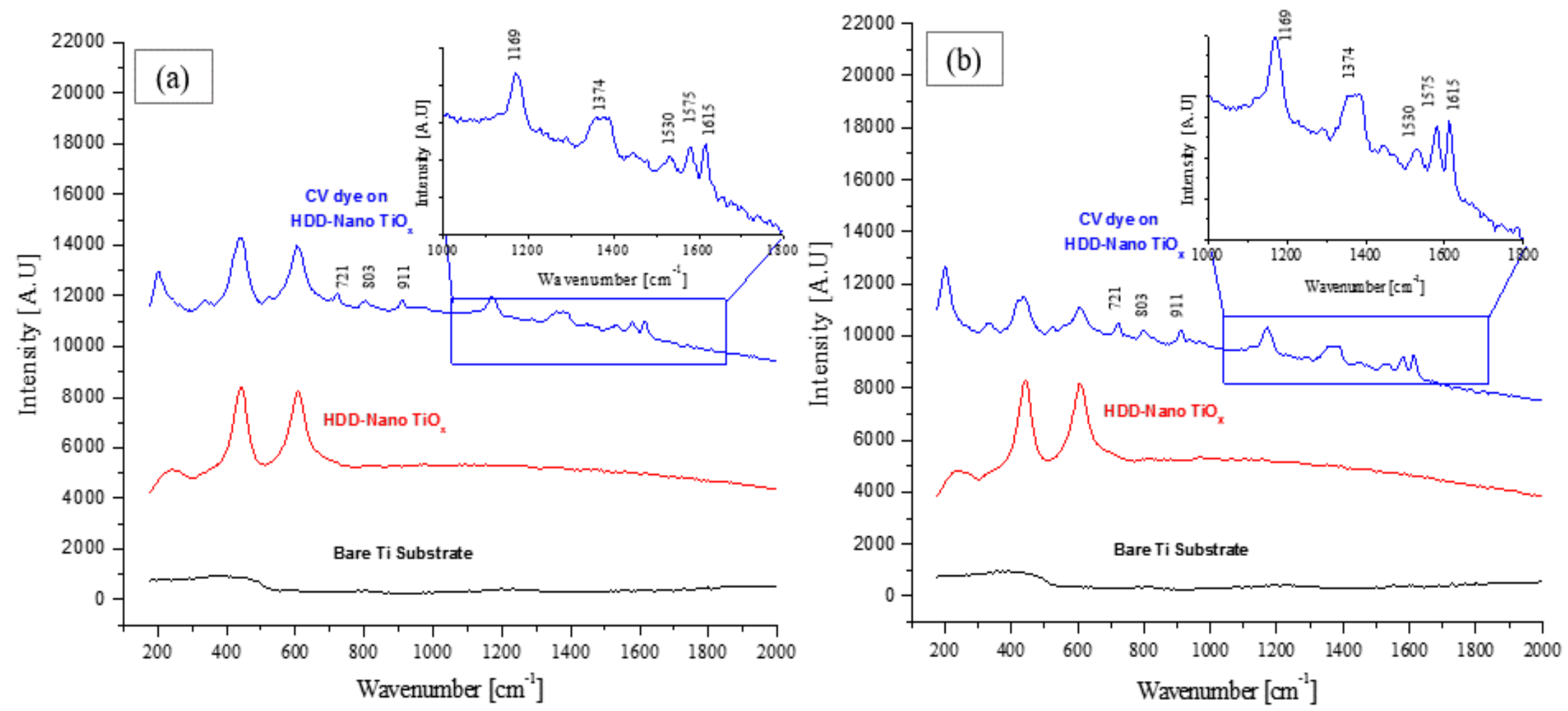

Figure 8.3: Raman spectra CV dye on HDD-nano TiOx synthesised at laser intensities of (a) $4.6 \mathrm{e} 14 \mathrm{~W} / \mathrm{cm}^{2}$ and $7.2 \mathrm{e} 14 \mathrm{~W} / \mathrm{cm}^{2}$. The Raman spectra of just the HDD nano TiOx and only the bare Ti substrate has also been plotted for comparison. The characteristic peaks of the CV dye have been marked. Magnified plots have been given as inset for the Raman spectra of CV dye on $\mathrm{HDD}$-nano $\mathrm{TiO}_{\mathrm{x}}$ for clarity of peaks. 
Figure 8.3(a) and (b) shows the Raman spectra of the CV dye on HDD-nano $\mathrm{TiO}_{\mathrm{x}}$ when synthesised at laser intensities of $4.6 \mathrm{e} 14 \mathrm{~W} / \mathrm{cm}^{2}$ and $7.2 \mathrm{e} 14 \mathrm{~W} / \mathrm{cm}^{2}$ respectively. The Raman spectra of just the HDD-nano $\mathrm{TiO}_{\mathrm{x}}$ and only the bare $\mathrm{Ti}$ substrate has also been plotted for comparison. The procedure and calculation of the enhancement factor values was adopted by using the equations given for similar morphology nanostructures by Cong et al. in [151] and has been given in appendix $\mathrm{B}$. The most prominent $\mathrm{CV}$ dye peak in the Raman spectra was seen at a wavenumber of $1169 \mathrm{~cm}^{-1}$ and this peak was used to calculate the enhancement factor. An enhancement factor of $2.5 \times 10^{7}$ and $3.2 \times 10^{7}$ was determined for the HDD-nano $\mathrm{TiO}_{\mathrm{x}}$ synthesised at laser intensities of $4.6 e 14 \mathrm{~W} / \mathrm{cm}^{2}$ and $7.2 e 14 \mathrm{~W} / \mathrm{cm}^{2}$ respectively. Such an enhancement factor of the order of magnitude $10^{7}$ calculated for HDD-nano $\mathrm{TiO}_{\mathrm{x}}$ is appreciably high, considering that most plasmon free $\mathrm{TiO}_{2}$ based semiconductor nanomaterials have observed an enhancement factor of $10^{4}$ [150]. Presence of vacancies and defects have recently been observed to cause a high SERS sensitivity in semi-conductors [151]. This could explain the high orders of magnitude of the enhancement factor calculated for the HDD-nano $\mathrm{TiO}_{\mathrm{x}}$. Further investigation into the tuning of the properties of $\mathrm{HDD}$-nano $\mathrm{TiO}_{\mathrm{x}}$ for better $\mathrm{CV}$ dye enhancement is necessary. The characteristic SERS nature exhibited by this semi-conducting HDD-nano $\mathrm{TiO}_{\mathrm{x}}$ nanomaterial, which resulted in an EF value of the order of $10^{7}$ for $\mathrm{CV}$ dye, can also widen its applicability in the field of chemical sensing.

Another potential area of application of nano $\mathrm{TiO}_{\mathrm{x}}$ can be in self-cleaning and anti-fogging materials. $\mathrm{TiO}_{2}$ is widely considered for such applications by the virtue of its hydrophilic properties under UV irradiation [152]. This high wettability of $\mathrm{TiO}_{2}$ is known to be a function of its photosensitivity and geometric structure [153], [154]. Due to similar characteristics, it is hypothesized that HDD-nanoTiOx could have similar hydrophilicity. Contact Angle is typically 
used as a measure to determine the surface wettability of a material. This value is defined as the angle between a tangent drawn from the edge of the liquid droplet to the solid surface of the material. A rudimentary set up was arranged to measure changes in the contact angle upon UV and visible light irradiation. Two samples of $\mathrm{HDD}$-nano $\mathrm{TiO}_{\mathrm{x}}$ were prepared under similar laser processing conditions $\left(4.6 e 14 \mathrm{~W} / \mathrm{cm}^{2}\right.$ laser intensity) and a $2.5 \mu 1$ quinine water droplet was deposited onto to the surface of the samples. For UV irradiation, a UV lamp (365 $\mathrm{nm}$ radiation at $100 \mathrm{~W})$ and for visible light irradiation, a halogen lamp (21 V/150 W) was used. (These rated powers are the electrical rating of the lamps.) The samples were kept at a distance of $2 \mathrm{~cm}$ from the lamp source. The contact angle was measured immediately upon irradiation and thereafter at several time intervals. It is to be noted that the incident light intensity could not be directly controlled, due to limitations of available equipment, and hence a ceteris paribus comparison wouldn't be appropriate between the UV and visible light irradiation experiments. If desired, a more controlled experiment (as conducted in [155] and [156]) could be carried out to allow a direct comparison, which has been deemed beyond the scope of this dissertation.

As seen in Figure 8.4(a) and (b), the contact angle decreases while irradiated with both UV and visible lights, and tended towards hydrophilicity ( $<20$ degrees) after about 300 seconds of exposure time. Hydrophilic behaviour was observed for $\mathrm{HDD}$-nano $\mathrm{TiO}_{\mathrm{x}}$ under both visible and UV irradiation. This behaviour is similar to the other photoinduced type of hydrophilic behaviour. For $\mathrm{TiO}_{2}$, it has been attributed to structural surface changes brought about by photon generated charge carriers [153], [157], which create $\mathrm{Ti}^{3}+$ states and oxygen vacancies on the surface, causing water to be dissociated and adsorbed. Hydrophilic $\mathrm{OH}$ groups are then formed on the surface which is responsible for the surface wetting. Similarly, in $\mathrm{HDD}$-nano $\mathrm{TiO}_{\mathrm{x}}$ the presence of high density of oxygen defects could be a reason for it exhibiting a hydrophilic type of behaviour. 

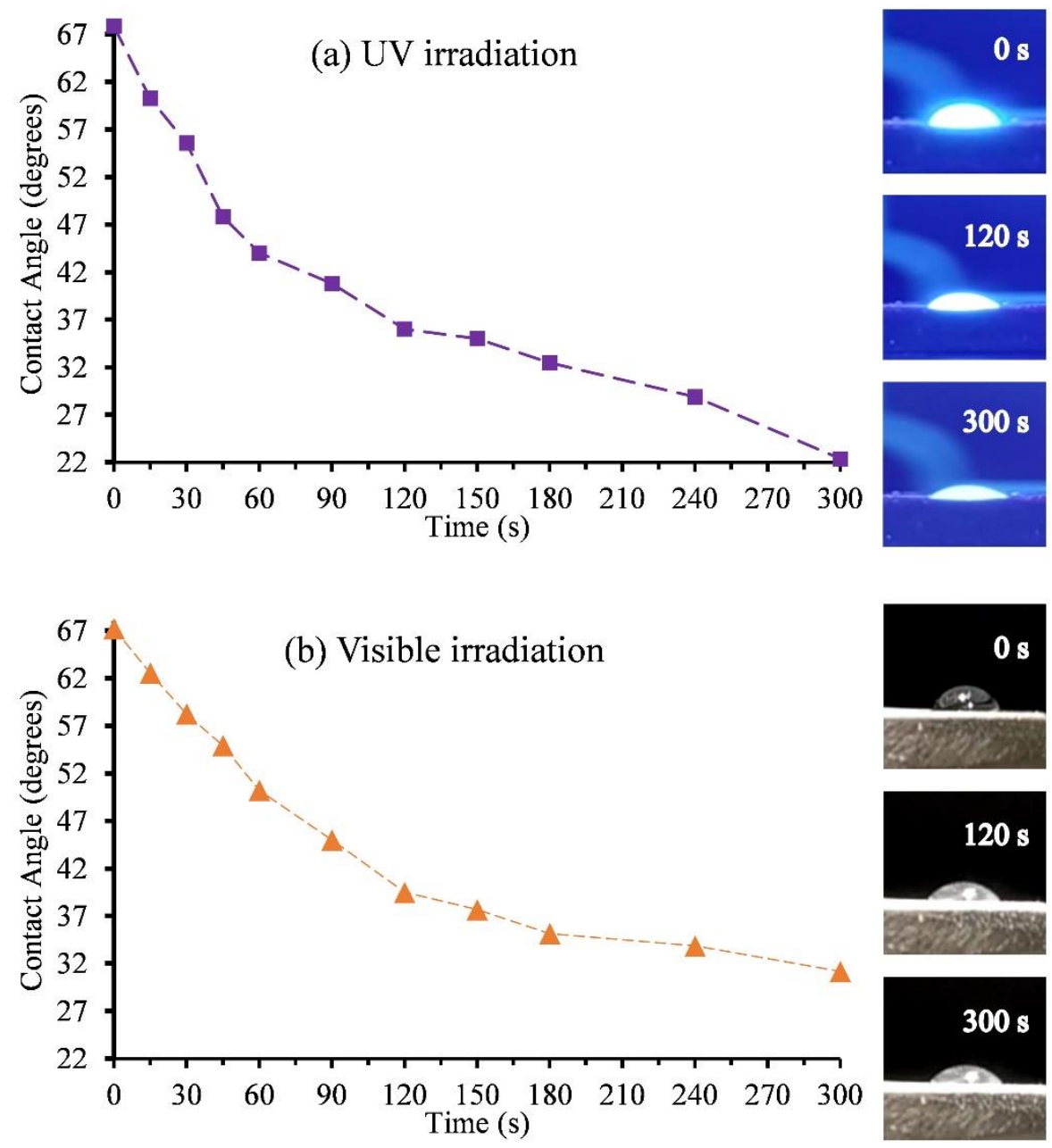

Figure 8.4: Variation of the contact angle of the liquid droplet deposited on HDD-nano $\mathrm{TiO}_{\mathrm{x}}$ when subjected to (a) UV irradiation (b) visible irradiation. Photographs on the right show the shape of the droplet on $\mathrm{HDD}^{-n a n o} \mathrm{TiO}_{\mathrm{x}}$ at different exposure times during irradiation.

The study shown in Figure 8.4 is not intended to be exhaustive, but instead as a rudimentary test which indicates nano $\mathrm{TiO}_{\mathrm{x}}$ to be exhibit some hydrophilic properties. Further experimental studies need to be conducted to do an in-depth surface wettability property analysis of the multiphase nano $\mathrm{TiO}_{\mathrm{x}}$ material developed in this thesis. As described in [157], the photo induced wettability of materials can be characterised by doing comparison studies on structural changes, hardness, irradiation intensity and dark storage environment experiments. Future work based on these types 
of studies could help widen the application range of multiphase nano $\mathrm{TiO}_{\mathrm{x}}$ to self-cleaning and anti-fogging materials.

This chapter thus provides a brief example of the potential application of the multiphase nano $\mathrm{TiO}_{\mathrm{x}}$ which extends beyond energy harvesting. Similar application focussed studies on the multiphase nano $\mathrm{TiO}_{\mathrm{x}}$ can be pursued, which could further demonstrate the feasibility of its enhanced and broadened optical absorption properties. 


\section{Chapter 9}

\section{Summary and Future Research}

\subsection{Summary}

In this dissertation, a novel multiphase Oxide of Titanium in the form of a 3D nanonetwork was synthesised via ultrashort laser material interaction. Its photon absorption capability was demonstrated and improved upon for potential photon harvesting applications. Numerous concepts like phase transformation, surface and material characterisation, physics of ultrashort laser material interaction and nanostructure formation, target surface temperature analysis, plasmon resonance and structure disordering were elucidated.

The main objective of this dissertation was to develop a nanomaterial based on titanium oxides, which could attain measurable absorption over a broadened range of the absorption spectrum, that included both the visible and NIR region.

Transformative phase functionalization of Titanium was achieved by its unique interaction with ultra-short laser pulses of femtosecond duration. Characterisation studies identified it to be composed of multiple Titanium Oxide phases: $\mathrm{Ti}_{3} \mathrm{O},\left(\mathrm{TiO}_{.716}\right)_{3.76}$ and $\mathrm{TiO}_{2}$ (rutile). A remarkable gain in the absorption of photons in the vis-NIR region $(300-1000 \mathrm{~nm})$ was shown by this functionalised Titanium, whose band gap averaged around $2.39 \mathrm{eV}$. With respect to base Titanium, 
a three-fold absorption gain in the visible region and a four-fold absorption gain in the NIR region of the solar spectrum was achieved.

The multiphase Titanium Oxide material achieved via phase functionalization of Titanium exhibited a broadened and enhanced absorption. Fabrication of a complex 3D nano network composed of this multiphase $\mathrm{TiO}_{\mathrm{x}}$ was achieved by modifying the nature of the ultrashort laser pulses and their subsequent interaction with the Titanium material. A three-fold enhancement in the deeper visible region of the spectrum and a six-fold enhancement in the NIR region, when compared to the absorption spectrum of the Titanium substrate, was exhibited by the multiphase nano $\mathrm{TiO}_{\mathrm{x}}$ network. A study of variation in the phase composition and morphology/size of the nanospheres constituting the nano network, with change in the laser processing conditions was conducted. The subsequent effect on its photon absorptive capability was then studied.

Plasmon resonance was then successfully utilized by hybridising the multiphase nano $\mathrm{TiO}_{\mathrm{x}}$ network. The plasmonic resonance behaviour of $\mathrm{Au}$ and $\mathrm{Au} / \mathrm{Pd}$ alloy metal plasmons was exploited to acquire such a hybridised multiphase nano $\mathrm{TiO}_{\mathrm{x}}$ network exhibiting almost a six-fold absorption enhancement in the deeper visible region. Studies examined the impact of various factors like the size, composition and degree of hybridisation of the self-assembled 3D multiphase nano Titanium Oxide network on its photon absorption capability.

A disordered multiphase nano $\mathrm{TiO}_{\mathrm{x}}$ with varying density of defects was then synthesised and their contribution to broadened absorption was exhibited. $\mathrm{HDD}$-nano $\mathrm{TiO}_{\mathrm{x}}$ and $\mathrm{LDD}$-nano $\mathrm{TiO}_{\mathrm{x}}$ were obtained by introducing poor and rich Oxygen conditions in the surrounding medium during their synthesis. By synthesising HDD-nano $\mathrm{TiO}_{\mathrm{x}}$ a multiphase nanomaterial rich in defects and Oxygen 
vacancies, compared to the base titanium substrate, a five-fold increment in absorption in the visible region and a seven-fold increment in the near-infrared region was accomplished.

The main contributions of this dissertation are:

- Achieved enhanced and broadened absorption by developing a 'self-doped' HDD-nano $\mathrm{TiO}_{\mathrm{x}}$ network which is rich in defects and vacancies.

- Developed the photoabsorptive multiphase nano $\mathrm{TiO}_{\mathrm{x}}$ network, which is $3 \mathrm{D}$ and free of dopants, and capable of absorbing photons in the broad-spectrum range of 300-1000 nm. Further optimisation of its absorption was achieved by its Plasmon hybridisation.

- Demonstrated phase functionalization of Titanium to attain enhanced absorption in vis-NIR region.

\subsection{Recommended Future Research}

While a unique material has been synthesised and investigated to enhance and broaden its absorption spectrum, many exciting avenues still exist to continue to explore the study of multiphase nano $\mathrm{TiO}_{\mathrm{x}}$. These range from further structural and phase modification of the nanomaterial via different mechanisms to their potential implementation in photon harvesting devices. The following work is proposed to apply the insight gathered in this dissertation, towards furthering and expanding the objective of achieving a perfect photon absorber.

- To functionalise Titanium and generate a nano $\mathrm{TiO}_{\mathrm{x}}$ network with ultrashort laser pulses at different wavelengths. The temporal effective delivery of energy to the target material changes with the wavelength of a laser pulse. This can have considerable effects on the 
morphology and phase transformation process of nanomaterial synthesis and its subsequent absorption properties.

- To regulate and tune the number of oxygen vacancies in the $\mathrm{HDD}$-nano $\mathrm{TiO}_{\mathrm{x}}$ by using a vacuum chamber during laser synthesis.

- Further increase the photon absorptive capability of multiphase nano $\mathrm{TiO}_{\mathrm{x}}$ network by coating it with various polymers, which are photon responsive over different parts of the solar spectrum.

- Generate a novel nanomaterial via the interaction of ultra-short laser pulses with a Titanium target coated with Silicon and investigate its photon absorption capability.

- Generate the multiphase nano $\mathrm{TiO}_{\mathrm{x}}$ material on a silicon wafer for easy integration into a photon harvesting device.

- Propose a method to design and fabricate a photon harvesting device by utilising the multiphase nano $\mathrm{TiO}_{\mathrm{x}}$ material as the photon absorbing material layer and measure performance characteristics of the device. 


\section{Appendices}

\section{A. Surface Temperature Calculation and Analysis}

In an attempt to understand when and where the formation of the multiphase Titanium Oxide on the phase functionalised titanium substrate described in Chapter 4 would take place, the thermal conditions caused by the ultrafast laser material interaction were simulated. First a theoretical study was conducted to calculate the final average surface temperature obtained at the centre of the laser-material interaction spot, by using the method and equations given in section 2.2. The laser absorption coefficient for Titanium at $1040 \mathrm{~nm}$ was taken to be 0.448 , thermal diffusivity as $0.090123 \mathrm{~cm}^{2} / \mathrm{s}$ and thermal conductivity as $0.219 \mathrm{~W} / \mathrm{cmK}$.

Matlab code was written for equation 3 given in Chapter 2 and executed to obtain a $\bar{T}_{\mathrm{n}}$ vs. $\mathrm{N}_{\text {eff }}$ graph shown in Figure 9.1. This figure shows the plots comparing the average surface temperature values (in Kelvin scale $[\mathrm{K}]$ ) for different laser pulse widths at a given laser fluence at the end of $\mathrm{N}_{\mathrm{eff}}$, the number of laser pulses. A significant increase in average surface temperatures was seen with an increase in laser fluence (Figure 9.1 (D)). This increase in temperature was seen to occur very rapidly in the first few pulses, after which the values remained more or less constant which implied that most of the energy transfer from the laser to the base metal substrate occurred during this time period. The plots also demonstrated that at constant laser fluence, increasing the pulse width results in a very minimal increase in temperature values $(<10 \mathrm{~K})$. 

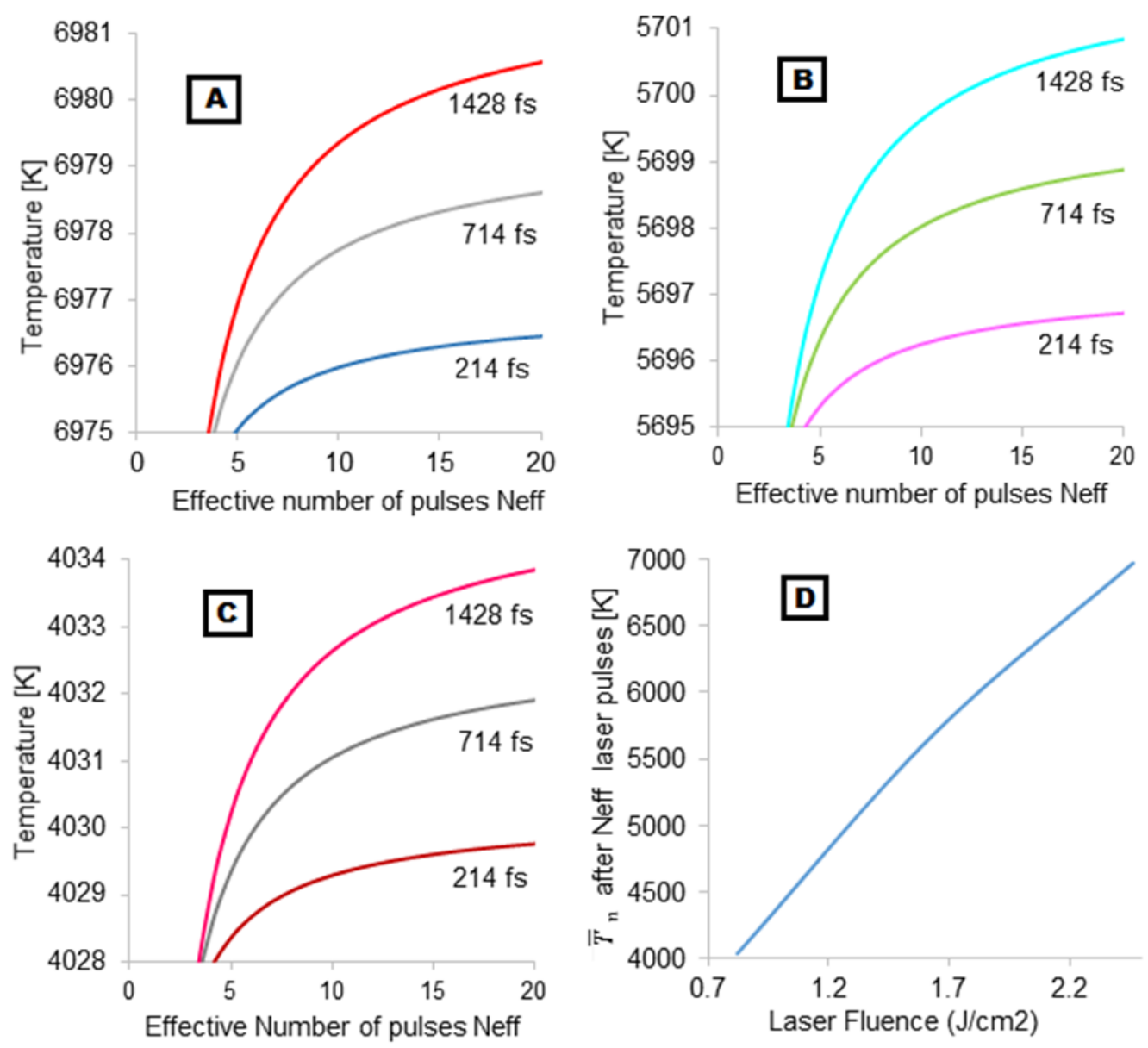

Figure 9.1: Avg. surface temp. calculated for the first twenty laser pulses at different fluence values:

(A) $2.4614 \mathrm{~J} / \mathrm{cm}^{2}$, (B) $1.6409 \mathrm{~J} / \mathrm{cm}^{2}$, (C) $0.8208 \mathrm{~J} / \mathrm{cm}^{2}$, and (D) variation of average surface temperature vs. laser fluence.

To further understand how this heat would dissipate away from the laser spot on the Titanium surface, another temperature analysis was conducted using Ansys Mechanical APDL software (version R14.5). The problem was reduced to a very simple 3D transient heat conduction model by ignoring the negligible radiation and convection effects and considering a rectangular block of Titanium with the following dimensions: $10 \mathrm{~mm} \times 10 \mathrm{~mm} \times 2 \mathrm{~mm}$, and the following material properties: thermal conductivity $0.219 \mathrm{~W} / \mathrm{cmK}$, specific heat $0.523 \mathrm{~J} / \mathrm{g}^{\circ} \mathrm{C}$, and density $4.15 \mathrm{~g} / \mathrm{cm}^{3}$. The element type selected was solid 90 and the entire area was meshed using the smart size mesh 
tool. For simplicity, the cumulative heating effect induced due to the scanning of the ultrafast laser pulse beam was ignored. As such, only a single laser-material interaction spot area was considered. A point heat source was introduced onto the surface of the Titanium block for a time period analogous to the actual laser material interaction time for a unit laser spot area. This point heat source was assumed to generate a heat equivalent to the average surface temperature value $\left(\bar{T}_{\mathrm{n}}\right)$ calculated earlier, at the end of $N_{\mathrm{eff}}$ number of laser pulses, at the center of the laser spot area. Subsequently the thermal heat diffusion along the surface and the depth of the Titanium block was modelled to generate the temperature profile at various distances from the laser spot centre after several time periods. The analysis was performed for the three laser fluence conditions $2.4614 \mathrm{~J} / \mathrm{cm}^{2}, 1.6409 \mathrm{~J} / \mathrm{cm}^{2}$ and $0.8208 \mathrm{~J} / \mathrm{cm}^{2}$ at a constant pulse width of $214 \mathrm{fs}$. The transient temperature profile at other pulse widths was not modelled due to minimal temperature difference at the end of $N_{\text {eff }}$ number of pulses, as seen in Figure 9.1 .

The contour plots in Figure 9.2 display the progression of thermal diffusion away from a single laser-material interaction spot area with time, for a laser fluence of $0.8208 \mathrm{~J} / \mathrm{cm}^{2}$. Similar contour plots were generated at other laser fluence values. To identify and explain the most probable Oxide phase of Titanium to have been formed at such thermal conditions, the Ti-O phase diagram was considered. 

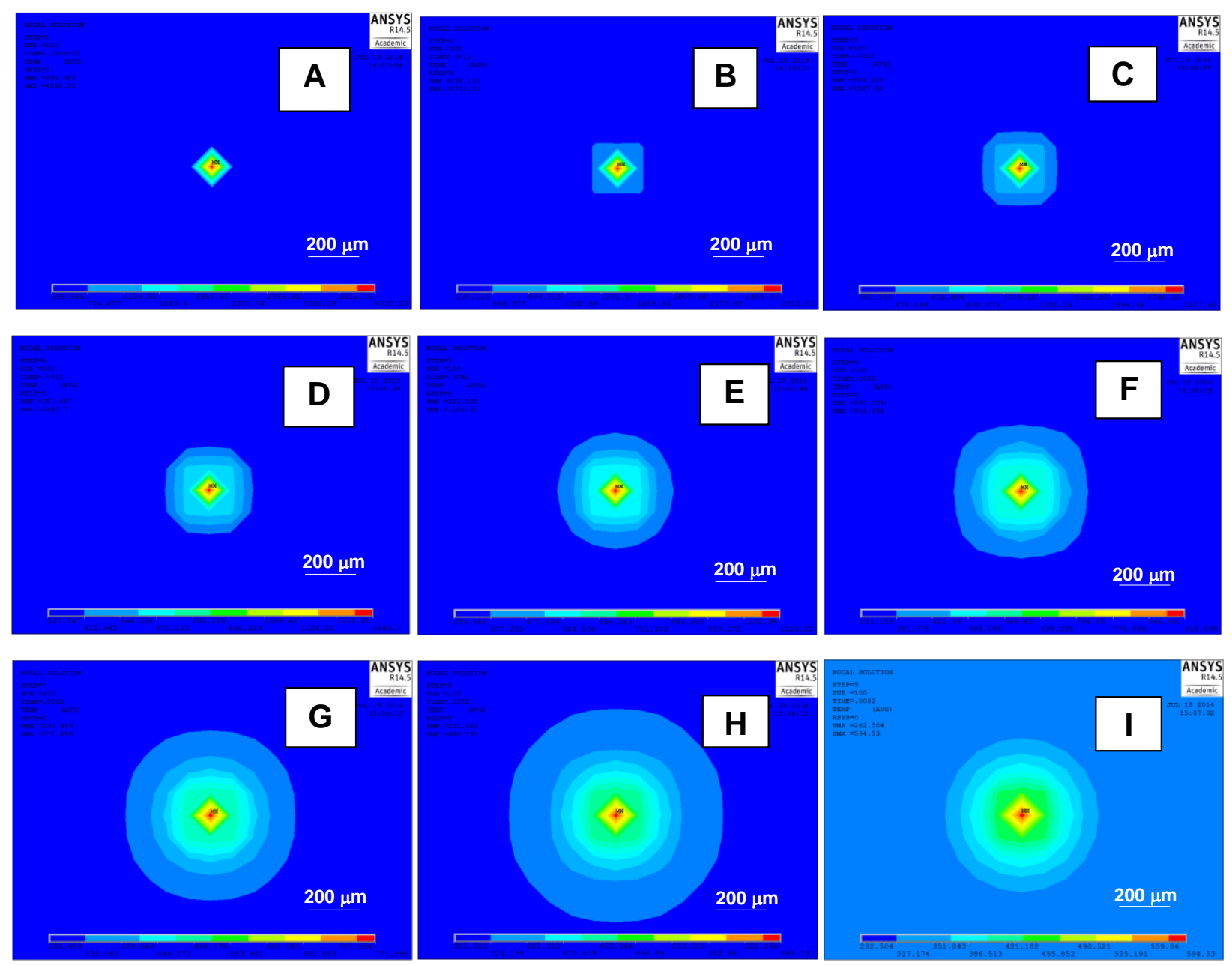

Figure 9.2: Thermal diffusion away from the laser material interaction spot for laser fluence of $0.8208 \mathrm{~J} / \mathrm{cm} 2$ right after the laser beam is removed (A) $0.2 \mathrm{~ms}$ and a few time periods after the end of the interaction, (B) $1 \mathrm{~ms}$, (C) $2 \mathrm{~ms}$, (D) $3 \mathrm{~ms}$, (E) $4 \mathrm{~ms}$, (F) $5 \mathrm{~ms}$, (G) $6 \mathrm{~ms}$, (H) $7 \mathrm{~ms}$, and (I) $8 \mathrm{~ms}$.

A careful observation of Ti-O phase diagram revealed the following about the three Oxides identified earlier via XRD analysis on the ultrafast laser transformed multi-phased Titanium Oxide zone [86]:

i. $\quad \mathrm{Ti}_{3} \mathrm{O}$ with a 25 atomic $\% \mathrm{O}$ is formed around about $673-773 \mathrm{~K}$.

ii. (TiO.716) 3.76 , having 42 atomic $\% \mathrm{O}$ is a structural modification of the high temperature Monoxide $\gamma \mathrm{TiO}$ and is formed around 1793-2053 K. 
iii. Rutile $\mathrm{TiO}_{2}$, stable at all temperatures, with a melting point of $2116 \mathrm{~K}$ is the richest O phase.

The oxidation of Titanium at such elevated temperatures by the laser beam would evidently lead to the diffusion of Oxygen atoms into the metal. The Oxygen diffusion length would also be longer at higher fluence. Analysis of the temperature profile generated by the transient 3D model was done, followed by its correlation with the Ti-O phase diagram. The solidification path $\mathrm{L}+(\alpha \mathrm{Ti}) \leftrightarrows \gamma \mathrm{TiO}$, observed in an Ti-O phase diagram, could have been taken by the molten metal leading to the formation of (TiO.716) 3.76 through the diffusion of Oxygen. The high Oxygen content rutile would mostly form near the extreme surface by the following phase transformation: $\mathrm{L} \leftrightarrows \mathrm{TiO}_{2}$ and at greater depths where diffusion of Oxygen is limited, the second order phase transition $(\alpha \mathrm{Ti}) \leftrightarrows \mathrm{Ti}_{2} \mathrm{O} \leftrightarrows \mathrm{Ti}_{3} \mathrm{O}$ could have been the likely path leading to the formation of $\mathrm{Ti}_{3} \mathrm{O}$.

The temperature values generated by the transient model on the sample surface have been plotted in Figure 9.3(A) and (B), up to a distance of about $200 \mu \mathrm{m}$ away from the laser centre spot, at various time intervals. Similar plots showing the temperature values at different depths along the laser spot centre are given in Figure 9.3(C) and (D).

A careful study of these plots showed that, the thermal conditions conducive for the formation of $\mathrm{Ti}_{3} \mathrm{O}$ at distances closer to the laser spot centre along the depth as well as on the surface, would develop only after about six to eight milliseconds post the end of the laser material interaction. It is however probable for $\mathrm{Ti}_{3} \mathrm{O}$ to have been formed much earlier, at greater depths, if adequate diffusion of Oxygen could have occurred by then. However, due to the inability of our diffractometer to study the composition at such longer depths, it could not be verified by XRD analysis. 

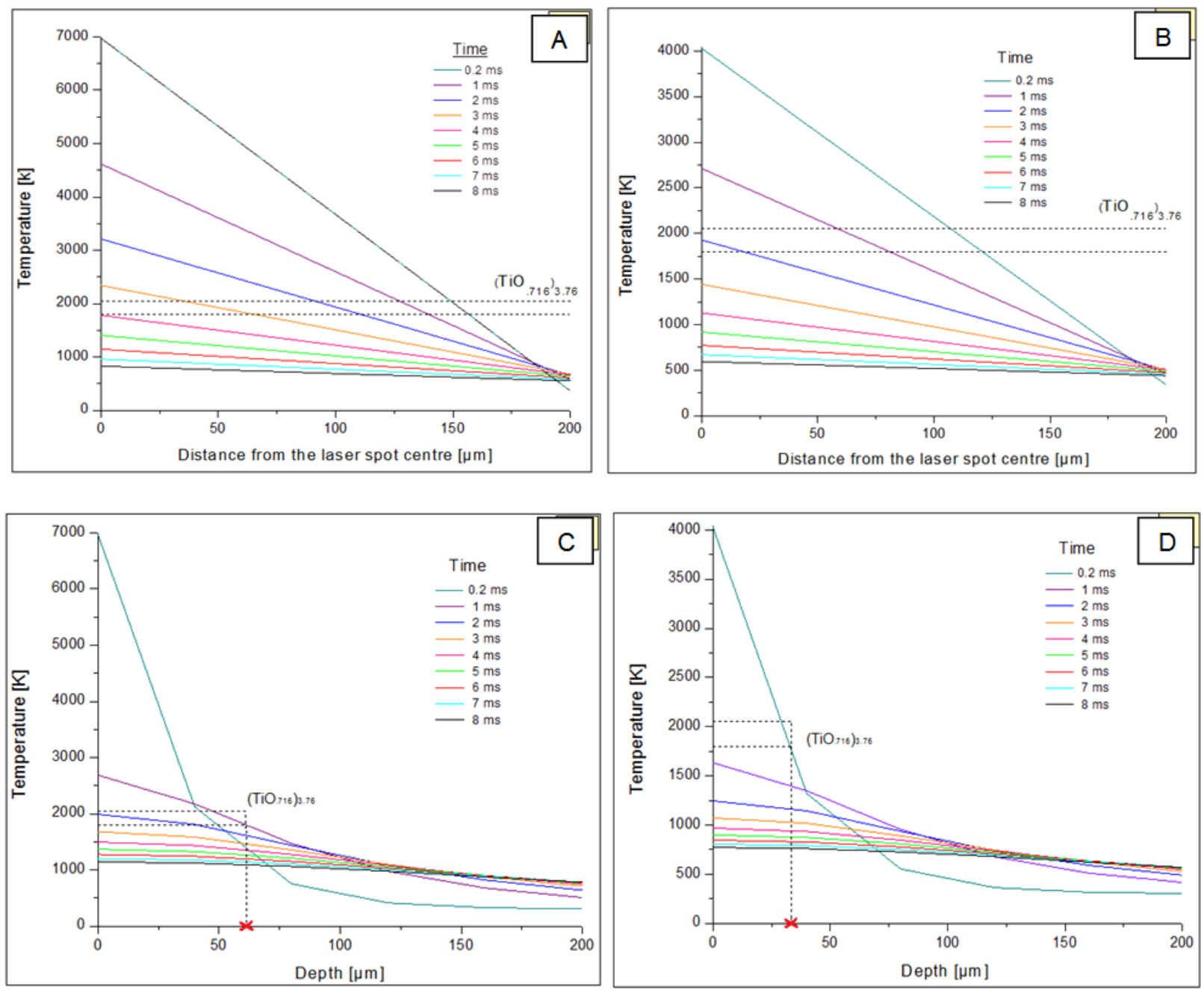

Figure 9.3: Temperature $[\mathrm{K}]$ vs. Distance from laser spot centre $[\mu \mathrm{m}]$ plot at laser fluence (A) $2.4614 \mathrm{~J} / \mathrm{cm}^{2}$, and (B) $0.8208 \mathrm{~J} / \mathrm{cm}^{2}$. The possible (TiO. $\left.{ }_{716}\right)_{3.76}$ formation temperature range is marked between the dotted lines in plot (A) and (B). Temperature [K] vs. Depth $[\mu \mathrm{m}]$ plot at laser fluence (C) $2.4614 \mathrm{~J} / \mathrm{cm}^{2}$ and (D) $0.8208 \mathrm{~J} / \mathrm{cm}^{2}$. The possible (TiO.716) 3.76 formation temperature range is indicated between the dotted lines in plot (C) and (D), with the extrapolated $\mathrm{x}$-intercept showing the maximum depth up to which it can form.

A plausible explanation for greater amount of $\mathrm{Ti}_{3} \mathrm{O}$ being formed at a higher fluence and shorter pulse widths (i.e. higher peak power), as determined by the Rietveld fitting, could be due to the presence of a much wider and deeper melt zone. The SEM image (B) given in the inset of Figure 4.5 clearly indicates more melting at a higher fluence value of $2.4614 \mathrm{~J} / \mathrm{cm}^{2}$, compared to lower 
fluence of $0.8208 \mathrm{~J} / \mathrm{cm}^{2}$ seen in image (A) of Figure 4.5. This accumulation of the melt zone having molten "platelets" as observed in the SEM images that could have caused a hindrance to the diffusion of Oxygen is due to higher laser fluency of pulses hitting the sample during laser interaction. However, further study is required to explain this occurrence.

As mentioned earlier, $\left(\mathrm{TiO}_{.716}\right)_{3.76}$ is formed around 1793-2053 K. This temperature zone has been marked in Figure 9.3 by dotted lines. On the surface, at regions closer than $100 \mu \mathrm{m}$ from the laser spot centre, it was seen that such temperature conditions develop sooner, that is $1 \mathrm{~ms}$ after the cessation of the laser material interaction, at a lower fluence $0.8208 \mathrm{~J} / \mathrm{cm}^{2}$. At higher fluence conditions of $2.4614 \mathrm{~J} / \mathrm{cm}^{2}$, it would take 2-3 ms longer. This could explain the presence of greater amount of (TiO.716) 3.76 being generated on samples at $0.8208 \mathrm{~J} / \mathrm{cm}^{2}$ laser fluence. Likewise, the marking of the temperature zone for the formation of (TiO.716)3.76 along the depth, shown in Figure 9.3(C) and (D) revealed that this non-stoichiometric Oxide could form up to a depth of $60 \mu \mathrm{m}$ at $2.4614 \mathrm{~J} / \mathrm{cm}^{2}$ laser fluence condition and only up to $30 \mu \mathrm{m}$ depth from the sample surface, at lower fluence of $0.8208 \mathrm{~J} / \mathrm{cm}^{2}$. However, it is very unlikely that Oxygen would diffuse to such depths, given the short (milliseconds) time scale.

\section{B. Calculation of Enhancement Factor}

The EF ratio was calculated by using the procedure and formula given by Cong. et al. [151]:

$$
\begin{gathered}
E F=\frac{I_{\text {hdd }} / N_{\text {surf }}}{I_{\text {sub }} / N_{\text {bulk }}} \\
N_{\text {surf }}=C V_{\text {Dye }} N_{\mathrm{A}} A_{\text {Raman }} / A_{\text {sub }}
\end{gathered}
$$




$$
N_{\text {bulk }}=M \rho h_{\mathrm{d}} N_{\mathrm{A}} A_{\mathrm{Raman}}
$$

where $I_{\text {hdd }}$ and $I_{\text {sub }}$ are the intensities of the characteristic Raman peak of the crystal violet (CV) dye when present on the HDD-nano $\mathrm{TiO}_{x}$ and bare Ti substrate respectively. $N_{\text {surf }}$ is the number of molecules contributing to the Raman signal from the HDD-nano $\mathrm{TiO}_{\mathrm{x}}$ and $N_{\text {bulk }}$ is the number of molecules contributing to the Raman signal from the Ti substrate. The values of $N_{\text {surf }}$ and $N_{\text {bulk }}$ values consider the interaction volume of the Raman laser with the dye molecules which contribute to the Raman enhancement. The data for $\mathrm{CV}$ dye on bare titanium substrate was used as reference for non-SERS active reference. It was assumed that the dye molecules were uniformly distributed on the substrate. $C$ is molar concentration of the dye $\left(10^{-3} \mathrm{M}\right), V_{\text {Dye }}$ is volume of dye droplet $(1 \mu \mathrm{l})$, $N_{\mathrm{A}}$ is the Avagadro constant. $A_{\text {Raman }}$ is Raman laser spot area (90 $\mu \mathrm{m}$ in diameter). $1 \mu \mathrm{l}$ of dye spread to a circle of $5 \mathrm{~mm}$ diameter on the sample substrate from which area of substrate, $A_{\text {sub }}$ was calculated. For calculation of $N_{\text {bulk }}$, half of depth of field $\left(h_{\mathrm{d}}\right)$ of laser beam $(10 / 2 \mu \mathrm{m})$ and CV dye molecular weight $(M)$ of $407.98 \mathrm{~g} / \mathrm{mol}$ and density $(\rho)$ of $0.981 \mathrm{~g} / \mathrm{ml}$ was used. 


\section{References}

[1] V. Ganapati, O. D. Miller, and E. Yablonovitch, "Light Trapping Textures Designed by Electromagnetic Optimization for Subwavelength Thick Solar Cells," IEEE Journal of Photovoltaics, vol. 4, no. 1, pp. 175-182, Jan. 2014.

[2] Z. Liu et al., "Automatically Acquired Broadband Plasmonic-Metamaterial Black Absorber during the Metallic Film-Formation," ACS Appl. Mater. Interfaces, vol. 7, no. 8, pp. 49624968, Mar. 2015.

[3] J. Sun, L. Liu, G. Dong, and J. Zhou, "An extremely broad band metamaterial absorber based on destructive interference," Opt. Express, OE, vol. 19, no. 22, pp. 21155-21162, Oct. 2011.

[4] M. K. Hedayati et al., "Design of a perfect black absorber at visible frequencies using plasmonic metamaterials," Adv. Mater. Weinheim, vol. 23, no. 45, pp. 5410-5414, Dec. 2011.

[5] M. A. Kats, R. Blanchard, P. Genevet, and F. Capasso, "Nanometre optical coatings based on strong interference effects in highly absorbing media," Nat Mater, vol. 12, no. 1, pp. 2024, Jan. 2013.

[6] N. Bloembergen, "Solid state infrared quantum counters," Physical Review Letters, vol. 2, no. 3, pp. 84-85, 1959.

[7] F. Auzel, "Upconversion and Anti-Stokes Processes with f and d Ions in Solids," Chemical Reviews, vol. 104, no. 1, pp. 139-173, 2004.

[8] G. Chen, J. Seo, C. Yang, and P. N. Prasad, "Nanochemistry and nanomaterials for photovoltaics," Chem. Soc. Rev., vol. 42, no. 21, pp. 8304-8338, Oct. 2013. 
[9] N. Asim et al., "A review on the role of materials science in solar cells," Renewable and Sustainable Energy Reviews, vol. 16, no. 8, pp. 5834-5847, 2012.

[10] L. Tsakalakos, "Nanostructures for photovoltaics," Materials Science and Engineering: R: Reports, vol. 62, no. 6, pp. 175-189, 2008.

[11] A. Fujishima and K. Honda, "Electrochemical photolysis of water at a semiconductor electrode," Nature, vol. 238, no. 5358, pp. 37-38, 1972.

[12] M. Pelaez et al., "A review on the visible light active titanium dioxide photocatalysts for environmental applications," Applied Catalysis B: Environmental, vol. 125, pp. 331-349, 2012.

[13] A. Wisitsoraat, A. Tuantranont, E. Comini, G. Sberveglieri, and W. Wlodarski, "Characterization of n-type and p-type semiconductor gas sensors based on NiOx doped TiO2 thin films," Thin Solid Films, vol. 517, no. 8, pp. 2775-2780, Feb. 2009.

[14] X. Chen and S. S. Mao, "Titanium dioxide nanomaterials: synthesis, properties, modifications, and applications," Chemical reviews, vol. 107, no. 7, pp. 2891-2959, 2007.

[15] X. Chen and S. S. Mao, "Synthesis of titanium dioxide (TiO2) nanomaterials.," Journal of nanoscience and nanotechnology, vol. 6, no. 4, pp. 906-925, 2006.

[16] P. V. Kamat, “TiO2 Nanostructures: Recent Physical Chemistry Advances,” J. Phys. Chem. C, vol. 116, no. 22, pp. 11849-11851, Jun. 2012.

[17] M. Montazer and S. Seifollahzadeh, "Enhanced Self-cleaning, Antibacterial and UV Protection Properties of Nano TiO2 Treated Textile through Enzymatic Pretreatment," Photochemistry and Photobiology, vol. 87, no. 4, pp. 877-883, Jul. 2011. 
[18] H. Yang, S. Zhu, and N. Pan, "Studying the mechanisms of titanium dioxide as ultravioletblocking additive for films and fabrics by an improved scheme," J. Appl. Polym. Sci., vol. 92, no. 5, pp. 3201-3210, Jun. 2004.

[19] J. H. Braun, A. Baidins, and R. E. Marganski, "TiO2 pigment technology: a review," Progress in Organic Coatings, vol. 20, no. 2, pp. 105-138, May 1992.

[20] M. J. Gázquez, J. P. Bolívar, R. Garcia-Tenorio, and F. Vaca, "A Review of the Production Cycle of Titanium Dioxide Pigment," Materials Sciences and Applications, vol. 05, no. 07, p. 441, May 2014.

[21] M. M. Ba-Abbad, A. A. H. Kadhum, A. B. Mohamad, M. S. Takriff, and K. Sopian, "Synthesis and catalytic activity of $\mathrm{TiO} 2$ nanoparticles for photochemical oxidation of concentrated chlorophenols under direct solar radiation," Int. J. Electrochem. Sci, vol. 7, pp. 4871-4888, 2012.

[22] X. CHEN, "Titanium dioxide nanomaterials and their energy applications," Chinese Journal of Catalysis, vol. 30, no. 8, pp. 839-851, 2009.

[23] S. Banerjee, D. D. Dionysiou, and S. C. Pillai, "Self-cleaning applications of TiO2 by photoinduced hydrophilicity and photocatalysis," Applied Catalysis B: Environmental, vol. 176177, pp. 396-428, Oct. 2015.

[24] F. Zhang, J. Zhao, T. Shen, H. Hidaka, E. Pelizzetti, and N. Serpone, "TiO2-assisted photodegradation of dye pollutants II. Adsorption and degradation kinetics of eosin in TiO2 dispersions under visible light irradiation," Applied Catalysis B: Environmental, vol. 15, no. 1-2, pp. 147-156, Jan. 1998.

[25] R. Vinu and G. Madras, "Kinetics of Sonophotocatalytic Degradation of Anionic Dyes with Nano-TiO2,” Environ. Sci. Technol., vol. 43, no. 2, pp. 473-479, Jan. 2009. 
[26] P.-C. Maness, S. Smolinski, D. M. Blake, Z. Huang, E. J. Wolfrum, and W. A. Jacoby, "Bactericidal Activity of Photocatalytic TiO2 Reaction: toward an Understanding of Its Killing Mechanism,” Appl. Environ. Microbiol., vol. 65, no. 9, pp. 4094-4098, Sep. 1999.

[27] C. Burda, Y. Lou, X. Chen, A. C. S. Samia, J. Stout, and J. L. Gole, "Enhanced Nitrogen Doping in TiO2 Nanoparticles," Nano Lett., vol. 3, no. 8, pp. 1049-1051, Aug. 2003.

[28] X. Zhang, S.-T. Wang, and Z.-S. Wang, "Effect of metal-doping in TiO2 on fill factor of dyesensitized solar cells," Applied Physics Letters, vol. 99, no. 11, p. 113503, Sep. 2011.

[29] S. Bingham and W. A. Daoud, "Recent advances in making nano-sized TiO2 visible-light active through rare-earth metal doping," J. Mater. Chem., vol. 21, no. 7, pp. 2041-2050, Feb. 2011.

[30] B. Liu, H. M. Chen, C. Liu, S. C. Andrews, C. Hahn, and P. Yang, "Large-Scale Synthesis of Transition-Metal-Doped TiO2 Nanowires with Controllable Overpotential," J. Am. Chem. Soc., vol. 135, no. 27, pp. 9995-9998, Jul. 2013.

[31] D. Chen, Z. Jiang, J. Geng, Q. Wang, and D. Yang, "Carbon and Nitrogen Co-doped TiO2 with Enhanced Visible-Light Photocatalytic Activity," Ind. Eng. Chem. Res., vol. 46, no. 9, pp. 2741-2746, Apr. 2007.

[32] G. Wu, T. Nishikawa, B. Ohtani, and A. Chen, "Synthesis and Characterization of CarbonDoped TiO2 Nanostructures with Enhanced Visible Light Response,” Chem. Mater., vol. 19, no. 18, pp. 4530-4537, Sep. 2007.

[33] E. Wang, T. He, L. Zhao, Y. Chen, and Y. Cao, "Improved visible light photocatalytic activity of titania doped with tin and nitrogen," Journal of Materials Chemistry, vol. 21, no. 1, pp. 144-150, 2011. 
[34] C. Fan, P. Xue, and Y. Sun, "Preparation of Nano-TiO2 Doped with Cerium and Its Photocatalytic Activity," Journal of Rare Earths, vol. 24, no. 3, pp. 309-313, Jun. 2006.

[35] K. Gupta, R. P. Singh, A. Pandey, and A. Pandey, "Photocatalytic antibacterial performance of TiO2 and Ag-doped TiO2 against S. aureus. P. aeruginosa and E. coli," Beilstein Journal of Nanotechnology, vol. 4, no. 1, pp. 345-351, Jun. 2013.

[36] T.-D. Nguyen-Phan et al., "Three-dimensional ruthenium-doped TiO 2 sea urchins for enhanced visible-light-responsive H 2 production," Physical Chemistry Chemical Physics, vol. 18 , no. 23 , pp. 15972-15979, 2016.

[37] K. M. Rahulan, S. Ganesan, and P. Aruna, "Synthesis and optical limiting studies of Audoped TiO 2 nanoparticles," Adv. Nat. Sci: Nanosci. Nanotechnol., vol. 2, no. 2, p. 025012, 2011.

[38] S. Mathew et al., "Dye-sensitized solar cells with $13 \%$ efficiency achieved through the molecular engineering of porphyrin sensitizers," Nat Chem, vol. 6, no. 3, pp. 242-247, Mar. 2014.

[39] "Near-infrared sensitization of solid-state dye-sensitized solar cells with a squaraine dye," Appl. Phys. Lett., vol. 100, no. 17, p. 173512, Apr. 2012.

[40] "Photovoltaic cells made from conjugated polymers infiltrated into mesoporous titania," Appl. Phys. Lett., vol. 83, no. 16, pp. 3380-3382, Oct. 2003.

[41] J. Kuendig, M. Goetz, A. Shah, L. Gerlach, and E. Fernandez, "Thin film silicon solar cells for space applications: Study of proton irradiation and thermal annealing effects on the characteristics of solar cells and individual layers," Solar energy materials and solar cells, vol. 79, no. 4, pp. 425-438, 2003. 
[42] P. Yu, K. Zhu, A. G. Norman, S. Ferrere, A. J. Frank, and A. J. Nozik, "Nanocrystalline TiO2 Solar Cells Sensitized with InAs Quantum Dots," J. Phys. Chem. B, vol. 110, no. 50, pp. 25451-25454, Dec. 2006.

[43] N. Guijarro, T. Lana-Villarreal, T. Lutz, S. A. Haque, and R. Gómez, "Sensitization of TiO2 with PbSe Quantum Dots by SILAR: How Mercaptophenol Improves Charge Separation,” J. Phys. Chem. Lett., vol. 3, no. 22, pp. 3367-3372, Nov. 2012.

[44] S. Chaguetmi et al., "Photocatalytic activity of $\mathrm{TiO} 2$ nanofibers sensitized with $\mathrm{ZnS}$ quantum dots," RSC Advances, vol. 3, no. 8, p. 2572, 2013.

[45] H. Zhang, X. Quan, S. Chen, H. Yu, and N. Ma, “'Mulberry-like' CdSe Nanoclusters Anchored on TiO2 Nanotube Arrays: A Novel Architecture with Remarkable Photoelectrochemical Performance," Chem. Mater., vol. 21, no. 14, pp. 3090-3095, Jul. 2009.

[46] Y.-C. Pu et al., "Au Nanostructure-Decorated TiO2 Nanowires Exhibiting Photoactivity Across Entire UV-visible Region for Photoelectrochemical Water Splitting," Nano Lett., vol. 13, no. 8, pp. 3817-3823, Aug. 2013.

[47] I. Tanahashi, F. Yamazaki, and K. Hamada, "Localized Surface Plasmon Resonance Sensing Properties of Ag/TiO2 Films," Chem. Lett., vol. 35, no. 4, pp. 454-455, Mar. 2006.

[48] Z. H. Shah et al., "Highly enhanced plasmonic photocatalytic activity of $\mathrm{Ag} / \mathrm{AgCl} / \mathrm{TiO} 2$ by CuO co-catalyst," J. Mater. Chem. A, vol. 3, no. 7, pp. 3568-3575, Feb. 2015.

[49] X. Wan, T. Wang, Y. Dong, and D. He, "Development and Application of TiO2 Nanoparticles Coupled with Silver Halide," Journal of Nanomaterials, vol. 2014, p. e908785, Nov. 2014. 
[50] Z. Zhang, L. Zhang, M. N. Hedhili, H. Zhang, and P. Wang, "Plasmonic gold nanocrystals coupled with photonic crystal seamlessly on $\mathrm{TiO} 2$ nanotube photoelectrodes for efficient visible light photoelectrochemical water splitting," Nano letters, vol. 13, no. 1, pp. 14-20, 2012.

[51] N. C. Jeong, C. Prasittichai, and J. T. Hupp, "Photocurrent Enhancement by Surface Plasmon Resonance of Silver Nanoparticles in Highly Porous Dye-Sensitized Solar Cells," Langmuir, vol. 27, no. 23, pp. 14609-14614, Dec. 2011.

[52] H. Yan, S. T. Kochuveedu, L. N. Quan, S. S. Lee, and D. H. Kim, "Enhanced photocatalytic activity of C, F-codoped TiO2 loaded with AgCl," Journal of Alloys and Compounds, vol. 560, pp. 20-26, May 2013.

[53] M. K. Nowotny, T. Bak, and J. Nowotny, "Electrical Properties and Defect Chemistry of TiO2 Single Crystal. I. Electrical Conductivity,” J. Phys. Chem. B, vol. 110, no. 33, pp. 16270-16282, Aug. 2006.

[54] X. Chen et al., "Properties of Disorder-Engineered Black Titanium Dioxide Nanoparticles through Hydrogenation,” Scientific Reports, vol. 3, Mar. 2013.

[55] J.-Y. Shin, J. H. Joo, D. Samuelis, and J. Maier, “Oxygen-Deficient TiO2- $\delta$ Nanoparticles via Hydrogen Reduction for High Rate Capability Lithium Batteries," Chem. Mater., vol. 24, no. 3, pp. 543-551, Feb. 2012.

[56] Y. H. Hu, "A Highly Efficient Photocatalyst-Hydrogenated Black TiO2 for the Photocatalytic Splitting of Water," Angew. Chem. Int. Ed., vol. 51, no. 50, pp. 12410-12412, Dec. 2012. 
[57] X. Liu, G. Zhu, X. Wang, X. Yuan, T. Lin, and F. Huang, "Progress in Black Titania: A New Material for Advanced Photocatalysis," Adv. Energy Mater., vol. 6, no. 17, p. n/a-n/a, Sep. 2016.

[58] X. Pan, M.-Q. Yang, X. Fu, N. Zhang, and Y.-J. Xu, "Defective TiO2 with oxygen vacancies: synthesis, properties and photocatalytic applications," Nanoscale, vol. 5, no. 9, pp. 36013614, Apr. 2013.

[59] T. Lin et al., "Effective nonmetal incorporation in black titania with enhanced solar energy utilization,” Energy \& Environmental Science, vol. 7, no. 3, pp. 967-972, 2014.

[60] M. A. Henderson, "A surface science perspective on photocatalysis," Surface Science Reports, vol. 66, no. 6, pp. 185-297, 2011.

[61] X. Liu et al., "Green synthetic approach for Ti3+ self-doped $\mathrm{TiO} 2-\mathrm{x}$ nanoparticles with efficient visible light photocatalytic activity," Nanoscale, vol. 5, no. 5, pp. 1870-1875, Feb. 2013.

[62] M. Qiu et al., "Synthesis of Ti3+ self-doped TiO2 nanocrystals based on Le Chatelier's principle and their application in solar light photocatalysis," $R S C A d v$., vol. 6, no. 78, pp. 74376-74383, Aug. 2016.

[63] Q. Zhu et al., "Stable blue TiO2-x nanoparticles for efficient visible light photocatalysts," $J$. Mater. Chem. A, vol. 2, no. 12, pp. 4429-4437, Feb. 2014.

[64] M. W. Shah et al., "Facile Synthesis of Defective TiO2-x Nanocrystals with High Surface Area and Tailoring Bandgap for Visible-light Photocatalysis," Scientific Reports, vol. 5, p. 15804, Oct. 2015.

[65] J. Dong et al., "Defective Black TiO2 Synthesized via Anodization for Visible-Light Photocatalysis," ACS Appl. Mater. Interfaces, vol. 6, no. 3, pp. 1385-1388, Feb. 2014. 
[66] A. Naldoni et al., "Effect of Nature and Location of Defects on Bandgap Narrowing in Black TiO2 Nanoparticles,” J. Am. Chem. Soc., vol. 134, no. 18, pp. 7600-7603, May 2012.

[67] X. Chen, L. Liu, P. Y. Yu, and S. S. Mao, "Increasing Solar Absorption for Photocatalysis with Black Hydrogenated Titanium Dioxide Nanocrystals," Science, vol. 331, no. 6018, pp. 746-750, Feb. 2011.

[68] X. Chen, L. Liu, and F. Huang, "Black titanium dioxide (TiO2 ) nanomaterials," Chemical Society Reviews, vol. 44, no. 7, pp. 1861-1885, 2015.

[69] D. M. Gates, "Spectral distribution of solar radiation at the earth's surface," Science, vol. 151, no. 3710, pp. 523-529, 1966.

[70] M. Sivayoganathan, "Particle agglomerated 3-d nanostructures for photon absorption," Ph.D. Thesis, Ryerson University, 2013.

[71] M. S. Brown and C. B. Arnold, "Fundamentals of Laser-Material Interaction and Application to Multiscale Surface Modification," in Laser Precision Microfabrication, K. Sugioka, M. Meunier, and A. Piqué, Eds. Springer Berlin Heidelberg, 2010, pp. 91-120.

[72] S. Eliezer et al., "Synthesis of nanoparticles with femtosecond laser pulses," Phys. Rev. B, vol. 69 , no. 14, p. 144119, Apr. 2004.

[73] C. W. Schneider and T. Lippert, "Laser Ablation and Thin Film Deposition," in Laser Processing of Materials, P. Schaaf, Ed. Springer Berlin Heidelberg, 2010, pp. 89-112.

[74] B. Tan, A. Dalili, and K. Venkatakrishnan, "High repetition rate femtosecond laser nanomachining of thin films," Applied Physics A: Materials Science and Processing, vol. 95, no. 2, pp. 537-545, 2009. 
[75] E. G. Gamaly, A. V. Rode, and B. Luther-Davies, "Ultrafast ablation with high-pulse-rate lasers. Part I: Theoretical considerations," Journal of Applied Physics, vol. 85, no. 8 I, pp. $4213-4221,1999$.

[76] S. Panchatsharam, B. Tan, and K. Venkatakrishnan, "Femtosecond laser-induced shockwave formation on ablated silicon surface," Journal of Applied Physics, vol. 105, no. 9, p. 093103, May 2009.

[77] A. Kiani, K. Venkatakrishnan, and B. Tan, "Enhancement of the optical absorption of thinfilm of amorphorized silicon for photovoltaic energy conversion,” Solar Energy, vol. 85, no. 9, pp. 1817-1823, 2011.

[78] C. Liu, "A study of particle generation during laser ablation with applications," Lawrence Berkeley National Laboratory, Aug. 2005.

[79] V. Piñon and D. Anglos, "Optical emission studies of plasma induced by single and double femtosecond laser pulses," Spectrochimica Acta Part B: Atomic Spectroscopy, vol. 64, no. 10, pp. 950-960, Oct. 2009.

[80] D. Bleiner, "Mathematical modelling of laser-induced particulate formation in direct solid microanalysis," Spectrochimica Acta Part B: Atomic Spectroscopy, vol. 60, no. 1, pp. 49-64, Jan. 2005.

[81] B. S. Luk'yanchuk, W. Marine, S. I. Anisimov, and G. A. Simakina, "Condensation of vapor and nanoclusters formation within the vapor plume, produced by ns-laser ablation of $\mathrm{Si}$, $\mathrm{Ge}$ and C," presented at the Proceedings of SPIE - The International Society for Optical Engineering, 1999, vol. 3618, pp. 434-452. 
[82] S. Senadheera, B. Tan, and K. Venkatakrishnan, "Critical Time to Nucleation: Graphite and Silicon Nanoparticle Generation by Laser Ablation," Journal of Nanotechnology, vol. 2009, p. e590763, Feb. 2009.

[83] B. R. Tull, J. E. Carey, M. A. Sheehy, C. Friend, and E. Mazur, "Formation of silicon nanoparticles and web-like aggregates by femtosecond laser ablation in a background gas," Appl. Phys. A, vol. 83, no. 3, pp. 341-346, Jun. 2006.

[84] M. Kauf, R. Patel, and J. Bovatsek, "High Speed Processing for Microelectronics and Photovoltaics," LTJ, vol. 6, no. 1, pp. 33-37, Jan. 2009.

[85] A. Faghri and Y. Zhang, Transport Phenomena in Multiphase Systems. Academic Press, 2006.

[86] J. L. Murray and H. A. Wriedt, “The O-Ti (Oxygen-Titanium) system,” JPE, vol. 8, no. 2, pp. 148-165, Apr. 1987.

[87] S. Andersson et al., "Phase Analysis Studies on the Titanium-Oxygen System.," Acta Chemica Scandinavica, vol. 11, pp. 1641-1652, 1957.

[88] A. Jostsons and A. S. Malin, "The ordered structure of Ti3O," Acta Crystallographica Section B Structural Crystallography and Crystal Chemistry, vol. 24, no. 2, pp. 211-213, Feb. 1968.

[89] J. Tauc and A. Menth, "States in the gap," Journal of Non-Crystalline Solids, vol. 8-10, no. C, pp. 569-585, 1972.

[90] J. Wang et al., "Oxygen Vacancy Induced Band-Gap Narrowing and Enhanced Visible Light Photocatalytic Activity of ZnO,” ACS Appl. Mater. Interfaces, vol. 4, no. 8, pp. 4024-4030, Aug. 2012. 
[91] E. G. Gamaly, A. V. Rode, B. Luther-Davies, and V. T. Tikhonchuk, "Ablation of solids by femtosecond lasers: Ablation mechanism and ablation thresholds for metals and dielectrics," Physics of Plasmas (1994-present), vol. 9, no. 3, pp. 949-957, Mar. 2002.

[92] M. Sivayoganathan, B. Tan, and K. Venkatakrishnan, "Formation of ring-patterned nanoclusters by laser-plume interaction," J Nanopart Res, vol. 15, no. 1, pp. 1-9, Jan. 2013.

[93] M. Ullmann, S. K. Friedlander, and A. Schmidt-Ott, "Nanoparticle Formation by Laser Ablation," Journal of Nanoparticle Research, vol. 4, no. 6, pp. 499-509, Dec. 2002.

[94] M. Sivayoganathan, B. Tan, and K. Venkatakrishnan, "Synthesis of crystalline and amorphous, particle-agglomerated 3-D nanostructures of $\mathrm{Al}$ and $\mathrm{Si}$ oxides by femtosecond laser and the prediction of these particle sizes," Nanoscale Res Lett, vol. 7, no. 1, pp. 1-7, Dec. 2012.

[95] D. O. Scanlon et al., "Band alignment of rutile and anatase TiO2," Nat Mater, vol. 12, no. 9, pp. 798-801, Sep. 2013.

[96] H. Zhang and J. F. Banfield, "Kinetics of Crystallization and Crystal Growth of Nanocrystalline Anatase in Nanometer-Sized Amorphous Titania," Chem. Mater., vol. 14, no. 10 , pp. 4145-4154, Oct. 2002.

[97] E. Smith and G. Dent, Modern Raman spectroscopy: a practical approach. J. Wiley, 2005.

[98] J. G. Smith, J. A. Faucheaux, and P. K. Jain, "Plasmon resonances for solar energy harvesting: A mechanistic outlook," Nano Today, vol. 10, no. 1, pp. 67-80, Feb. 2015.

[99] Y. Lin et al., "Dual-Layer Nanostructured Flexible Thin-Film Amorphous Silicon Solar Cells with Enhanced Light Harvesting and Photoelectric Conversion Efficiency," ACS Applied Materials \& Interfaces, vol. 8, no. 17, pp. 10929-10936, May 2016. 
[100] H. A. Atwater and A. Polman, "Plasmonics for improved photovoltaic devices," Nat Mater, vol. 9, no. 3, pp. 205-213, Mar. 2010.

[101] W. Raja et al., "Broadband absorption enhancement in plasmonic nanoshells-based ultrathin microcrystalline-Si solar cells," Scientific Reports, vol. 6, p. 24539, Apr. 2016.

[102] D. A. Hines and P. V. Kamat, "Recent Advances in Quantum Dot Surface Chemistry," ACS Applied Materials \& Interfaces, vol. 6, no. 5, pp. 3041-3057, Mar. 2014.

[103] M. R. Kim and D. Ma, "Quantum-Dot-Based Solar Cells: Recent Advances, Strategies, and Challenges," J. Phys. Chem. Lett., vol. 6, no. 1, pp. 85-99, Jan. 2015.

[104] L. Kavan, T. Stoto, M. Graetzel, D. Fitzmaurice, and V. Shklover, "Quantum size effects in nanocrystalline semiconducting titania layers prepared by anodic oxidative hydrolysis of titanium trichloride," The Journal of Physical Chemistry, vol. 97, no. 37, pp. 9493-9498, 1993.

[105] S. Pillai, K. R. Catchpole, T. Trupke, and M. A. Green, "Surface plasmon enhanced silicon solar cells," Journal of Applied Physics, vol. 101, no. 9, p. 093105, May 2007.

[106] T. Sugaya et al., "Highly stacked and well-aligned In0.4Ga0.6As quantum dot solar cells with In0.2Ga0.8As cap layer," Applied Physics Letters, vol. 97, no. 18, p. 183104, Nov. 2010.

[107] T. Sugaya, Y. Kamikawa, S. Furue, T. Amano, M. Mori, and S. Niki, "Multi-stacked quantum dot solar cells fabricated by intermittent deposition of InGaAs," Solar Energy Materials and Solar Cells, vol. 95, no. 1, pp. 163-166, Jan. 2011.

[108] J. Geldmeier, T. König, M. A. Mahmoud, M. A. El-Sayed, and V. V. Tsukruk, "Tailoring the Plasmonic Modes of a Grating-Nanocube Assembly to Achieve Broadband Absorption in the Visible Spectrum," Advanced Functional Materials, vol. 24, no. 43, pp. 6797-6805, Nov. 2014. 
[109] C.-T. Ko, Y.-Y. Han, W.-C. Wang, J. Shieh, and M.-J. Chen, "Enhancement of Light Emission from Silicon by Precisely Tuning Coupled Localized Surface Plasmon Resonance of a Nanostructured Platinum Layer Prepared by Atomic Layer Deposition," ACS Applied Materials \& Interfaces, vol. 6, no. 6, pp. 4179-4185, Mar. 2014.

[110] V. Amendola, R. Saija, O. M. Maragò, and M. A. Iatì, "Superior plasmon absorption in iron-doped gold nanoparticles," Nanoscale, vol. 7, no. 19, pp. 8782-8792, May 2015.

[111] G. G. Paulson and A. L. Friedberg, "Coalescence and agglomeration of gold films," Thin Solid Films, vol. 5, no. 1, pp. 47-52, Jan. 1970.

[112] “SC7620 Mini Sputter Coater Operating Manual.” Quorum Technologies.

[113] V. H. Grassian, "When Size Really Matters: Size-Dependent Properties and Surface Chemistry of Metal and Metal Oxide Nanoparticles in Gas and Liquid Phase Environments†," J. Phys. Chem. C, vol. 112, no. 47, pp. 18303-18313, Nov. 2008.

[114] K.-S. Lee and M. A. El-Sayed, "Gold and Silver Nanoparticles in Sensing and Imaging: Sensitivity of Plasmon Response to Size, Shape, and Metal Composition,” J. Phys. Chem. B, vol. 110, no. 39, pp. 19220-19225, Oct. 2006.

[115] P. Thakur, B. Tan, and K. Venkatakrishnan, "Multiphase titanium oxide nanomaterial for augmented vis-NIR photon absorption," Solar Energy Materials and Solar Cells, vol. 152, pp. 161-169, Aug. 2016.

[116] M. V. Artemyev, U. Woggon, H. Jaschinski, L. I. Gurinovich, and S. V. Gaponenko, "Spectroscopic Study of Electronic States in an Ensemble of Close-Packed CdSe Nanocrystals," J. Phys. Chem. B, vol. 104, no. 49, pp. 11617-11621, Dec. 2000. 
[117] A. Furube, L. Du, K. Hara, R. Katoh, and M. Tachiya, "Ultrafast Plasmon-Induced Electron Transfer from Gold Nanodots into TiO2 Nanoparticles,” J. Am. Chem. Soc., vol. 129, no. 48, pp. 14852-14853, Dec. 2007.

[118] C. Noguez, "Optical properties of isolated and supported metal nanoparticles," Optical Materials, vol. 27, no. 7, pp. 1204-1211, Apr. 2005.

[119] M. Schmid, R. Klenk, M. C. Lux-Steiner, M. Topic, and J. Krc, "Modeling plasmonic scattering combined with thin-film optics," Nanotechnology, vol. 22, no. 2, p. 025204, Jan. 2011.

[120] K. R. Catchpole and A. Polman, "Plasmonic solar cells," Optics Express, vol. 16, no. 26, p. 21793 , Dec. 2008.

[121] J. Z. Zhang, Optical Properties and Spectroscopy of Nanomaterials. World Scientific, 2009.

[122] H. Perron et al., "Combined investigation of water sorption on TiO2 rutile (lll 110$)$ single crystal face: XPS vs. periodic DFT,” Surface Science, vol. 601, no. 2, pp. 518-527, Jan. 2007.

[123] D. J. Mowbray, J. I. Martinez, J. M. García Lastra, K. S. Thygesen, and K. W. Jacobsen, "Stability and Electronic Properties of TiO2 Nanostructures With and Without B and N Doping," J. Phys. Chem. C, vol. 113, no. 28, pp. 12301-12308, Jul. 2009.

[124] H. Li, Y. Guo, and J. Robertson, "Calculation of TiO2 Surface and Subsurface Oxygen Vacancy by the Screened Exchange Functional," J. Phys. Chem. C, vol. 119, no. 32, pp. 18160-18166, Aug. 2015.

[125] P. K. Barhai, N. Kumari, I. Banerjee, S. K. Pabi, and S. K. Mahapatra, "Study of the effect of plasma current density on the formation of titanium nitride and titanium oxynitride thin 
films prepared by reactive DC magnetron sputtering," Vacuum, vol. 84, no. 7, pp. 896-901, Mar. 2010.

[126] L.-B. Xiong, J.-L. Li, B. Yang, and Y. Yu, "Ti3+ in the Surface of Titanium Dioxide: Generation, Properties and Photocatalytic Application," Journal of Nanomaterials, vol. 2012, Jan. 2012.

[127] X. Zhang et al., "The role of oxygen vacancy-Ti3+ states on TiO2 nanotubes' surface in dye-sensitized solar cells," Materials Letters, vol. 100, pp. 51-53, Jun. 2013.

[128] J. Ovenstone and K. Yanagisawa, "Effect of Hydrothermal Treatment of Amorphous Titania on the Phase Change from Anatase to Rutile during Calcination," Chem. Mater., vol. 11, no. 10, pp. 2770-2774, Oct. 1999.

[129] J. I. Langford and A. J. C. Wilson, "Scherrer after sixty years: A survey and some new results in the determination of crystallite size," J Appl Cryst, J Appl Crystallogr, vol. 11, no. 2, pp. 102-113, Apr. 1978.

[130] P. Scherrer, "Bestimmung der inneren Struktur und der Größe von Kolloidteilchen mittels Röntgenstrahlen,” in Kolloidchemie Ein Lehrbuch, Springer Berlin Heidelberg, 1912, pp. 387-409.

[131] S. Ibrahimkutty et al., "A hierarchical view on material formation during pulsed-laser synthesis of nanoparticles in liquid," Sci Rep, vol. 5, Nov. 2015.

[132] A. V. Kabashin and M. Meunier, "Synthesis of colloidal nanoparticles during femtosecond laser ablation of gold in water," Journal of Applied Physics, vol. 94, no. 12, p. 7941, 2003.

[133] M. Sivayoganathan, B. Tan, and K. Venkatakrishnan, "Effect of mega-hertz repetition rate on the agglomerated particle size of femtosecond synthesized nanostructures," Optical Materials Express, vol. 2, no. 8, pp. 987-995, 2012. 
[134] A. G. Rolo and M. I. Vasilevskiy, "Raman spectroscopy of optical phonons confined in semiconductor quantum dots and nanocrystals," J. Raman Spectrosc., vol. 38, no. 6, pp. 618633, Jun. 2007.

[135] V. Swamy, B. C. Muddle, and Q. Dai, "Size-dependent modifications of the Raman spectrum of rutile TiO2," Appl. Phys. Lett., vol. 89, no. 16, p. 163118, Oct. 2006.

[136] U. Balachandran and N. G. Eror, "Raman spectra of titanium dioxide," Journal of Solid State Chemistry, vol. 42, no. 3, pp. 276-282, May 1982.

[137] I. A. Alhomoudi and G. Newaz, "Residual stresses and Raman shift relation in anatase TiO2 thin film,” Thin Solid Films, vol. 517, no. 15, pp. 4372-4378, Jun. 2009.

[138] H. C. Choi, Y. M. Jung, and S. B. Kim, "Size effects in the Raman spectra of TiO2 nanoparticles," Vibrational Spectroscopy, vol. 37, no. 1, pp. 33-38, Jan. 2005.

[139] G. Gouadec and P. Colomban, "Raman Spectroscopy of nanomaterials: How spectra relate to disorder, particle size and mechanical properties," Progress in Crystal Growth and Characterization of Materials, vol. 53, no. 1, pp. 1-56, Mar. 2007.

[140] J. C. Parker and R. W. Siegel, "Calibration of the Raman spectrum to the oxygen stoichiometry of nanophase TiO2," Appl. Phys. Lett., vol. 57, no. 9, pp. 943-945, Aug. 1990.

[141] A. Li Bassi et al., "Raman spectroscopy characterization of titania nanoparticles produced by flame pyrolysis: The influence of size and stoichiometry," Journal of Applied Physics, vol. 98, no. 7, p. 074305, Oct. 2005.

[142] C. A. Melendres, A. Narayanasamy, V. A. Maroni, and R. W. Siegel, "Raman spectroscopy of nanophase $\mathrm{TiO}<$ span class='sub'>2</span $>$," Journal of Materials Research, vol. 4, no. 5, pp. 1246-1250, Oct. 1989. 
[143] J. C. Parker and R. W. Siegel, "Raman Microprobe Study of Nanophase TiO2 and Oxidation-Induced Spectral Changes," Journal of Materials Research, vol. 5, no. 6, pp. 1246-1252, 1990.

[144] G. Xing-Yuan, X. Da-Peng, D. Zhan-Hui, and S. Wen-Hui, "Preparation and Raman Spectrum of Rutile Single Crystals Using Floating Zone Method," Chinese Phys. Lett., vol. 23, no. 6, p. $1645,2006$.

[145] Y. Hara and M. Nicol, "Raman spectra and the structure of rutile at high pressures," phys. stat. sol. (b), vol. 94, no. 1, pp. 317-322, Jul. 1979.

[146] J.-Y. Eom, S.-J. Lim, S.-M. Lee, W.-H. Ryu, and H.-S. Kwon, "Black titanium oxide nanoarray electrodes for high rate Li-ion microbatteries," J. Mater. Chem. A, vol. 3, no. 21, pp. 11183-11188, May 2015.

[147] R. López and R. Gómez, "Band-gap energy estimation from diffuse reflectance measurements on sol-gel and commercial TiO2: a comparative study," J Sol-Gel Sci Technol, vol. 61, no. 1, pp. 1-7, Jan. 2012.

[148] B. H. Morgan, “A COMPILATION OF SELECTED DATA ON SOLAR RADIATION AT SEA LEVEL," 1967.

[149] M. Moskovits, "Surface-enhanced Raman spectroscopy: a brief retrospective," J. Raman Spectrosc., vol. 36, no. 6-7, pp. 485-496, Jun. 2005.

[150] D. Qi, L. Lu, L. Wang, and J. Zhang, "Improved SERS Sensitivity on Plasmon-Free TiO2 Photonic Microarray by Enhancing Light-Matter Coupling," J. Am. Chem. Soc., vol. 136, no. 28, pp. 9886-9889, Jul. 2014.

[151] S. Cong et al., "Noble metal-comparable SERS enhancement from semiconducting metal oxides by making oxygen vacancies," Nature Communications, vol. 6, p. 7800, Jul. 2015. 
[152] A. Nakajima, S. Koizumi, T. Watanabe, and K. Hashimoto, "Photoinduced Amphiphilic Surface on Polycrystalline Anatase TiO2 Thin Films," Langmuir, vol. 16, no. 17, pp. 7048 7050, Aug. 2000.

[153] S. Karuppuchamy and J. M. Jeong, "Super-hydrophilic amorphous titanium dioxide thin film deposited by cathodic electrodeposition," Materials Chemistry and Physics, vol. 93, no. 2-3, pp. 251-254, Oct. 2005.

[154] H. Y. Lee, Y. H. Park, and K. H. Ko, "Correlation between Surface Morphology and Hydrophilic/Hydrophobic Conversion of MOCVD-TiO2 Films," Langmuir, vol. 16, no. 18, pp. 7289-7293, Sep. 2000.

[155] T. Zubkov, D. Stahl, T. L. Thompson, D. Panayotov, O. Diwald, and J. T. Yates, "Ultraviolet Light-Induced Hydrophilicity Effect on TiO2(110)(1×1). Dominant Role of the Photooxidation of Adsorbed Hydrocarbons Causing Wetting by Water Droplets," J. Phys. Chem. B, vol. 109, no. 32, pp. 15454-15462, Aug. 2005.

[156] N. Sakai, A. Fujishima, T. Watanabe, and K. Hashimoto, "Quantitative Evaluation of the Photoinduced Hydrophilic Conversion Properties of TiO2 Thin Film Surfaces by the Reciprocal of Contact Angle," J. Phys. Chem. B, vol. 107, no. 4, pp. 1028-1035, Jan. 2003.

[157] K. Hashimoto, H. Irie, and A. Fujishima, "TiO2 Photocatalysis: A Historical Overview and Future Prospects," Jpn. J. Appl. Phys., vol. 44, no. 12R, p. 8269, Dec. 2005. 


\section{Glossary}

\section{Acronyms}

2D

3D

ASTM

CV

CVD

EDX

EF

fcc

FE-SEM

FFT

HDD

HR-TEM

LDD

LSO

LSPR

NIR

PDF

PVD

QD
Two-Dimensional

Three-Dimensional

American Society for Testing and Materials

Crystal Violet

Chemical Vapour Deposition

Energy Dispersive X-ray

Enhancement Factor

Face centred cubic

Field Emission Scanning Electron Microscope

Fast Fourier Transform

High Density Defect

High Resolution Transmission Electron Microscope

Low Density Defect

Laser Spot Overlap

Localised Surface Plasmon Resonance

Near-Infrared

Powder Diffraction File

Physical Vapour Deposition

Quantum Dot 


$\begin{array}{ll}\text { SATP } & \text { Standard Ambient Temperature and Pressure } \\ \text { SCFH } & \text { Standard cubic feet per hour } \\ \text { SEM } & \text { Scanning Electron Microscope } \\ \text { SERS } & \text { Surface Enhanced Raman Scattering } \\ \text { SPR } & \text { Surface Plasmon Resonance } \\ \text { TEM } & \text { Transmission Electron Microscope } \\ \text { USP } & \text { Ultrashort Pulse } \\ \text { UV } & \text { Ultraviolet } \\ \text { vis-NIR } & \text { Visible and NIR } \\ \text { XPS } & \text { X-ray Photoelectron Spectroscopy } \\ \text { XRD } & \text { X-ray Diffraction }\end{array}$

\section{Units}

$\begin{array}{ll}\text { \% } & \text { Percentage } \\ \circ & \text { Degree } \\ \boldsymbol{\mu l} & \text { Microliter } \\ \boldsymbol{\mu m} & \text { Micrometer } \\ \mathbf{A} & \text { Ampere } \\ \AA & \text { Angstrom } \\ \mathbf{a t m} & \text { Atmosphere } \\ \mathbf{C} & \text { Celsius } \\ \mathbf{c m} & \text { Centimeter } \\ \mathbf{e V} & \text { Electronvolt }\end{array}$




$\begin{array}{ll}\mathbf{f s} & \text { Femtosecond } \\ \mathbf{g} & \text { Gram } \\ \mathbf{J} & \text { Joule } \\ \mathbf{K} & \text { Kelvin } \\ \mathbf{k H z} & \text { Kilohertz } \\ \mathbf{M} & \text { Molar } \\ \mathbf{M H z} & \text { Megahertz } \\ \mathbf{m m} & \text { Millimeter } \\ \mathbf{m o l} & \text { Mole } \\ \mathbf{m s} & \text { Millisecond } \\ \mathbf{n m} & \text { Nanometer } \\ \mathbf{p s} & \text { Picosecond } \\ \mathbf{W} & \text { Watt }\end{array}$

\section{Nomenclature}

$\begin{array}{ll}\kappa_{\mathrm{s}} & \text { Shape factor } \\ f_{\mathrm{f}} & \text { Fill factor } \\ \boldsymbol{A}_{\text {sub }} & \text { Area of substrate } \\ \boldsymbol{A}_{\text {Raman }} & \text { Raman laser spot area } \\ \boldsymbol{C}_{\text {abs }} & \text { Absorption cross section } \\ \boldsymbol{C}_{\text {sca }} & \text { Scattering cross section } \\ \boldsymbol{E}_{\mathrm{g}} & \text { Band Gap } \\ \boldsymbol{I}_{\text {hdd }} & \text { CV dye Raman peak intensity on HDD-nano } \mathrm{TiO}_{\mathrm{x}}\end{array}$




\begin{tabular}{|c|c|}
\hline$I_{\text {sub }}$ & CV dye Raman peak intensity on Ti substrate \\
\hline$K_{\mathrm{m}}$ & Material constant \\
\hline$L_{f}$ & Laser Fluence \\
\hline$N_{\mathrm{A}}$ & Avagadro constant \\
\hline$N_{\text {bulk }}$ & $\begin{array}{l}\text { Number of molecules contributing to the Raman signal from the Ti } \\
\text { substrate }\end{array}$ \\
\hline$N_{\text {eff }}$ & Effective number of pulses \\
\hline$N_{\text {surf }}$ & $\begin{array}{l}\text { Number of molecules contributing to the Raman signal from the } \\
\text { HDD-nano } \mathrm{TiO}_{\mathrm{x}}\end{array}$ \\
\hline$T_{\mathrm{m}}$ & Maximum temperature \\
\hline$T_{\mathrm{n}}$ & Average surface temperature \\
\hline$V_{\text {Dye }}$ & Dye volume \\
\hline$V_{\text {core }}$ & Volume fraction of core nanosphere \\
\hline$V_{\text {islands }}$ & Volume fraction of plasmon metal islands \\
\hline$h_{\mathrm{d}}$ & Half of depth of field \\
\hline$n_{\mathrm{a}}$ & Atom number density \\
\hline$t_{\mathrm{p}}$ & Pulse duration \\
\hline$\alpha_{\mathrm{d}}$ & Polarizability of the metal nanoparticle \\
\hline$\alpha_{\mathrm{e}}$ & Extinction coefficient \\
\hline$\varepsilon_{\mathrm{m}}$ & Medium dielectric constant \\
\hline$\varepsilon_{\mathbf{o}}$ & Permittivity of free space \\
\hline$A$ & Laser absorption coefficient \\
\hline B & Absorption constant \\
\hline D & Deposition thickness \\
\hline$D$ & Laser spot diameter \\
\hline
\end{tabular}




\begin{tabular}{|c|c|}
\hline $\mathbf{D}_{\mathbf{p}}$ & Average crystallite size \\
\hline $\mathbf{E}$ & Photon energy \\
\hline $\boldsymbol{F}$ & Pulse frequency or repetition rate \\
\hline$I_{\mathrm{s}}$ & Sputtering current \\
\hline$K$ & Boltzmann constant \\
\hline $\mathbf{L}$ & Liquid \\
\hline$n$ & Number of laser pulses \\
\hline $\mathbf{P}$ & Laser power \\
\hline$r$ & Nuclei cluster radius \\
\hline $\boldsymbol{R}$ & Reflectance \\
\hline$S$ & Entropy \\
\hline$t$ & Deposition time \\
\hline $\mathbf{V}$ & Laser scanning speed \\
\hline $\mathbf{w}$ & Laser spot radius \\
\hline wt. \% & Weight percentage \\
\hline$\alpha$ & Constant ratio \\
\hline$\lambda$ & Wavelength \\
\hline$f$ & Focal length \\
\hline C & Molar Concentration \\
\hline$M$ & Molecular Weight \\
\hline$a$ & Thermal diffusion coefficient \\
\hline $\boldsymbol{c}$ & Speed of light \\
\hline $\boldsymbol{h}$ & Planks Constant \\
\hline$\varepsilon(\omega)$ & Complex dielectric function of metal particle \\
\hline $\boldsymbol{\beta}$ & Line broadening \\
\hline
\end{tabular}




$\begin{array}{ll}\boldsymbol{\varepsilon} & \text { Absorbance value from spectrum } \\ \boldsymbol{\theta} & \text { Bragg angle } \\ \boldsymbol{\kappa} & \text { Heat conduction coefficient } \\ \boldsymbol{\mu} & \text { Chemical potential } \\ \boldsymbol{\rho} & \text { Density } \\ \boldsymbol{\sigma} & \text { Surface tension } \\ \boldsymbol{v} & \text { Light frequency } \\ \boldsymbol{\omega} & \text { Frequency }\end{array}$

\section{Chemical Symbols}

(TiO.716)3.76

Ag

AgCl

Au

Au/Pd

CdS

CdSe

CdTe

$\mathrm{Ce}$

CIGS

$\mathrm{Cu}$

$\mathrm{CuO}$

GaAs
Non-stoichiometric Oxide of Titanium

Silver

Silver Chloride

Gold

Gold Palladium alloy

Cadmium Sulfide

Cadmium Selenide

Cadmium Telluride

Cerium

Copper Indium Gallium Selenide

Copper

Copper Oxide

Gallium Arsenide 


\begin{tabular}{ll} 
GaN & Gallium Nitride \\
$\mathbf{H}$ & Hydrogen \\
$\mathbf{N}$ & Nitrogen \\
$\mathbf{O}$ & Oxygen \\
$\mathbf{P b S}$ & Lead Sulfide \\
$\mathbf{P d}$ & Palladium \\
$\mathbf{R u}$ & Ruthenium \\
$\mathbf{S i}$ & Silicon \\
$\mathbf{S n}$ & Tin \\
$\mathbf{T i}$ & Titanium \\
$\mathrm{Ti}_{3} \mathrm{O}$ & Tri-Titanium Oxide \\
$\mathrm{Ti}_{3} \mathrm{O}_{5}$ & Tri-Titanium Pentaoxide \\
$\mathbf{T i O}_{2}$ & Titanium Dioxide \\
$\mathrm{TiO}_{\mathbf{x}}$ & Multiphase Oxide of Titanium \\
$\mathbf{Z n O}$ & Zinc Oxide \\
\hline
\end{tabular}

